
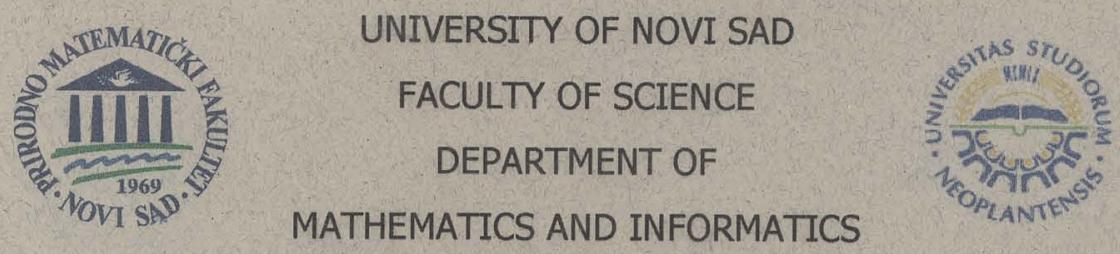

Aleksandar Pavlović, M. Sc.

\title{
SEQUENTIAL TOPOLOGIES \\ ON BOOLEAN ALGEBRAS
}

\author{
Ph. D. Thesis \\ Supervisor \\ Dr Miloš Kurilić
}

Novi Sad, 2008. 

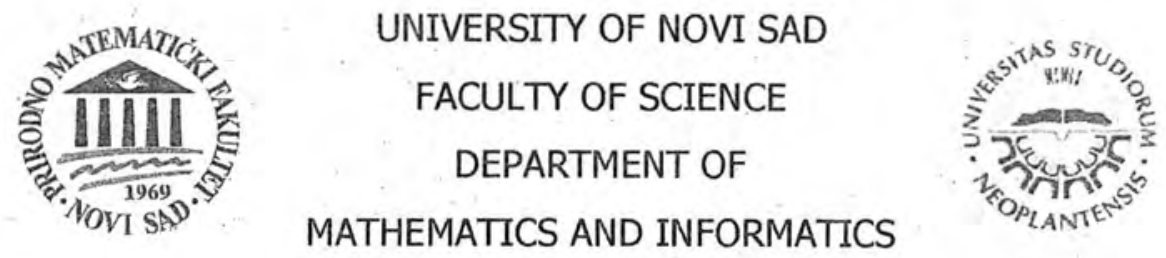

Aleksandar Pavlović, M. Sc.

\title{
SEQUENTIAL TOPOLOGIES ON BOOLEAN ALGEBRAS
}

\author{
Ph. D. Thesis \\ Supervisor \\ Dr Miloš Kurilić
}

Novi Sad, 2008. 


\begin{tabular}{|c|c|}
\hline UNIVERSITY OF NOVISAD \\
FACULTY OF SCIENCE \\
DEPARTMENT OF
\end{tabular}

Aleksandar Pavlović, M. Sc.

\section{SEQUENTIAL TOPOLOGIES ON BOOLEAN ALGEBRAS}

Ph.D. Thesis

Supervisor

Dr Miloš Kurilić́

Novi Sad, 2008. 
The mathematician does not study pure mathematics because it is useful; he studies it because he delights in it and he delights in it because it is beautiful.

Henri Poincaré 


\section{Contents}

Introduction iii

History ..................................... ii

The aim and structure of the thesis .............. vi

Acknowledgement .................... viil

I Preliminaries 1

1 Set Theory 3

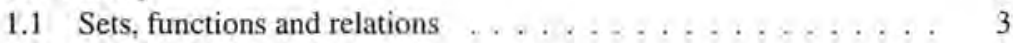

1.2 Partially ordered sets ................... . . . 4

1.3 Small cardinals .................... 5

2 General topology 7

2.1 Definitions and basic facts ... . . . . . . . . . . 7

2.2 Sequences in topological spaces ... . . . . . . . . . 11

2.3 Topologies generated by a priori limit operators . . . . . . . . . 14

2.3.1 Closures of an operator $\lambda$ under (LL), (L.2) and (L3) . . 18

2.3.2 The operators $u_{\lambda}$ and $\mathrm{cl}_{\omega_{1}} \ldots \ldots . . . . . . .22$

2.3.3 The equality $u^{2}=u$. Fréchet spaces ......... 24

3 Boolean algebras $\quad 27$

3.1 Definitions and basic facts .............. 27

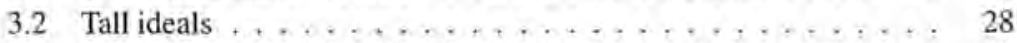

3.3 Sequences in Boolean algebras, lim sup and lim inf ...... 29

3.4 Measure and submeasure ................. 32

4 Forcing $\quad 35$

4.1 Generic extension . . . . . . . . . . . . . . . 35

4.2 Subsets of $\omega$ and sequences . . . . . . . . . . 37 
II Topologies on complete Boolean algebras

5 Sequential topology $\tau_{s} \quad 49$

5.1 Basic properties . . . . . . . . . . . . . . . . . . . 49

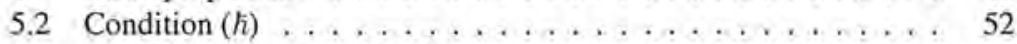

5.3 Condition $(h)$ and cellularity $\ldots \ldots \ldots \ldots \ldots \ldots . \ldots \ldots$

5.4 A posteriori convergence . . . . . . . . . . . 57

5.5 The sequential compactness $\ldots \ldots \ldots \ldots \ldots \ldots . \ldots 4$

6 The topology $\mathcal{O}^{\uparrow} \quad 67$

6.1 Closed sets and the closure operator ... . . . . . . 67

6.1 .1 Generating $\mathcal{O}^{\dagger}$ by an a priori limit operator ...... 67

6.1.2 A characterization of closed sets and some properties . . . 68

6.1 .3 The closure operator .............. 70

6.1 .4 Operator Dec . . . . . . . . . . . . . . . 73

6.1.5 Closed sets and their minimal elements . . . . . . . 74

6.2 The equality $u=u^{2} \ldots \ldots \ldots \ldots \ldots \ldots \ldots \ldots \ldots \ldots$

6.3 A posteriori limit in $\left\langle\mathbb{B}, \mathcal{O}^{\top}\right\rangle \ldots \ldots \ldots \ldots \ldots . \ldots \ldots$

6.4 A posteriori limit in case when $u=u^{2} \ldots \ldots \ldots \ldots \ldots . \ldots . \ldots$

6.5 A representation of closed sets in $\left\langle P(\omega), \mathcal{O}^{\top}\right\rangle \ldots \ldots \ldots \ldots . . . .99$

6.5.1 Subbase countably compact spaces .......... 100

6.5.2 Minimal elements of the closed set $-\operatorname{Min}(\mathbf{F}) \ldots \ldots . .102$

6.5 .3 Examples of closed sets . . . . . . . . . 106

$\begin{array}{lll}7 & \text { Other topologies } & 109\end{array}$

7.1 Topologies generated by other a priori limit operators . . . . . 109

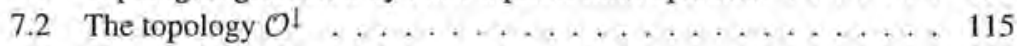

7.3 The topology $\mathcal{O}^{\star}$ and the connection with $\tau_{s} \ldots \ldots \ldots 116$

$\begin{array}{ll}\text { Questions } & 119\end{array}$

$\begin{array}{ll}\text { Bibliography } & 121\end{array}$

$\begin{array}{ll}\text { Prošireni apstrakt } & 129\end{array}$

$\begin{array}{ll}\text { Biografija } & 133\end{array}$ 


\section{Introduction}

\section{History}

In 1935, in the famous Scottish Coffee House in the city of Lwów (then Poland, now Ukraine) a group of mathematicians, led by Stefan Banach, begun their regular meetings, in which they discussed on mathematical problems. One morning they could not remember the solution of the problem solved last night and, therefore, they decided to write the problems into a notebook. This lasted until 1941. After the World War II Stanislaw Ulam, one of the regular participants, published the book titled "The Scottish Book" containing almost 200 problems from the notebook. The revised version of the book, edited by Daniel Mauldin, was published in 1981 [38].

On July 4th, 1937 John von Neumann wrote Problem 163: Is each ccc weakly distributive complete Boolean algebra a measure algebra? The prize for the solution was a bottle of whiskey of measure greater than zero. This problem is known as von Neumann's Problem.

In 1947 Dorothy Maharam [37] proved that each complete Boolean algebra which caries a continuous strictly positive submeasure (Maharam submeasure) is $\operatorname{ccc}$ and weakly distributive. Today a Boolean algebra carrying a Maharam submeasure is called a Maharam algebra. After that, von Neumann's Problem has been divided into two problems:

Problem 1: Is every Maharam algebra a measure algebra?

Problem 2: Is every ccc weakly distributive complete Boolean algebra a Maharam algebra?

The first problem is known as the Control Measure Problem. Considering this problem, D. Maharam has investigated the sequential topology $\tau_{s}$ on com-

plete Boolean algebras. She showed that the sequential topology $\tau_{s}$ on a Maharam algebra with a submeasure $m$ coincides with the topology induced by the metric $d$ defined by $d(a, b)=m(a \Delta b)$ and that a complete Boolean algebra $\mathbb{B}$ is a Maharam algebra iff the sequential topology $\tau_{s}$ on $\mathbb{B}$ is metrizable. Therefore, the Control Measure Problem can be reformulated: 
- Does the metrizability of the space $\left\langle\mathbb{B}, \tau_{3}\right\rangle$ imply $\mathbb{B}$ is a measure algebra?

In 1983 Kalton and Roberts [28] proved that every uniformly exhaustive submeasure on a Boolean algebra is equivalent to a finitely additive measure, where two submeasures $m$ and $\mu$ are equivalent iff $m\left(a_{n}\right) \rightarrow 0$ whenever $\mu\left(a_{n}\right) \rightarrow 0$ and vice versa. Since every Maharam submeasure is exhaustive, as a corollary of this theorem we have the following reformulation of the Control Measure Problem:

Is each exhaustive submeasure on a complete Boolean algebra uniformly exhaustive?

The statement that each exhaustive submeasure is uniformly exhaustive is equivalent to a $\Pi_{2}^{1}$ statement, which is absolute for inner models and for generic extensions. Therefore, if every Maharam algebra is a measure algebra in one model of $\mathrm{ZFC}$, then this holds in all its inner models and generic extensions. So, this problem can not be solved by forcing or by the tools for constructing inner models, developed by Gödel [24].

In January 2006 Michael Talagrand $[47,48]$ managed to construct an exhaustive, but not uniformly exhaustive submeasure on the Cantor algebra and, after 59 years, solved the Control Measure Problem. The answer is NO.

The nature of Problem 2 is completely different. In [37] D. Maharam showed that each Suslin algebra is a ccc weakly distributive complete Boolean algebra which does not carry a Maharam submeasure. So, the answer is NO. But the nonexistence of a Suslin algebra is consistent with ZFC. Therefore, Problem 2 was reformulated, and now it is known as the von Neumann-Maharam Problem:

Problem 2': Is it consistent with ZFC that every ccc weakly distributive complete Boolean algebra is a Maharam algebra?

This problem can be also reformulated using terms of the sequential topology $\tau_{s}$.

- Is the topology $\tau_{s}$ on a ccc weakly distributive complete Boolean algebra metrizable?

In 1998 Balcar, Główczyńsky and Jech [5] showed that $\tau_{s}$ is metrizable iff $\mathbb{B}$ is a cec algebra and $\left\langle\mathbb{R}, \tau_{s}\right\rangle$ is a Hausdorff space. In December 2003 Balcar, Jech and Pazák [6] proved that $\tau_{s}$ is metrizable iff $\mathbb{B}$ is weakly distributive and $\{0\}$ is $G_{\delta}$ set. In 2004 Stevo Todorčević [50] proved that $\mathbb{B}$ is a Maharam algebra iff it is weakly distributive and satisfies the $\sigma$-finite chain condition.

Finally, Balcar, Jech and Pazák [6] and, independently, Veličković [54] showed that under the P-ideal dichotomy (stated by Abraham and Todorčević [1] and extended by Todorčević [49]) every completé ccc weakly distributive Boolean algebra is a Maharam algebra. So, the answer to the von Neumann - Maharam problem is YES.

The Serbian mathematicians had a significant role in solving the von Neumann - Maharam Problem. We have already mentioned Stevo Todorčević and Boban 
Veličković. In 1935 Đuro Kurepa [34] defined a Suslin tree and proved that its existence is equivalent to the existence of a Suslin line. Completing a Suslin tree we obtain a Suslin algebra, a counterexample related to Problem 2.

The study of convergence structures is older than von Neumann's Problem. An a priori limit operator on the set $X$ is a mapping $\lambda: X^{\omega} \rightarrow P(X)$. In 1906 Fréchet made first steps in defining topological structures using a priori limit operators (see [21] and [22]). This was followed by Uryson [52]. Using an operator $\lambda$ such that $|\lambda(x)| \leq 1$, for each sequence $x$, they defined the sequential closure of a set $A$, and isolated necessary and sufficient conditions (L1)-(L4) for an a priori limit operator, such that the sequential closure coincides with the topological closure. In 1960, Kisyński [32] defined sequentially-closed sets and, in the case when $|\lambda(x)| \leq 1$, proved that an a priori limit operator satisfies (L1)-(L3) iff the family of closed sets coincides with the family of sequentially-closed sets.

In 1970, Antosik [2], considering the case when the limit of a sequence is not unique, gave a sufficient condition for an a priori limit operator to be topological. Having this result as a starting point, and motivated by problems of Mikusinski operator calculus, in the late 70's, a group of Polish mathematicians improved Antosik's results. Kamiński [29] simplified Antosik's result omitting one of the conditions. Ferens, Kamiński and Kliś [18] showed, by examples, that some sufficient conditions are not necessary. Kamiński in [30] (see also [31]) studied the relations between the properties of a priori limit operators. He presented sufficient and necessary conditions for an a priori limit operator to be topological, but these conditions were not operative. All of these results were presented on the Conference of Convergence, held in Szczyrk in October 1979.

Sequential and Fréchet spaces belonged to the folklore almost since the origin of general topology, but their extensive examination started Franklin in 1965 (see [19] and [20]).

The a priori limit operators are not strong enough for describing each topological structure. The first steps in generalization of sequences and their limits were made in 1915 by E.H. Moore (see [40] and [41]), He has considered nets, generalized sequences indexed by directed sets (partial orders in which each two elements have a common upper bound), In $1937 \mathrm{G}$. Birkhoff [9] applied nets in general topology. 


\section{The aim and structure of the thesis}

Since Von Neumann's Problem is related to a restricted class of c.B.a.'s (weakly distributive and $(c c)$, the sequential topology on other Boolean algebras is not widely investigated. Hence, the first aim of this thesis is to investigate other properties of the sequential topology which are relevant to a wider class of c.B.a.'s.

The sequential topology on c.B.a. is just one example of using an a priori limit operator in defining topological structures. Therefore, the second aim of the thesis is to obtain other topologies on c.B.a.'s, changing the initial a priori limit operator, and to explore the relations between the algebraic and topological properties of Boolean algebras.

The thesis is divided into two parts.

In the first part the known facts about the tools and structures we will be dealing with are collected.

Chapter 1 contains definitions related to sets, functions, partially ordered sets and small cardinals.

In Chapter 2 some basic facts concerning topological spaces and limit operators are given. Also a tool for constructing a topology on a set $X$ using an a priori limit operator $\lambda: X^{\omega} \rightarrow P(X)$ is developed. This idea is not a new one and can be found in Uryson's papers (see [52] and [16]). But our method does not demand any prerequisites to $\lambda$, which distinguish these two methods.

Chapter 3 gives some elementary information about Boolean algebras with the accent on the properties of algebraic convergence of sequences.

Chapter 4 is reserved for forcing. Some basic notions concerning forcing are presented and the effects of forcing on the set $P(\omega)$ are considered. Each sequence in a Boolean algebra determines a name for a subset of $\omega$. Each subset of $\omega$ determines a real. The Boolean values of some properties of reals are calculated and two Boolean values, $a_{x}$ and $b_{x}$, related to sequences are introduced. Also, the basic distributivity laws in Boolean algebra are presented.

In the second part the results concerning topologies on complete Boolean algebras are given.

Chapter 5 is reserved for the already mentioned sequential topology $\tau_{s}$ on complete Boolean algebras. In Section 5.1 the known results related to $\tau_{s}$ are presented. In Section 5.2 the class of limsup-stable algebras (algebras which satisfy condition $(\hbar)$ ) is isolated and in Section 5.3 its relations to other classes are investigated. Section 5.4 is reserved for new results about topological limit in Boolean algebras with the sequential topology. It is known that the algebraic convergence, as an a priori convergence does not coincide with the topological convergence (the a pos- 
teriori convergence). Here we give two characterizations of both convergences, one using tall ideals and the second one using forcing, Also, it is proved that a necessary condition for the a posteriori convergence is $a_{x}=b_{x}$, which is also a sufficient condition in the class of lim sup-stable algebras. In Section 5.5 the class of sequentially compact spaces with the sequential topology is characterized. It is proved that the space $\left(\mathbb{B}, \tau_{s}\right)$ is sequentially compact iff $\mathbb{B}$ is a limsup-stable algebra and forcing by $\mathbb{B}$ does not produce independent reals.

The main topic of Chapter 6 is investigating the topology obtained using an the a priori limit operator $\lambda_{\text {sup }}$, defined on a c.B.a. by $\lambda_{\text {sup }}(x)=\{\limsup x\}$. Closing the operator $\lambda_{\text {sup }}$ to fulfill (L2) is the first task in Section 6.1. Since, $\bar{\lambda}_{s u p}(x)=$ $(\lim \sup x) \uparrow$, the topology generated by $\lambda_{\text {sup }}$ is denoted by $\mathcal{O}^{\top}$. Properties of the operators $u$ and $\mathrm{cl}_{\omega_{1}}$ are investigated, closure of some specific sets has been determined, and, using a new set operator Dec, a closed set in the space $\left\langle\mathbb{B}, \mathcal{O}^{\dagger}\right\rangle$ is characterized as an upward closed $\omega_{1}$-closed set. In such way we have obtained a $T_{\mathbb{Q}}$, connected, compact space in which $a \vee x$ and $a \wedge x$ are continuous functions for each fixed $a$. In ccc c.B.a.'s a closed set is described by the family of its minimal elements. Some necessary conditions for a subset of $\mathbb{B}$ to be the set of minimal elements of a closed set are isolated.

Section 6.2 gives an answer to the question: which algebraic properties of a c,B.a. $\mathbb{B}$ ensures that the space $\left\langle\mathbb{B}, \mathcal{O}^{\dagger}\right\rangle$ is Fréchet? Since the property $u=u^{2}$ implies that the space $\left\langle\mathbb{B}, \mathcal{O}^{\top}\right\rangle$ is a Fréchet space, the Boolean algebras with this property are fully characterized. They are weakly distributive b-cc c.B.a.s.

In Sections 6.3 and 6.4 the properties of the a posteriori limit in the space $\left\langle\mathbb{B}, \mathcal{O}^{\dagger}\right\rangle$ are investigated, firstly in general case, and then on weakly distributive b-cc c.B.a.s.

Section 6.5 characterizes the set of minimal elements of a closed set in the algebra $P(\omega)$, using the notion of subbase countable compactness.

Chapter 7 describes the topologies obtained by some other a priori limit operators. Also, $\mathcal{O}$ !, the dual topology of $\mathcal{O}^{\top}$ is introduced. Using the join of these two topologies as a subbase of a topological space, the new topology, $\mathcal{O}^{*}$, is obtained. It is proved that the topological limits in $\mathcal{O}^{+}$and $\tau_{s}$ coincide in the class of lim sup-stable algebras and that these two topologies are equal on $P(\omega)$. 


\section{Acknowledgement}

Several years ago, then still unsolved the von Neuman-Maharmam problem has been presented to my supervisor Miloš Kurilić as the interesting combination of Set Theory and General Topology, Since Miloš Kurilić and I have tried to find a topic for my PhD thesis in which would be successfully applied everything learned during my PhD studies we have started to examine this topic. As one of the results of this joint work (among large amount of drunk coffees) is this thesis. His great knowledge, experience and devotion to work have been to me of great importance, and also an additional motivation. His help in preparation of the thesis has made it more readable and lucid. Therefore, I wish to thank him for this and for everything he learned me during these years (mad families are not mad at all).

I wish to thank all colleges and friends from the Department of Mathematics and Informatics (and all others) for pleasant, relaxed, but also working surrounding. Specially, I wish to thank members (formal and informal) of our project (Miloš Kurilić, Milan Grulović, Boris Šobot, Slavica Medić and Nada Perić) and members of our regular Monday seminar (they will be not mention by name, since there are so many of them) under supervision of Acad. Dr. Bogoljub Stanković. Also, I wish to thank professors Olga Hadžić, Ljiljana Gajić, Stevan Pilipović, Mirko Budinčević and Milan Grulović for all support and advices during my PhD studies, preparation of this thesis and my work as a teaching assistant.

I owe to my parents, Mira and Vlada, for embedding into me the love for Mathematics and for everything they gave during my life under their safety and after that.

I owe more than just a gratitude to my family, Jaca, Luka and Matija, for all love, support, and above all for their patience.

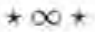

Pre par godina Milošu Kuriliću, mom mentoru, je predstavljen tada još nerešen von Neuman-Maharam problem kao interesantna veza teorije skupova i opšte topologije. S obzirom da smo Miloš Kurilić i ja tada tražili pogodnu temu za moj doktorat koja bi uspešno objedinila sve naučeno tokom mojih post-diplomskih studija, zajedno smo krenuli da je proučavamo. Kao jedan od rezultata tog zảjedničkog rada (pored gomile popijenih kafa) je i ova teza. Njegovo veliko znanje, iskustvo i predanost poslu su mi bili od velikog značaja, ali ujedno i dodatna motivacija. Njegova pomoć u sređivanju sadržaja i materijala je učinila ovu tezu preglednijom i čitljivijom. Stoga mu se ovom prilikom na svemu tome, kao i na svemu što me je ovih godina naučio, zahvaljujem.

Zahvaljujem se svim kolegama i prijateljima sa Departmana za matematiku 
i informatiku (a i šre) koji su doprineli prijatnoj, opuštenoj, ali nadasve radnoj atmosferi na poslu. Posebno bih izdvojio članove (formalne i neformalne) našeg projekta (Miloša Kurilića, Milana Grulovića, Borisa Šobota, Slavicu Medić i Nadu Perić), kao i članova redovnog seminara ponedeljkom (koje zbog brojnosti neću poimence nabrajati) pod rukovodstvom akad. dr Bogoljuba Stankovića. Takođe bih hteo da se zahvalim profesorima Olgi Hadžić, Ljiljani Gajić, Stevanu Pilipoviću, Mirku Budinčeviću i Milanu Gruloviću na svoj podršci i savetima koje sam od njih dobijao tokom post-diplomskih studija, tokom izrade ove teze, kao i tokom mog nastavnog rada kao asistenta.

Hvala mojim roditeljima, Miri i Vladi, za usađenu ljubav prema matematici, kao i za sve ostalo što su mi pružili tokom života pod njihovom zaštitom i nakon toga.

Mojoj porodici, Jaci, Luki i Matiji, dugujem više od zahvalnosti na svoj ljubavi, podršci, a pre svega strpljenju ("Tata će doktorikati").

Novi Sad, September 8, 2008

M.Sc. Aleksanđar Pavlović 



\section{Part I}

\section{Preliminaries}



The infinite!

No other question has ever moved so profoundly the spirit of man.

David Hilbert

\section{Chapter 1}

\section{Set Theory}

This chapter contains definitions related to sets, functions, partially ordered sets and small cardinals.

\subsection{Sets, functions and relations}

$\omega$ denotes the set of natural numbers. For a set $A$ and a cardinal $\kappa, P(A)$ denotes the power set of $A,[A]^{\kappa}$ the family of subsets of $A$ of cardinality $\kappa$, and $[A]^{<\kappa}$ the family of subsets of $A$ of cardinality less than $\kappa$. Therefore, $[\omega]^{\omega}$ is the family of all infinite subsets of $\omega$ and $|\omega|^{<\omega}$ is the family of all finite subsets of $\omega$.

For two sets, $A$ and $B, B^{A}$ denotes the family of all functions from $A$ into $B$, in particular, $A^{\omega}$ is the family of all functions from $\omega$ into $A$ (sequences in $A$ ). $A^{<\omega}=\bigcup_{n \in \omega} A^{n}$ is the family of finite approximations of sequences in $A$ and $\omega^{\dagger \omega}$ denotes the family of strictly increasing functions from $\omega$ into $\omega$. By $i d_{A}$ is denoted the identity mapping from the set $\mathrm{A}$ into itself defined by $i d_{A}(x)=x$. If $f: A \rightarrow B$ and if $X \subset A$ and $Y \subset B$, then $f[X]=\{f(x): x \in X\}$ and $f^{-1}[Y]=\{a \in A ; f(a) \in Y\}$.

For a set $A$, each subset $\varrho \subset A^{2}$ is a binary relation on $A$. Instead of $\langle a, b\rangle \in \varrho$, we often write $a \varrho b$. The relation $\varrho$ is

- reflexive iff $a \varrho a$, for each $a \in A$; 
- symmetric iff $a \varrho b$ implies $b \varrho a$, for each $a, b \in A$;

- antisymmetric iff $a \varrho b$ and $b \varrho a$ implies $a=b$, for each $a, b \in A$;

- transitive iff $a \varrho b$ and $b \varrho c$ implies $a \varrho c$, for each $a, b, c \in A$.

A reflexive, symmetric and transitive relation is called an equivalence relation. A reflexive, antisymmetric and transitive relation is called a partial ordering.

\subsection{Partially ordered sets}

A pair $\langle P, \leq\rangle$, where $\leq$ is a partial ordering on $P$ is a partially ordered set Let $\emptyset \neq X \subset P$. An element $a \in P$ is:

- an upper bound of $X$ iff $\forall x \in X x \leq a$;

- a lower bound of $X$ iff $\forall x \in X a \leq x$;

- the supremum of $X(\sup X)$ iff $a$ is an upper bound of $X$ and for each upper bound $b$ of $X$ there holds $a \leq b$;

- the infinum of $X(\inf X)$ iff $a$ is a lower bound of $X$ and for each lower bound $b$ of $X$ there holds $b \leq a$;

- the maximum of $X$ iff $a \in X$ and for each $x \in X$ there holds $x \leq a$;

- the minimum of $X$ iff $a \in X$ and for each $x \in X$ there holds $a \leq x$.

Let $\left\langle P_{1} \leq\right\rangle$ be a partially ordered set. A set $A \subset P$ is upward closed iff $b \geq$ $a \in A$ implies $b \in A$. A set $A \subset P$ is downward closed iff $b \leq a \in A$ implies $b \in A$. Downward closed sets are also called open sets. A set $D \subset P$ is dense in $P$ iff for each $x \in P$ there exists $y \in D$ such that $y \leq x$. Elements $x, y \in P$ are compatible iff there exists $z \in P$ such that $z \leq x$ and $z \leq y$. Otherwise, they are incompatible. Elements $x$ and $y$ are comparable iff $x \leq y$ or $y \leq x$.

A set $A \subset P$ is a chain iff each two elements of $A$ are comparable. A set $A \subset P$ is an antichain iff all elements of $A$ are pairwise incompatible. A partially ordered set $\langle P, \leq\rangle$ satisfies the countable chain condition (briefly $c c c$ ) iff each antichain in $P$ is countable.

Two partially ordered sets $\left\langle P_{1}, \leq_{1}\right\rangle$ and $\left\langle P_{2}, \leq_{2}\right\rangle$ are isomorphic iff there exists a bijection $f: P_{1} \rightarrow P_{2}$ such that $a \leq b \Leftrightarrow f(a) \leq f(b)$.

A partially ordered set $\langle P, \leq\rangle$ is linearly ordered iff each two elements of $P$ are comparable. $P$ is well-ordered iff each nonempty set $A \subset P$ has the minimum. A tree is a partially ordered set $\langle T, \leq\rangle$ such that for each $t \in T$, the set $\{s \in T$ : $s<t\}$ is well-ordered by the relation $\leq$. 


\subsection{Small cardinals}

According to the well known result of Cohen [14] it is consistent that the first uncountable cardinal $\aleph_{1}$ is less than the continuum $c$, the cardinality of the power set of $\omega$. The so called small cardinals express the size of subsets of $P(\omega)$ or $\omega^{i}$ and all of them are between $\aleph_{1}$ and $c$. Of course, assuming $\mathrm{CH}$, they are all equal to $\mathrm{c}$.

Sets $A$ and $B$ are almost disjoint iff the set $A \cap B$ is finite. $A \subset[\omega]^{\omega}$ is an almost disjoint family (briefly $a d f$ ) iff each two sets from $\mathcal{A}$ are almost disjoint. An adf $\mathcal{A}$ is a maximal almost disjoint family (briefly madf) iff for each $X \in[\omega]^{\omega}$ there exists $A \in \mathcal{A}$ such that the set $A \cap X$ is infinite, and the cardinal $a$ is defined by

$$
a=\min \left\{|\mathcal{A}|: \mathcal{A} \subset[\omega]^{\omega} \text { is an infinite madf }\right\} .
$$

An infinite set $S \subset \omega$ splits a set $A \subset \omega$ iff the sets $A \cap S$ and $A \backslash S$ are infinite. $\mathcal{S} \subset[\omega]^{\omega}$ is a spliting family iff for each infinite $A \subset \omega$ there exists $S \in \mathcal{S}$ such that $S$ splits $A$. The cardinal $s$ is defined by

$$
\mathfrak{s}=\min \left\{|S|: S \subset[\omega]^{\omega} \text { is a splitting family }\right\} .
$$

For sets $A, B \subset \omega$ let $A \subset^{*} B$ iff $A \backslash B$ is finite. Also $A \subsetneq^{*} B$ iff $A \subset^{*} B$ and $B \not \subset^{*} A$. An infinite set $Q$ is a pseudointersection of a family $\mathcal{P} \subset[\omega]^{\omega}$ iff $Q \subset^{*} P$ for each $P \in \mathcal{P}$. A family $\mathcal{P}$ has the strong finite intersection property (briefly sfip) iff each finite subfamily $\left\{P_{1}, P_{2}, \ldots, P_{n}\right\}$ of $\mathcal{P}$ has infinite intersection. A family $\mathcal{T} \subset[\omega]^{\omega}$ is a tower iff $\langle\mathcal{T}, \supset\rangle$ is a well-ordered set and $\mathcal{T}$ has no pseudointersection. The cardinals $p, t$ and $h$ are defined by

$$
\begin{aligned}
& p=\min \left\{|\mathcal{P}| ; \mathcal{P} \subset\left[\left.\omega\right|^{\omega} \text { has the sfip and has no pseudointersection }\right\}\right. \text {. } \\
& \mathrm{l}=\min \left\{|\mathcal{T}|: \mathcal{T} \subset[\omega]^{\omega} \text { is a tower }\right\} . \\
& \left.\zeta=\min \{|\mathcal{H}|: \mathcal{H} \text { is a family of open dense subsets of }\langle| \omega]^{\omega^{*}}, C^{*}\right\rangle \\
& \text { and } \bigcap \mathcal{H} \text { is not dense }\} \text {. }
\end{aligned}
$$

For functions $f, g \in \omega^{\omega}$ let $f \leq^{*} g$ iff there exists $k_{0} \in \omega$ such that $k \geq k_{0}$ implies $f(k) \leq g(k)$. B $\subset \omega^{\omega}$ is a bounded family iff there exists $g \in \omega^{\omega}$ such that $f \leq^{*} g$, for each $f \in \mathcal{B}$. D $\subset \omega^{\omega}$ is a dominating family iff for each $f \in \omega^{\omega}$ there exists $g \in \mathcal{D}$ such that $f \leq^{*} g$. The cardinals $b$ and $\mathfrak{o}$ are defined by

$$
\mathfrak{b}=\min \left\{|\mathcal{B}|: \mathcal{B} \subset \omega^{\omega} \text { is an unbounded family }\right\} \text {. }
$$




$$
\mathcal{\partial}=\min \left\{|\mathcal{D}|: \mathcal{D} \subset \omega^{\omega} \text { is a dominating family }\right\} .
$$

More information about the small cardinals can be found in [15] and [12].

The relations between small cardinals mentioned above are given in Figure 1.1. A line between $\kappa$ and $\lambda$, where $\kappa$ is under $\lambda$, means that $\kappa \leq \lambda$ is a theorem of ZFC.

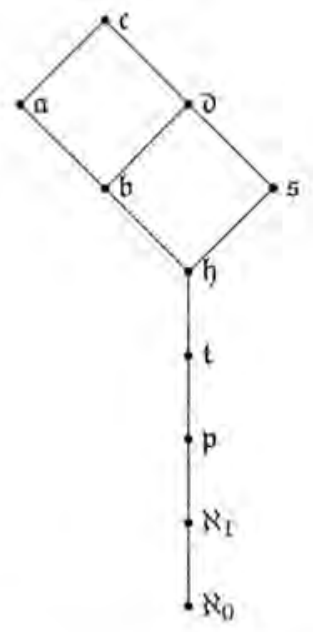

Figure 1.I: The ZFC inequalities between the small cardinals 
Pure mathematics is, in its way, the poetry of logical ideas.

Albert Einstein

\section{Chapter 2}

\section{General topology}

In this chapter some basic facts concerning topological spaces and limit operators are given. Also a tool for constructing a topology on a set $X$ using an a priori limit operator $\lambda: X^{\omega} \rightarrow P(X)$ is developed.

\subsection{Definitions and basic facts}

\section{Open and closed sets}

Let $X$ be a non-empty set. A family $\mathcal{O} \subset P(X)$ is a topology on $X$ iff

(O1) $\emptyset_{1} X \in \mathcal{O}$;

(O2) $O_{1}, O_{2} \in \mathcal{O} \Rightarrow O_{1} \cap O_{2} \in \mathcal{O}$;

(O3) $\mathcal{A} \subset \mathcal{O} \Rightarrow \cup \mathcal{A} \in \mathcal{O}$.

The elements of $\mathcal{O}$ are called open sets, and the pair $\langle X, \mathcal{O}\rangle$ is a topological space. A set $F \subset X$ is closed iff $X \backslash F$ is open. The family of closed sets is denoted by $\mathcal{F}$ and has the following properties:

(C1) $\emptyset, X \in \mathcal{F}$;

(C2) $F_{1}, F_{2} \in \mathcal{F} \Rightarrow F_{1} \cup F_{2} \in \mathcal{F}$;

(C3) $\emptyset \neq \mathcal{A} \subset \mathcal{F} \Rightarrow \cap \mathcal{A} \in \mathcal{F}$.

If an arbitrary family $\mathcal{F} \subset P(X)$ satisfies conditions $(\mathrm{C} 1)-(\mathrm{C} 3)$, then $\mathcal{O}=$ $\{X \backslash F: F \in \mathcal{F}\}$ is a topology on the set $X$. 
$A \subset X$ is a clopen set iff $A \in \mathcal{O} \cap \mathcal{F}$. A countable union of closed sets is called an $F_{\sigma}$-set. A countable intersection of open sets is called a $G_{\delta}$-set.

A point $x \in X$ is isolated in $\langle X, \mathcal{O}\rangle$ iff $\{x\} \in \mathcal{O}$. A space without isolated points is called perfect.

For two topologies $\mathcal{O}_{1}$ and $\mathcal{O}_{2}$ on a set $X$ such that $\mathcal{O}_{1} \subset \mathcal{O}_{2}$ we say that $\mathcal{O}_{2}$ is finer than $\mathcal{O}_{1}$, or that $\mathcal{O}_{1}$ is coarser than $\mathcal{O}_{2}$.

\section{Base and subbase}

If $\langle X, \mathcal{O}\rangle$ is a topological space, a family $\mathcal{B} \subset P(X)$ is a base for the topology $\mathcal{O}$ iff:

(BI) $B \subset \mathcal{O}$;

(B2) Each open set is the union of some subfamily of $\mathcal{B}$.

A family $\mathcal{P} \subset P(X)$ is a subbase for $\mathcal{O}$ iff

(PB1) $P \subset \mathcal{O}$;

(PB2) The family of finite intersections of members of $\mathcal{P}$ is a base for $\mathcal{O}$.

A family $\mathcal{B} \subset P(X)$ is called a base for some topology on the set $X$ iff $\left\{\cup \mathcal{B}_{1}\right.$; $\left.\mathcal{B}_{1} \subset \mathcal{B}\right\}$ is a topology on $X$. This holds iff

(BN1) $\cup B=X$;

(BN2) $\forall B_{1}, B_{2} \in \mathcal{B} \exists \mathcal{B}_{1} \subset \mathcal{B}\left(B_{1} \cap B_{2}=\bigcup \mathcal{B}_{1}\right)$.

A family $\mathcal{P} \subset P(X)$ is a subbase for some topology on $X$ iff $\left\{\cap \mathcal{P}_{1}: \mathcal{P}_{1} \in\right.$ $\left.[\mathcal{P}]^{<\omega} \backslash\{\emptyset\}\right\}$ is a base for some topology on $X$. This holds iff $\cup \mathcal{P}=X$.

A topological space $\langle X, \mathcal{O}\rangle$ is second-countable iff there exists a countable base for the topology $\mathcal{O}$.

\section{Neighborhoods}

In a topological space $\langle X, \mathcal{O}\rangle$ a set $U \subset X$ is a neighborhood of $x \in X$ iff $x \in U \in \mathcal{O}$. The family of all neighborhoods of $x$ is denoted by $\mathcal{U}(x)$.

A family $\mathcal{B}(x)$ is a neighborhood base at the point $x$ iff

(BOI) $\mathcal{B}(x) \subset \mathcal{U}(x)$;

(BO2) $\forall U \in \mathcal{U}(x) \exists B \in \mathcal{B}(x)(B \subset U)$.

A topological space $\langle X, \mathcal{O}\rangle$ is first-countable iff a countable neighborhood base exists at each point $x$. Each second-countable space is first-countable.

\section{Closure, derived set and separable spaces}

In a topological space $\langle X, \mathcal{O}\rangle$ a closure of $A \subset X$, denoted by $\bar{A}$, is the smallest closed set containing $A$. The closure operator on the space $\langle X, \mathcal{O}\rangle$ has the following properties: 


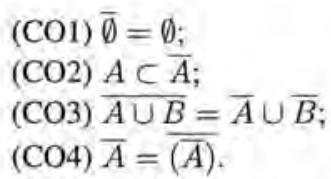

A point $x \in X$ is an accumulation point of $A$ iff $x \in \overline{A \backslash\{x\}}$. The set of all accumulation points of the set $A$ is called the derived set of $A$, denoted by $A^{d}$.

A set $D \subset X$ is dense in $X$ iff $\bar{D}=X$. If $\mathcal{B}$ is a base for the topology $\mathcal{O}$, then a set $D$ is dense in $X$ iff it meets each non-empty set from $\mathcal{B}$. A space $X$ is separable iff it contains a countable dense set. Each second-countable space is separable.

\section{Subspaces}

For a set $A \subset X$ the family $\mathcal{O}_{A}=\{O \cap A: O \in \mathcal{O}\}$ is the induced topology, and $\left\langle A, \mathcal{O}_{A}\right\rangle$ is a subspace of the space $\langle X, \mathcal{O}\rangle$. If $A \in \mathcal{O}$, then $A$ is an open subspace and if $A \in \mathcal{F}$, then $A$ is a closed subspace of $X$.

\section{Continuous mappings}

Let $\left\langle X_{1} \mathcal{O}_{X}\right\rangle$ and $\left\langle Y, \mathcal{O}_{Y}\right\rangle$ be topological spaces and let $x_{0} \in X$. A mapping $f: X \rightarrow Y$ is continuous at the point $x_{0}$ iff for each $V \in \mathcal{U}_{Y}\left(f\left(x_{0}\right)\right)$ there exists $U \in \mathcal{U}_{X}\left(x_{0}\right)$ such that $f[U] \subset V$. A mapping $f: X \rightarrow Y$ is continuous iff it is continuous at each point of $X$ iff for each $O \in \mathcal{O}_{Y}$ we have $f^{-1}[O] \in \mathcal{O}_{X}$. The mapping $f$ is open iff $O \in \mathcal{O}_{X}$ implies $f[O] \in \mathcal{O}_{Y}$ and $f$ is closed iff $F \in \mathcal{F}_{X}$ implies $f[F] \in \mathcal{F}_{Y}$. $f$ is a homeomorphism iff it is a continuous bijection and $f^{-1}$ is continuous. A continuous bijection $f$ is a homeomorphism iff it is open iff it is closed. Spaces $\left\langle X, \mathcal{O}_{X}\right\rangle$ and $\left\langle Y, \mathcal{O}_{Y}\right\rangle$ are homeomorphic, in notation $X \cong Y$, iff there exists a homeomorphism $f: X \rightarrow Y . f$ is an embedding iff its surjective restriction $\left.f\right|_{X}: X \rightarrow f[X]$ is a homeomorphism.

\section{Separation axioms}

A topological space $\langle X, \mathcal{O}\rangle$ is called

- a $T_{0}$-space iff $\forall x, y \in X \exists O \in \mathcal{O}(|O \cap\{x, y\}|=1)$;

- a $T_{1}$-space iff $\forall x, y \in X(x \neq y \Rightarrow \exists O \in \mathcal{O}(x \in O \not \nexists y))$;

- a $T_{2}$-space or a Hausdorff space iff $\forall x, y \in X\left(x \neq y \Rightarrow \exists O_{1}, O_{2} \in \mathcal{O}\left(x \in O_{1} \wedge y \in O_{2} \wedge O_{1} \cap O_{2}=\emptyset\right)\right)$; 
- a regular space iff $\forall F \in \mathcal{F} \forall x \in X \backslash F \exists O_{1}, O_{2} \in \mathcal{O}\left(x \in O_{1} \wedge F \subset O_{2} \wedge O_{1} \cap O_{2}=\emptyset\right)$, a regular $T_{1}$-space is called a $T_{3}$-space.

- a normal space iff $\forall F_{1}, F_{2} \in \mathcal{F}$ $\left(F_{1} \cap F_{1}=\emptyset \Rightarrow \exists O_{1}, O_{2} \in \mathcal{O}\left(F_{1} \subset O_{1} \wedge F_{2} \subset O_{2} \wedge O_{1} \cap O_{2}=\emptyset\right)\right)$, a normal $T_{1}$-space space is called a $T_{4}$-space.

A topological space is a $T_{1}$-space iff all singletons are closed.

It is well known that $T_{4} \Rightarrow T_{3} \Rightarrow T_{2} \Rightarrow T_{1} \Rightarrow T_{0}$.

\section{Covering properties}

A topological space $\langle X, \mathcal{O}\rangle$ is

- compact iff every open cover of the set $X$ has a finite subcover;

- sequentially compact iff each sequence in $X$ has a convergent subsequence;

- countably compact iff every infinite subset of $X$ has an accumulation point;

- a Lindelöf space iff it is a $T_{3}$-space and every open cover of the set $X$ has a countable subcover.

Each compact space is countably compact, and each sequentially compact space is countably compact.

\section{Connected spaces}

A topological space $\langle X, \mathcal{O}\rangle$ is connected iff it is not the union of two disjoint nonempty open sets. Otherwise, $X$ is disconnected. $X$ is zero-dimensional iff it is a $T_{1}$-space having a clopen base.

\section{Tychonoff product of spaces}

Let $\left\{\left\langle X_{i}, \mathcal{O}_{i}\right\rangle: i \in I\right\}$ be a family of topological spaces and for $j \in I$ let $p_{j}$ : $\prod_{i \in I} X_{i} \rightarrow X_{j}$ be the mappings defined by $p_{j}\left(\left\langle x_{i}: i \in I\right\rangle\right)=x_{j}$. Then the family $\bigcup_{i \in I}\left\{p_{i}^{-1}[O]: O \in \mathcal{O}_{i}\right\}$ is a subbase for the Tychonoff topology on the Cartesian product $\prod_{i \in I} X_{i}$. If all of the spaces $\left\langle X_{i}, O_{i}\right\rangle, i \in I$ are the same, then the product is denoted by $X^{I}$, where $X=X_{i}, i \in I$.

The Tychonoff product of compact Hausdorff spaces is a compact Hausdorff space.

By $2^{\kappa}$ is denoted the Cantor cube, the product of $\kappa$-many discrete two element spaces $\{0,1\} .2^{\omega}$ is known as the Cantor set. 


\section{Cardinal functions}

In this thesis we will consider the following cardinal functions:

- The weight of the space $\langle X, \mathcal{O}\rangle$ : $w(X)=\min \{|\mathcal{B}|: \mathcal{B}$ is a base for $\mathcal{O}\}+\aleph_{0}$.

- The character of the point $x$ in the space $\left\langle X_{i} \mathcal{O}\right\rangle$ : $\chi(x, X)=\min \{|\mathcal{B}(x)|: \mathcal{B}(x)$ is a neighborhood base at $x\}+\mathcal{N}_{0}$.

- The character of the space $\langle X, \mathcal{O}\rangle$ : $\chi(X)=\sup \{\chi(x, X): x \in X\}$.

- The pseudocharacter of a point $x$ in a $T_{1}$-space $\langle X, \mathcal{O}\rangle$ $\psi(x, X)=\min \{|\mathcal{U}|: \mathcal{U} \subset \mathcal{O} \wedge \cap \mathcal{U}=\{x\}\}+\aleph_{0}$.

- The pseudocharacter of a $T_{1}$-space $\langle X, \mathcal{O}\rangle$ $\psi(X)=\sup \{\psi(x, X) ; x \in X\}$.

- The density of the space $\langle X, \mathcal{O}\rangle$ : $d(X)=\min \{|D|: \bar{D}=X\}+\aleph_{0}$.

- The cellularity or the Suslin number of the space $\langle X, \mathcal{O}\rangle$ : $c(X)=\min \{\kappa:$ Each disjoint family of open sets has size $\leq \kappa\}+\aleph_{0}$.

- The Lindelö $f$ number of the space $\langle X, \mathcal{O}\rangle$ : $l(X)=\min \{\kappa:$ Each open cover has a subcover of size $\leq \kappa\}+\aleph_{0}$.

\subsection{Sequences in topological spaces}

\section{The limit operator}

A sequence $x: \omega \rightarrow X$ in the set $X$ will be denoted by $\left\langle x_{n}: n \in \omega\right\rangle$, or briefly by $\left\langle x_{n}\right\rangle$. A sequence $y$ is a subsequence of a sequence $x$ iff there exists $f \in \omega^{\dagger \omega}$ such that $y=x \circ f$.

Let $\langle X, \mathcal{O}\rangle$ be a topological space and $x=\left\langle x_{n}: n \in \omega\right\rangle$ a sequence in $X$. Then the sequence $x$ converges to a point $a$ of $X$ (or $a$ is a limit point of the sequence $x$ ) iff each neighborhood $U$ of the point $a$ contains all but finitely many members of the sequence $x$, i.e.

$$
x \rightarrow O a \stackrel{\text { def }}{\Longleftrightarrow} \forall U \in \mathcal{U}(a) \exists n_{0} \in \omega \forall n \geq n_{0} x_{n} \in U .
$$

The set of all limit points of the sequence $x$ will be denoted by limo $x$, or briefly by $\lim x$ when it is clear in which space we are working, and called the limit of 
the sequence $x$. In this way the operator limo : $X^{\omega} \rightarrow P(X)$ is determined and we call it the limit operator on the topological space $\langle X, \mathcal{O}\rangle$.

If every sequence in a topological space $\langle X, \mathcal{O}\rangle$ has at most one limit point, instead of $\lim x=\{a\}$, we will write $\lim x=a$.

Here are some elementary properties of the limit operator.

Fact 2.2.1 Let $x$ be a sequence in a topological space $\langle X, \mathcal{O}\rangle$. Then

(a)

$$
\begin{aligned}
\lim x & =\bigcap_{\left\{F \in \mathcal{F}: x_{n} \in F \text { for infinitely many } n \in \omega\right\}} \\
& =\bigcap_{A \in[\omega] \omega} \overline{\left\{x_{n}: n \in A\right\}} \\
& =\bigcap_{f \in \omega \uparrow \omega} \overline{\left\{x_{f(n)}: n \in \omega\right\}}
\end{aligned}
$$

(b) $\lim x$ is a closed set.

(c) $\left\{x_{n}: n \in \omega\right\} \cup \lim x \subset \overline{\left\{x_{n}: n \in \omega\right\}}$, but the reversed inclusion is not true in general.

(d) For each $m \in \omega$ there holds $\lim \left\langle x_{n}: n \in \omega\right\rangle=\lim \left\langle x_{m+n}: n \in \omega\right\rangle$.

Proof. (a) Let us prove that

$$
\lim x=\bigcap\left\{F \in \mathcal{F}: x_{n} \in F \text { for infinitely many } n \in \omega\right\} .
$$

(C) Let $a \in \lim x$ and let $F \in \mathcal{F}$ where $x_{n} \in F$ for infinitely many $n \in \omega$. The set $X \backslash F$ is an open set which does not contain all but finitely many members of the sequence $x$. Hence $a \notin X \backslash F$, that is $A \in F$.

(つ) Let $a \in A=\bigcap\left\{F \in \mathcal{F}: x_{n} \in F\right.$ for infinitely many $\left.n \in \omega\right\}$. Then $A$ is a closed set. Let us suppose that $a \notin \lim x$. Then there exists an open set $U$ containing $a$ such that $X \backslash U$ contains infinitely many members of $x$, so $a \in X \backslash U$.

To prove the second equality it is sufficient to prove that for each $F \in \mathcal{F}$ such that $x_{n} \in F$ for infinitely many $n \in \omega$ there exists $A \in[\omega]^{\omega}$ such that

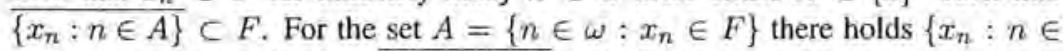
$A\} \subset F$, which implies that $\overline{\left\{x_{n}: n \in A\right\}} \subset F$. The last equality is obvious.

(b) is a direct consequence of (a).

(c) The inclusion follows directly from (a). Let $\mathbb{R}$ be the real line with the standard topology and let $x=\left\langle q_{n}: n \in \omega\right\rangle$ be an enumeration of the set of all rational numbers. Then $\lim x=\emptyset$, but $\overline{\left\{q_{n}: n \in \omega\right\}}=\mathbb{R}$.

(d) Follows directly from the first equality of (a).

Fact 2.2.2 Let $\langle X, \mathcal{O}\rangle$ be a topological space. Then $(\mathrm{a}) \Rightarrow(\mathrm{b}) \Rightarrow$ (c) where:

(a) $\langle X, \mathcal{O}\rangle$ is a Hausdorff space; 
(b) For each sequence $x$ in $X,|\lim x| \leq 1$;

(c) $\langle X, \mathcal{O}\rangle$ is a $T_{1}$-space.

Fact 2.2.3 For two topologies $\mathcal{O}_{1}$ and $\mathcal{O}_{2}$ on a non-empty set $X$ such that $\mathcal{O}_{1} \subset$ $\mathcal{O}_{2}$ we have that $\lim _{\mathcal{O}_{2}}(x) \subset \lim _{\mathcal{O}_{1}}(x)$, for each $x \in X^{\omega}$.

\section{Sequential and Fréchet spaces}

Definition 2.2.4 A topological space $\langle X, \mathcal{O}\rangle$ is

- sequential iff a set $A \subset X$ is closed whenever $\lim x \subset A$, for each sequence $x$ in $A$;

- a Fréchet space iff for every set $A \subset X$ we have $\bar{A}=\{a \in X: \exists x \in$ $\left.A^{\omega} a \in \lim x\right\}$.

\section{Fact 2.2.5}

(a) Each Fréchet space is sequential.

(b) A topological space $\langle X, \mathcal{O}\rangle$ is a Fréchet space iff for each set $A \subset X$ and each $b \in \bar{A} \backslash A$ there exists a sequence $x$ in $A$ such that $b \in \lim _{\mathcal{O}} x$.

(c) $[16,2.3 . K]$ The product of two Fréchet spaces must not be a sequential space.

Fact 2.2.6 Let $\left\langle X, \mathcal{O}_{X}\right\rangle$ and $\left\langle Y, \mathcal{O}_{Y}\right\rangle$ be topological spaces and $f: X \rightarrow Y$. Then

(a) [16, Prop. 1.6.6.] $f$ is continuous $\Rightarrow \forall x \in X^{\omega} f\left[\lim \left\langle x_{n}\right\rangle\right] \subset \lim \left\langle f\left(x_{n}\right)\right\rangle$.

(b) [16, Prop. 1.6.15.] If $X$ is a sequential space then " $\Leftrightarrow$ " holds in (a).

\section{Convergence structures, $\mathcal{S}^{*}$ and $\mathcal{L}^{*}$-spaces}

If $\langle X, \mathcal{O}\rangle$ is a topological space, then the pair $\langle X$, lim $)$ satisfies conditions (LI)(L3) from the definition of $\mathcal{L}^{*}$-spaces in the sense od Fréchet and Urysohn (see $[16,1.7 .18])$, except the uniqueness of the limit point. Precisely, there holds

Theorem 2.2.7 Let $\langle X, \mathcal{O}\rangle$ be a topological space and let $\lambda=\lim _{\mathcal{O}}: X^{\omega} \rightarrow$ $P(X)$ be the corresponding limit operator. Then

(L1) Each element $a$ of $X$ is a limit point of the constant sequence $(a: n \in \omega)$, i.e.

$$
\forall a \in X a \in \lambda(\langle a\rangle) .
$$

(L2) Each limit point of a sequence $x$ is also a limit point of each subsequence of $x$, i.e.

$$
\forall x \in X^{\omega} \forall f \in \omega^{\top \omega} \lambda(x) \subset \lambda(x \circ f) .
$$


(L3) If each subsequence of a sequence $x$ has a subsequence which converges to the point $a$, then the sequence $x$ also converges to $a$, i.e.

$$
\forall x \in X^{\omega}\left(\forall f \in \omega^{\uparrow \omega} \exists g \in \omega^{\dagger \omega} a \in \lambda(x \circ f \circ g) \Rightarrow a \in \lambda(x)\right) .
$$

In Fréchet spaces, the limit operator fulfils the additional condition from the definition of $\mathcal{S}^{*}$-spaces (see $[16,1.7 .18(\mathrm{~b})]$ )

Theorem 2.2.8 Let $\langle X, \mathcal{O}\rangle$ be a Fréchet space and let $\lambda=\lim _{\mathcal{O}}: X^{\omega} \rightarrow P(X)$ be the corresponding limit operator. Then

(L4) If $a \in \lambda\left(\left\langle x^{n}: n \in \omega\right\rangle\right)$ and if $x^{n} \in \lambda\left(\left\langle x_{i}^{n}: i \in \omega\right\rangle\right)$ for each $n \in \omega$, then there exists a sequence $x$ in $\left\{x_{i}^{n}: n, i \in \omega\right\}$ such that $a \in \lambda(x)$.

\subsection{Topologies generated by a priori limit operators}

An arbitrary a priori limit operator, the mapping $\lambda: X^{\omega} \rightarrow P(X)$ must not fulfil conditions (L1)-(L3). Therefore, it is possible that there does not exist a topology $\mathcal{O}$ on the set $X$ such that $\lambda=\lim _{\mathcal{O}}$. Since for the anti-discrete topology $\mathcal{O}_{a d}$ on $X$, we have $\lambda(x) \subset \lim _{\mathcal{O}_{a d}}(x)=X$, for each $x \in X^{\omega}$, the family $\Omega_{\lambda}$ of topologies $\mathcal{O}$ on the set $X$ satisfying

$$
\forall x \in X^{\omega} \lambda(x) \subset \lim _{\mathcal{O}}(x)
$$

where limo is the limit operator in the space $\langle X, \mathcal{O}\rangle$, is not empty. By Fact 2.2.3 finer topology produces smaller set of limit points. Therefore, it is natural to ask whether there is the maximal topology satisfying (2.2). For that purpose, on the set of all a priori limit operators on a set $X$ we define the order $\leq$ by

$$
\lambda_{1} \leq \lambda_{2} \Leftrightarrow \forall x \in X^{\omega} \lambda_{1}(x) \subset \lambda_{2}(x) .
$$

Theorem 2.3.1 Let $\lambda$ be an a priori limit operator defined on a non-empty set $X$. Let $\Omega_{\lambda}$ be a family of topologies $\mathcal{O}$ on the set $X$ satisfying (2.2). Then

(a) There exists a maximal topology $\mathcal{O}_{\lambda}$ in the family $\Omega_{\lambda}$;

(b) $\mathcal{O}_{\text {limo }_{\mathcal{O}_{\lambda}}}=\mathcal{O}_{\lambda}$, where $\mathcal{O}_{\text {lim }_{O_{\lambda}}}$ is the maximum of $\Omega_{\text {limo }}$;

(c) $\lim _{\mathcal{O}_{\lambda}}$ is the smallest limit operator greater than or equal to $\lambda$.

Proof. (a) The family $\Omega_{\lambda}$ is not empty, since the anti-discrete topology fulfils condition (2.2). The set $\bigcup \Omega_{\lambda}$ is a subbase for a topology on the set $X$ denoted by $\mathcal{O}_{\lambda}$. Obviously, for each $\mathcal{O} \in \Omega_{\lambda}$, we have $\mathcal{O} \subset \mathcal{O}_{\lambda}$. Let us prove that $\mathcal{O}_{\lambda}$ satisfies condition (2.2). Let $x$ be a sequence in $X$ and let $a \in \lambda(x)$, For each $O \in \mathcal{O}_{\lambda}$ such that $a \in O$ there exist topologies $\mathcal{O}_{i} \in \Omega_{\lambda}$ and sets $O_{i} \in \mathcal{O}_{i}, i=1,2, \ldots, k$ 
such that $a \in \bigcap_{i=1}^{k} O_{i} \subset O$. So, for each $i \leq k, a \in O_{i}$. Since $\mathcal{O}_{i} \in \Omega_{\lambda}$, we have $a \in \lim _{\mathcal{O}_{i}}(x)$ so there exists $n_{0}^{i}$ such that for all $n \geq n_{0}^{i}$ there holds $x_{n} \in O_{i}$. Therefore, for each $n \geq \max \left\{n_{0}^{i}: i \leq k\right\}$ we have that $x_{n} \in O$, which implies that $a \in \lim _{\mathcal{O}_{\lambda}}(x)$. So, $\mathcal{O}_{\lambda} \in \Omega_{\lambda}$.

(b) According to (a), for each topology $\mathcal{O}$ on the set $X$ satisfying $\lim _{\mathcal{O}_{\lambda}} \leq$ limo there holds $\mathcal{O} \subset \mathcal{O}_{\lim _{\mathcal{O}_{\lambda}}}$ so, for $\mathcal{O}=\mathcal{O}_{\lambda}$ we obtain $\mathcal{O}_{\lambda} \subset \mathcal{O}_{\text {lim }_{\mathcal{O}_{\lambda}}}$. On the other hand, since $\lambda \leq \lim _{\mathcal{O}_{\lambda}}$, we have $\Omega_{\text {lim } \mathcal{O}_{\lambda}} \subset \Omega_{\lambda}$ and since $\mathcal{O}_{\text {lim }_{\mathcal{O}_{\lambda}}} \in \Omega_{\text {lim }}$,

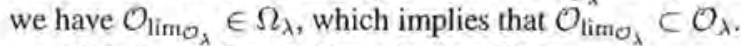

(c) Follows directly from the Fact 2,2.3.

The limit operator $\lim _{\mathcal{O}_{\lambda}}$ obtained in the previous lemma will be called the $a$ posteriori limit operator and the corresponding convergence will be called the $a$ posteriori convergence.

Lemma 2.3.2 If $\lambda_{1}$ and $\lambda_{2}$ are two a priori limit operators on $X$ such that $\lambda_{1} \leq \lambda_{2}$, then $\mathcal{O}_{\lambda_{2}} \subset \mathcal{O}_{\lambda_{1}}$.

Proof. Since $\lambda_{1} \leq \lambda_{2} \leq \lim _{\mathcal{O}_{2}}$ we have $\mathcal{O}_{\lambda_{2}} \in \Omega_{\lambda_{1}}=\left\{\mathcal{O}: \lambda_{1} \leq \lim _{\mathcal{O}}\right\}$, which, by the maximality of $\mathcal{O}_{\lambda_{1}}$, implies that $\mathcal{O}_{\lambda_{2}} \subset \mathcal{O}_{\lambda_{1}}$.

For an a priori limit operator $\lambda$, we can describe the topology $\mathcal{O}_{\lambda}$ in another way.

Theorem 2.3.3 (see [2]) If $\lambda: X^{\omega} \rightarrow P(X)$ is an a priori limit operator, then $\mathcal{O}_{\lambda}=\mathcal{T}_{\lambda}$ where

$$
\mathcal{T}_{\lambda}=\left\{O \subset X: \forall x \in X^{\omega}\left(O \cap \lambda(x) \neq \emptyset \Rightarrow \exists n_{0} \in \omega \forall n \geq n_{0} x_{n} \in O\right)\right\} .
$$

Proof. First let us prove that $\mathcal{T}_{\lambda}$ is a topology on the set $X$.

(O1) $\emptyset$ and $X$ are evidently in $\mathcal{T}_{\lambda}$.

(O2) Let $O_{1}, O_{2} \in \mathcal{T}_{\lambda}$, and let $x$ be a sequence in $X$. If $\left(O_{1} \cap O_{2}\right) \cap \lambda(x) \neq \emptyset$, then there exist $n_{0}^{1}$ and $n_{0}^{2}$ such that for all $n \geq n_{0}^{1}$ we have $x_{n} \in O_{1}$, and for all $n \geq n_{0}^{2}$ we have $x_{n} \in O_{2}$. Therefore, for $n_{0}=\max \left\{n_{0}^{1}, n_{0}^{2}\right\}$ and each $n \geq n_{0}$ we have that $x_{n} \in O_{1} \cap O_{2}$, which proves that $O_{1} \cap O_{2} \in \mathcal{T}_{\lambda}$.

(O3) Let $O_{i} \in \mathcal{T}_{\lambda}, i \in \mathcal{I}$. If $\bigcup_{i \in \mathcal{I}} O_{i} \cap \lambda(x) \neq \emptyset$, then there exists $i_{0}$ such that $O_{i_{0}} \cap \lambda(x) \neq \emptyset$. Therefore, there exists $n_{0}^{i_{0}}$ such that for all $n \geq n_{0}^{i_{0}}$ we have that $x_{n} \in O_{i_{0}} \subset \bigcup_{i \in \mathcal{I}} O_{i}$, which implies that $\bigcup_{i \in \mathcal{I}} O_{i} \in \mathcal{T}_{\lambda}$.

Now, let us prove that $\mathcal{T}_{\lambda}$ fulfils condition (2.2). Let $x$ be a sequence in $X$, $a \in \lambda(x)$ and $O$ an arbitrary neighborhood of the element $a$ in the topology $\mathcal{T}_{\lambda}$. Since $O \cap \lambda(x) \neq \emptyset$, by the definition of $\mathcal{T}_{\lambda}$, there exists an $n_{0}$ such that $x_{n} \in O$, for $n \geq n_{0}$. So, $a \in \lim _{\tau_{\lambda}}(x)$. Therefore, the topology $\mathcal{T}_{\lambda}$ satisfies (2.2), which implies that $\mathcal{T}_{\lambda} \subset \mathcal{O}_{\lambda}$. 
Let us prove that each $O \in \mathcal{O}_{\lambda}$ is a member of $\mathcal{T}_{\lambda}$. Let $x \in X^{\omega}$ such that $O \cap \lambda(x) \neq \emptyset$. By (2.2), for an $a \in O \cap \lambda(x)$ we have $a \in \lim _{\mathcal{O}_{\lambda}}(x)$. Therefore, there exists $n_{0}$ such that $x_{n} \in O$ for each $n \geq n_{0}$, which completes the proof.

In the sequel we show that, for an a priori limit operator $\lambda$ satisfying conditions (L1) and (L2), the topology $\mathcal{O}_{\lambda}$ can be described in one more way - by an explicit definition of closed sets.

Theorem 2.3.4 (see [31]) Let $\lambda$ be an a priori limit operator on a set $X$ satisfying (L1) and (L2) and let

$$
\mathcal{F}_{\lambda}^{\prime}=\left\{F \subset X: \forall x \in F^{\omega} \lambda(x) \subset F\right\} .
$$

(a) The family $\mathcal{F}_{\lambda}^{\prime}$ satisfies the axioms (C1)-(C3) for closed sets;

(b) $\mathcal{O}_{\lambda}=\left\{X \backslash \hat{F}: F \in \mathcal{F}_{\lambda}^{\prime}\right\}$.

Proof. (a) (C1) The sets $\emptyset$ and $X$ are obviously in $\mathcal{F}_{\lambda}^{\prime}$.

(C2) Let $A, B \in \mathcal{F}_{\lambda}^{\prime}$. Let us prove that for each $x=\left\langle x_{n}: n \in \omega\right\rangle \in(A \cup B)^{\omega}$ we have $\lambda(x) \subset A \cup B$. Without loss of generality, we can suppose that for each $n$ there exists $m \geq n$ such that $x_{m} \in A$. Therefore, there exists $y \prec x$ such that $y \in A^{\omega}$. Since $\lambda$ satisfies (L2), and since $\lambda(y) \subset A$, we have $\lambda(x) \subset \lambda(y) \subset A \subset$ $A \cup B$.

(C3) Let $A_{i}, i \in I$, be a family of sets belonging to $\mathcal{F}_{\lambda}^{\prime}$ and let $x$ be a sequence in $\bigcap_{i \in I} A_{i}$. Then $\lambda(x) \subset A_{i}$, for each $i \in I$, which implies $\lambda(x) \subset \bigcap_{i \in I} A_{i}$.

(b) Let $\mathcal{O}_{\lambda}^{\prime}=\left\{X \backslash F: F \in \mathcal{F}_{\lambda}^{\prime}\right\}$. Let us prove that $\mathcal{O}_{\lambda}^{\prime}=\mathcal{O}_{\lambda}$

(c) We check that $\mathcal{O}_{\lambda}^{\prime}$ satisfies (2.2). Let $x=\left\langle x_{n}: n \in \omega\right\rangle$ be a sequence in $X$ and $a \in \lambda(x)$. Suppose that $a \notin \lim _{\mathcal{O}_{x}^{\prime}} x$. Then there exists a neighborhood $U$ of the point $a$ which does not contain infinitely many members of the sequence $x$. Therefore, there exists a subsequence $y=\left\langle x_{n_{k}}: k \in \omega\right\rangle \prec x$ such $x_{n_{k}} \notin U$, $k \in \omega$. Since $\lambda$ fulfils (L2), we have that $a \in \lambda(y)$, and, according to (2.3), $a \in \overline{\left\{x_{n_{k}}: k \in \omega\right\}}$, but also $\left\{x_{n_{k}}: k \in \omega\right\} \subset X \backslash U$, which implies that $a \in X \backslash U$. A contradiction. So, the topology $\mathcal{O}_{\lambda}^{\prime}$ satisfies condition (2.2), which implies that $\mathcal{O}_{\lambda}^{\prime} \subset \mathcal{O}_{\lambda}$.

(כ) Suppose that there exists a closed set $F$ in the space $\left\langle X, \mathcal{O}_{\lambda}\right\rangle$ which is not closed in the space $\left\langle X, \mathcal{O}_{\lambda}^{\prime}\right\rangle$. Then there exists an element $a \in X \backslash F$ and a sequence $x$ in $F$ such that $a \in \lambda(x)$, and therefore $a \in \lim _{\mathcal{O}_{\lambda}} x$. The set $X \backslash F$ is in $\mathcal{O}_{\lambda}$ and contains $a$, but does not contain any member of the sequence $x$. A contradiction.

For an a priori limit operator $\lambda: X^{\omega} \rightarrow P(X)$ we have determined the maximal topology $\mathcal{O}_{\lambda}$ on the set $X$ satisfying (2.2). Therefore, the a posteriori convergence $\lim _{\mathcal{O}_{\lambda}}$ in the space $\left\langle X, \mathcal{O}_{\lambda}\right\rangle$ is determined by the a priori limit operator 
$\lambda$. The first question is: When the a priori and the a posteriori limit operator coincide? Obviously, by Theorem 2.2.7, (L1)-(L3) are necessary conditions for the a priori operator. Therefore, we can ask: Are conditions (L1)-(L3) sufficient for the equality of these two operators? According to [16, 1.7.19] (see also [32]) and Theorem 2.3.4, if $|\lambda(x)| \leq 1$ for each sequence $x$ in $X$ then the answer is YES. The following example will show that this is not true in general.

Example 2.3.5 The operator $\lambda: X^{\omega} \rightarrow P(X)$ satisfies (L1)-(L3), but the convergence a priori and a posteriori are not equal.

Let $X=\{1,2,3\}$. For a sequence $x=\left\langle x_{n}: n \in \omega\right\rangle \in X^{\omega}$ let us define $\operatorname{ran}_{\infty}(x)$ by

$$
\operatorname{ran}_{\infty}(x)=\left\{a \in X:\left|\left\{n: x_{n}=a\right\}\right|=\aleph_{0}\right\} .
$$

Let $\lambda: X^{\omega} \rightarrow P(X)$ be defined by

$$
\lambda(x)=\left\{\begin{array}{cl}
\{1,2\}, & \text { if } \operatorname{ran}_{\infty}(x)=\{1\}, \\
\{2,3\}, & \text { if } \operatorname{ran}_{\infty}(x)=\{2\}, \\
\{3\}, & \text { if } \operatorname{ran}_{\infty}(x)=\{3\}, \\
\{2\}, & \text { if } \operatorname{ran}_{\infty}(x)=\{1,2\}, \\
\emptyset, & \text { if } \operatorname{ran}_{\infty}(x)=\{1,3\}, \\
\{3\}, & \text { if } \operatorname{ran}_{\infty}(x)=\{2,3\}, \\
\emptyset, & \text { if } \operatorname{ran}_{\infty}(x)=\{1,2,3\} .
\end{array}\right.
$$

Since for each $a \in\{1,2,3\}$ we have $a \in \lambda(\langle a\rangle)$, the operator $\lambda$ satisfies (L1).

Claim 1. The operator $\lambda$ satisfies (L2).

Proof of Claim 1. Let $x$ be a sequence in $X$ and $y$ a subsequence of $x$. We will prove that $\lambda(x) \subset \lambda(y)$.

- If $\left|\operatorname{ran}_{\infty}(x)\right|=1$, then also, $\left|\operatorname{ran}_{\infty}(y)\right|=1$ and $\operatorname{ran}_{\infty}(x)=\operatorname{ran}_{\infty}(y)$. which implies that $\lambda(x)=\lambda(y)$.

-If $\operatorname{ran}_{\infty}(x)=\{1,3\}$, or $\operatorname{ran}_{\infty}(x)=\{1,2,3\}$, then $\lambda(x)=\emptyset \subset \lambda(y)$.

- If $\operatorname{ran}_{\infty}(x)=\{1,2\}$, then $\lambda(x)=\{2\}$. Also, $\operatorname{ran}_{\infty}(y) \in\{\{1\},\{2\},\{1,2\}\}$. So, $\lambda(y) \in\{\{1,2\},\{2,3\},\{2\}\}$, and, in each case, $\lambda(x) \subset \lambda(y)$.

- If $\operatorname{ran}_{\infty}(x)=\{2,3\}$, then $\lambda(x)=\{3\}$, so $\operatorname{ran}_{\infty}(y) \in\{\{2\},\{3\},\{2,3\}\}$, which implies that $\lambda(y) \in\{\{2,3\},\{3\}\}$, and, in each case, $\lambda(x) \subset \lambda(y)$.

This finishes the proof of Claim 1 .

Claim 2. The operator $\lambda$ satisfies (L3).

Proof of Claim 2. We will prove that for each $x \in X^{w}$ we have

$$
(\forall y \prec x \exists z \prec y a \in \lambda(z)) \Rightarrow a \in \lambda(x),
$$


or equivalently

$$
a \notin \lambda(x) \Rightarrow(\exists y \prec x \forall \forall \prec y a \notin \lambda(z)) .
$$

Let $x \in X^{\omega}$ and $a \notin \lambda(x)$.

. If $\left|\operatorname{ran}_{\infty}(x)\right|=1$, then also, $\left|\operatorname{ran}_{\infty}(y)\right|=\left|\operatorname{ran}_{\infty}(z)\right|=1$, which implies that $\lambda(x)=\lambda(y)=\lambda(z)$. Therefore $a \notin \lambda(x)$ implies $a \notin \lambda(z)$ witnessing (2.4).

. If $\operatorname{ran}_{\infty}(x)=\{1,2\}$, then $\lambda(x)=\{2\}$. Then $a \in\{1,3\}$. For $a=1$, let $y \prec x$ be such that $y=\langle 2\rangle$, which implies that for each $z \prec y, z=\langle 2\rangle$, i.e. $\lambda(z)=\{2,3\} \not \supset 1$. For $a=3$, let $y \prec x$ be such that $y=\langle 1\rangle$, which implies that for each $z \prec y, z=\langle 1\rangle$, i.e. $\lambda(z)=\{1,2\} \not \supset 3$.

- If $\operatorname{ran}_{\infty}(x)=\{1,3\}$ or $\operatorname{ran}_{\infty}(x)=\{1,2,3\}$ then $\lambda(x)=\emptyset$, so $a \in$ $\{1,2,3\}$. For $a=1$ or $a=2$, let $y=\langle 3\rangle$. Obviously, for each $z \prec y$ we have $z=\langle 3\rangle$ and $a \notin \lambda(z)=\{3\}$. For $a=3$, let $y=\langle 1\rangle$. Then for each $z \prec y$ we have $z=\langle 1\rangle$ and $3 \notin \lambda(z)=\{1,2\}$.

- If $\operatorname{ran}_{\infty}(x)=\{2,3\}$, then $a \in\{1,2\}$. For $y=\langle 3\rangle$, since for each $z \prec y$ we have $z=\langle 3\rangle$, we obtain $1,2 \notin \lambda(z)$.

This finishes the proof of Claim 2.

Now we reconstruct the topology $O_{\lambda}$ determined by the operator $\lambda$. According to Theorem 2.3.4, $\mathcal{F}_{\lambda}=\left\{F \subset X: \forall x \in F^{\omega} \lambda(x) \subset F\right\}$ is the corresponding family of closed sets. So, if $1 \in F \in \mathcal{F}_{\lambda}$, then $\lambda(\langle 1\rangle)=\{1,2\} \subset=F$ thus $2 \in F$. Consequently, $\{1\},\{1,3\} \notin \mathcal{F}_{\lambda}$. Similarly $2 \in F \in \mathcal{F}_{\lambda}$ implies $3 \in F$ and hence $\{2\},\{1,2\} \notin \mathcal{F}_{\lambda}$. Since $\lambda(\langle 3\rangle)=\{3\}$ we have $\{3\} \in \mathcal{F}_{\lambda}$. Since for each $x \in\{2,3\}^{\omega}$ we have $\lambda(x) \in\{\{2,3\},\{3\}\}$, implying $\lambda(x) \subset\{2,3\}$, we have $\{2,3\} \in \mathcal{F}_{\lambda}$. Thus $O_{\lambda}=\left\{\emptyset_{i}\{1\},\{1,2\},\{1,2,3\}\right\}$.

Finally, since $X$ is the only neighborhood of the point 3 , we have $3 \in \lim _{\mathcal{O}_{\lambda}}(\langle 1\rangle)$ although $3 \notin \lambda(\langle 1\rangle)$, which implies that $\lambda \neq \lim _{\mathcal{O}_{\lambda}}$.

\subsubsection{Closures of an operator $\lambda$ under (L1), (L2) and (L3)}

According to Theorem 2.2.7 if $\langle X, \mathcal{O}\rangle$ is a topological space and $\lambda=$ limo $_{\mathcal{O}}$ : $X^{\omega} \rightarrow P(X)$ corresponding limit operator then

(L1) $\forall a \in X a \in \lambda(\langle a\rangle)$;

(L2) $\forall x \in X^{\omega} \forall f \in \omega^{\dagger \omega} \lambda(x) \subset \lambda(x \circ f)$;

(L3) $\forall x \in X^{\omega}\left(\forall f \in \omega^{\dagger \omega} \exists g \in \omega^{\dagger \omega} a \in \lambda(x \circ f \circ g) \Rightarrow a \in \lambda(x)\right)$.

So, if we minimally close an a priori limit operator $\lambda: X^{\omega} \rightarrow P(X)$ under (L1), (L2) or (L3), we can expect that the obtained operator will be still included in $\lim _{\mathcal{O}_{\lambda}}$ and that the corresponding topology will coincide with $\mathcal{O}_{\lambda}$. 
An a priori limit operator $\lambda$ can be easily closed to fulfill condition (L1) adding an element $a$ to each set of the form $\lambda(\langle a, a, \ldots\rangle)$. Formally, let $\lambda^{\prime}: X^{\omega} \rightarrow P(X)$ be defined by

$$
\lambda^{\prime}(x)= \begin{cases}\lambda(x) \cup\{a\} & \text { if } x=\langle a\rangle \text { for some } a \in X, \\ \lambda(x) & \text { otherwise. }\end{cases}
$$

Clearly, $\lambda^{\prime}$ is the minimal closure of $\lambda$ under (L1).

The closure under (L2) and (L3) is considered in the sequel.

Lemma 2.3.6 Let $\lambda$ be an a priori limit operator satisfying (L1). Then $\lambda$ fulfils conditions (L2) and (L3) iff for each sequence $x \in X^{\omega}$ there holds

$$
\lambda(x)=\bigcap_{f \in \omega \mid \omega} \lambda(x \circ f)=\bigcap_{f \in \omega T \omega} \bigcup_{g \in \omega T \omega} \lambda(x \circ f \circ g) .
$$

Proof. $(\Rightarrow$ ) Let $\lambda$ fulfil (L2) and (L3). Then, for each sequence $x$ we have

$$
\bigcap_{f \in \omega^{i \omega}} \bigcup_{g \in \omega t \omega} \lambda(x \circ f \circ g) \stackrel{(\mathrm{L} 3)}{\complement} \lambda(x) \stackrel{(\mathrm{L} 2)}{\complement} \bigcap_{f \in \omega^{T \omega}} \lambda(x \circ f) .
$$

Since id $\mathrm{id}_{\omega}$ is a strictly increasing function and $x \circ f=x \circ f \circ \mathrm{id}_{\omega}$ we have

$$
\bigcap_{f \in \omega^{\prime \omega}} \lambda(x \circ f) \subset \bigcap_{f \in \omega^{\dagger \omega}} \bigcup_{g \in \omega} \lambda(x \circ f \circ g),
$$

which, together with (2.6), gives the desired equality.

$\Leftrightarrow$ Since for each $x \in X^{\omega}$ we have $\lambda(x)=\bigcap_{f \in \omega \tau} \lambda(x \circ f)$, this implies $\lambda(x) \subset \bigcap_{f \in \omega^{\text {Tw }}} \lambda(x \circ f), x \in X^{\omega}$, which is equivalent to (L2). Also $\lambda(x)=$ $\bigcap_{f \in \omega^{t} \omega} \bigcup_{g \in \omega^{t \omega}} \lambda(x \circ f \circ g), x \in X^{\omega}$ implies $\lambda(x) \supset \bigcap_{f \in \omega t \omega} \bigcup_{g \in \omega t \omega} \lambda(x \circ f \circ g)$, $x \in X^{\omega}$, a condition equivalent to (L3).

Now, let us close an a priori limit operator to fulfill (L2).

Theorem 2.3.7 For an a priori limit operator $\lambda: X^{\omega} \rightarrow P(X)$ satisfying (L1), let $\bar{\lambda}: X^{\omega} \rightarrow P(X)$ be the a priori limit operator defined by

$$
\bar{\lambda}(x)=\bigcup_{y \in X^{\omega}, f \in \omega^{\top} \omega, x=y \circ f} \lambda(x) .
$$

Then

(a) $\lambda(x) \subset \bar{\lambda}(x)$ for each $x \in X^{\omega}$;

(b) $\bar{\lambda}$ satisfies conditions (L1) and (L2);

(c) $\bar{\lambda}$ is the minimal operator satisfying (a) and (b). 
Proof. (a) Let $x \in X^{\omega}$. For $f=\mathrm{id}_{\omega}$, by the definition of $\bar{\lambda}$, we conclude that $\lambda(x \circ f)=\lambda(x) \subset \bar{\lambda}(x)$.

(b) From (a) it follows that $\bar{\lambda}$ satisfies (L1). Let us prove that $\vec{\lambda}$ satisfies (L2), which is eqivalent to the fact that for each sequence $y$ and each $g \in \omega^{\top \omega}$ we have

$$
\bar{\lambda}(x) \subset \bar{\lambda}(x \circ g) .
$$

Let $a \in \bar{\lambda}(x)$. Then there exist $y \in X^{\omega}$ and $f \in \omega^{\dagger \omega}$ such that $x=y \circ f$ and $a \in \lambda(y)$. Since $x \circ g=y \circ f \circ g$, and since $f \circ g \in \omega^{\dagger \omega}$, we have that $a \in \lambda(y) \subset \bar{\lambda}(x \circ g)$, which proves (2.8).

(c) Let us suppose that $\lambda_{1}$ is an a priori limit operator satisfying (L1) and (L2) such that $\lambda \leq \lambda_{1}$. Let us prove that $\bar{\lambda} \leq \lambda_{1}$. Let $x$ be a sequence in $X$ and $a \in \bar{\lambda}(x)$. Then, there exist $y \in X^{\omega}$ and $f \in \omega^{\uparrow \omega}$ such that $x=y \circ f$ and $a \in \lambda(y)$. Then, from $\lambda \leq \lambda_{1}$ it follows that $a \in \lambda_{1}(y)$, and, since $\lambda_{1}$ fulfils (L2), $a \in \lambda_{1}(x)$. Thus $\bar{\lambda} \leq \lambda_{1}$.

Now, let us close an a priori operator $\lambda$ satisfying (L1) and (L2) under (L3).

Theorem 2.3.8 For an a priori limit operator $\lambda$ satisfying (L1) and (L2), let $\lambda^{*}$ : $X^{\omega} \rightarrow P(X)$ be the a priori limit operator defined by

$$
\lambda^{*}(x)=\bigcap_{f \in \omega^{\top \omega}} U_{g \in \omega^{\top \omega}} \lambda(x \circ f \circ g) .
$$

Then

(a) $\lambda(x) \subset \lambda^{*}(x)$ for each $x \in X^{\omega}$.

(b) $\lambda^{*}$ satisfies conditions (L1), (L2) and (L3).

(c) $\lambda^{*}$ is the minimal operator satisfying (a) and (b).

Proof. (a) Let $a \in \lambda(x)$ and $f \in \omega^{\top \omega}$. Since $\mathrm{id}_{\omega} \in \omega^{\top \omega}$ and by property (L2) of $\lambda$ we have $a \in \lambda(x) \subset \lambda(x \circ f)=\lambda\left(x \circ f \circ i d_{\omega}\right) \subset \lambda^{*}(x)$.

(b) By (a), it is obvious that $\lambda^{*}$ fulfils (L1). Let us prove that $\lambda^{*}$ fulfils (L2). Let $x$ be a sequence in $X, a \in \lambda^{*}(x)$ and $h \in \omega^{\uparrow \omega}$. We will show that $a \in \lambda^{*}(x \circ h)$, which is, by the definition of $\lambda^{*}$, equivalent to

$$
\forall \varphi \in \omega^{\uparrow \omega} \exists g \in \omega^{\uparrow \omega} a \in \lambda(x \circ h \circ \varphi \circ g) .
$$

Since $a \in \lambda^{*}(x)$ there holds

$$
\forall f \in \omega^{T \omega} \exists g \in \omega^{i \omega} a \in \lambda(x \circ f \circ g) .
$$

For $\varphi \in \omega^{\dagger \omega}$ we have $h \circ \varphi \in \omega^{\dagger \omega}$. Therefore, equation (2.11), for $f=h \circ \varphi$. implies that there exists $g \in \omega^{\dagger \omega}$ such that $a \in \lambda(x \circ f \circ g)=\lambda(x \circ h \circ \varphi \circ g)$, witnessing (2.10). 
Now, let us prove that $\lambda^{*}$ satisfies (L3). Let $x$ be a sequence in $X$ and $a \in X$ such that

$$
\forall f \in \omega^{\top \omega} \exists g \in \omega^{\top \omega} a \in \lambda^{*}(x \circ f \circ g) .
$$

This is equivalent to

$$
\forall f \in \omega^{\uparrow \omega} \exists g \in \omega^{\dagger \omega} \forall F \in \omega^{\uparrow \omega} \exists G \in \omega^{\uparrow \omega} a \in \lambda(x \circ f \circ g \circ F \circ G) .
$$

Let us prove that $a \in \lambda^{*}(x)$, i.e.

$$
\forall \varphi \in \omega^{\dagger \omega} \exists \psi \in \omega^{\dagger \omega} a \in \lambda(x \circ \varphi \circ \psi) .
$$

For each $\varphi \in \omega^{\top \omega}$, by (2.12) there exists $g \in \omega^{\top \omega}$ such that

$$
\forall F \in \omega^{\uparrow \omega} \exists G \in \omega^{\uparrow \omega} a \in \lambda(x \circ \varphi \circ g \circ F \circ G) .
$$

So, for $F=\operatorname{id}_{\omega}$ there exists $G \in \omega^{\dagger \omega}$ such that $a \in \lambda(x \circ \varphi \circ g \circ G)$. The fact that $g \circ G \in \omega^{i \omega}$ completes the proof of (2.13).

(c) Let us suppose that $\lambda_{1}$ is an a priori limit operator satisfying (L1), (L2) and (L3) such that $\lambda \leq \lambda_{1}$. Let us prove that $\lambda^{*} \leq \lambda_{1}$. Let $x$ be a sequence in $X$ and $a \in \lambda^{*}(x)$. Then, for each $f \in \omega^{\top \omega}$ there exists $g \in \omega^{\dagger \omega}$ such that $a \in \lambda(x \circ f \circ g)$. By $\lambda \leq \lambda_{1}$, we have that $a \in \lambda_{1}(x \circ f \circ g)$, and, since $\lambda_{1}$ fulfils (L3), it implies that $a \in \lambda_{1}(x)$. Thus $\lambda^{*} \leq \lambda_{1}$.

Theorem 2.3.9 Let $\lambda: X^{\omega} \rightarrow P(X)$ be an a priori limit operator satisfying (L1) and (L2) such that for each $x \in X^{\omega}$ we have $|\lambda(x)| \leq 1$. Then for each $x \in X^{\omega}$ we have $\left|\lambda^{*}(x)\right| \leq 1$.

Proof. Let us suppose that there exist $x \in X^{\omega}$ and $a, b \in X$ such that $a, b \in \lambda^{*}(x)$. Since $\mathrm{id}_{\omega} \in \omega^{\lceil\omega}$, by (2.9), there exists $g_{a} \in \omega^{\top \omega}$ such that $a \in \lambda\left(x \circ \mathrm{id}_{\omega} \circ g_{a}\right)=$ $\lambda\left(x \circ g_{a}\right)$. Also, by (2.9), there exists $g_{b}$ such that $b \in \lambda\left(x \circ g_{a} \circ g_{b}\right)$.

Since $x \circ g_{a} \circ g_{b} \prec x \circ g_{a}$ and $\lambda$ satisfies (L2) we have that $a \in \lambda\left(x \circ g_{a} \circ g_{b}\right)$. Since $\left|\lambda\left(x \circ g_{a} \circ g_{b}\right)\right| \leq 1$ we have that $a=b$.

Let $\lambda: X^{\omega} \rightarrow P(X)$ be an arbitrary a priori limit operator. If we close it under (L1), then under (L2) and, finally, under (L3), we obtain the sequence of operators $\lambda \leq \lambda^{\prime} \leq \lambda^{\prime-} \leq \lambda^{\prime-*}$. The last one fulfils conditions (LI)-(L3). Let us prove that all these four a priori operators generate the same maximal topology in the sense of Theorem 2.3.1,

Theorem 2.3.10 Let $\lambda: X^{\omega} \rightarrow P(X)$ be an a priori limit operator. Then $\mathcal{O}_{\lambda}=$ $\mathcal{O}_{\lambda^{\prime}}=\mathcal{O}_{\lambda^{\prime-}}=\mathcal{O}_{\lambda^{\prime}-\ldots}$ 
Proof. By Theorem 2.2.7, the operator $\lim _{\mathcal{O}_{\lambda}}$ satisfies (L1)-(L3). From $\lambda \leq \lim _{\mathcal{O}_{\lambda}}$ and the fact that $\lambda^{\prime}$ is the minimal closure of $\lambda$ under (L1) it follows that $\lambda^{\prime} \leq$ $\lim _{\mathcal{O}_{\lambda}}$. Similarly, from the minimality of $\lambda^{\prime-}$ (Theorem 2.3.7 (c)) it follows $\lambda^{\prime-} \leq$ $\lim _{\mathcal{O}_{\lambda}}$. Finally, by Theorem 2.3 .8 (c), it follows $\lambda^{\prime-*} \leq \lim _{\mathcal{O}_{\lambda}}$. So, $\lambda \leq \lambda^{\prime} \leq$ $\lambda^{\prime-} \leq \lambda^{\prime-} \leq \lim _{\mathcal{O}_{\lambda}}$, which, by Lemma 2.3.2, implies $\mathcal{O}_{\lambda} \supset \mathcal{O}_{\lambda^{\prime}} \supset \mathcal{O}_{\lambda^{\prime-}} \supset$

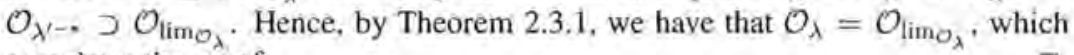
completes the proof.

\subsubsection{The operators $u_{\lambda}$ and $\mathrm{cl}_{\omega_{1}}$}

For an a priori limit operator $\lambda: X^{\omega} \rightarrow P(X)$, we found the maximal topology on the set $X$ with property (2.2), $\mathcal{O}_{\lambda}$. If $\lambda$ satisfies (L1) and (L2) this topology is determined by the family of closed sets, as in Theorem 2.3.4. In this subsection we will describe the topological closure operator in the space $\left\langle X, \mathcal{O}_{\lambda}\right\rangle$ in terms of $\lambda$.

Definition 2.3.11 For an a priori limit operator $\lambda: X^{\omega} \rightarrow P(X)$ let $u_{\lambda}: P(X) \rightarrow$ $P(X)$ be the operator defined by

$$
u_{\lambda}(A)=\bigcup_{y \in A^{\omega}} \lambda(y)+
$$

When it is clear which a priori limit operator is used, instead of $u_{\lambda}$ we will briefly write $u$.

Lemma 2.3.12 If an a priori limit operator $\lambda$ satisfies conditions (L1) and (L2) then

(a) $u(\emptyset)=\emptyset$;

(b) $A \subset u(A)$;

(c) $A \subset B \Rightarrow u(A) \subset u(B)$;

(d) $u(A \cup B)=u(A) \cup u(B)$.

Proof. Assertions (a) and (c) are obvious, and (b) follows from condition (L1). Let us prove (d). From (c) it follows that $u(A) \cup u(B) \subset u(A \cup B)$. Let $a \in u(A \cup B)$. Then there exists a sequence $y \in(A \cup B)^{\omega}$ such that $a \in \lambda(y)$. Clearly, there exists a subsequence $x \prec y$ such that $x \in A^{\omega}$ or $x \in B^{\omega}$. Since $\lambda$ fulfils (L2), we have that $a \in \lambda(x)$. In the first case, $a \in u(A)$, and in the second, $a \in u(B)$.

Theorem 2.3.13 Let $\lambda$ be an a priori limit operator satisfying (L1) and (L2) and let $A \subset X$. Then the following conditions are equivalent:

(a) The set $A \subset X$ is closed in the topology $\mathcal{O}_{\lambda}$ :

(b) $A=u(A)$;

(c) $\lambda$-limit of each sequence in $A$ is a subset of $A$. 
Proof. By Theorem 2.3.4, we have that (a) is equivalent to (c), and that (a) is equiyalent to $u(A) \subset A$. Since, by Lemma 2.3.12 (b), we have $A \subset u(A)$, there holds equivalence (a) and (b).

Let us notice that the operator $u$ must not be a closure operator in general. since $u(A)$ is not always equal to $u(u(A))$ (property $(\mathrm{CO} 4)$ ). Iterating operator $u$ $\omega_{1}$-times we obtain a new operator.

Theorem 2.3.14 Let $\lambda$ be an a priori limit operator which satisfies conditions (L1) and (L2). Let $\mathrm{cl}_{\alpha}: P(X) \rightarrow P(X), \alpha \leq \omega_{1}$, be a family of mappings such that

$\cdot \operatorname{cl}_{0}(A)=A$

$\cdot \mathrm{cl}_{\alpha+1}(A)=u\left(\mathrm{cl}_{\alpha}(A)\right)$

$\cdot \mathrm{cl}_{\gamma}(A)=\bigcup_{\alpha<\gamma} \mathrm{cl}_{\alpha}(A)$, for the limit ordinal $\gamma$.

Then $\mathrm{cl}_{\omega_{1}}$ is the closure operator on the topological space $\left\langle X, \mathcal{O}_{\lambda}\right\rangle$, i.e. for each $A \subset X$ we have $\mathrm{cl}_{\omega_{1}}(A)=\bar{A}^{\lambda}$.

Proof. By Theorem 2.3.13, a set $F$ is closed iff $u(F)=F$. Therefore, it is sufficient to prove that for each set $A$, the set $\operatorname{cl}_{\omega_{1}}(A)$ is the smallest closed set containing $A$.

Let us show that $A \subset \operatorname{cl}_{\omega_{1}}(A)$. We have that $A=\operatorname{cl}_{0}(A)$. Let us suppose that for each $\alpha<\beta$ we have $A \subset \operatorname{cl}_{\alpha}(A)$. If $\beta=\gamma+1$, then, by Lemma 2.3.12 (b), $A \subset \operatorname{cl}_{\gamma}(A) \subset u\left(\operatorname{cl}_{\gamma}(A)\right)=\operatorname{cl}_{\beta}(A)$. If $\beta$ is a limit ordinal, then $A \subset \bigcup_{\alpha<\beta} \operatorname{cl}_{\alpha}(A)=\operatorname{cl}_{\beta}(A)$.

Let us show that $u\left(\mathrm{cl}_{\omega_{1}}(A)\right)=\mathrm{cl}_{\omega_{1}}(A)$. By Lemma 2.3.12 (b), $\mathrm{cl}_{\omega_{1}}(A) \subset$ $u\left(\mathrm{cl}_{\omega_{1}}(A)\right)$. Let $x$ be a sequence in $\operatorname{cl}_{\omega_{1}}(A)=\bigcup_{\alpha<\omega_{1}} \mathrm{cl}_{\alpha}(A)$. Then, there exists $\beta<\omega_{1}$ such that $x$ is a sequence in $\operatorname{cl}_{\beta}(A)$, which implies that $\lambda(x) \subset$ $u\left(\operatorname{cl}_{\beta}(A)\right)=\operatorname{cl}_{\beta+1}(A) \subset \operatorname{cl}_{\omega_{1}}(A)$.

Let us prove the minimality of $\mathrm{el}_{\omega_{1}}(A)$. Let $F$ be a closed set containing $A$. Then, by Theorem 2.3.13, $u(F)=F$. We will prove that for each $\alpha \leq \omega_{1}$ we have $\mathrm{cl}_{\alpha}(A) \subset F$. For $\alpha=0$ we have $\operatorname{cl}_{0}(A)=A \subset F$. Let us suppose that for each $\beta<\alpha$ we have $\operatorname{cl}_{\beta}(A) \subset F$. If $\alpha=\gamma+1$, from $\operatorname{cl}_{\gamma}(A) \subset F$ and by Lemma 2.3.12 (c), we have $\mathrm{cl}_{\alpha}(A)=\mathrm{ch}_{\gamma+1}(A)=u\left(\operatorname{cl}_{\gamma}(A)\right) \subset u(F)=F$. If $\alpha$ is a limit ordinal, then $\operatorname{cl}_{\alpha}(A)=\bigcup_{\beta<\alpha} \mathrm{cl}_{\beta}(A) \subset \bigcup_{\beta<\alpha} F=F$.

Theorem 2.3.15 Let $\lambda$ be an a priori limit operator with properties (L1) and (L2). Then the topological space $\left\langle X, \mathcal{O}_{\lambda}\right\rangle$ is sequential, i.e.

$$
A \in \mathcal{F}_{\lambda} \Leftrightarrow \forall y \in A^{\omega} \lim _{O_{\lambda}}(y) \subset A .
$$

Proof. $(\Rightarrow$ ) holds in each topological space. 
$(\Leftrightarrow)$ Let for each $y \in A^{\omega}$ we have $\lim _{\mathcal{O}_{\lambda}}(y) \subset A$. According to Theorem 2.3.13 we prove $A=u(A)$. Let $a \in u(A)$. Then there exists a sequence $y$ in $A$ such that $a \in \lambda(y)$. Since $\lambda(y) \subset \lim _{\mathcal{O}_{\lambda}}(y) \subset A$ we have $a \in A$. Thus $u(A) \subset A$ and, clearly, $A \subset u(A)$.

\subsubsection{The equality $u^{2}=u$. Fréchet spaces}

Theorem 2.3.16 If an a priori limit operator $\lambda: X^{\omega} \rightarrow P(X)$ satisfies conditions (L1) and (L2), then the following conditions are equivalent

(a) $u^{2}=u$;

(b) $u=\mathrm{cl}_{\omega_{1}}$;

(c) The operator $\lambda$ satisfies condition (L4).

Proof. $\left((\mathrm{a}) \Rightarrow\right.$ (b)) Let $u^{2}=u$. Using induction it is easy to prove that for each $\alpha \leq \omega_{1}$ for each $A \subset X$ there holds $\operatorname{cl}_{\alpha}(A) \subset u(A)$ so $\operatorname{cl}_{\omega_{1}}(A) \subset u(A)$ and the reversed inclusion is trivial.

(b) $\Rightarrow$ (c). Suppose $u(A)=\operatorname{cl}_{\omega_{1}} A$, for all $A \subset X$. Let $a \in \lambda\left(\left\langle x_{n}: n \in \omega\right\rangle\right)$ and for each $n \in \omega$ let $x_{n} \in \lambda\left(\left\langle y_{k}^{n}: k \in \omega\right\rangle\right)$. If $Y=\left\{y_{k}^{n}: n, k \in \omega\right\}$, then for each $n \in \omega$ there holds $x_{n} \in u(Y)$ so $x \in u(Y)^{\omega}$ and consequently $a \in \lambda(x) \subset u(u(Y))=\operatorname{cl}_{\omega_{1}}\left(\mathrm{cl}_{\omega_{1}}(Y)\right)=\mathrm{cl}_{\omega_{1}}(Y)=u(Y)$ thus there is $z \in Y^{\omega}$ such that $a \in \lambda(z)$.

(c) $\Rightarrow$ (a). Suppose $\lambda$ satisfies (L4). For $A \subset X$ we prove $u(u(A)) \subset u(A)$. Let $a \in u(u(A))$. Then there is $x \in u(A)^{\omega}$ such that $a \in \lambda(x)$. For each $n \in \omega$ we have $x^{n} \in u(A)$ hence there is $\left\langle y_{k}^{n}: k \in \omega\right\rangle \in A^{\omega}$ such that $x^{n} \in \lambda\left(\left\langle y_{k}^{n}: k \in \omega\right\rangle\right)$. By (L4) there is $z \in\left\{y_{k}^{n}: n, k \in \omega\right\}^{\omega} \subset A^{\omega}$ such that $a \in \lambda(z)$ so, since $z \in A^{\omega}$, we have $a \in u(A)$.

Corollary 2.3.17 For an a priori operator $\lambda: X^{\omega} \rightarrow P(X)$ the following conditions are equivalent.

(a) There exists $A \subset X$ such that $u(u(A)) \neq u(A)$.

(b) There exist set $\left\{x_{i}^{n}: n, i \in \omega\right\} \subset X$, sequence $\left\langle x^{n}: n \in \omega\right\rangle \in X^{\omega}$ and element $b \in X$ such that $b \in \lambda\left(\left\langle x^{n}: n \in \omega\right\rangle\right), x^{n} \in \lambda\left(\left\langle x_{i}^{n}: i \in \omega\right\rangle\right), n \in \omega$, and for each $x \in Z^{\omega}$ we have $b \notin \lambda(x)$.

Theorem 2.3.18 If for each $A \subset X$ we have $u(u(A))=u(A)$ then the space $\left\langle X, \mathcal{O}_{\lambda}\right\rangle$ is a Fréchet space.

Proof. Let $A \subset X$ and let $b \in \mathrm{cl}_{\omega_{1}}(A)$. By Theorem 2.3.16, we have $b \in u(A)$. So, there exists a sequence $x$ in $A$ such that $b \in \lambda(x) \subset \lim _{\mathcal{O}_{\lambda}}(x)$.

The inverse implication must not be true. 
Example 2.3.19 A Frechét space in which $u_{\lambda}^{2} \neq u_{\lambda}$.

Let us consider a set $X=\{1,2,3\}$ and the a priori limit operator $\lambda$ defined in Example 2.3.5. We have shown that $\mathcal{O}_{\lambda}=\{\emptyset,\{1\},\{1,2\}, X\}$. We will prove that $\left\langle X, \mathcal{O}_{\lambda}\right\rangle$ is a Frechét space, i.e.

$$
\forall A \subset X \forall b \in c l_{\omega_{1}}(A) \backslash A \exists x \in A^{\omega} b \in \lim _{\mathcal{O}_{\lambda}} x
$$

Let us notice that the only open set containing 3 is the whole set $X$, and therefore each sequence converges to the point 3 . So, we will prove that formula (2.14) is equivalent to

$$
\forall A \subset X \forall b \in c_{\omega_{1}}(A) \backslash(A \cup\{3\}) \exists x \in A^{\omega} b \in \lim _{O_{\lambda}} x
$$

Obviously, from (2.14) it follows (2.15). Let us prove the inverse implication. Let $A \subset X$ and $b \in c l_{\omega_{1}}(A) \backslash A$. For $b \neq 3$ the implication is obvious. If $b=3$ for any $a \in A$ we have $3 \in \lim _{O_{\lambda}}\langle a\rangle$.

It is easy to verify that the only two sets such that $c l_{\omega_{1}}(A) \backslash(A \cup\{3\})$ is non-empty are $\{1\}$ and $\{1,3\}$. The closure of both of them is the set $X$. For both sets we have $c_{\omega_{1}}(A) \backslash(A \cup\{3\})=\{2\}$ and both of them contain 1. Since $\lambda(\langle 1\rangle)=\{1,2\}$, we have $2 \in \lambda(\langle 1\rangle) \subset \lim _{\mathcal{O}_{\lambda}}\langle 1\rangle$, which proves that the space is Frechét. But,

$$
\begin{gathered}
u(\{1\})=\bigcup_{x \in\{1\} \sim} \lambda(x)=\lambda(\langle 1\rangle)=\{1,2\} . \\
u(u(\{1\}))=\bigcup_{x \in\{1,2\} \omega} \lambda(x)=\{1,2\} \cup\{2,3\} \cup\{2\}=\{1,2,3\} .
\end{gathered}
$$

This implies that $u(u(\{1\})) \neq u(\{1\})$.

On the set $X$, using different a priori limit operators, we can generate several sequential topologies.

Theorem 2.3.20 Let $\lambda_{1}$ and $\lambda_{2}$ be a priori limit operators on $X$ satisfying (L1). Let $\bar{\lambda}_{1}$ and $\bar{\lambda}_{2}$ be operators defined in Theorem 2.3.7, and $u_{\bar{\lambda}_{1}}$ and $u_{\bar{\lambda}_{2}}$ be operators defined in Definition 2.3.11 using $\bar{\lambda}_{1}$ and $\bar{\lambda}_{2}$. Let $\mathrm{cl}_{\underline{\omega}_{1}}^{1}$ and $\mathrm{cl}_{\omega_{1}}^{1}$ be closure operators defined in Theorem 2.3.14 using operators $\bar{\lambda}_{1}$ and $\bar{\lambda}_{2}$ respectively.

If $\lambda_{1} \leq \lambda_{2}$ then

(a) $\bar{\lambda}_{1} \leq \bar{\lambda}_{2}$;

(b) $u_{\bar{\lambda}_{1}}(A) \subset u_{\bar{\lambda}_{2}}(A)$, for each $A \subset X$;

(c) $\mathrm{cl}_{w_{1}}^{1}(A) \subset \mathrm{cl}_{\omega_{1}}^{2}(A)$, for each $A \subset X$;

(d) $\mathcal{O}_{\lambda_{2}} \subset \mathcal{O}_{\lambda_{1}}$;

(e) $\lim _{\lambda_{1}} \leq \lim _{\lambda_{2}}$. 


\section{Proof.}
(a) $\bar{\lambda}_{1}(x)=\bigcup_{x \prec y} \lambda_{1}(y) \subset \bigcup_{x \prec y} \lambda_{2}(y)=\bar{\lambda}_{2}$.
(b) $u_{\bar{\lambda}_{1}}(A)=\bigcup_{y \in A^{\omega}} \bar{\lambda}_{1}(y) \subset \bigcup_{y \in A^{\omega}} \bar{\lambda}_{2}(y)=u_{\bar{\lambda}_{2}}(A)$.
(c) Can be easily obtained by induction using (b).
(d) This is Theorem 2.3.2.
(e) Follows directly from (d) and Fact 2.2.3. 
The Good Lord made all the integers;

the rest is man's doing.

Leopold Kronecker

\section{Chapter 3}

\section{Boolean algebras}

In this chapter we give some elementary information about Boolean algebras with the accent on the properties of algebraic convergence of sequences.

\subsection{Definitions and basic facts}

On a Boolean algebra $\left\langle\mathbb{B}, \wedge, \vee^{\prime}, 0,1\right\rangle$ the partial order $\leq$ is defined by

$$
a \leq b \Leftrightarrow a \wedge b=a .
$$

Then, the supremum (infimum) of a two element set $\{a, b\}$ is $a \vee b(a \wedge b)$. The supremum (infimum) of a set $A \subset \mathbb{B}$ (if it exists) is denoted by $\bigvee_{a \in A} a\left(\bigwedge_{a \in A} a\right)$ or just by $\bigvee A(\bigwedge A)$.

$\mathbb{B}^{+}$denotes the set of all positive elements of $\mathbb{B}$, i.e. $\mathbb{B}^{+}=\mathbb{B} \backslash\{0\}$. An element $b \in \mathbb{B}^{+}$is an atom iff it is a minimal element of $\mathbb{B}^{+}$. A Boolean algebra $\mathbb{B}$ is atomic iff for each $a \in \mathbb{B}^{+}$there exists an atom $b$ such that $b \leq a$. A set $A \subset \mathbb{B}$ is a wellordered chain iff there exists an ordinal $\alpha$ such that $A=\left\{a_{\beta}: \beta<\alpha\right\}$ and for $\beta<\gamma<\alpha$ we have $a_{\beta}<a_{\gamma}$. A set $A \subset \mathbb{B}^{+}$is an antichain iff for each different $a, b \in A$ there holds $a \wedge b=0$. For a cardinal $\kappa, \mathbb{B}$ satisfies the $\kappa-c c$ ( $\kappa$ chain condition) iff $\mathbb{B}$ does not contain $\kappa$-sized antichains. In particular, instead of $\aleph_{1}-\mathrm{ec}$ we write $\operatorname{ccc}$ (the countable chain condition). A set $A \subset \mathbb{B}$ is a quasi-partition of an element $c$ iff for each different $a, b \in A$ there holds $a \wedge b=0$ and $\bigvee A=c$. A quasi-partition $A$ of $c \in \mathbb{B}^{+}$is a partition of $c$ iff $A \subset \mathbb{B}^{+}$. A Boolean algebra is 
$\kappa$-complete iff each set $A \subset \mathbb{B}$ of size less than $\kappa$ has the supremum (equivalently, the infimum). A Boolean algebra is a $\sigma$-algebra iff it is an $\aleph_{1}$-complete algebra. A Boolean algebra is complete iff each set $A \subset \mathbb{B}$ has the supremum. Often, instead of "complete Boolean algebra", the abbreviation c.B.a. will be used. The power set of a cardinal $\kappa,\left\langle P(\kappa), \cap, \cup,{ }^{c}, \emptyset, \kappa\right\rangle$, is an example of a complete atomic Boolean algebra and for each atomic c.B.a. $\mathbb{B}$ there exists $\kappa$ such that $\mathbb{B}$ is isomorphic to $P(\kappa)$.

For a set $A \subset \mathbb{B}$ let

$$
A \uparrow=\{b \in \mathbb{B}: \exists a \in A a \leq b\} \text { and } A \downarrow=\{b \in \mathbb{B}: \exists a \in A a \geq b\} .
$$

Instead of $\{b\} \uparrow$ and $\{b\} \downarrow$ we write $b \uparrow$ and $b \downarrow$ respectively. Clearly, $A \uparrow=$ $\bigcup_{a \in A}(a \uparrow)$ and $A \downarrow=\bigcup_{a \in A}(a \downarrow)$.

We will say that a subset $D \subset \mathbb{B}^{+}$is dense in a Boolean algebra $\mathbb{B}$ iff $D$ is dense in the partial order $\left\langle\mathbb{B}^{+}, \leq\right\rangle$.

For more details about Boolean algebras we recommend [25], [46] and [39].

\subsection{Tall ideals}

If $\mathbb{B}$ is a Boolean algebra, a family $\mathcal{J} \subset \mathbb{B}$ is an ideal iff

(I1) $0 \in \mathcal{J} \not \supset 1 ; \quad$ (I2) $a \leq b \in \mathcal{J} \Rightarrow a \in \mathcal{J} ; \quad$ (I3) $a, b \in \mathcal{J} \Rightarrow a \vee b \in \mathcal{J}$.

A family $\Phi \subset \mathbb{B}$ is a filter iff

(F1) $1 \in \Phi \not \supset 0 ; \quad$ (F2) $a \geq b \in \Phi \Rightarrow a \in \Phi ; \quad$ (F3) $a, b \in \Phi \Rightarrow a \wedge b \in \Phi$.

If $\mathcal{J}$ is an ideal in $\mathbb{B}$, then the set $\mathcal{J}^{*}=\left\{a^{\prime}: a \in \mathcal{J}\right\}$ is a filter in $\mathbb{B}$, called the dual filter. The dual ideal is defined similarly. A maximal filter is called an ultrafilter. A filter $\Phi$ is an ultrafilter iff for each element $b \in \mathbb{B}$ either $b \in \Phi$ or $b^{\prime} \in \Phi$.

Definition 3.2.1 Let $A$ be a countable set. An ideal $\mathcal{J} \subset P(A)$ is a tall ideal iff

$$
\forall X \in[A]^{\omega} \exists I \in \mathcal{J}|X \cap I|=\aleph_{0} .
$$

The set of all tall ideals in $P(\omega)$ will be denoted by Tall $(\omega)$.

Fact 3.2.2 (a) An ideal $\mathcal{J} \subset P(\omega)$ is a tall ideal iff $\forall X \in[\omega]^{\omega} \exists I \in \mathcal{J} \cap[\omega]^{\omega} I \subset$ $X$.

(b) If $\mathcal{J}$ is a tall ideal on $P(\omega)$ then $\mathcal{D}=\mathcal{J} \cap[\omega]^{\omega}$ is a dense set in $\left\langle[\omega]^{\omega}, C\right\rangle$ 
(c) An ideal $\mathcal{J} \subset P(\omega)$ is a tall ideal iff the dual filter $\mathcal{J}^{*}$ has no pseudointersection.

(d) If $\mathcal{U} \subset P(\omega)$ is an ultrafilter, then its dual $\mathcal{U}^{*}$ is a tall ideal.

(e) If $\mathcal{A} \subset P(\omega)$ is madf then $\mathcal{J}_{\mathcal{A}}=\left\{I \subset \omega: \exists \mathcal{F} \in[\mathcal{A}]^{<\omega} I \subset \cup \mathcal{F}\right\}$ is a tall ideal.

Example 3.2.3 Let $\mathcal{S}=\left\{S \in\left[{ }^{<\omega} 2\right]^{\omega}: S\right.$ is a chain or an antichain $\}$. Let us prove that the set

$$
\mathcal{J}_{\mathcal{S}}=\left\{I \subset<\omega_{2}: \exists \mathcal{S}_{1} \in[\mathcal{S}]^{<\omega} I \subset \bigcup \mathcal{S}_{1}\right\}
$$

is a tall ideal on $<\omega_{2}$.

One can verify that $\mathcal{J}_{S}$ is an ideal on ${ }^{</ 2}$.

Let $X \in\left[{ }^{*} \omega_{2}\right]^{\omega}$ and

$$
H_{0}=\left\{\{x, y\} \in[X]^{2}: x<y \vee y<x\right\}, \quad H_{1}=[X]^{2} \backslash H_{0}
$$

Since $[X]^{2}=H_{0} \cup H_{1}$, the Ramsey theorem (see [43]) implies that there exists $H \in[X]^{\omega}$ such that $[H]^{2} \subset H_{0}$ or $[H]^{2} \subset H_{1}$. If

- $[H]^{2} \subset H_{0}$, then all elements in $H$ are comparable, which implies that $H$ is infinite chain.

- $[H]^{2} \subset H_{1}$, then all elements in $H$ are not comparable, which implies that $H$ is infinite antichain.

Therefore $H \in \mathcal{S} \subset \mathcal{J}_{\mathcal{S}}$ and $H$ is an infinite subset of $X$, witnessing that $\mathcal{J}_{\mathcal{S}}$ is a tall ideal on $<\omega_{2}$.

\subsection{Sequences in Boolean algebras, lim sup and lim inf}

In this section some definitions concerning sequences and matrices on a Boolean algebra will be given. Also, the algebraic convergence of sequences and its basic properties will be considered.

Definition 3.3.1 A sequence $\left\langle a_{k}: k \in \omega\right\rangle$ in a Boolean algebra $\mathbb{B}$ is:

- increasing iff $a_{i} \leq a_{j}$ for $i<j$.

decreasing iff $a_{i} \geq a_{j}$ for $i<j$.

We will write $\left\langle a_{k}\right\rangle, b$ iff $\left\langle a_{k}\right\rangle$ is increasing and $\bigvee_{k \in \omega} a_{k}=b$ and $\left\langle a_{k}\right\rangle \searrow b$ iff $\left\langle a_{k}\right\rangle$ is decreasing and $\bigwedge_{k \in \omega}, a_{k}=b$.

Definition 3.3.2 A matrix $\left[a_{n k}\right] \in \mathbb{B}^{\omega \times \omega}$ is . increasing iff for each $n \in \omega$ the sequence $\left\langle a_{n k}: k \in \omega\right\rangle>1$. + decreasing iff for each $n \in \omega$ the sequence $\left\langle a_{n k}: k \in \omega\right\rangle$. 
Lemma 3.3.3 A sequence $\left\langle x_{n}\right\rangle$ is increasing iff the sequence $\left\langle x_{n}^{\prime}\right\rangle$ is decreasing. A matrix $\left[a_{n k}\right]$ is increasing iff $\left[a_{n k k}^{\prime}\right]$ is decreasing.

Definition 3.3.4 Let $x=\left\langle x_{n}: n \in \omega\right\rangle$ be a sequence in a c.B.a. $\mathbb{B}$. Then

$$
\limsup x=\bigwedge_{k \in \omega} \bigvee_{n \geq k} x_{n} \text { and } \liminf x=\bigvee_{k \in \omega} \bigwedge_{n \geq k} x_{n}
$$

Lemma 3.3.5 For each sequence $x \in \mathbb{B}^{\omega}$ there holds

$$
\lim \inf x \leq \lim \sup x
$$

Lemma 3.3.6 Let $x=\left\langle x_{n}: n \in \omega\right\rangle$ be a sequence in a c.B.a. $\mathbb{B}$ and $f \in \omega^{\omega}$. Then

(a) if $f$ is an injection, then $\liminf x \leq \liminf x \circ f \leq \limsup x \circ f \leq$ $\lim \sup x$;

(b) if $f$ is a strictly increasing function, then $\liminf x \leq \liminf x \circ f \leq$ limsup $x \circ f \leq \lim \sup x$;

(c) if $f$ is a bijection, then $\liminf x=\liminf x \circ f \leq \limsup x \circ f=$ $\limsup x$;

(d) if $f$ is "finite to one", then $\liminf x \leq \liminf x \circ f \leq \limsup x \circ f \leq$ $\lim \sup x$;

(e) if $f(n)=n+m$, then $\liminf x=\liminf x \circ f \leq \lim \sup x \circ f=\lim \sup x$.

Proof. Only the proofs for limsup will be given, since the proofs for lim inf are analogous.

(a) Let $k \in \omega$. Since $f$ is an injection, the set $A_{k}=f^{-1}[\{1,2, \ldots, k-1\}]$ is finite and therefore it has the maximum. Let $m(k)=\max A_{k}+1$. Then $\bigvee_{n \geq m(k)} x_{f(n)} \leq \bigvee_{n \geq k} x_{n}$. This implies that for each $k$ we have $\bigwedge_{l \in \omega} \bigvee_{n \geq l} x_{f(n)} \leq$ $\bigvee_{n \geq k} x_{n}$. Therefore $\bigwedge_{l \in \omega} \bigvee_{n \geq l} x_{S(n)} \leq \bigwedge_{k \in \omega} \bigvee_{n \geq k} x_{n}$, i.e. $\lim \sup x \circ f \leq$ limsup $x$.

(b) follows directly form (a), since each strictly increasing function is an injection.

(c) Since $f$ and $f^{-1}$ are injections, by (b), we have $\lim \sup x \geq \limsup x \circ f \geq$ $\lim \sup x \circ f \circ f^{-1}=\lim \sup x$.

(d) Since $f$ is finite to one, the set $A_{k}=f^{-1}[\{1,2, \ldots, k-1\}]$ is finite for each $k$. Using the same argumentation as in (b), we conclude this proof.

(e) $\limsup x \circ f=\bigwedge_{k \in \omega} \bigvee_{n \geq k} x_{f(n)}=\bigwedge_{k \in \omega} \bigvee_{n \geq k} x_{n+m}=\bigwedge_{k \in \omega \backslash m} \bigvee_{n \geq k} x_{n}$ $\geq \bigwedge_{k \in \omega} \bigvee_{n \geq k} x_{n}=\limsup x$. Since $f$ is an injection, the inverse inequality follows from (b). 
Corollary 3.3.7 If $y$ is a subsequence of a sequence $x$ then $\lim$ inf $x \leq \liminf y$ $\leq \lim \sup y \leq \lim \sup x$.

Lemma 3.3.8 If $x$ and $y$ are sequences in $\mathbb{B}$ such that $x_{n} \leq y_{n}, n \in \omega$, then $\lim \sup x \leq \lim \sup y$ and $\lim$ inf $x \leq \lim$ inf $y$.

Proof. Directly follows from the definitions of limsup and lim inf.

Lemma 3.3.9 $\left[5,2.2\right.$.(v)] For sequences $\left\langle x_{n}: n \in \omega\right\rangle$ and $\left\langle y_{n}: n \in \omega\right\rangle$ we have

$$
\lim \sup \left\langle x_{n} \vee y_{n}\right\rangle=\lim \sup \left\langle x_{n}\right\rangle \vee \lim \sup \left\langle y_{n}\right\rangle \text {. }
$$

Lemma 3.3.10 Let $x=\left\langle x_{n}: n \in \omega\right\rangle$ be a sequence in $\mathbb{B}$ such that the set $\left\{x_{n}=\right.$ $n \in \omega\}$ is finite, then

$$
\begin{aligned}
& \limsup x=\bigvee\left\{b: x_{n}=b \text { for infinitely many } n \in \omega\right\}, \\
& \lim \inf x=\bigwedge\left\{b: x_{n}=b \text { for infinitely many } n \in \omega\right\} .
\end{aligned}
$$

Proof. Since the set $\left\{x_{n}: n \in \omega\right\}$ is finite, there exists $m \in \omega$ such that for each $n_{1} \geq m$ there exists $n_{2}>n_{1}$ such that $x_{n_{2}}=x_{n_{1}}$. For $k \geq m$ let $X_{k}=\left\{x_{n}\right.$ : $n \geq k\}$. Let us notice that $X_{m}=\left\{b: x_{n}=b\right.$ for infinitely many $\left.n \in \omega\right\}$ and that $X_{k}=X_{l}$ for each $k, l \geq m$. By this and by Lemma 3.3.6 (e) we have that $\limsup x=\limsup \left\langle x_{n}: n \geq m\right\rangle=\bigwedge_{k \in \omega} \bigvee_{n \geq k} x_{n+m}=\bigwedge_{k \in \omega} \vee X_{k+m}=$ $\bigvee X_{m}$. The proof for lim inf is analogous.

Definition 3.3.11 Let $\left\langle x_{n}: n \in \omega\right\rangle$ be a sequence in a c.B.a. $\mathbb{B}$ and let $A$ be a subset of $\omega$. Then

$$
\limsup \operatorname{suA}_{n \in A} x_{n}=\bigwedge_{k \in \omega} \bigvee_{n \in A \backslash k} x_{n} \text { and } \liminf _{n \in A} x_{n}=\bigvee_{k \in \omega} \bigwedge_{n \in A \backslash k} x_{n}
$$

Definition 3.3.12 A sequence $x \in \mathbb{B}^{w}$ algebraically converges to $a$, in notation $x \rightarrow a$, iff limsup $x=\liminf x=a$.

Theorem 3.3.13 [37], [5] Let $x=\left\langle x_{n}: n \in \omega\right\rangle$ be a sequence in a c.B.a. $\mathbb{B}$ and let $a$ be an element of $\mathbb{B}$. Then the following conditions are equivalent:

(a) $x \rightarrow a$;

(b) $\limsup \left\langle x_{n} \Delta a\right\rangle=0$;

(c) There exists an increasing sequence $\left\langle a_{n}: n \in \omega\right\rangle$ and a decreasing sequence $\left\langle b_{n}: n \in \omega\right\rangle$ such that $a_{n} \leq x_{n} \leq b_{n}, n \in \omega$ and $\bigvee_{n \in \omega} a_{n}=\bigwedge_{n \in \omega} b_{n}=a$ 
Lemma 3.3.14 [37, 2.1-2.9], [5, 2.2] For the algebraic convergence of sequences there holds:

(a) $\langle b\rangle \rightarrow b$; (L1)

(b) If $x \rightarrow b$ and $y \prec x$ then $y \rightarrow b$; (L2)

(c) Each sequence algebraically converges to at most one element;

(d) If $x$ is an increasing sequence, then $x \rightarrow \bigvee_{n \in \omega} x_{n}$ :

(e) If $x$ is a decreasing sequence, then $x \rightarrow \bigwedge_{n \in \omega} x_{n}$;

(f) $x \rightarrow 0 \Leftrightarrow \lim \sup x=0$;

(g) If the elements of a sequence $x$ are pairwise disjoint, then $x \rightarrow 0$;

(h) If $\left\langle x_{n}\right\rangle \rightarrow b$ and $\left\langle y_{n}\right\rangle \rightarrow c$, then $\left\langle x_{n} \wedge y_{n}\right\rangle \rightarrow b \wedge c,\left\langle x_{n} \vee y_{n}\right\rangle \rightarrow b \vee c$, $\left\langle x_{n} \Delta y_{n}\right\rangle \rightarrow b \Delta c$ and $\left\langle x_{n}^{\prime}\right\rangle \rightarrow b^{\prime}$.

For more details about algebraic convergence of sequences in Boolean algebras we refer to [5] and [37].

\subsection{Measure and submeasure}

Definition 3.4.1 Let $\mathbb{B}$ be a Boolean algebra. A function $\mu: \mathbb{B} \rightarrow[0, \infty)$ is a submeasure on $\mathbb{B}$ iff

(i) $\mu(0)=0$

(ii) $a \leq b \Rightarrow \mu(a) \leq \mu(b)$

(iii) $\mu(a \vee b) \leq \mu(a)+\mu(b)$

A submeasure $\mu$ is:

-strictly positive iff

(i') $\mu(a)=0 \Leftrightarrow a=0$

- exhaustive iff

(iv) For each countable family $\left\{a_{n}: n \in \omega\right\}$ of disjoint elements we have $\lim \mu\left(a_{n}\right)=0$

- uniformly exhaustive iff

(iv') For every $\varepsilon>0$ there exists $n \in \omega$ such that there are no $n$ disjoint elements $a_{1}, \ldots, a_{n} \in \mathbb{B}$ such that $\mu\left(a_{i}\right)>\varepsilon, i=1, \ldots, n$.

- a measure iff

(v) $a \wedge b=0 \Rightarrow \mu(a \vee b)=\mu(a)+\mu(b)$

continuous iff

(vi) $\left\langle a_{n}\right\rangle \backslash 0 \Rightarrow \lim \mu\left(a_{n}\right)=0$

- a $\sigma$-additive measure iff $\mu$ is a measure and 
(vii) $\mu\left(\bigvee_{n \in \omega} a_{n}\right)=\sum_{n \in \omega} \mu\left(a_{n}\right)$ for each sequence $\left\langle a_{n}\right\rangle$ of pairwise disjoint elements of $\mathbb{B}$.

- normed iff

(viii) $\mu(1)=1$

Lemma 3.4.2 [5] A measure $\mu$ on a Boolean algebra is continuous iff $\mu$ is $\sigma$ additive.

Definition 3.4.3 A Boolean algebra $\mathbb{B}$ is a measure algebra iff $\mathbb{B}$ is $\sigma$-complete and there exists a $\sigma$-additive, strictly positive and normed measure $\mu$ on $\mathbb{B}$.

It is well known that each measure algebra is a complete algebra and satisfies the countable chain condition. Also, a measure on a measure algebra $\mathbb{B}$ can generate a metric. Precisely,

Theorem 3.4.4 $[37,2.13]$ Let $\mathbb{B}$ be a measure algebra with a measure $\mu$. Then the function $d: \mathbb{B}^{2} \rightarrow[0, \infty)$, defined by:

$$
d(a, b)=\mu(a \triangle b)
$$

is a metric, and $\langle\mathbb{B}, d\rangle$ is a complete metric space. 
One of the endlessly alluring aspects of mathematics is that its thorniest paradoxes have a way of blooming into beautiful theories.

Philip J. Davis

\section{Chapter 4}

\section{Forcing}

This chapter is reserved for forcing. Some basic notions concerning forcing are presented and the effects of forcing on the set $P(\omega)$ are considered. Also, the basic distributivity laws in Boolean algebra are presented.

\subsection{Generic extension}

This section contains some basic facts about Boolean valued models and generic extensions of models of set theory. The results are mainly taken from [26],

Let $V$ be a transitive countable model of ZFC and $\mathbb{B}$ a c.B.a. in model $V$. Within the ground model $V$, the Boolean valued model $V^{\mathbb{B}}$ is recursively defined by

$-V_{0}^{B}=\emptyset$;

- $V_{\alpha+1}^{\mathbb{B}}=\left\{\tau: \tau\right.$ is a function such that $\operatorname{dom}(\tau) \subset V_{\alpha}^{\mathbb{B}}$ and $\left.\operatorname{ran}(\tau) \subset \mathbb{B}\right\}$;

$V_{\gamma}^{\mathbb{B}}=\bigcup_{\alpha<\gamma} V_{\alpha+1}^{\mathbb{B}}$, for a limit ordinal $\gamma$.

Finally

$$
V^{\mathrm{B}}=\bigcup_{\alpha \in O r d} V_{\alpha}^{\text {曹 }}
$$

Elements of $V^{B}$ are called names and the rank of a name $\tau \in V^{B S}$ is defined by

$$
\operatorname{rank}(\tau)=\min \left\{\alpha: \tau \in V_{\alpha}^{\text {B }}\right\} .
$$


Let $\varphi\left(v_{1}, \ldots, v_{n}\right)$ be a formula let $\tau_{1}, \ldots, \tau_{n}$ be names. The Boolean value $\left\|\varphi\left(\tau_{1}, \ldots, \tau_{n}\right)\right\|$ is recursively defined by

- $\|\tau \in \sigma\|=\bigvee_{\theta \in \operatorname{dom}(\sigma)}(\|\tau=\theta\| \wedge \sigma(\theta))$;

- $\|\tau \subset \sigma\|=\bigwedge_{\theta \in \operatorname{dom}(\tau)}(\tau(\theta) \Rightarrow\|\theta \in \sigma\|)$;

. $\|\tau=\sigma\|=\|\tau \subset \sigma\| \wedge\|\sigma \subset \tau\|$;

- $\left\|\psi_{1} \wedge \psi_{2}\right\|=\left\|\psi_{1}\right\| \wedge\left\|\psi_{2}\right\|$

$\cdot\|\neg \psi\|=\|\psi\|^{\prime}$;

- $\left\|\exists x \psi\left(x, \tau_{1}, \ldots, \tau_{n}\right)\right\|=V_{\tau \in V^{8}}\left\|\psi\left(\tau, \tau_{1}, \ldots, \tau_{n}\right)\right\|$.

A sentence $\varphi$ is true in $V^{\mathbb{B}}$ iff $\|\varphi\|=1$. A formula $\varphi\left(v_{1}, \ldots, v_{n}\right)$ is true in $V^{\text {H }}$ iff for each names $\tau_{1}, \ldots, \tau_{n}$ we have $\left\|\varphi\left(\tau_{1}, \ldots, \tau_{n}\right)\right\|=1$.

Fact 4.1.1 a) Each axiom of $\mathrm{ZFC}$ is true in $V^{\mathrm{B}}$;

b) Each theorem of $\mathrm{ZFC}$ is true in $V^{\mathrm{B}}$;

c) $V^{\mathbb{B}}$ is a Boolean valued model of $\mathrm{ZFC}$.

A filter $G \subset \mathbb{B}$ is a generic filter iff it intersects each dense set of $\mathbb{B}$ belonging to $V$. Since $V$ is a countable model of ZFC, the family of dense sets is also countable. Therefore, there exists a generic filter $G$, which, in general, is not an element of the ground model $V$.

The generic extension $\left.V_{\mathrm{B}} \mid G\right]$, an extension of the ground model $V$ by the generic filter $G$, is defined by

$$
V_{\mathrm{B}}[G]=\left\{\tau_{G}: \tau \in V^{\mathrm{B}}\right\},
$$

where $\emptyset_{G}=\emptyset$ and $\tau_{G}=\left\{\sigma_{G}: \exists b \in G\langle\sigma, b\rangle \in \tau\right\}$.

For each set $A \in V_{\mathbb{B}}[G]$ there exists a name $\bar{A} \in V^{\mathrm{B}}$ such that $\bar{A}_{G}=A$. Such name is not unique. For a set $A \in V$ the canonical name is

$$
\check{A}=\{\langle\check{a}, 1\rangle ; a \in A\} .
$$

For example, $\bar{\emptyset}=\emptyset$ and $(\hat{\emptyset})_{G}=\emptyset$. Also $\dot{1}=\{\emptyset\}^{*}=\{\langle\bar{\emptyset}, 1\rangle\}$, so $\tilde{1}_{G}=$ $\{\langle\bar{\emptyset}, 1\rangle\}_{G}=\{\bar{\emptyset}\}_{G}=\{\emptyset\}$. Inductively, one can prove that

$$
\dot{n}=\{\langle\check{m}, 1\rangle: m<n\} .
$$

The properties of generic extensions are controlled by the choice of $\mathbb{B}$ and $G$ and by the forcing relation $\Vdash$. For an element $p \in \mathbb{B}$ and formula $\phi\left(v_{1}, v_{2}, \ldots, v_{n}\right)$ the forcing relation is defined by

$$
\begin{aligned}
p \Vdash \phi\left(\tau_{1}, \tau_{2}, \ldots, \tau_{n}\right) \text { iff for each generic filter } G \text { containing } p \\
\\
\qquad V_{\mathbb{B}}[G] \vDash \phi\left(\left(\tau_{1}\right)_{G},\left(\tau_{2}\right)_{G}, \ldots,\left(\tau_{n}\right)_{G}\right) .
\end{aligned}
$$

$p$ It $\phi$ can be read as " $p$ forces $\phi$ ", and instead of $V_{\mathrm{B}}[G] \vDash \phi$ we can also write $[\phi]^{V_{B}[G]}$. 


\section{Fact 4.1.2}

(a) $b \Vdash t\left(\tau_{1}, \ldots, \tau_{n}\right)$ iff $b \leq\left\|\phi\left(\tau_{1}, \ldots, \tau_{n}\right)\right\|$.

(b) $1 \Vdash \phi \Rightarrow \psi$ iff $\|\phi\| \leq\|\psi\|$.

(c) $\left.V_{\mathrm{B}} \mid G\right] \vDash \phi$ iff $\|\phi\| \in G$. (Forcing Theorem)

(d) $V_{\mathrm{B}}[G] \vDash \phi$ iff $\exists p \in G p \Vdash \phi$.

(e) If $1 \Vdash \exists x \phi(x)$, then $1 \Vdash \phi(\tau)$ for some $\tau \in V^{\mathrm{b}}$ (The Maximum Principle).

Theorem 4.1.3 $V_{\mathbb{B}}[G]$ is the smallest transitive model of the ZFC theory extending $V$ and containing $G$ as an element.

\subsection{Subsets of $\omega$ and sequences}

Each subset of $\omega$ is represented by its characteristic function, which is an element of $2^{\omega}$. Also, each function $f \in 2^{\omega}$ represents by $\sum_{n \in w} f(n) 2^{-(n+1)}$ a member of the set of the real numbers $\mathbb{R}$, called a real. Therefore, subsets of $\omega$ are, in the language of forcing, usually called reals.

If $x=\left\langle x_{n}: n \in \omega\right\rangle$ is a sequence in a c.B.a. $\mathbb{B}$, then $\tau_{x}=\left\{\left\langle\bar{n}, x_{n}\right\rangle: n \in\right.$ $\omega\} \in V^{\mathbb{B}}$ and $1 \Vdash \tau_{x} \subset \tilde{\omega}$. Clearly $\left\|\bar{n} \in \tau_{x}\right\|=x_{n}$, for each $n \in \omega$. Moreover if $r \in P(\omega) \cap V_{\mathbb{B}}[G]$, then $r=\tau_{G}$ for some $\tau \in V^{\mathbb{B}}$ and there is $b \in G$ such that $b \Vdash \tau \subset \breve{\omega}$. If, in addition, $x_{n}=\|\tilde{n} \in \tau\|, n \in \omega$, then $b \Vdash \tau=\tau_{x}$, so each real belonging to $V_{\mathbb{B}}[G]$ can be represented by a name of the form $\tau_{x}$. Such names will be called nice.

A real $r \in[\omega]^{\omega}$ belonging to a generic extension $V_{\mathrm{B}}[G]$ is new iff $r \notin V$.

A real $r \in[\omega]^{\omega} \cap V_{B}[G]$ is a dependent real iff there exists $A \in[\omega]^{\omega} \cap V$ such that $A \subset r$ or $A \subset \omega \backslash r, r$ is an independent real iff it is not dependent. $r$ is supported iff there exists $A \in[\omega]^{\omega} \cap V$ such that $A \subset T$ and it is unsupported iff it is not supported.

A real $r \in[\omega]^{\omega} \cap V_{\mathbb{B}}[G]$ kills a tall ideal $\mathcal{J} \subset P(\omega), \mathcal{J} \in V$, iff $\forall I \in \mathcal{J}$ $|r \cap I|<\aleph_{0}$. Conceming killing tall ideals we have the following lemma.

Lemma 4.2.1 There exists a tall ideal $\mathcal{J}$ on $\omega$ which is killed in each generic extension containing new reals.

Proof. Let $r \subset \omega$ be a new real from $V_{\mathbb{B}}[G]$. Then its characteristic function, $\chi_{r} \in 2^{\omega} \cap V_{\mathbb{B}}[G]$ determines a new branch $B=\left\{\chi_{r} \mid n: n \in \omega\right\}$ in the tree ${ }^{<} 2$ which clearly kills the tall ideal $\mathcal{J}_{S}$ defined in Example 3.2.3. Let $f:\langle\omega 2 \rightarrow \omega$ be a bijection. Then $\mathcal{J}=\left\{f[S]: S \in \mathcal{J}_{S}\right\}$ is a tall ideal on $\omega$. Consequently, the real $f[B] \subset \omega$ kills the tall ideal $\mathcal{J}$.

In the following theorem we will calculate the Boolean values of several properties related to the nice name of a real. 
Theorem 4.2.2 Let $x=\left\langle x_{n}: n \in \omega\right\rangle$ be a sequence in a c.B.a. $\mathbb{B}, \tau_{x}=\left\{\left\langle\check{n}, x_{n}\right\rangle\right.$ : $n \in \omega\}$ the corresponding nice name for a real, and $A$ an infinite subset of $\omega$. If for an element $b \in \mathbb{B}$ we denote $b^{1}=b$ and $b^{0}=b^{\prime}$ then

(a) $\left\|\tau_{x}=\check{\omega}\right\|=\bigwedge_{n \in \omega} x_{n}$;

(b) $\| \tau_{x}$ is cofinite $\|=\bigvee_{k \in \omega} \bigwedge_{n \geq k} x_{n}(=\liminf x)$;

(c) $\| \tau_{x x}$ is old infinite $\|=\bigvee_{B \in[\omega] \omega} \bigwedge_{n \in \omega} x_{n}{ }^{\chi B(n)}$;

(d) $\| \tau_{x}$ is supported $\|=\bigvee_{B \in(\omega)^{\sim}} \bigwedge_{n \in B} x_{n}$;

(e) $\| \tau_{x}$ is dependent $\|=\bigvee_{B \in[\omega] \omega}\left(\bigwedge_{n \in B} x_{n} \vee \bigwedge_{n \in B} x_{n}^{\prime}\right)_{\text {i }}$

(f) $\| \tau_{x}$ is infinite $\|=\bigwedge_{k \in \omega} \bigvee_{k \geq n} x_{n}(=\lim \sup x)$;

(g) $\left\|\tilde{B} C^{*} \tau_{x}\right\|=\bigvee_{k \in \omega} \bigwedge_{n \in B \backslash k} x_{n}\left(=\liminf _{n \in B} x_{n}\right)$;

(h) $\left\|\left|\tau_{x} \cap \bar{B}\right|=\tilde{\omega}\right\|=\bigwedge_{k \in \omega} \bigvee_{n \in B \backslash k} x_{n}\left(=\lim \sup _{n \in B} x_{n}\right)$.

Proof.

(a) $\left\|\tau_{x}=\tilde{\omega}\right\|=\left\|\forall n \in \tilde{\omega} n \in \tau_{x}\right\|=\bigwedge_{n \in \omega}\left\|\check{n} \in \tau_{x}\right\|=\bigwedge_{n \in \omega} x_{n}$.

(b) $\| \tau_{x}$ is cofinite $\|=\| \exists k \in \check{\omega} \forall n \geq k n \in \tau_{x}\left\|=\bigvee_{k \in \omega} \bigwedge_{n \geq k}\right\| \tilde{n} \in \tau_{x} \|=$ $\bigvee_{k \in \omega} \bigwedge_{n \geq k} x_{n}$

(c) $\| \tau_{x}$ is old infinite $\|=\| \exists B \in\left([\omega]^{\omega}\right)^{V^{-}} \tau_{x}=B\left\|=\bigvee_{B \in[\omega] \omega}\right\| \tau_{x}=\check{B} \|=$ $\bigvee_{B \in[\omega] \omega}\left\|\forall n \in \bar{\omega} n \in \tau_{x} \Leftrightarrow n \in \tilde{B}\right\|=\bigvee_{B \in[\omega] \omega} \bigwedge_{n \in \omega}\left\|\check{n} \in \tau_{x} \Leftrightarrow \check{n} \in \check{B}\right\|=$ $\bigvee_{B \in[\omega] \omega} \bigwedge_{n \in \omega}\left(\left\|\tilde{n} \in \tau_{x} \wedge \dot{n} \in \check{B}\right\| \vee\left\|\tilde{n} \notin \tau_{x} \wedge \check{n} \notin \check{B}\right\|\right)=\left(\bigvee_{B \in[\omega] \omega} \bigwedge_{n \in B} x_{n}\right)$ $\vee\left(\bigvee_{B \in[\omega] \omega} \bigwedge_{n \in \omega \backslash B} x_{n}^{\prime}\right)=\bigvee_{B \in[\omega] \omega} \bigwedge_{n \in \omega} x_{n}^{\chi B(n)}$.

(d) $\| \tau_{x}$ is supported $\|=\| \exists B \in\left([\omega]^{\omega}\right)^{V^{*}} B \subset \tau_{x}\left\|=\bigvee_{B \in[\omega] \omega}\right\| \tilde{B} \subset \tau_{x} \|=$ $\bigvee_{B \in[\omega] \omega}\left\|\forall n \in \tilde{B} n \in \tau_{x}\right\|=\bigvee_{B \in[\omega] \omega} \bigwedge_{n \in B}\left\|\tilde{n} \in \tau_{x}\right\|=\bigvee_{B \in[\omega] \omega} \bigwedge_{n \in B} x_{n}$.

(e) $\| \tau_{x}$ is dependent $\|=\| \exists B \in\left([\omega]^{\omega}\right)^{V^{*}} B \subset \tau_{x} \vee B \subset \dot{\omega} \backslash \tau_{x} \|=\bigvee_{B \in[\omega] \omega}$ $\left\|\check{B} \subset \tau_{x} \vee \bar{B} \subset \breve{\omega} \backslash \tau_{x}\right\|=\bigvee_{B \in[\omega]}\left\|\forall n \in \check{B} n \in \tau_{x}\right\| \vee\left\|\forall n \in \dot{B} n \notin \tau_{x}\right\|=$ $\bigvee_{B \in[\omega]}\left(\bigwedge_{n \in B} x_{n} \vee \bigwedge_{n \in B} x_{n}^{\prime}\right)$.

(f) $\| \tau_{x}$ is infinite $\|=\| \forall k \in \dot{\omega} \exists n \geq k n \in \tau_{x} \|=\bigwedge_{k \in \omega} \bigvee_{k \geq n} x_{n}$.

(g) $\left\|\check{B} \subset^{*} \tau_{x}\right\|=\left\|\exists k \in \breve{\omega} \forall n \in \breve{B} \backslash k n \in \tau_{x}\right\|=\bigvee_{k \in \omega} \bigwedge_{n \in B \backslash k} x_{n}$.

(h) $\left\|\left|\tau_{x} \cap \check{B}\right|=\bar{\omega}\right\|=\left\|\forall k \in \check{\omega} \exists n \geq k n \in \tau_{x} \wedge n \in \check{B}\right\|=\bigwedge_{k \in \omega} \| \exists n \in$ $(B \backslash k) n \in \tau_{x} \|=\bigwedge_{k \in \omega} \bigvee_{n \in B \backslash k} x_{n}$.

Lemma 4.2.3 For an arbitrary sequence $x$ and an infinite $A \subset \omega$ we have

$$
\left\|\tau_{x}=\tilde{\omega}\right\| \leq \| \tau_{x} \text { is cofinite }\|\leq\| \tau_{x} \text { is old infinite } \| \leq
$$

$\leq \| \tau_{x}$ is supported $\|\leq\| \tau_{x}$ is infinite dependent $\|\leq\| \tau_{x}$ is infinite $\| ;$

$\| \tau_{x}$ is cofinite $\|\leq\| \check{A} C^{*} \tau_{x}\|\leq\|\left|\tau_{x} \cap \check{A}\right|=\breve{\omega}\|\leq\| \tau_{x}$ is infinite $\|$. 
Proof. The first sequence of inequalities follows from the sequence of implications

$$
A=\omega \Rightarrow A \text { is cofinite } \Rightarrow A \text { is old infinite } \Rightarrow
$$

$\Rightarrow A$ is supported $\Rightarrow A$ is infinite dependent $\Rightarrow A$ is infinite.

The second sequence of inequalities follows from Lemma 3.3.5.

For each $y \prec x$ there exists $f \in \omega^{\top}$ such that $y=x \circ f$. Can we express $\lim \sup y, \limsup y$ and the nice name $\tau_{y}$, by $\tau_{x}$ and $f$ ? The answer is in the following lemma.

Lemma 4.2.4 If $x$ is a sequence in $\mathbb{B}$ and $f \in \omega^{\text {tw }}$ then

(a) $1 \Vdash \tau_{x \circ f}=\vec{f}^{-1}\left[\tau_{x}\right]$.

(b) $\lim \sup x \circ f=\left\|\left|f[\omega]^{\circ} \cap \tau_{x}\right|=\check{\omega}\right\|$.

(c) $\liminf x \circ f=\left\|f[\omega]^{-} C^{*} \tau_{x}\right\|$.

(d) $1 \Vdash \tau_{x^{\prime}}=\omega \backslash \tau_{x}$, where $x^{\prime}=\left\langle x_{n}^{\prime}: n \in \omega\right\rangle$.

Proof. (a) Suppose $G$ is a $\mathbb{B}$-generic filter over $V$. Then $n \in\left(\tau_{x \circ f}\right)_{G}$ iff $x_{f(n)} \in G$ iff $f(n) \in\left(\tau_{x}\right)_{G}$ iff $n \in f^{-1}\left[\left(\tau_{x}\right)_{G}\right]$, which completes this part of the proof.

(b) Using (a) and Theorem 4.2.2(f), since $f$ is an injection, we obtain that $\limsup x \circ f=\left\|\left|\tau_{x \circ f}\right|=\breve{\omega}\right\|=\left\|\left|\tilde{f}^{-1}\left[\tau_{x}\right]\right|=\breve{\omega}\right\|=\left\|\left|f[\omega]^{*} \cap \tau_{x}\right|=\tilde{\omega}\right\|$.

(c) Similar to the proof of (b).

(d) Follows from the fact that $n \in\left(\tau_{x^{\prime}}\right)_{G}$ iff $x_{n}^{\prime} \in G$ iff $x_{n} \notin G$ iff $n \in$ $\omega \backslash\left(\tau_{x}\right)_{G}$.

For a sequence $x$ in a c.B.a. $\mathbb{B}$ we introduce two elements $a_{x}$ and $b_{x}$.

Definition 4.2.5 Let $\mathbb{B}$ be a c.B.a. If $x$ is a sequence in $\mathbb{B}$, then let

$$
\begin{aligned}
& a_{x}=\bigwedge_{A \in[\omega] \omega} \bigvee_{B \in[A]^{\omega}} \bigvee_{k \in \omega} \bigwedge_{n \in B \backslash k} x_{n} \\
& b_{x}=\bigvee_{A \in[\omega]} \bigwedge_{B \in[A]^{\omega}} \bigwedge_{k \in \omega} \bigvee_{n \in B \backslash k} x_{n}
\end{aligned}
$$

Lemma 4.2.6 Let $x$ be a sequence in a c.B.a $\mathbb{B}$. Then

$$
\begin{aligned}
& a_{x}=\bigwedge_{A \in[\omega] \omega} \bigvee_{B \in[A]} \liminf \inf _{n \in B} x_{n} \\
& =\bigwedge_{\lambda \in[\omega] \omega} \bigvee_{B \in[A]^{\omega}}\left\|\tilde{B} C^{*} \tau_{x}\right\| \\
& \left.=\| \forall A \in\left((\mid \omega]^{\omega}\right)^{V}\right) \exists B \in\left(\left([A]^{\omega}\right)^{V}\right) B C^{*} \tau_{x} \| \\
& =\left\|\forall A \in\left(\left([\omega]^{\omega}\right)^{V}\right) \exists B \in\left(\left([A]^{\omega}\right)^{V}\right)^{\top} B \subset \tau_{x}\right\| \\
& =\bigwedge_{A \in[\omega]^{\omega}} \bigvee_{B \in[A]^{\omega}} \bigwedge_{n \in B} x_{n} ; \\
& b_{x}=\bigvee_{A \in[\omega]^{\omega}} \bigwedge_{B \in[A]^{\omega}} \lim \sup _{n \in B} x_{n}
\end{aligned}
$$




$$
\begin{aligned}
& =\bigvee_{A \in[\omega]} \bigwedge_{B \in[A]^{\omega}}\left\|\left|\tau_{x} \cap \hat{B}\right|=\tilde{\omega}\right\| \\
& =\left\|\exists A \in\left(\left([\omega]^{\omega}\right)^{V}\right) \forall B \in\left(\left([A]^{\omega}\right)^{V}\right)-\tau_{x} \cap B \mid=\tilde{\omega}\right\| \\
& =\left\|\exists A \in\left(\left([\omega]^{\omega}\right)^{V}\right) \forall B \in\left(\left([A]^{\omega}\right)^{V}\right) \tau_{x} \cap B \neq \tilde{\varphi}\right\| \\
& =\bigvee_{A \in[\omega]} \bigwedge_{B \in[A]^{\omega}} \bigvee_{n \in B} x_{n-}
\end{aligned}
$$

Lemma 4.2.7 For a sequence $x \in \mathbb{B}$ we have

$$
\liminf x \leq a_{x} \leq \| \tau_{x} \text { is supported } \| \leq b_{x} \leq \lim \sup x .
$$

Proof. The only thing which is not obvious is $\| \tau_{x}$ is supported $\| \leq b_{x}$, i.e.

$$
\bigvee_{B \in[\omega] \omega} \bigwedge_{n \in B} x_{n} \leq \bigvee_{B \in[\omega] \omega} \bigwedge_{A \in[B] \omega} \bigvee_{n \in A} x_{n}
$$

But, this follows directly from the statement

$$
\forall A \in[B]^{\omega} \exists n \in B x_{n} \leq \bigvee_{n \in A} x_{n}
$$

where $B$ is an infinite subset of $\omega$.

\subsection{Mappings on $\omega$ and matrices}

Definition 4.3.1 A c.B.a. $\mathbb{B}$ is $(\kappa, \lambda)$-distributive iff for any matrix $\left[a_{\alpha \beta}: \alpha \epsilon\right.$ $\kappa, \beta \in \lambda]$ in $\mathbb{B}$ there holds

$$
\bigwedge_{\alpha \in \kappa} \bigvee_{\beta \in \lambda} a_{\alpha \beta}=\bigvee_{f \in \lambda^{*}} \bigwedge_{\alpha \in \kappa} a_{\alpha f(\alpha)}
$$

$\mathbb{B}$ is $(\kappa, \infty)$-distributive iff it is $(\kappa, \lambda)$-distributive for every $\lambda$, and $\mathbb{B}$ is completely distributive iff it is $(\kappa, \lambda)$-distributive for each $\kappa$ and $\lambda$.

If $\mathbb{B}$ is $(\kappa, \lambda)$-distributive, then it is $\left(\kappa^{\prime}, \lambda^{\prime}\right)$-distributive, for each $\kappa^{\prime} \leq \kappa$ and $\lambda^{\prime} \leq \lambda$.

For finite $\kappa$ and $\lambda$ distributive laws are satisfied. Also, each Boolean algebra is $(\kappa, 1)$-distributive for each cardinal $\kappa$.

Remark 4.3.2 A complete Boolean algebra $\mathbb{B}$ is $(\kappa, \lambda)$-distributive iff in each generic extension each function from $\kappa$ to $\lambda$ belongs to the ground model.

A complete Boolean algebra $\mathbb{B}$ is $(\omega, 2)$-distributive iff $\mathbb{B}$ does not add new reals by forcing. 
Definition 4.3.3 A Boolean algebra $\mathbb{B}$ is $(\omega, \kappa)$-weakly distributive iff for each matrix $\left[a_{n \alpha}: n \in \omega, \alpha \in \kappa\right]$ in $\mathbb{B}$ we have

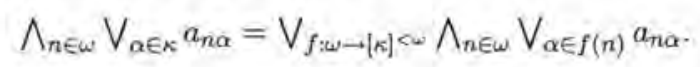

A Boolean algebra $\mathbb{B}$ is called weakly distributive iff it is $(\omega, \omega)$-weakly distributive.

Namba [42] has shown the consistency of the equivalence between $\left(\omega, \omega_{1}\right)$ weak distributivity and weak distributivity, and also the consistency that these two conditions are not equivalent.

Now we give two technical lemmas about matrices in c.B.a.'s and names of functions from $\omega$ into $\omega$.

Lemma 4.3.4 Let $b \Vdash \tau \subset\left(\omega^{2}\right)$. Then

$b \Vdash \tau: \breve{\omega} \rightarrow \breve{\omega} \Leftrightarrow \forall n \in \omega\{\|\langle n, k\rangle \in \tau\| \wedge b: k \in \omega\}$ is a quasi-partition of $b$.

Proof. First, let us notice that

$$
\begin{aligned}
\|\tau: \dot{\omega} \rightarrow \tilde{\omega}\| & =\|\forall n \in \tilde{\omega} \exists k \in \tilde{\omega}\langle n, k\rangle \in \tau\| \\
& \wedge\|\forall n \in \tilde{\omega} \forall k \in \tilde{\omega} \forall l>k \neg(\langle n, k\rangle \in \tau \wedge\langle n, l\rangle \in \tau)\| .
\end{aligned}
$$

$\Leftrightarrow$ The inequality $b \leq\|\tau: \dot{\omega} \rightarrow \dot{\omega}\|$ (which is equivalent to $b \Vdash \tau: \dot{\omega} \rightarrow \breve{\omega}$ ) implies $b \leq \bigwedge_{n \in \omega} \bigvee_{k \in \omega}\left\|\langle n, k\rangle^{-} \in \tau\right\|$. Therefore, for each $n \in \omega$ we have $b=b \wedge \bigvee_{k \in \omega}\left\|\langle n, k\rangle^{-} \in \tau\right\|$, so

$$
b=\bigvee_{k \in \omega}\left(\left\|\langle n, k\rangle^{-} \in \tau\right\| \wedge b\right) .
$$

Also, we have

$$
b \leq \bigwedge_{n \in \omega} \bigwedge_{k \in \omega} \bigwedge_{l>k}\left(\left\|\langle n, k\rangle^{-} \in \tau\right\| \wedge\left\|\langle n, l\rangle^{-} \in \tau\right\|\right)^{\prime},
$$

so for each $n, k \in \omega$ and $l>k$ there holds

$$
b \leq\left(\left\|\langle n, k\rangle^{-} \in \tau\right\| \wedge\left\|\langle n, l\rangle^{-} \in \tau\right\|\right)^{\prime},
$$

i.e.

$$
b \wedge\left(\left\|\langle n, k\rangle^{-} \in \tau\right\| \wedge\left\|\langle n, l\rangle^{-} \in \tau\right\|\right)=0 .
$$

Therefore

$$
\left(\left\|\langle n, k\rangle^{-} \in \tau\right\| \wedge b\right) \wedge\left(\left\|\langle n, l\rangle^{-} \in \tau\right\| \wedge b\right)=0, k \neq l
$$


$(\Leftarrow)$ For each $n \in \omega$ let the family $\{\|\langle n, k\rangle \in \tau\| \wedge b: k \in \omega\}$ be a quasipartition of $b$. Let us prove that $b \Vdash \tau: \tilde{\omega} \rightarrow \bar{\omega}$. Let $b \in G$ where $G$ is a generic filter in $\mathbb{B}$ and let $n \in \omega$. The family $\{\|\langle n, k\rangle \in \tau\| \wedge b: k \in \omega\}$ satisfies (4.3) and (4.4), which implies that

$$
\left\{\left\|\langle n, k\rangle^{\swarrow} \in \tau\right\| \wedge b: k \in \omega\right\} \backslash\{0\}
$$

is a maximal antichain below $b$. So, there exists a unique $k \in \omega$ such that $\|\langle n, k\rangle^{-} \in$ $\tau \| \wedge b \in G$, which gives us that $\left\|\langle n, k\rangle^{-} \in \tau\right\| \in G$, i.e. $\langle n, k\rangle \in \tau_{G}$. Hence, $\forall n \in \omega \exists_{1} k \in \omega\langle n, k\rangle \in \tau_{G}$, which implies that $\tau_{G} \cap \omega^{2}: \omega \rightarrow \omega$. But, since $b \Vdash \tau \subset\left(\omega^{2}\right)$, we have $\tau_{G}: \omega \rightarrow \omega$.

Lemma 4.3.5 Let $\varphi$ be a formula of ZFC and

$$
b \Vdash \tau: \bar{\omega} \rightarrow \bar{\omega} \wedge \tau \text { is increasing } \wedge \varphi(\tau) .
$$

Then there exists a very nice name $\sigma$ for a subset of $\omega^{2}$, such that

(a) $1 \Vdash \sigma: \bar{\omega} \rightarrow \bar{\omega} \wedge \sigma$ is increasing:

(b) $b \Vdash \sigma: \bar{\omega} \rightarrow \breve{\omega} \wedge \sigma$ is increasing $\wedge \varphi(\sigma)$.

Proof. Let (4.5) hold, and let us define $\sigma=\left\{\left\langle\langle n, k\rangle^{*}, c_{n, k}\right\rangle: n, k \in \omega\right\}$, where

$$
c_{n, k}= \begin{cases}\left\|\langle n, k\rangle^{-} \in \tau\right\| \wedge b & n \neq k \\ \left\|\langle n, k\rangle^{-} \in \tau\right\| \vee b^{\prime} & n=k\end{cases}
$$

(a) Let $G$ be a generic filter in $\mathbb{B}$. Then we have two possibilities:

$1^{\circ} b \in G$. Let us prove that

$$
\sigma_{G}=\tau_{G}
$$

(C) Since $\operatorname{dom} \sigma=\omega^{2}$, we have that $\sigma_{G} \subset \omega^{2}$. Let $\langle n, k\rangle \in \sigma_{G}$. Then $\langle n, k\rangle_{G} \in \sigma_{G}=\left\{\pi_{G}: \exists q \in G\left\langle\pi_{1} q\right\rangle \in \sigma\right\}$, so there exists $q \in G$ such that $\langle\langle n, k\rangle ; q\rangle \in \sigma$, which implies $q=c_{n, k} \in G$. If $n \neq k$, then $\left\|\langle n, k\rangle^{-} \in \tau\right\| \wedge b \in G$. which gives us $\left\|\langle n, k\rangle^{-} \in \tau\right\| \in G$, i.e. $\langle n, k\rangle \in \tau_{G}$. If $n=k$ then $\|\langle n, k\rangle^{-} \in$ $\tau \| \vee b^{\prime} \in G$. From $b \in G$ it follows that $\|\langle n, k\rangle-\in \tau\| \wedge b \in G$, which, again, gives $\langle n, k\rangle \in \tau_{G}$.

(つ) From $b \Vdash \tau: \bar{\omega} \rightarrow \bar{\omega}$ and $b \in G$ it follows that $\tau_{G} \subset \omega^{2}$. Let $\langle n, k\rangle \in \tau_{G}$. Then $\left\|\langle n, k\rangle^{-} \in \tau\right\| \in G$, and also $\left\|\langle n, k\rangle^{-} \in \tau\right\| \wedge b \in G$. We have that $c_{n, k} \geq$ $\left\|\langle n, k\rangle^{*} \in \tau\right\| \wedge b$, so $c_{n, k} \in G$, and, therefore, $\langle n, k\rangle \in \sigma_{G}$.

As $b \in G$, from (4.5) it follows that $\tau_{G}: \omega \rightarrow \omega$ is an increasing function. Now, (4.6) implies

$$
\sigma_{G}: \omega \rightarrow \omega \wedge \sigma_{G} \text { is an increasing function. }
$$


$2^{\circ} b^{\prime} \in G$. Let us prove that

$$
\sigma_{G}=i d_{\omega}
$$

(c) Let $\langle n, k\rangle \in \sigma_{G}$. Then also $c_{n, k} \in G$. From $n \neq k$ it would follow that $b \in G$, which is impossible. Therefore $n=k$, and $\langle n, k\rangle \in i d_{w}$.

(つ) Let $\langle n, n\rangle \in i d_{\omega}$. Since $b^{\prime} \in G$, we have $c_{n, n}=\left\|\langle n, n\rangle^{-} \in \tau\right\| \vee b^{\prime} \in G$, and therefore $\langle n, n\rangle \in \sigma_{G}$.

Since $i d_{\omega}: \omega \rightarrow \omega$ is an increasing function, we have proved (a).

(b) In (a) it is showen that $b \Vdash \sigma=\tau$. From that and (4.5) we obtain (b).

Theorem 4.3.6 $[5, \mathrm{p} .7]$ For a Boolean algebra $\mathbb{B}$ the following conditions are equivalent:

(a) $\mathbb{B}$ is weakly distributive;

(b) $\mathbb{B}$ is $\omega^{\omega}$-bounding, i.e. $1 \Vdash \forall g \in \omega^{\omega} \exists f \in\left(\omega^{\omega} \cap V\right)^{\circ} g \leq f$;

(c) For each increasing matrix $\left[a_{n k}\right]$ there holds $\bigvee_{f \in \omega^{\omega}} \liminf a_{n f(n)}=1$;

(d) For each decreasing matrix $\left[b_{n k}\right]$ there holds $\bigwedge_{f \in \omega^{w}} \limsup b_{n f(n)}=0$.

Proof. (a) $\Leftrightarrow$ (b). According to [26, p. 264], $\mathbb{B}$ is weakly distributive iff $\mathbb{B}$ is an $\omega^{\omega}$-bounding.

(a) $\Rightarrow$ (c). Let $\mathbb{B}$ be a weakly distributive Boolean algebra and let $\left[a_{n k}\right]$ be an increasing matrix in $\mathbb{B}$. So, $\bigwedge_{n \in \omega} \bigvee_{k \in \omega} a_{n k}=\bigwedge_{n \in \omega} 1=1$. From (4.2) it follows that

$$
\bigvee_{f i \omega \rightarrow[\omega]<\omega} \bigwedge_{n \in \omega} \bigvee_{\alpha \in f(n)} a_{n \alpha}=1
$$

Let $f: \omega \rightarrow[\omega]^{<\omega}$ and $n \in \omega$. Then $f(n)$ is a finite subset of $\omega$. Since, the matrix $\left[a_{n k}\right]$ is increasing, we have $\bigvee_{a \in f(n)} a_{n \alpha}=a_{n, \max f(n)}$. So,

$$
V_{f: \omega \rightarrow|\omega|<\omega} \bigwedge_{n \in \omega} a_{n, \max f(n)}=1 .
$$

Let us prove

$$
\bigvee_{g \in \omega^{\omega}} \bigwedge_{n \in \omega^{\omega}} a_{n g(n)}=1
$$

Let $\alpha>0$. Equality (4.10) implies that there exists a function $f \in \omega^{\omega}$ such that $\bigwedge_{n \in \omega} a_{n, \max f(n)} \wedge a>0$. Let $g \in \omega^{\omega}$ be the function defined by $g(n)=$ $\max f(n)$. Then $\bigwedge_{n \in \omega} a_{n g(n)} \wedge a>0$, and also

$$
\left(\bigvee_{g \in \omega} \bigwedge_{n \in \omega} a_{n g(n)}\right) \wedge a>0 .
$$

From this it follows (4.11), since, otherwise, for $a=\left(\bigvee_{g \in \omega^{\omega}} \bigwedge_{n \in \omega} a_{n g(n)}\right)^{\prime}$ equality (4.12) is not true. 
For each function $g \in \omega^{\omega}$ we have $\liminf a_{n g(n)} \geq \bigwedge_{n \in \omega} a_{n g(n)}$. From this and (4.11) it follows

$$
\bigvee_{g \in \omega^{\omega}} \liminf a_{n g(n)} \geq 1
$$

(c) $\Rightarrow$ (b). One can verify that a Boolean algebra $\mathbb{B}$ is $\omega^{\omega}$-bounding iff

$$
\text { I If } \forall x \in \omega^{\omega} \exists f \in\left(\omega^{\omega} \cap V\right)^{2} x \leq^{*} f \text {. }
$$

Let $\tau$ be a nice name for a function which maps $\omega$ into $\omega$, i.e. $\tau=\left\{\left\langle\langle n, k\rangle, a_{n k}\right\rangle\right.$ : $n, k \in \omega\}$ and $1 \Vdash \tau: \check{\omega} \rightarrow \bar{\omega}$. Then the sets $\left\{a_{n k}: k \in \omega\right\}, n \in \omega$, by Lemma 4.3.4, are quasi-partitions of the unity. Let us define $b_{n k}=\bigvee_{i \leq k} a_{n i}, k, n \in \omega$. The matrix $\left[b_{n k}\right]$ is increasing, so

$$
V_{f \in \omega^{w}} \liminf b_{n f(n)}=1 \text {. }
$$

There holds

$$
\begin{aligned}
\bigvee_{f \in \omega^{\omega}} \liminf b_{n f(n)} & =\bigvee_{f \in \omega^{\omega}} \bigvee_{m \in \omega} \bigwedge_{n \geq m} \bigvee_{i \leq f(n)} a_{n i} \\
& =\left\|\exists f \in\left(\omega^{\omega} \cap V\right)^{*} \exists m \in \tilde{\omega} \forall n \geq m \exists i \leq f(n) \tau(n)=i\right\| \\
& =\left\|\exists f \in\left(\omega^{\omega} \cap V\right)^{-} \exists m \in \tilde{\omega} \forall n \geq m \tau(n) \leq f(n)\right\| \\
& =\left\|\exists f \in\left(\omega^{\omega} \cap V\right)^{-} \tau \leq^{*} f\right\| .
\end{aligned}
$$

Therefore, and from (4.14) it follows

$$
\left\|\exists f \in\left(\omega^{\omega} \cap V\right)^{*} \tau \leq^{*} f\right\|=1,
$$

which completes this part of the proof.

(c) $\Rightarrow(\mathrm{d})$. Let $\left[b_{n k}\right]$ be a decreasing matrix. Then, according to Lemma 3.3.3, the matrix $\left[b_{n k}^{\prime}\right]$ is increasing. So, (c) implies that

$$
\bigvee_{f \in w^{\alpha}} \bigvee_{k \in \omega} \bigwedge_{n \geq k} b_{n f(n)}^{\prime}=1
$$

so

$$
\bigwedge_{f \in \omega^{\omega}} \bigwedge_{k \in \omega} \bigvee_{n \geq k} b_{n f(n)}=0,
$$

which is equivalent to

$$
\bigwedge_{f \in \omega^{\omega}} \limsup b_{n f(n)}=0 .
$$

(d) $\Rightarrow$ (c). Analogously. 
Theorem 4.3.7 [5, Lemma 3.5.] For a Boolean algebra $\mathbb{B}$ the following conditions are equivalent:

(a) $\mathbb{B}$ is weakly-distributive and $\mathrm{b}-c c$;

(b) For each increasing matrix $\left[a_{n k}\right]$ there exists $g \in \omega^{\omega}$ such that $\liminf a_{n g(n)}=1$;

(c) For each decreasing matrix $\left[b_{n k}\right]$ there exists $g \in w^{i}$ such that $\limsup b_{n g(n)}=0$.

Proof. $(\mathrm{a}) \Rightarrow$ (b). Let $\mathbb{B}$ be a weakly distributive b-cc Boolean algebra and let $\left[a_{n k}\right]$ be an increasing matrix. According to Theorem 4.3.6 we have

$$
\bigvee_{f \in \omega^{\omega}} \liminf a_{n, f(n)}=1 \text {. }
$$

Since $\mathbb{B}$ is $b$-cc, there exists a family $\varphi \subset \omega^{\omega}$ of size less then $b$ such that

$$
V_{f \in \varphi} \liminf a_{n f(n)}=1
$$

Also, there exists a function $g \in \omega^{\omega}$ such that for all $f \in \varphi$ we have $f \leq g$. Since $\left[a_{n k}\right]$ is an increasing matrix, for all $f \in \varphi$ we have that $\liminf a_{n, f(n)} \leq$ liminf $a_{n, g(n)}$, and, therefore

$$
\lim \inf a_{n g(n)}=1 .
$$

(b) $\Rightarrow$ (a). Let (b) hold. Then, by Theorem 4.3.6, $\mathbb{B}$ is weakly distributive. Let us prove that $\mathbb{B}$ is $b$-cc. To prove this, we will show that for each $A \subset \mathbb{B}$, where $A$ is a partition of the unity, we have $|A|<b$. Let $\left\{f_{a} \in w^{\omega}: a \in A\right\}$ be a family of functions, and let

$$
a_{n k}=\bigvee\left\{a \in A: f_{a}(n)<k\right\} .
$$

One can easily verify that the matrix $\left[a_{n k}\right]$ is increasing. Therefore, there exists a function $g \in \omega^{\omega}$ such that

$$
\liminf a_{n g(n)}=\bigvee \bigwedge_{m \in \omega} a_{n \geq m} a_{n g(n)}=1 .
$$

Since $A$ is an antichain, for each $a \in A$ there exists an $m_{a} \in \omega$ such that $\bigwedge_{n \geq m_{a}} a_{n g(n)} \geq a$, which implies that $a_{n g(n)} \geq a$, for each $n \geq m_{a}$. Since

$$
a_{n g(n)}=\bigvee\left\{a \in A: f_{a}(n)<g(n)\right\},
$$

we conclude that $f_{a}(n)<g(n)$ for each $n \geq m_{a}$. Therefore, the family $\left\{f_{a} \in\right.$ $\left.\omega^{\omega}: a \in A\right\}$ is bounded by $g$. Since we can bound each family of functions of size $|A|$, we conclude that the size of each antichain is less then $b$.

(b) $\Leftrightarrow(c)$. The proof is analogous to the proof $(c) \Leftarrow$ (d) of Theorem 4.3.6. 
Remark 4.3.8 The regular-open algebra of the Sacks forcing (see [44]) is an example of a Boolean algebra which is weakly-distributive, but not b-cc. Cohen forcing (see [14]) is b-cc but not weakly-distributive. Random forcing (see [33]) is weakly-distributive and $b$-cc. 


\section{Part II}

\section{Topologies on complete Boolean algebras}



In mathematics, you don't understand things.

You just get used to them.

John von Neumann

\section{Chapter 5}

\section{The sequential topology on complete Boolean algebras}

This chapter is reserved for the sequential topology $\tau_{s}$ on complete Boolean algebras. Section 5.1 contains known results related to $\tau_{s}$. In Section 5.2 the class of lim sup-stable algebras and condition $(\hbar)$ is defined, which are more investigated in Section 5.3. Section 5.4 is reserved for new results about topological limit in Boolean algebras with the sequential topology. In Section 5.5 the class of sequentially compact spaces with the sequential topology is characterized. It is proved that the space $\left\langle\mathbb{B}, \tau_{s}\right\rangle$ is sequentially compact iff $\mathbb{B}$ is a lim sup-stable algebra and forcing by $\mathbb{B}$ does not produce independent reals.

\subsection{Basic properties}

Let $\mathbb{B}$ be a complete Boolean algebra and $x$ a sequence in $\mathbb{B}$. The operator of the algebraic convergence $\lambda_{A}$ (see Definition 3.3.12) is defined by

$$
\lambda_{A}(x)=\left\{\begin{array}{cc}
\{\limsup x\} & \text { if } \liminf x=\limsup x \\
\emptyset & \text { if } \liminf x<\operatorname{linsup} x
\end{array}\right.
$$

According to Theorem 4.2.2 there holds 


$$
\lambda_{A}(x)=\left\{\begin{array}{cl}
\left\{\| \tau_{x} \text { is infinite } \|\right\} & \text { if } \| \tau_{x} \text { is cofinite }\|=\| \tau_{x} \text { is infinite } \| \\
\emptyset & \text { if } \| \tau_{x} \text { is cofinite }\|<\| \tau_{x} \text { is infinite } \|
\end{array}\right.
$$

The topology generated by $\lambda_{A}$ as an a priori limit operator is known as the sequential topology on complete Boolean algebras and denoted by $\tau_{s}$. This topology is closely related to von Neuman's problem [38], since Dorothy Maharam in 1947 [37] showed that the existence of the Maharam submeasure on $\mathbb{B}$ is equivalent to metrizability of the space $\left\langle\mathbb{B}, \tau_{s}\right\rangle$.

The results given in this section are amalgamation of results presented in papers [5], [6], [7] and [37]. Most of them will be given without proof.

By Lemma 3.3.14, the a priori limit operator $\lambda_{A}$ satisfies conditions (L1) and (L2) and $\lambda_{A}$-limit is an empty set or a singleton. These conditions are still not enough to ensure the equality between the a priori and the a posteriori convergence. Concerning the a posteriori convergence we have the following.

Theorem 5.1.1 $[5, \mathrm{p} .4]$ The sequence $x$ converges a posteriori to the point $a$ iff each subsequence of $x$ has a subsequence that converges to $a$ a priori.

By Theorem 2.3.9, $\lambda_{A}^{*}$, the closure of a priori operator $\lambda_{A}$ under (L3) has also the unique limit. By a known result of Kisyniski (see [32] and [16, 1.7.19]), the a posteriori limit operator coincides with $\lambda_{A}^{*}$ and it is an empty set or a singleton. Therefore, instead $\lim _{\tau_{a}} x=\{b\}$ we will briefly write $\lim x=b$ or $x \rightarrow \tau_{3}, b$. From the uniqueness of the topological limit it follows that the singletons are closed sets, and the space $\left\langle\mathbb{B} ; \tau_{s}\right\rangle$ is a $T_{1}$-space.

$\left\langle\mathbb{B}, \tau_{s}\right\rangle$ is a sequential space, but it must not be a Fréchet space and $\left\langle\mathbb{B}, \tau_{s}\right\rangle$ is a Fréchet space iff for the operator $u$ (see Definition 2.3.11) there holds $u=u^{2}$. Moreover, we have the following theorem.

Theorem 5.1.2 [5, Th. 3.4.] The space $\left\langle\mathbb{B}, \tau_{s}\right\rangle$ is a Fréchet space iff $\mathbb{B}$ is weakly distributive and $b-c c$.

If $\mathbb{B}$ is a measure algebra with the measure $\mu$, then, as mentioned in Theorem 3.4.4. $d(a, b)=\mu(a \triangle b)$ is a metric on $\mathbb{B}$, but also the topology generated by the metric $d$ and $\tau_{s}$ coincide.

In the space $\left\langle\mathbb{B}, \tau_{s}\right\rangle$ the operation of complementation is continuous. Also, for a fixed element $a$, the function which maps an element $x$ to $a \wedge x(a \vee x, a \triangle x)$ is

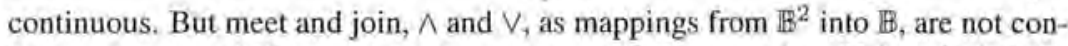
tinuous in general. For any two elements $a$ and $b$, the mapping $f(x)=(x \triangle b) \triangle a$ is an automorphism satisfying $f(a)=b$ and $f(b)=a$. Therefore, the space is 
homogeneous, which implies that the topology $\tau_{\delta}$ is determined by the neighborhoods of 0 , denoted by $\mathcal{N}_{0}$, and if $a \in U \in \tau_{s}$, then $a \Delta U \in \mathcal{N}_{0}$. Therefore, if $\mathbb{B}$ is infinite, there are no isolated points (the space is perfect). Concerning a neighborhood base of 0 , we have following.

Theorem 5.1.3 [5, Prop. 3.1.] If $\left\langle B, \tau_{s}\right\rangle$ is a Fréchet space, then for every $V \in \mathcal{N}_{0}$ there exists $U \subset V$ such that $U \in \mathcal{N}_{0}$ and $U$ is downward closed.

The family of downward closed neighborhoods of 0 is denoted by $\mathcal{N}_{0}^{d}$. If $\mathcal{N}_{0}^{d}$ is a neighborhood base of 0 , then the family $a \Delta \mathcal{N}_{0}^{d}$ is a neighborhood base of the point $a$.

The space $\left\langle\mathbb{B}, \tau_{s}\right\rangle$ is not always a Hausdorff space. In fact, this is rather strong condition.

Theorem 5.1.4 [5, Th. 4.1.] If $\left\langle\mathbb{B}, \tau_{s}\right\rangle$ is a Hausdorff space, then $\mathbb{B}$ is $\left(\omega, \omega_{1}\right)$ weakly distributive, which implies weak distributivity.

Theorem 5.1.5 [5] For each cardinal $\kappa,\left\langle P(\kappa), \tau_{s}\right\rangle$ is a Hausdorff space.

Theorem 5.1.6 $\left[5, \operatorname{Cor}\right.$. 4.8.] If $\left\langle\mathbb{B}, \tau_{s}\right\rangle$ is a regular space, then $\mathbb{B}$ is a ccc algebra.

Let us notice that $P\left(\omega_{1}\right)$ is not a cce algebra, and, by the previous theorem, $\left\langle P\left(\omega_{1}\right), \tau_{s}\right)$ is not a regular space.

Theorem 5.1.7 [5, Th. 5.1.] Let $\mathbb{B}$ be a c.B.a. Then the following conditions are equivalent.

(a) $\mathbb{B}$ is ccc and $\left\langle\mathbb{B}, \tau_{\xi}\right\rangle$ is a Hausdorff space;

(b) There exists a countable family $\left\{U_{n}: n \in \omega\right\} \subset \mathcal{N}_{0}$ such that $\bigcap_{n \in w} \overline{U_{n}}=\{0\}$;

(c) The operation $\vee$ is continuous in $\langle 0,0\rangle$;

(d) $\left\langle\mathbb{B}, \tau_{s}\right\rangle$ is a regular space;

(e) $\left\langle\mathbb{B}, \tau_{s}\right\rangle$ is a metrizable space;

(f) $\mathbb{B}$ caries a strictly positive Maharam submeasure,

Since every Boolean algebra which caries Maharam submeasure is a ccc and a weakly distributive algebra, there holds that each metrizable space of the form $\left\langle\mathbb{B}, \tau_{s}\right\rangle$ is a Fréchet space. The reverse is not true in general, since the Suslin algebra with the sequential topology is a Fréchet space which is not Hausdorff. Also, $\left\langle P(b), \tau_{s}\right\rangle$ is a Hausdorff space which is not Fréchet.

Concerning the Boolean algebra $P(\kappa)$ we have: 
Theorem 5.1.8 [5, Ex. 4.3]

(a) Tychonoff product topology on $P(\kappa)$ (which can be identified with the Cantor cube $2^{\kappa}$ ) is coarser than the sequential topology $\tau_{s}$.

(b) For uncountable $\kappa$, the space $\left\langle P(\kappa), \tau_{s}\right\rangle$ is not compact.

(c) For uncountable $\kappa$, the product topology is strictly coarser than $\tau_{s}$.

(d) $\left\langle P(\omega), \tau_{s}\right\rangle$ is homeomorphic to the Cantor set $2^{\omega}$.

(e) $\left\langle P(\omega)_{+} \tau_{s}\right\rangle$ is a compact space.

\subsection{Condition $(\hbar)$}

Definition 5.2.1 Let $x$ be a sequence in a c.B.a. $\mathbb{B}$. The sequence $x$ is lim supstable iff for each subsequence $y \prec x$ we have $\lim \sup y=\lim \sup x$.

The sequence $x$ is liminf-stable iff for each subsequence $y \prec x$ we have $\liminf y=\liminf x$.

The Boolean algebra $\mathbb{B}$ is lim sup-stable iff each sequence has a lim sup-stable subsequence.

One can easily verify that $\mathbb{B}$ is limsup-stable iff each sequence has a lim infstable subsequence.

Definition 5.2.2 Let $x$ be a sequence in a c.B.a. $\mathbb{B}$. A set $A \in[\omega]^{\omega}$ is $x$-stable iff

$$
\forall B \in[A]^{\omega}\left\|\left|\tau_{x} \cap \bar{B}\right|=\bar{\omega}\right\|=\left\|\left|\tau_{x} \cap \bar{A}\right|=\bar{\omega}\right\|
$$

Definition 5.2.3 A c.B.a. B satisfies condition $(\hbar)$ iff for each sequence $x$ the set

$$
D_{x}=\left\{A \in[\omega]^{\omega}: A \text { is } x \text {-stable }\right\}
$$

is dense in $\langle[\omega] \omega, c\rangle$.

Lemma 5.2.4 The set $D_{x}$ is downward closed in $\left\langle[\omega]^{\omega}, C\right\rangle$.

Proof. Let $A_{1}$ is a $x$-stable set and $A_{2} \subset A_{1}$. We will prove that $A_{2}$ is $x$ stable. Let $B \subset A_{2}$. Then $B \subset A_{1}$ and since $A$ is $x$-stable, $\left\|\left|\tau_{x} \cap \breve{B}\right|=\tilde{\omega}\right\|=$ $\left\|\left|\tau_{x} \cap \check{A}_{1}\right|=\tilde{\omega}\right\|$. From $A_{2} \subset A_{1}$ it follows $\left\|\left|\tau_{x} \cap \tilde{A}_{2}\right|=\tilde{\omega}\right\|=\left\|\left|\tau_{x} \cap \breve{A}_{1}\right|=\tilde{\omega}\right\|$ which implies $\left\|\left|\tau_{x} \cap \check{B}\right|=\tilde{\omega}\right\|=\left\|\left|\tau_{x} \cap A_{2}\right|=\tilde{\omega}\right\|$, witnessing that $A_{2}$ is a $x$ stable set.

Theorem 5.2.5 A c.B.a. $\mathbb{B}$ is limsup-stable iff it satisfies condition $(\hbar)$. 
Proof. $(\Rightarrow)$ Let $\mathbb{B}$ be a lim sup-stable algebra, i.e.

$$
\forall x \in \mathbb{B}^{\omega} \exists y \prec x \forall z \prec y \lim \sup z=\lim \sup y .
$$

Let $x \in \mathbb{B}^{\omega}$ and $S \in[\omega]^{\omega}$. Let $f: \omega \rightarrow S$ be the natural isomorphism. Then $x_{0}=x \circ f \in \mathbb{B}^{\omega}$, so, by (5.1), there exists $y \prec x_{0}$ such that

$$
\forall z \prec y \limsup z=\lim \sup y .
$$

Now $y=x_{0} \circ g$, for some $g \in \omega^{\dagger \omega}$ and clearly $A=f[g[\omega]] \in[S]^{\omega}$. Let $B \in[A]^{\omega}$. Since $C=g^{-1}\left[f^{-1}[B]\right] \in[\omega]^{\omega}$ there exists the natural isomorphism $h: \omega \rightarrow C$ and for $z=y \circ h=x \circ f \circ g \circ h$ we have $z \prec y$, so by (5.2) there holds $\lim \sup x \circ f \circ g \circ h=\limsup x \circ f \circ g$ that is, by Lemma 4.2.4(b), $\|\left[f[g[h[\omega]]]^{-} \cap\right.$ $\tau_{x} \mid=\tilde{\omega}\|=\|\left[f[g[\omega]]^{*} \cap \tau_{x} \mid=\tilde{\omega} \|\right.$. Since $B \subset f[g[\omega]]$ we have $f[g[h[\omega]]]=B$, hence $\left\|\left|\breve{B} \cap \tau_{x}\right|=\tilde{\omega}\right\|=\left\|\left|\breve{A} \cap \tau_{x}\right|=\tilde{\omega}\right\|$ and $(\hbar)$ holds.

$\left(\Leftrightarrow\right.$ Let $(\hbar)$ hold and $x \in \mathbb{B}^{\omega}$. Then there is $A \in[\omega]^{\omega}$ such that

$$
\forall B \in[A]^{\omega}\left\|\left|\bar{B} \cap \tau_{x}\right|=\dot{\omega}\right\|=\left\|\left|\check{A} \cap \tau_{x}\right|=\breve{\omega}\right\| .
$$

If $f: w \rightarrow A$ is the natural isomorphism, then $y=x \circ f \prec x$. For an arbitrary $z \prec y$ there exists $g \in \omega^{i \omega}$ such that $z=y \circ g$ and for $f[g[\omega]] \in[A]^{\omega}$, according to $(5.3)$, we have $\left\|\left|f[g[\omega]]^{-} \cap \tau_{x}\right|=\bar{\omega}\right\|=\left\|\left|f[\omega]^{\sim} \cap \tau_{x}\right|=\omega\right\|$ that is, by Lemma 4.2.4(b), $\lim \sup x \circ f \circ g=\lim \sup x \circ f$. Hence $\lim \sup z=\lim \sup y$.

Lemma 5.2.6 A c.B.a. $\mathbb{B}$ satisfies $(\hbar)$ if and only if for each sequence $x \in \mathbb{B}^{\omega}$ the set

$$
\Delta_{x}=\left\{A \in[\omega]^{\omega}: \forall B \in[A]^{\omega}\left\|\check{B} \subset^{*} \tau_{x}\right\|=\left\|\tilde{A} \subset^{*} \tau_{x}\right\|\right\}
$$

is dense in the partial order $\left\langle[\omega]^{\omega}, C\right\rangle$.

Proof. Firstly we prove that for each sequence $x \in \mathbb{B}^{\omega}$ and each $A, B \in[\omega]^{\omega}$ there holds

$$
\left\|\left|\bar{B} \cap \tau_{x}\right|=\tilde{\omega}\right\|=\left\|\left|\tilde{A} \cap \tau_{x}\right|=\tilde{\omega}\right\| \Leftrightarrow\left\|\tilde{B} C^{*} \tau_{x^{\prime}}\right\|=\left\|\bar{A} \subset^{*} \tau_{x^{\prime}}\right\|,
$$

where $x^{\prime}=\left\langle x_{n}^{\prime}: n \in \omega\right\rangle$. The left-hand side equality holds iff $\left\|\left|\check{B} \cap \tau_{x}\right|<\bar{\omega}\right\|=$ $\left\|\left|\tilde{A} \cap \tau_{x}\right|<\tilde{\omega}\right\|$, that is $\left\|\dot{B} \subset^{*} \breve{\omega} \backslash \tau_{x}\right\|=\left\|\tilde{A} C^{*} \breve{\omega} \backslash \tau_{x}\right\|$ which is, by Lemma 4.2 .4 , equivalent to the right-hand side equality and the $(5,4)$ is proved. Hence

$$
\forall x \in \mathbb{B}^{\omega} \quad D_{x}=\Delta_{x^{\prime}} .
$$

Let condition $(\hbar)$ hold, and let $x \in \mathbb{B}^{\omega}$. Then $x^{\prime} \in \mathbb{B}^{\omega}$, and because of $(\hbar), D_{x^{\prime}}$ is a dense subset of $[\omega]]^{\omega}$. By (5.5) the set $\Delta_{x}$ is dense in $[\omega]^{\omega}$ and " $\Rightarrow "$ is proved. The proof of the another implication is analogous. 


\subsection{Condition $(\hbar)$ and cellularity}

Theorem 5.3.1 [3] If a c.B.a. $\mathbb{B}$ satisfies the $t-c c$, then it satisfies $(\hbar)$.

Proof. Suppose that $\mathbb{B}$ does not satisfy $(\hbar)$. Then there exists $S \in[\omega]^{\omega}$ such that

$$
\forall A \in[S]^{\omega} \exists B \in[A]^{\omega}\left\|\left|\check{B} \cap \tau_{x}\right|=\omega\right\|<\left\|\left|\check{A} \cap \tau_{x}\right|=\dot{\omega}\right\| .
$$

Using the elementary properties of forcing we easily prove that for each $A, B \in$ $[\omega]^{\omega}$ we have

$$
\left(\left(B \subset^{*} A\right) \wedge\left(\left\|\left|\hat{B} \cap \tau_{x}\right|=\omega\right\|<\left\|\left|\tilde{A} \cap \tau_{x}\right|=\omega\right\|\right)\right) \Rightarrow\left(B \complement^{*} A\right) .
$$

Using recursion we define a sequence of sets $A_{\alpha} \in[S]^{\omega}, \alpha<\mathrm{t}$, such that

$$
\alpha<\beta \Rightarrow\left(A_{\beta} \check{\Sigma}^{*} A_{\alpha}\right) \wedge\left(\left\|\left|\tilde{A}_{\beta} \cap \tau_{x}\right|=\tilde{\omega}\right\|<\left\|\left|\tilde{A}_{\alpha} \cap \tau_{x}\right|=\tilde{\omega}\right\|\right) .
$$

Let $A_{0}=S$. Let $\xi<\mathrm{t}$ and let us define $A_{\alpha}, \alpha<\xi$, such that (5.8) holds.

If $\xi=\alpha+1$, then $A_{\alpha} \in[S]^{\omega}$, and, becouse of (5.6), we can choose $A_{\xi} \in\left[A_{\alpha}\right]^{\omega}$ such that $\left\|\left|\check{A}_{\xi} \cap \tau_{x}\right|=\bar{\omega}\right\|<\left\|\left|\tilde{A}_{\alpha} \cap \tau_{x}\right|=\dot{\omega}\right\|$. By (5.7), there holds $A_{\xi} \xi^{*} A_{\alpha}$. so the sequence $A_{\alpha}, \alpha \leq \xi$, satisfies (5.8).

If $\xi$ is a limit ordinal, then, since $\xi<\mathrm{t}$ and (by the hypothesis) $\left\langle\left\{A_{\alpha}: \alpha<\right.\right.$ $\left.\xi\}_{:}{ }^{*} \supset\right\rangle$ is a well-ordered set, the family $\left\{A_{\alpha}: \alpha<\xi\right\}$ has a pseudointersection $A \in[S]^{\omega}$, i.e.

$$
\forall \alpha<\xi A \subset^{*} A_{\alpha} \text {, }
$$

which implies

$$
\forall \alpha<\xi\left\|\left|\check{A} \cap \tau_{x}\right|=\omega\right\| \leq\left\|\left|\check{A}_{\alpha} \cap \tau_{x}\right|=\omega\right\| .
$$

Now, using (5.6), we choose $A_{\xi} \in[A]^{\omega}$ such that $\left\|\left|\check{A}_{\xi} \cap \tau_{x}\right|=\dot{\omega}\right\|<\|\left|\breve{A} \cap \tau_{x}\right|=$ $\breve{\omega} \|$. By (5.7) this implies $A_{\xi} \varsubsetneqq^{*} A$ which, according to (5.9) and (5.10), implies

$$
\forall \alpha<\xi\left(A_{\xi} \xi^{*} A_{\alpha} \wedge\left\|\left|\tilde{A}_{\xi} \cap \tau_{x}\right|=\breve{\omega}\right\|<\left\|\left|\tilde{A}_{\alpha} \cap \tau_{x}\right|=\check{\omega}\right\|\right) .
$$

Hence the sequence $A_{\alpha}, \alpha \leq \xi$, satisfies (5.8) again.

So, for $b_{\alpha}=\left\|\left|\vec{A}_{\alpha} \cap \tau_{x}\right|=\omega\right\|$ we obtain a decreasing sequence $\left\langle b_{\alpha}: \alpha<t\right\rangle$ in $\mathbb{B}$. Therefore the set $\left\{b_{\alpha} \backslash b_{\alpha+1} ; \alpha<t\right\} \subset \mathbb{B}^{+}$is an antichain in $\mathbb{B}$ witnessing $\mathbb{B}$ is not $t$-cc.

Theorem 5.3.2 If a c.B,a. $\mathbb{B}$ satisfies $(\hbar)$, then it satisfies the $\mathfrak{s}$-cc. 
Proof, Suppose $\mathbb{B}$ is not s-cc. Let $\mathcal{A}=\left\{b_{\alpha}: \alpha<s\right\} \subset \mathbb{B}^{+}$be a maximal antichain of size s and let $S=\left\{S_{\alpha}: \alpha<s\right\} \subset[\omega]^{\omega}$ be a splitting family. We define a name for a subset of $\omega, \tau$, by

$$
\tau=\left\{\left\langle\tilde{n}, \bigvee\left\{b_{\alpha}: \alpha<s \wedge n \in S_{\alpha}\right\}\right\rangle: n \in \omega\right\} .
$$

Firstly we prove that for each $\alpha<s$ there holds

$$
b_{\alpha} \Vdash \tau=\dot{S}_{\alpha} .
$$

Let $b_{\alpha_{0}} \in G$, where $G$ is a $\mathbb{B}$-generic filter over $V$. If $n \in \tau_{G}$, then $x_{n}=\bigvee\left\{b_{\alpha x}\right.$ : $\left.\alpha<5 \wedge n \in S_{\alpha}\right\} \in G$, which implies that $b_{\alpha_{0}} \wedge x_{n} \in G$ and consequently there is $\alpha<5$ such that $n \in S_{\alpha}$ and $b_{\alpha_{0}} \wedge b_{\alpha}>0$. This implies $\alpha=\alpha_{0}$ and $n \in S_{\alpha_{0}}$. Conversely, if $n \in S_{\alpha_{0}}$, then $b_{\alpha_{0}} \leq x_{n}$, thus $x_{n} \in G$ and $n \in \tau_{G}$. So $\tau_{G}=S_{\alpha_{0}}$ and $(5.11)$ is proved.

Let us prove

$$
\forall A \in[\omega]^{\omega} \exists B \in[A]^{\omega}\||\tilde{B} \cap \tau|=\omega\|<\||\tilde{A} \cap \tau|=\dot{\omega}\| .
$$

Let $A \in[\omega]^{\omega}$ and let $S_{\alpha_{0}}$ split $A$. Then $B=A \backslash S_{\alpha_{0}} \in[A]^{\omega}$, so

$$
\|\tilde{B} \cap \tau|=\check{\omega}\|\leq\|| \check{A} \cap \tau \mid=\check{\omega}\| .
$$

Also we have $\left|A \cap S_{\alpha_{0}}\right|=\omega$ and, by (5.11), $b_{\alpha_{0}} \Vdash \tau=\check{S}_{\alpha_{0}}$, thus $b_{\alpha_{0}} \Vdash \mid \check{A} \cap$ $\tau \mid=\check{\omega}$, that is $b_{\alpha_{0}} \leq\||\tilde{A} \cap \tau|=\tilde{\omega}\|$. Suppose the equality holds in (5.13). Then, by the last inequality, $b_{\alpha_{0}} \Vdash|\dot{B} \cap \tau|=\tilde{\omega}$ and, according to (5.11) we have $b_{\alpha_{0}} \Vdash\left|\tilde{B} \cap \breve{S}_{\alpha_{0}}\right|=\tilde{\omega}$, which is impossible. Thus we have " $<$ " in (5.13) and (5.13) is proved. Now, by (5.12), the sequence $x=\left\langle x_{n}: n \in \omega\right\rangle$ witnesses $\mathbb{B}$ does not satisfy $(\hbar)$.

Theorem 5.3.3 Let $\mathbb{B}$ be an $(w, 2)$-distributive c,B,a. Then $\mathbb{B}$ satisfies $(\hbar)$ if and only if $\mathbb{B}$ is $\mathbf{s - c c}$.

Proof. By the previous theorem the implication " $\Leftarrow$ " remains to be proved. Suppose $\neg(\hbar)$. Then there exist $x \in \mathbb{B}^{\omega}$ and $S \in[\omega]^{\omega}$ such that

$$
\forall A \in[S]^{\omega} \exists B \in[A]^{\omega}||\left|\bar{A} \cap \tau_{x}\right|=\check{\omega} \wedge\left|\hat{B} \cap \tau_{x}\right|<\omega \|>0 .
$$

Since $\mathbb{B}$ is $(\omega, 2)$-distributive there holds $1 \Vdash \tau_{I} \in\left(P(\omega)^{V}\right)$. Let

$$
\mathcal{T}=\left\{C \in[\omega]^{\omega}:\left\|\tau_{x}=\hat{C}\right\|>0\right\}
$$


and for $C \in \mathcal{T}$ let $b_{C}=\left\|\tau_{x}=\tilde{C}\right\|$. Firstly we prove that $\left\{b_{C}: C \in \mathcal{T}\right\}$ is a maximal antichain below $b=\left\|\left|\tau_{x}\right|=\omega\right\|$. Clearly $b_{C} \leq b$, for all $C \in \mathcal{T}$ and $b_{C_{1}} \wedge b_{C_{2}}=0$, for different $C_{1}, C_{2} \in \mathcal{T}$. Since $1 \mathbb{I f} \tau_{x} \in\left(P(\omega)^{V}\right)$ we have $b \Vdash \exists C \in\left(\left([\omega]^{\omega}\right)^{V}\right) \tau_{x}=C$, that is $\forall p \in(0, b] \exists C \in[\omega]^{\omega} p \wedge b_{C}>0$ and the maximality is proved.

Now we prove $\mathcal{T}$ is a splitting family on the set $S$. Let $A \in[S]^{\omega}$. By (5.14) there is $B \in[A]^{\omega}$ such that $b^{*}=\left\|\left|\tilde{A} \cap \tau_{x}\right|=\check{\omega} \wedge\left|\tilde{B} \cap \tau_{x}\right|<\tilde{\omega}\right\|>0$. Clearly, $b^{*} \leq b$, so there is $C_{0} \in \mathcal{T}$ such that $b^{*} \wedge b_{C_{0}}>0$. Let $G$ be a $\mathbb{B}$-generic filter containing $b^{*} \wedge b_{C_{0}}$. Then, in $V_{\mathbb{B}}[G]$, we have $\left(\tau_{x}\right)_{G}=C_{0},\left|\left(\tau_{x}\right)_{G} \cap A\right|=\omega$ and $\left|\left(\tau_{x}\right)_{G} \cap B\right|<\omega$, which implies that $C_{0}$ splits $A$.

Since $|\mathcal{T}| \geq \mathbf{s},\left\{b_{C}: C \in \mathcal{T}\right\}$ is an antichain in $\mathbb{B}$ of size $\geq \mathbf{s}$, thus $\mathbb{B}$ is not a s-cc algebra.

Corollary 5.3.4 (a) Let $\kappa \geq \omega$ be a cardinal. The algebra $P(\kappa)$ is not $\kappa$-cc and satisfies $(\hbar)$ if and only if $\kappa<\mathbf{s}$.

(b) The implication " $(h) \Rightarrow \mathrm{s}-\mathrm{cc}$ " is the best possible in ZFC, more precisely

$$
\mathfrak{s}=\min \{\kappa \in \text { Card : Each c.B.a. having }(\hbar) \text { has the } \kappa-\mathrm{cc}\} \text {. }
$$

(c) $\mathrm{ZFC} \forall \forall(\hbar) \Rightarrow \kappa-\mathrm{cc}$, for $\kappa \in\{\mathrm{t}, \mathfrak{h}, \mathfrak{b}\}$.

(d) ZFC $\not \forall$ b-cc $\Rightarrow(\hbar)$.

Proof. (a) Clearly $P(\kappa)$ is not a $\kappa$-cc algebra and it is $(\omega, 2)$-distributive. So, by Theorem 5.3.3, $P(\kappa)$ satisfies $(\hbar)$ iff $P(\kappa)$ is $\mathbf{5}$-cc iff $\kappa<\mathbf{s}$.

(b) The inequality " $\geq$ " follows from Theorem 5.3.2. For a proof of " $\leq$ " suppose $\kappa<\mathbf{s}$. Then, by (a), the algebra $P(\kappa)$ satisfies $(\hbar)$, but it is not $\kappa$-cc.

(c) Since ZFC $\vdash \mathrm{t} \leq \mathfrak{h} \leq \mathrm{b}$ (see [4]) and $\operatorname{Con}(\mathrm{b}<\mathrm{s}$ ) (see [12]), for $\kappa \in$ $\{t, \mathfrak{b}, \mathfrak{b}\}$ we have $\operatorname{Con}(\kappa<s)$. So in a model of $\kappa<s$ the algebra $P(\kappa)$ has $(\hbar)$ (by (a)) but it is not $\kappa$-cc.

(d) It is well-known that $\operatorname{Con}(\mathbf{s}<\mathbf{b})$ (see, for example, [45]). So in a model of $s<b$ the algebra $P(s)$ is $b$-cc but it does not satisfy condition $(\hbar)$ by (a).

Corollary 5.3.5 Conditions ( $\hbar$ ) and cec are equivalent (in the class of all c.B.a's) if and only if $s=\omega_{1}$.

Proof. Let $(\hbar) \Leftrightarrow \mathrm{ccc}$ and suppose $\mathrm{s}>\omega_{1}$. Then, by Theorem 5.3.3, $P\left(\omega_{1}\right)$ satisfies $(\hbar)$, that is ccc, which is not true. Thus $s=\omega_{1}$. Conversely, if $s=\omega_{1}$, then clearly $t=\omega_{1}$ and using Theorems 5.3.1 and 5.3.2 we obtain $(\hbar) \Leftrightarrow \omega_{1}$-cc.

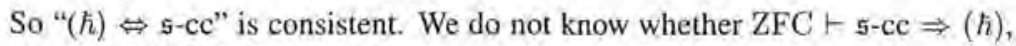
even whether $\mathrm{ZFC} \vdash \mathrm{h}-\mathrm{cc} \Rightarrow(\hbar)$. 


\subsection{A posteriori convergence}

In spaces of the form $\left\langle\mathbb{B}_{,} \tau_{s}\right\rangle$ the a priori and the a posteriori convergence must not coincide. We have already mentioned that a sequence converges a posteriori iff each subsequence has a subsequence which converges a priori. In the next theorem several equivalent conditions for the a posteriori convergence in $\tau_{5}$ will be given.

Theorem 5.4.1 Let $\mathbb{B}$ a c.B.a. and $x \in \mathbb{B}^{\omega}$. Then the following conditions are equivalent:

(a) $\lim x=b$;

(b) $\forall y \prec x \exists z \prec y z \rightarrow b$;

(c) $\forall f \in \omega^{\dagger \omega} \exists g \in \omega^{\dagger \omega} \lim \sup x \circ f \circ g=\liminf x \circ f \circ g=b$;

(d) $\forall A \in[\omega]^{\omega} \exists B \in[A]^{\omega}\left\|\left|\tau_{x} \cap \check{B}\right|=\check{\omega}\right\|=\left\|\tilde{B} C^{*} \tau_{x}\right\|=b$;

(e) $\forall A \in[\omega]^{\omega} \exists B \in[A]^{\omega} \lim \sup _{n \in B} x_{n}=\liminf _{n \in B} x_{n}=b$.

Proof. The equivalence (a) $\Leftrightarrow(\mathrm{b})$ is Theorem 5.1.1. Clearly, (c) is (b) and (d) is (e) written in another way.

(c) $\Rightarrow$ (d). Let condition (c) hold. Let $A \in[\omega]^{\omega}$ and let $f_{A}: \omega \rightarrow \omega$ be the increasing enumeration of the set $A$. Then, by (c), there is a function $g \in \omega^{\text {tw }}$ such that

$$
\limsup x \circ f_{A} \circ g=\liminf x \circ f_{A} \circ g=b .
$$

Since $f_{A}$ and $g$ are injections we have $B \stackrel{\text { def }}{=} f_{A}[g[\omega]] \in[A]^{\omega}$ and using Theorem 4.2.2 and (5.15) we obtain $\left\|\left|\breve{B} \cap \tau_{x}\right|=\breve{\omega}\right\|=\left\|\breve{B} \subset^{*} \tau_{x}\right\|=b$.

(d) $\Rightarrow$ (c). Let (d) hold, let $f \in \omega^{\top \omega}$ and $A=f[\omega]$. Then by (d) there exists $B \in[A]^{\omega}$ such that

$$
\left\|\left|\tilde{B} \cap \tau_{x}\right|=\check{\omega}\right\|=\left\|\check{B} \subset^{*} \tau_{x}\right\|=b .
$$

Let $C=f^{-1}[B]$ and let $g_{C}: \omega \rightarrow \omega$ be the increasing enumeration of the set $C$. Since $B \subset f[\omega]$ we have $f\left[g_{C}[\omega]\right]=B$, so, by Theorem 4.2.2 and (5.16) there holds limsup $x \circ f \circ g_{C}=\lim \inf x \circ f \circ g_{C}=b$ and (c) is proved.

Definition 5.4.2 For a sequence $x \in \mathbb{B}^{\omega}$ and element $b \in \mathbb{B}$ let

$$
\begin{gathered}
D_{b}^{x}=\left\{B \in[\omega]^{\omega}:\left\|\left|\tau_{x} \cap \breve{B}\right|=\check{\omega}\right\|=\left\|\breve{B} \subset^{*} \tau_{x}\right\|=b\right\} \\
J_{b}^{x}=[\omega]^{<\omega} \cup D_{b}^{x}
\end{gathered}
$$

Lemma 5.4.3 The set $D_{b}^{x}$ is closed toward subsets and join in $[\omega]^{\omega}$. The set $J_{b}^{x}$ is equal to $P(\omega)$ or it is an ideal in $P(\omega)$. 
Proof. It is sufficient to prove that $J_{b}^{x}$ is $P(\omega)$ or an ideal, since $D_{b}^{x}=[\omega]^{\omega} \cap J_{b}^{x}$. Assuming $\mathcal{J}_{b}^{x} \neq P(\omega)$ we prove that $\mathcal{J}_{b}^{x}$ is an ideal.

(12) Let $C \subset B \in \mathcal{J}_{b}^{x}$. If $C$ is a finite set, then clearly $C \in \mathcal{J}_{b}^{x}$. Otherwise, since $1 \Vdash \tilde{B} \subset^{*} \tau_{x} \Rightarrow \mathscr{C} C^{*} \tau_{x} \Rightarrow\left|\check{C} \cap \tau_{x}\right|=\breve{\omega} \Rightarrow\left|\tilde{B} \cap \tau_{x}\right|=\tilde{\omega}$, by Fact 4.1.2(b), we have $b=\left\|\check{B} C^{*} \tau_{x}\right\| \leq\left\|\check{C} \subset^{*} \tau_{x}\right\| \leq\left\|\left|\check{C} \cap \tau_{x}\right|=\check{\omega}\right\| \leq\left\|\left|\tilde{B} \cap \tau_{x}\right|=\tilde{\omega}\right\|=b$, thus $C \in \mathcal{J}_{b}^{x}$.

(I1) Clearly, $\emptyset \in \mathcal{J}_{b}^{x}$ and, by (I2), $\omega \in \mathcal{J}_{b}^{x}$ would imply $\mathcal{J}_{b}^{x}=P(\omega)$, thus $\omega \notin \mathcal{J}_{b}^{x}$.

(I3) Assuming $B, C \in \mathcal{J}_{b}^{x}$ we will prove that $B \cup C \in \mathcal{J}_{b}^{x}$. If the sets $B$ and $C$ are infinite, then $\left\|\left|(B \cup C) \cap \tau_{x}=\tilde{\omega}\|=\|\right| \tilde{B} \cap \tau_{x}|=\dot{\omega}\|\vee\|| C \cap \cap \tau_{x} \mid=\tilde{\omega}\right\|=b \vee b=$ $b$ and $\left\|(B \cup C)^{*} C^{*} \tau_{x}\right\|=\left\|\tilde{B} C^{*} \tau_{x}\right\| \wedge\left\|\tilde{C} \subset^{*} \tau_{x}\right\|=b \wedge b=b$, so $B \cup C \in \mathcal{J}_{b}^{x}$. If the sets $B$ and $C$ are finite, then $B \cup C \in[\omega]^{<\omega} \subset \mathcal{J}_{b}^{x}$. Finally, if exactly one of two sets, say $B$, is finite, then $\left\|\left|(B \cup C) \tau \tau_{x}\right|=\tilde{\omega}\right\|=\left\|\left|\tilde{C} \cap \tau_{x}\right|=\tilde{\omega}\right\|=b$ and $\left\|(B \cup C)^{*} \mathcal{C}^{*} \tau_{x}\right\|=\left\|C C^{*} \tau_{x}\right\|=b$, thus $B \cup C \in \mathcal{J}_{b}^{x}$ again.

Theorem 5.4.4 Let $\mathbb{B}$ be a c.B.a. For a sequence $x \in \mathbb{B}^{\omega}$ and element $b \in \mathbb{B}$ we have:

(i) the following conditions are equivalent:

(a) $x \rightarrow_{\tau_{s}} b$ (converges a posteriori);

(b) $D_{b}^{x}$ is dense in $\left.\langle[\omega]]^{\omega}, C\right\rangle$;

(c) $J_{b}^{x} \in\{P(\omega)\} \cup \operatorname{Tall}(\omega)$.

(ii) the following conditions are equivalent:

(a) $x \rightarrow b$ (converges a priori);

(b) $D_{b}^{x}=[\omega]^{\omega}$;

(c) $J_{b}^{x}=P(\omega)$.

(iii) the following conditions are equivalent:

(a) $x \rightarrow \tau_{s} b$ and $x+b$;

(b) $J_{b}^{*} \in \operatorname{Tall}(\omega)$.

(iv) If $x \rightarrow_{\tau_{A}} b$ and $x \nrightarrow b$ then there exists an extension $V_{\mathbb{B}}[G]$ in which real $\left(\tau_{x}\right)_{G}$ or $\omega \backslash\left(\tau_{x}\right)_{G}$ kills $J_{b}^{x}$,

Proof. (ii) Since, $[\omega]^{\omega} \cup[\omega]^{<\omega}=P(\omega)$, (b) is equivalent to (c). Let us prove that (a) is equivalent to (c). According to the definition of the algebraic convergence, $x \rightarrow b$ if and only if $\lim \sup x=\lim \inf x=b$, that is, by Theorem $4.2,2, \|\left|\tau_{x}\right|=$ $\check{\omega}\|=\| \check{\omega} \subset^{*} \tau_{x} \|=b$, which is equivalent to $\omega \in \mathcal{J}_{b}^{x}$, and, by Lemma 5.4.3, to $\mathcal{J}_{b}^{x}=P(\omega)$.

(i) Equivalence between (b) and (c) follows directly from Fact 3.2.2 (b). Now, let us prove that (a) $\Leftrightarrow$ (c). Let $x \rightarrow \tau_{s} b$. If $\mathcal{J}_{b}^{x}=P(\omega)$ we are done. Otherwise, by Lemma 5.4.3, $\mathcal{J}_{b}^{x}$ is an ideal in $P(\omega)$ and by (d) of Theorem 5.4.1 it is tall. 
Conversely, if $\mathcal{J}_{b}^{x}$ is a tall ideal, then (d) of Theorem 5.4.1 holds and $x \rightarrow \tau_{\tau_{s}} b$. Otherwise $\mathcal{J}_{b}^{x}=P(\omega)$ so, by $($ ii $), x \rightarrow b$, which implies that $x \rightarrow \tau_{a} b$.

(iii) Follows directly from (i) and (ii).

(iv) Let $x \rightarrow \tau_{s} b$ and $x \not b$. For each $B \in \mathcal{J}_{b}^{x} \cap[\omega]^{\omega}$ we have lim inf $x=$ $\left\|\tilde{\omega} \subset^{*} \tau_{x}\right\| \leq\left\|\tilde{B} \subset^{*} \tau_{x}\right\|=b=\left\|\left|\tilde{B} \cap \tau_{x}\right|=\tilde{\omega}\right\| \leq\left\|\left|\tau_{x}\right|=\tilde{\omega}\right\|=\limsup x$. Since $x+b$ we have lim inf $x<\limsup x$, so liminf $x<b$ or $b<\lim \sup x$.

If $\liminf x<b$, then $c=b \wedge\left\||\bar{\omega}| \tau_{\infty} \mid=\bar{\omega}\right\|>0$. Now $c \leq b$ implies $c \leq\left\|\bar{B} \subset^{*} \tau_{x}\right\|$, for each $B \in \mathcal{J}_{b}^{x} \cap[\omega]^{\omega}$ which means that $c \Vdash \forall B \in \mathcal{J}_{b}^{x}|B \cap \omega|$ $\tau_{x} \mid<\bar{\omega}$. Thus if $G$ is $\mathbb{B}$-generic filter and $c \in G$, then in $V_{\mathbb{B}}[G]$ the set $\omega \backslash\left(\tau_{x}\right)_{G}$ is infinite and kills the tall ideal $\mathcal{J}_{b}^{x}$.

If $b<\limsup x$, then $c=b^{\prime} \wedge\left\|\left|\tau_{x}\right|=\tilde{\omega}\right\|>0$. Now $c \leq b^{\prime}$ implies $c \leq\left\|\left|\bar{B} \cap \tau_{x}\right|<\check{\omega}\right\|$, for all $B \in \mathcal{J}_{b}^{x} \cap[\omega]^{\omega}$ which implies that $c \Vdash \forall B \in$ $\mathcal{J}_{b}^{x}\left|B \cap \tau_{x}\right|<\tilde{\omega}$. So $c$ forces that the set $\tau_{x}$ is infinite and kills $\mathcal{J}_{b}^{x}$.

Lemma 5.4.5 If for elements $b, c \in \mathbb{B}$ the sets $D_{b}^{x}$ and $D_{c}^{x}$ are dense in $[\omega]^{\omega}$, then $b=c$.

Proof. Let us suppose that $b \neq c$ and let $B \in D_{b}^{x}$. Since $D_{c}^{x}$ is dense in $[\omega]^{\omega}$, there exists $C \in D_{c}^{x}$ such that $C \subset B$. Since, from Lemma $5,4,3, D_{b}^{x}$ is closed toward subsets, we have $C \in D_{b}^{x}$. Hence, $C \in D_{b}^{x} \cap D_{c}^{x}$, which implies that $b=\left\|\tilde{C} \subset^{*} \tau_{x}\right\|=c$. A contradiction.

Remark 5.4.6 Lemma 5.4.5 implies that the topological limit is unique. Let us remind that this holds in Hausdorff spaces, but the converse is not true.

Lemma 5.4.7 Let $\mathbb{B}$ be a c.B.a., $y \in \mathbb{B}^{\omega}$ and $\mathcal{J} \subset P(\omega)$ a tall ideal. If $c=\|\left|\tau_{y}\right|=$ $\tilde{\omega} \wedge \forall B \in \tilde{\mathcal{J}}\left|B \cap \tau_{y}\right|<\tilde{\omega} \|>0$, then the sequence $x=\left\langle y_{n} \wedge c: n \in \omega\right\rangle$ converges to 0 a posteriori, but not a priori.

Proof. Clearly $c^{\prime} \Vdash \tau_{x}=\bar{\emptyset}$ and $c \| \tau_{x}=\tau_{y}$, because whenever $G$ is a $\mathbb{B}$-generic filter containing $c$ there holds: $n \in\left(\tau_{x}\right)_{G}$ iff $y_{n} \wedge c \in G$ iff $y_{n} \in G$ iff $n \in\left(\tau_{y}\right)_{G}$. Consequently, in each generic extension $\left.V_{B} \mid G\right]$ the set $\left(\tau_{x}\right)_{G}$ is either empty or almost disjoint with each $B \in \mathcal{J}$, thus $\left(\tau_{x}\right)_{G}$ is not a cofinite set and, by Theorem 4.2.2, lim inf $x=0$. On the other hand, limsup $x=c \wedge \lim \sup y=c \wedge||\left|\tau_{y}\right|=$ $\tilde{\omega} \|=c>0$, thus the sequence $x$ does not converge a priori.

Let us prove $x \rightarrow \tau_{0} 0$, that is (by Theorem 5.4.1)

$$
\forall A \in[\omega]^{\omega} \exists B \in[A]^{\omega}\left\|\left|\vec{B} \cap \tau_{x}\right|=\check{\omega}\right\|=\left\|\breve{B} \subset^{*} \tau_{x}\right\|=0 .
$$

Let $A \in[\omega]^{\omega}$. Since $\mathcal{J}$ is a tall ideal, there exists $B \in \mathcal{J} \cap[A]^{\omega}$. Now $c \Vdash \tau_{x}=\tau_{y}$ implies $c \leq\left\|\left|\tilde{B} \cap \tau_{x}\right|<\dot{\omega}\right\|$ and $c^{\prime} \Vdash \tau_{x}=\dot{\emptyset}$ implies $c^{\prime} \leq\left\|\left|\dot{B} \cap \tau_{x}\right|<\dot{\omega}\right\|$. 
Hence $\left\|\left|\check{B} \cap \tau_{x}\right|<\bar{\omega}\right\|=1$, that is $\left\|\left|\check{B} \cap \tau_{x}\right|=\tilde{\omega}\right\|=0$. Since $|B|=\omega$, we have $\left\|\dot{B} \subset^{*} \tau_{x}\right\| \leq\left\|\tilde{B} \cap \tau_{x} \mid=\tilde{\omega}\right\|$ which implies that $\left\|\check{B} \subset^{*} \tau_{x}\right\|=0$ and (5.17) is proved.

Theorem 5.4.8 A complete Boolean algebra is $(\omega, 2)$-distributive iff the a priori limit operator coincides with the a posteriori limit operator.

The direction $(\Rightarrow)$ follows from Theorem 5.4 .4 (iv).

$(\nLeftarrow)$ Suppose $\mathbb{B}$ is not $(\omega, 2)$-distributive. Then in some generic extension, $V_{\mathbb{B}}[G]$, a new real appears and, by Lemma 4.2 .1 , there is a real $r \in V_{\mathbb{B}}[G]$ which kills some tall ideal $\mathcal{J} \subset P(\omega)$. Let $y_{n}=\|\tilde{n} \in r\|, n \in \omega$. Then the assumptions of Lemma 5.4.7 are satisfied and two convergences are not equal.

Theorem 5.4.9 Let $\mathbb{B}$ be a c.B.a., $x \in \mathbb{B}^{\omega}$ be a sequence in $\mathbb{B}$ and $a_{x}$ and $b_{x}$ as in Definition 4.2.5. Then

$$
x \rightarrow \tau_{s} b \Rightarrow a_{x}=b_{x}=b
$$

Proof. Let $x \rightarrow \tau_{s} b$, that is, by Theorem 5.4.1,

$$
\forall A \in[\omega]^{\omega} \exists B \in[A]^{\omega}\left\|\left|\tilde{B} \cap \tau_{x}\right|=\tilde{\omega}\right\|=\left\|\dot{B} c^{*} \tau_{x}\right\|=b .
$$

So, for an arbitrary $A \in[\omega]^{\omega}$ there is $B_{A} \in[A]^{\omega}$ such that $\left\|\left|\breve{B}_{A} \cap \tau_{x}\right|=\bar{\omega}\right\|=b$ which implies that $\bigwedge_{B \in[A] \omega}\left\|\left|\tilde{B} \cap \tau_{x}\right|=\bar{\omega}\right\| \leq b$. So, by Lemma $4.2 .6, b_{x} \leq b$. By $(5,19)$ again, for an arbitrary $A \in[\omega]^{\omega}$ there is $B_{A} \in[A]^{\omega}$ such that $\| \breve{B}_{A} C^{*}$ $\tau_{x} \|=b$, so $\bigvee_{B \in \mid A]^{*}}\left\|\dot{B} C^{*} \tau_{x}\right\| \geq b$, thus by Lemma 4.2 .6 we have $a_{x} \geq b$, so $a_{x} \geq b \geq b_{x}$. Finally, according to Lemma 4.2 .7 there holds $a_{x}=b_{x}=b$.

The following theorem shows that the converse is not true.

Theorem 5.4.10 Let $\mathbb{B}$ be a c.B.a. which has an antichain of size $c$ and produces new reals in each generic extension. Then there is a sequence $x$ in $\mathbb{B}$ which does not converge a posteriori although $a_{x}=b_{x}$.

Proof. By Lemma 4.2.1 there is a madf $\mathcal{A}$ on $w$ which is killed in each generic extension containing new reals. Thus, by the assumption, $1 \Vdash \exists r\left(r \in[\bar{\omega}]^{\omega} \wedge \forall A \in\right.$ $\check{A}|r \cap A|<\bar{\omega}$ ) and, by The Maximum Principle (see Fact $4.1 .2(\mathrm{e})$ ), there is a $\mathbb{B}$ name, $\pi$, such that

$$
1 \Vdash \pi \in[\ddot{\omega}]^{\omega} \wedge \forall A \in \dot{A}|\pi \cap A|<\tilde{\omega} .
$$

Let us fix an enumeration $[\omega]^{\omega}=\left\{S_{\alpha}: \alpha<c\right\}$ and choose bijections $f_{\alpha}: S_{\alpha} \rightarrow$ $\omega, \alpha<c$. Let $\left\{b_{\alpha}: \alpha<c\right\} \subset \mathbb{B}^{+}$be a maximal antichain in $\mathbb{B}$ and let the name $\sigma$ be defined by $\sigma=\left\{\left\langle\hat{n}, \bigvee_{\alpha<\epsilon} b_{\alpha} \wedge\left\|f_{\alpha}(n) \in \pi\right\|\right\rangle: n \in \omega\right\}$. 
Claim 1. $b_{\alpha} \Vdash \sigma=f_{\alpha}^{-1}[\pi]$.

Proof of Claim 1. Let $G$ be a $\mathbb{B}$-generic filter over $V$ and $b_{\alpha_{0}} \in G$. Then $n \in \sigma_{G}$ iff $b_{\alpha_{0}} \wedge \bigvee_{\alpha<c} b_{\alpha} \wedge\left\|f_{\alpha}(n) \in \pi\right\| \in G$ iff $b_{\alpha_{0}} \wedge\left\|f_{\alpha_{0}}(n) \in \pi\right\| \in G$ iff $\| f_{\alpha_{0}}(n) \in$ $\pi \| \in G$ iff $f_{\alpha_{0}}(n) \in \pi_{G}$ iff $n \in f_{\alpha_{0}}^{-1}\left[\pi_{G}\right]$. Thus $\sigma_{G}=f_{\alpha_{0}}^{-1}\left[\pi_{G}\right]$ and Claim 1 is proved.

Clearly, for $\alpha<c, \mathcal{A}_{\alpha}=\left\{f_{\alpha}^{-1}[A]: A \in \mathcal{A}\right\}$ is a mad family on the set $S_{\alpha}$.

Claim 2. $b_{\alpha} \Vdash \sigma \in\left[\tilde{S}_{\alpha}\right]^{\breve{\omega}} \wedge \forall A \in \tilde{\mathcal{A}}_{\alpha}|\sigma \cap A|<\check{\omega}$.

Proof of Claim 2. According to (5.20) and Claim 1 we have $b_{\alpha} \Vdash \sigma \in\left[\tilde{S}_{\alpha}\right]^{\omega}$. Let $b_{\alpha} \in G$ and $A \in \mathcal{A}_{\alpha}$. Then $A=f_{\alpha}^{-1}\left[A_{1}\right]$ for some $A_{1} \in \mathcal{A}$ and, by (5.20) we have $\left|\pi_{G} \cap A_{1}\right|<\omega$ which, since $f_{\alpha}$ is a bijection, implies $\left|f_{\alpha}^{-1}\left[\pi_{G}\right] \cap A\right|<\omega$, that is, by Claim $1,\left|\sigma_{G} \cap A\right|<\tilde{\omega}$ and Claim 2 is proved.

Claim 3. $1 \Vdash \forall A \in\left(\left([\omega]^{\omega}\right)^{V}\right) \exists B \in\left(\left([A]^{\omega}\right)^{V}\right)|\sigma \cap B|<\omega$.

Proof of Claim 3. Let $G$ be a $\mathbb{B}$-generic filter over $V$. Then there is exactly one $\alpha_{0}<\mathrm{c}$ such that $b_{\alpha_{0}} \in G$ and, by Claim 2,

$$
\sigma_{G} \in\left[S_{\alpha_{0}}\right]^{\omega} \wedge \forall A \in \mathcal{A}_{\alpha_{0}}\left|\sigma_{G} \cap A\right|<\omega .
$$

Let $A \in\left([\omega]^{\omega}\right)^{V}$. Then there is $\alpha_{1}<\mathrm{c}$ such that $A=S_{\alpha_{1}}$. If $\left|S_{\alpha_{1}} \cap S_{\alpha_{0}}\right|<\omega$, then, by (5.21), we have $\left|S_{\alpha_{1}} \cap \sigma_{G}\right|<\omega$ that is for $B=A$ there holds $\left|\sigma_{G} \cap B\right|<\omega$. Otherwise, if $\left|S_{\alpha_{1}} \cap S_{\alpha_{0}}\right|=\omega$, then, since $\mathcal{A}_{\alpha_{0}}$ is a mad family on the set $S_{\alpha_{0}}$, there is $C \in \mathcal{A}_{\alpha_{0}}$ such that the set $B=C \cap S_{\alpha_{1}} \cap S_{\alpha_{0}}$ is infinite. By (5.21) $\left|\sigma_{G} \cap C\right|<\omega$ which implies that $\left|\sigma_{G} \cap B\right|<\omega$ and Claim 3 is proved.

Clain 4. $\forall A \in[\omega]^{\omega}\||\check{A} \cap \sigma|=\breve{\omega}\|>0$.

Proof of Claim 4. If $A \in[\omega]^{\omega}$ then $A=S_{\alpha_{0}}$ for some $\alpha_{0}<\mathrm{c}$. By Claim 2, $b_{\alpha_{0}} \Vdash \sigma \in[\tilde{A}]^{\tilde{\omega}}$ and consequently $b_{\alpha_{0}} \Vdash|\breve{A} \cap \sigma|=\tilde{\omega}$. By the assumption $b_{\alpha_{0}}>0$ and Claim 4 is proved.

Let $x_{n}=\|\tilde{n} \in \sigma\|^{\prime}, n \in \omega$. Then, by Theorem 4.2.2, $1 \Vdash \tau_{x}=\bar{\omega} \backslash \sigma$, hence, by Claim 3 we have $a_{x}=\left\|\forall A \in\left(\left([\omega]^{\omega}\right)^{V}\right) \exists B \in\left(\left([A]^{\omega}\right)^{V}\right) B C^{*} \tau_{x}\right\|=1$ and, according to Lemma $4.2 .7, b_{x}=1$.

Suppose $x \rightarrow_{\tau_{y}} b$. Then, by Theorem 5.4.9, $b=1$, so, by Theorem 5.4.1 there exists $A \in[\omega]^{\omega}$ such that $\left\|\tilde{A} \subset^{*} \tau_{x}\right\|=1$. But this is impossible because, by Claim $4,\left\|\left|\breve{A} \cap \breve{\omega} \backslash \tau_{x}\right|=\breve{\omega}\right\|>0$, thus the sequence $x$ does not converge a posteriori.

We can also characterize both convergences, the a priori and the a posteriori, using the notions of forcing.

Theorem 5.4.11 Let $\mathbb{B}$ be a c.B.a. and $x \in \mathbb{B}^{\omega}$. Then 
(a) $x$ converges a priori if and only if

$$
1 \text { it } \tau_{x} \text { is finite or cofinite. }
$$

(b) $x$ converges a posteriori if and only if there exists a tall ideal $\mathcal{J}$ on $\omega$ such that

$$
1 \Vdash \tau_{x} \text { is finite or cofinite or kills } \breve{\mathcal{J}} \text { or } \tau_{x}^{c} \text { kills } \breve{\mathcal{J}} \text {. }
$$

(c) If $x$ converges a posteriori, then $x \rightarrow \tau_{i} b$ where $b=\left\|\forall B \in \check{\mathcal{J}} B \subset^{*} \tau_{x}\right\|$ and there holds $\mathcal{J} \subset \mathcal{J}_{b}^{x}$.

Proof. (a) By definition, the sequence $x$ converges a priori iff lim inf $x=\lim \sup x$ which is, by Theorem 4.2 .2 equivalent to $\left\|\left|\tau_{x}\right|=\bar{\omega}\right\| \leq\left\||\bar{\omega}| \tau_{x} \mid<\bar{\omega}\right\|$ that is $1 \Vdash\left|\tau_{x}\right|<\tilde{\omega} \vee|\bar{\omega}| \tau_{x} \mid<\tilde{\omega}$.

(b) $\left(\Rightarrow\right.$ ) Let $x \rightarrow_{\tau_{s}} b$. If $x \rightarrow b$, then (5.22) holds, hence (5.23) holds trivially, for each tall ideal $\mathcal{J}$. Otherwise, if $x$ does not converge a priori, then, by Theorem 5.4.1 (iii), $\mathcal{J}_{b}^{x}$ is a tall ideal. By Theorem 5.4.9 we have $b=a_{x}=b_{x}$, which, by Lemma 4.2 .7 implies $\lim \inf x \leq b \leq \lim \sup x$. Thus $1=\lim \inf x \vee(b)$ $\lim \inf x) \vee(\lim \sup x \backslash b) \vee(1 \backslash \lim \sup x)$ is a partition of the unity (some members of which can be zero). In order to prove (5.23) suppose $G$ is a $\mathbb{B}$-generic filter over $V$. If $\liminf x \in G$ or $1 \backslash \limsup x \in G$, then $\left(\tau_{x}\right)_{G}$ is a cofinite or a finite set and we are done. If $b \backslash \liminf x \in G$, then $\omega \backslash\left(\tau_{x}\right)_{G}$ is an infinite set and for each $B \in \mathcal{J}_{b}^{x} \cap[\omega]^{\omega}$ we have $b=\left\|\tilde{B} \subset^{*} \tau_{x}\right\| \in G$, which implies that $\left|B \cap \omega \backslash\left(\tau_{x}\right)_{G}\right|<\omega$, for every $B \in \mathcal{J}_{b}^{x}$. Thus $\left(\tau_{x}\right)_{G}^{c}$ kills $\mathcal{J}_{b}^{x}$. Finally if $\limsup x \backslash b \in G$, then $\left(\tau_{x}\right)_{G}$ is an infinite set and for each $B \in \mathcal{J}_{b}^{x} \cap[\omega]^{\omega}$ there holds $b^{\prime}=\left\|\left|\tilde{B} \cap \tau_{x}\right|<\tilde{\omega}\right\| \in G$. So $\left|B \cap\left(\tau_{x}\right)_{G}\right|<\omega$ for all $B \in \mathcal{J}_{b}^{x}$, that is, $\left(\tau_{x}\right)_{G}$ kills the tall ideal $\mathcal{J}_{b}^{x}$ and $(5.23)$ is proved.

$(\Leftarrow)$ Let $\mathcal{J}$ be a tall ideal on $w$ such that $(5.23)$ holds. Let us prove the equality

$$
\left\|\forall B \in \check{\mathcal{J}} B \subset^{*} \tau_{x}\right\|=\left\|\exists B \in \check{\mathcal{J}}\left|B \cap \tau_{x}\right|=\check{\omega}\right\| .
$$

Let $B_{0} \in \mathcal{J} \cap[\omega]^{\omega}$. Then $\bigwedge_{B \in \mathcal{J}}\left\|\tilde{B} C^{*} \tau_{x}\right\| \leq\left\|\tilde{B}_{0} \subset^{*} \tau_{x}\right\| \leq\left\|\left|\tilde{B}_{0} \cap \tau_{x}\right|=\dot{\omega}\right\| \leq$ $\bigvee_{B \in \mathcal{J}}\left\|\left|\tilde{B} \cap \tau_{x}\right|=\tilde{\omega}\right\|$ so we proved " $\leq$ " in (5.24). Suppose " $<$ " holds in (5.24). Then there is a generic extension $V_{\mathrm{B}}[G]$ such that there are $B_{0}, B_{1} \in \mathcal{J}$ satisfying $\left|B_{0} \cap\left(\tau_{x}\right)_{G}\right|=\omega$ and $\left|B_{1} \cap\left(\tau_{x}\right)_{G}^{c}\right|=\omega$. Consequently $\left(\tau_{x}\right)_{G}$ is neither finite nor cofinite and neither $\left(\tau_{x}\right)_{G}$ nor $\left(\tau_{x}\right)_{G}^{c}$ kills $\mathcal{J}$. A contradiction,

Let $b=\left\|\forall B \in \check{\mathcal{J}} B C^{*} \tau_{x}\right\|$. In order to prove $x \rightarrow_{\tau_{*}} b$ we check condition (d) of Theorem 5.4.1. Let $A \in[\omega]^{\omega}$. Since $\mathcal{J}$ is a tall ideal there is $B_{0} \in[A]^{\omega} \cap \mathcal{J}$. Then by $(5,24) b \leq\left\|\tilde{B}_{0} \subset^{*} \tau_{x}\right\| \leq\left\|\left|\tilde{B}_{0} \cap \tau_{x}\right|=\check{\omega}\right\| \leq b$ which implies that $\left\|\tilde{B}_{0} \subset^{*} \tau_{x}\right\|=\left\|\left|\tilde{B}_{0} \cap \tau_{x}\right|=\omega\right\|$ and we are done.

(c) is proved in the proof of (b). 
Example 5.4.12 There exists a sequence $x$ which does not converge although $\tau_{x}$ kills some tall ideal in some extension. Let $\mathbb{B}$ be a c.B.a. which produces new reals in each extension. Then, using Lemma 4.2.1, like in the proof of Theorem 5.4.10 we find a tall ideal $\mathcal{J}$ and a sequence $y$ such that

$$
1 \Vdash \tau_{y} \in[\hat{\omega}]^{\hat{\omega}} \wedge \forall B \in \mathcal{J}\left|B \cap \tau_{y}\right|<\dot{\omega} .
$$

Let $P=\{0,2,4, \ldots\}$ and $Q=\{1,3,5, \ldots\}$. By $(5.25)\left\|\left|\tau_{y} \cap \widetilde{P}\right|=\breve{\omega}\right\|>0$ or $\left\||| \tau_{y} \cap \bar{Q} \mid=\breve{\omega}\right\|>0$. W.l.o.g. suppose the first inequality holds and let $0<p<$ $\left\|\left|\tau_{y} \cap \tilde{P}\right|=\bar{\omega}\right\|$ and $x=\left\langle y_{0} \wedge p, p^{\prime}, y_{2} \wedge p, p^{\prime}, y_{4} \wedge p, \ldots\right\rangle$. It is easy to check that $p \Vdash \tau_{x}=\tau_{y} \cap \check{P}$ and $p^{\prime} \Vdash \tau_{x}=\dot{Q}$. So, by the choice of $p$ and (5.25) we have $p \Vdash\left|\tau_{x}\right|=\breve{\omega} \wedge \forall B \in \mathcal{J}\left|B \cap \tau_{x}\right|<\breve{\omega}$, thus $\tau_{x}$ kills $\mathcal{J}$ in some extensions. On the other hand, $p<1$ implies $p^{\prime}>0$, so if $G$ is a $\mathbb{B}$-generic filter over $V$ and $p^{\prime} \in G$, then in $V_{\mathbb{B}}[G]$ we have $\left(\tau_{x}\right)_{G}=\{1,3,5, \ldots\}$. Hence $\left(\tau_{x}\right)_{G}$ is an infinite coinfinite old subset of $\omega$ which can not kill any tall ideal, and, by Theorem 5.4.11 the sequence $x$ does not converge.

According to Theorem 5.4.9 a posteriori convergence implies $a_{x}=b_{x}$ and, according to Theorem 5.4.10, $a_{x}=b_{x}$ must not imply a posteriori convergence. We will show that, for the lim sup-stable algebras, these two conditions are equivalent.

Theorem 5.4.13 Let $\mathbb{B}$ be a limsup-stable c.B.a., let $x \in \mathbb{B}^{\omega}$ and $b \in \mathbb{B}$. Then

$$
x \rightarrow \tau_{0} b \Leftrightarrow a_{x}=b_{x}=b .
$$

Proof. The direction " $\Rightarrow$ " is Theorem 5.4.9. In order to prove " $\angle$ "suppose $a_{x}=$ $b_{x}=b$. According to Theorem 5.4.1 we prove

$$
\forall A \in[\omega]^{\omega} \exists B \in[A]^{\omega}\left\|\dot{B} \cap \tau_{x} \mid=\tilde{\omega}\right\|=\left\|\dot{B} C^{*} \tau_{x}\right\|=b .
$$

Let $A \in[\omega]^{\omega}$. By Lemmas 5.2.6 and 5.2.4, the sets $D_{x}$ and $\Delta_{x}$ are dense open subsets of $[\omega]^{\omega}$, so $D_{x} \cap \Delta_{x}$ is dense in $[\omega]^{\omega}$ as well. Hence there is $B_{0} \in[A]^{\omega} \cap$ $D_{x} \cap \Delta_{x}$. Since $B_{0} \in D_{x}$, for each $B \in\left[B_{0}\right]^{\omega}$ there holds $\left\|\left|\tilde{B} \cap \tau_{x}\right|=\dot{\omega}\right\|=$ $\left\|\left|\tilde{B}_{0} \cap \tau_{x}\right|=\tilde{\omega}\right\|$, so

$$
\left\|\left|\check{B}_{\theta} \cap \tau_{x}\right|=\dot{\omega}\right\|=\bigwedge_{B \in\left[B_{0}|\mu|\right.}\left\|\left|\check{B} \cap \tau_{x}\right|=\check{\omega}\right\| \leq b_{x}=b .
$$

Since $B_{0} \in \Delta_{x}$, for each $B \in\left[B_{0}\right]^{\omega}$ there holds $\left\|\check{B} \subset^{*} \tau_{x}\right\|=\left\|\tilde{B}_{0} \subset^{*} \tau_{x}\right\|$, so

$$
\left\|\tilde{B}_{0} C^{*} \tau_{x}\right\|=\bigvee_{B \in\left[B_{0}\right]^{\omega}}\left\|\tilde{B} C^{*} \tau_{x}\right\| \geq a_{x}=b .
$$

Now, by (5.28) and (5.29), there holds $b \leq\left\|\check{B}_{0} \subset^{*} \tau_{x}\right\| \leq\left\|\left|\check{B}_{0} \cap \tau_{x}\right|=\check{\omega}\right\| \leq b$ and $(5.27)$ is proved. 
Remark 5.4.14 Let $\tilde{\lambda}: \mathbb{B}^{\omega} \rightarrow P(\mathbb{B})$ be the a priori limit operator defined by

$$
\tilde{\lambda}(x)=\left\{\begin{array}{cl}
\left\{a_{x}\right\}, & a_{x}=b_{x}, \\
\emptyset, & \text { otherwise. }
\end{array}\right.
$$

This operator generates the topology $\mathcal{O}_{\bar{\lambda}}$. By Theorem 5.4.9, for each sequence $x$ we have $\lim _{\tau_{s}}(x) \subset \vec{\lambda}(x)$, which implies $\mathcal{O}_{\hat{\lambda}} \subset \tau_{s}$. According to Theorem 5.4 .13 , if $\mathbb{B}$ satisfies $(\hbar)$ this topology is the same as $\tau_{s}$. This is not true in general. According to Example 5.4.12, there exists a Boolean algebra $\mathbb{B}$ and a sequence $x \in \mathbb{B}^{\omega}$ which does not converge a posteriori in topology $\tau_{s}$, although $a_{x}=b_{x}$. Therefore, for the Boolean algebra $\mathbb{B}$ there holds $\mathcal{O}_{\bar{\lambda}} \subsetneq \tau_{s}$.

\subsection{The sequential compactness}

In [6, Theorem 4.1] it is proved that if $\left\langle\mathbb{B}, \tau_{s}\right\rangle$ is a sequentially compact space, then $\mathbb{B}$ does not add independent reals. Also it is proved that, assuming ccc, the reverse implication is also true. This result can be extended to the following theorem.

Theorem 5.5.1 If $\mathbb{B}$ is lim sup-stable c.B.a., then $\left\langle\mathbb{B}, \tau_{s}\right\rangle$ is sequentially compact space iff $\mathbb{B}$ does not add an independent reals.

Proof: $\Leftrightarrow$ If $r \subset \omega$ is a real belonging to some generic extension $V_{\mathrm{B}}[G]$, then $r=\tau_{G}$ for some $\mathbb{B}$-name $\tau$ and if $x_{n}=\|n \in \tau\|, n \in \omega$, then the nice name $\tau_{x}$ also represents $r$. So, it is sufficient to prove that for each sequence $x \in \mathbb{B}^{\omega}$ there holds

$$
1 \Vdash \exists C \in\left(\left([\omega]^{\omega}\right)^{V}\right)^{-}\left(C \subset \tau_{x} \vee C \subset \tilde{\omega} \backslash \tau_{x}\right) .
$$

Let $x \in \mathbb{B}^{\omega}$. By the sequential compactness of $\tau_{s}$ there is $f \in \omega^{\dagger \omega}$ and $b \in \mathbb{B}$ such that $x \circ f \rightarrow \tau_{\tau_{s}} b$. By Theorem 5.4.9 we have $a_{x \circ f}=b_{x \circ f}$, which is, by Lemma 4.2.7, equivalent to $b_{x \circ f} \leq a_{x \circ f}$, and by Lemma 4.2 .4 to

$$
\begin{gathered}
\left.1 \mathbb{I} \quad \forall A \in\left(\left([\omega]^{\omega}\right)^{V}\right) \exists B \in\left((\mid A]^{\omega}\right)^{V}\right)\left|f[B] \cap \tau_{x}\right|<\dot{\omega} \vee \\
\left.\forall A \in\left(\left([\omega]^{\omega}\right)^{V}\right) \exists B \in\left((\mid A]^{\omega}\right)^{V}\right) f[B] C^{*} \tau_{x} .
\end{gathered}
$$

Now, since $f$ is " $1-1$ ". (5.31) implies (5.30).

$\Leftrightarrow$ Let $x \in \mathbb{B}^{\omega}$ be a sequence having no convergent subsequences. Like in the proof of Theorem 5.4.13, using $(\hbar)$ we find a set $A \in D_{x} \cap \Delta_{x}$. Now, if $c=\left\|\breve{A} C^{*} \tau_{x}\right\|$ and $d=\left\|\left|\tilde{A} \cap \tau_{x}\right|=\tilde{\omega}\right\|$ then

$$
\forall B \in[A]^{\omega}\left(\left\|\dot{B} C^{*} \tau_{x}\right\|=c \wedge\left\|\left|\tilde{B} \cap \tau_{x}\right|=\check{\omega}\right\|=d\right) .
$$

Suppose $c=d$. Let $f_{A}: \omega \rightarrow A$ be the increasing enumeration of the set $A$. Then, by Lemma 4.2.4 and since $f_{A}[\omega]=A$, we have $\liminf x \circ f_{A}=\limsup x \circ f_{A}$ 
thus the subsequence $x \circ f_{A}$ of the sequence $x$ converges, which contradicts the assumption, so $c<d$. According to (5.32) for each $B \in[A]^{\omega}$ there holds $d \backslash c$ It $\left|\breve{B} \cap \tau_{x}\right|=\check{\omega} \wedge\left|\breve{B} \backslash \tau_{x}\right|=\check{\omega}$ hence $d \backslash c \Vdash \forall B \in\left(\left([A]^{\omega}\right)^{V}\right)\left(\tau_{x}\right.$ splits $\left.B\right)$. Finally, let $G$ be a $\mathbb{B}$-generic filter over $V$ containing $d \backslash c$. Then in $V_{\mathbb{B}}[G]$ the real $\left(\tau_{x}\right)_{G}$ splits each infinite subset of $A$ and consequently $f_{A}^{-1}\left[\left(\tau_{x}\right)_{G}\right]$ splits each element of $[\omega]^{\omega}$ that is $f_{A}^{-1}\left[\left(\tau_{x}\right)_{G}\right]$ is an independent real.

Theorem 5.5.2 If the space $\left\langle\mathbb{B}, \tau_{s}\right\rangle$ is sequentially compact, then $\mathbb{B}$ is lim supstable algebra.

Proof. Let us suppose that $\mathbb{B}$ is not limsup-stable algebra. Then, there is a sequence $x \in \mathbb{B}^{\omega}$ such that

$$
\forall z \prec x \exists t \prec z \limsup t<\limsup z .
$$

Suppose that the space $\left\langle\mathbb{B}, \tau_{s}\right\rangle$ is sequentially compact. Then $x$ has a convergent subsequence $y \rightarrow_{\tau_{s}} b$ and, by Theorem 5.4.1(b), $y$ has a subsequence $z$ which converges to $b$ a priori, that is

$$
\liminf z=\limsup z=b .
$$

Now $z \prec y \prec x$ implies $z \prec x$ so, by (5.34) there is $t \prec z$ such that limsup $t<$ $\limsup z$. But this is impossible, since $\liminf z \leq \liminf t \leq \limsup t \leq$ $\lim \sup z$ and (5.34) holds. Thus $\left\langle\mathbb{B}, \tau_{s}\right\rangle$ is not a sequentially compact space.

Combining results from Theorems 5.5.1 and 5.5.2 we obtain

Theorem 5.5.3 Let $\mathbb{B}$ be a c.B.a. Then the space $\left\langle\mathbb{B}, \tau_{s}\right\rangle$ is sequentially compact iff $\mathbb{B}$ is limsup-stable and the forcing by $\mathbb{B}$ does not add an independent real.

Corollary 5.5.4 (a) $(\omega, 2)$-distributive c.B.a is sequentially compact iff it is 5 -cc.

(b) $P(\kappa)$ is sequentially compact iff $\kappa<\mathfrak{s}$ (see [51]).

(c) Each Suslin algebra (i.e. a non-atomic, ccc and $\omega$-distributive c.B.a.) is sequentially compact.

Proof. (a) follows from Theorems 5.5.4 and 5.3.3 and Remark 4.3.2. (b) and (c) follow from (a).

Example 5.5.5 If $t<5$, then $P(t)$ is an example of a sequentially compact space which is not $\mathrm{t}-\mathrm{cc}$. 
The examples of atomic sequentially compact c.B.a.'s are $P(\kappa)$, for $\kappa<\mathfrak{s}$. If $\mathfrak{s}=\omega_{1}$, then the only example is $P(\omega)$. If we consider non-atomic algebras we have Suslin algebras (consistent examples) which do not produce new reals. Moreover, in [6] three consistent examples of ccc (and, hence, satisfying $(\hbar)$ ) c.B.a.'s which produce new but not produce independent reals are presented. Clearly, they are sequentially compact. On the other hand, using a result of Veličković given in [54] we prove

Theorem 5.5.6 $5=\omega_{1}$ (and, in particular, $\mathrm{CH}$ ) implies $P(\omega)$ is the unique sequentially compact c.B.a. in the class of Suslin forcing notions.

Proof. Let $s=\omega_{1}$ and let $\mathbb{B}$ be a sequentially compact c.B.a. belonging to the class of Suslin forcings. Then, by Theorem 5.5.3, $(\hbar)$ holds in $\mathbb{B}$ and, by Corollary 5.3.5, $\mathbb{B}$ is ccc. Also, by Theorem 5.5.3 again, $\mathbb{B}$ does not produce independent reals by forcing. Suppose $\mathbb{B}$ in non-atomic. Then $\mathbb{B}$ is a non-atomic ccc Suslin forcing and, according to Corollary 1 and Theorem 2 of [54], $\mathbb{B}$ must produce independent reals, a contradiction. Thus $\mathbb{B}$ is an atomic ccc c.B.a. and, hence, $\mathbb{B}$ is isomorphic to $P(\omega)$.

The preceding theorem can be regarded as a partial answer to the question concerning the existence of non trivial compact spaces of the form $\left\langle\mathbb{B}, \tau_{s}\right\rangle$ asked in Remark 4.2(iii) of [6]. 
There is no branch of mathematics, however abstract, which may not someday be applied to the phenomena of the real world.

Nicolai Lobachevsky

\section{Chapter 6}

\section{The topology $\mathcal{O}^{\uparrow}$}

In this chapter is investigated the topology $\mathcal{O}^{\dagger}$, generated by the a priori limit operator $\lambda_{\text {sup }}$, defined by $\lambda_{\text {sup }}(x)=\{\lim \sup x\}$. Properties of closed sets and the closure operator are investigated and the closure of some specific sets has been determined. In cce c.B.a.'s a closed set is described by the family of its minimal elements. Some necessary conditions for a subset of $\mathbb{B}$ to be the set of minimal elements of a closed set are isolated.

In Section 6.2 we characterize the class of Boolean algebras in the equality $u=u^{2}$ holds. In Sections 6.3 and 6.4 the properties of the a posteriori limit operator are investigated, firstly in general case, and then on Boolean algebras satisfying $u=u^{2}$.

Section 6.5 characterizes the set of minimal elements of a closed set in the algebra $P(\omega)$, using the notion of subbase countable compactness.

\subsection{Closed sets and the closure operator}

\subsubsection{Generating $\mathcal{O}^{\dagger}$ by an a priori limit operator}

Let $\lambda_{\text {sup }}: \mathbb{B}^{\omega} \rightarrow P(\mathbb{B})$ be the a priori limit operator on a complete Boolean algebra $\mathbb{B}$ defined by

$$
\lambda_{\text {sup }}(x)=\{\lim \sup x\} .
$$


Obviously, the operator $\lambda_{\text {sup }}$ satisfies condition (L1).

For the sequence $x=\langle 1,0,1,0,1,0, \ldots\rangle$ in $\mathbb{B}$ and its subsequence $y$ defined by $y=\langle 0,0,0, \ldots\rangle$ we have limsup $x=1$ and limsup $y=0$, So, $\lambda_{\text {sup }}(x) \not \subset$ $\lambda_{\text {sup }}(y)$, hence $\lambda_{\text {sup }}$ does not satisfy (L2).

Therefore, we close the operator $\lambda_{\text {sup }}$ under (L2) (see Theorem 2.3.7).

Theorem 6.1.1 For each $y \in \mathbb{B}^{\omega}$ there holds

$$
\bar{\lambda}_{\text {sup }}(y)=(\limsup y) \hat{\uparrow}=\left\|\left|\tau_{y}\right|=\tilde{\omega}\right\| \uparrow .
$$

Proof. Let $y=\left\langle y_{n}: n \in \omega\right\rangle$ be a sequence in $\mathbb{B}$. The closure of $\lambda_{\text {sup }}$ under (L2), according to Theorem 2.3 .7 , is defined by

$$
\bar{\lambda}_{\text {sup }}(y)=\bigcup_{x \in \mathbb{B}^{\omega}, f \in \omega^{\top \omega}, y=x \text { of } f} \lambda_{\text {sup }}(x) .
$$

According to Theorem 4.2.2, we have that limsup $y=\left\||| \tau_{y} \mid=\check{\omega}\right\|$. Therefore, it remains to be proved that $\bar{\lambda}_{\text {sup }}(y)=(\lim \sup y) \uparrow$.

(c) Let $b \in \bar{\lambda}_{\text {sup }}(y)$. Then there exists a sequence $x$ in $\mathbb{B}$ and a function $f \in \omega^{\dagger \omega}$ such that $y=x \circ f$ and $b \in \lambda_{\text {sup }}(x)$. Therefore $b=\lim \sup x$, and, according to Corollary 3.3.7, limsup $y \leq \lim \sup x=b$, so $b \in(\lim \sup y) \uparrow$.

(ว) Let $x$ be a sequence in $\mathbb{B}$ and $b \geq \lim \sup x$. Let us prove that $b \in \bar{\lambda}_{\text {sup }}(x)$. Let us define the sequence $y$ in $\mathbb{B}$ by $y=\left\langle x_{0}, b, x_{1}, b, x_{2}, \ldots\right\rangle$. Then

$$
\begin{gathered}
\limsup y=\bigwedge_{k \in \omega} \bigvee_{n \geq k} y_{n}=\bigwedge_{k \in \omega}\left(b \vee \bigvee_{n \geq \frac{k}{2}} x_{n}\right)=b \vee \bigwedge_{k \in \omega} \bigvee_{n \geq \frac{k}{2}} x_{n}= \\
=b \vee \bigwedge_{k \in \omega} \bigvee_{n \geq k} x_{n}=b \vee \lim \sup x=b .
\end{gathered}
$$

For $f(k)=2 k$ there holds $x=y \circ f$. Hence $b \in \bar{\lambda}_{\text {sup }}(x)$.

Using this result as a motivation, instead of $\bar{\lambda}_{\text {sup }}$ we will write $\lambda^{\dagger}$. According to Theorem 2.3.10, the topology generated by the operator $\lambda_{\text {sup }}$ coincides with the topology generated by the operator $\lambda^{\dagger}$. Further on, this topology will be denoted by $\mathcal{O}^{\uparrow}$ and the corresponding family of closed sets by $\mathcal{F}^{\dagger}$.

\subsubsection{A characterization of closed sets and some properties}

As in Subsection 2.3.2, using the a priori limit operator $\lambda^{\top}$, we define the operator $u_{\lambda\urcorner}: P(\mathbb{B}) \rightarrow P(\mathbb{B})$ by

$$
u_{\lambda i}(A)=\bigcup_{y \in A^{\omega}} \cdot \lambda^{\dagger}(y)
$$

Obviously, $u_{\lambda \uparrow}=u_{\lambda \uparrow} \uparrow$. 
In this chapter, instead of $u_{\lambda \uparrow}$ we will often use the abbreviation $u$. Since $\lambda^{\dagger}$ satisfies conditions (L1) and (L2), by Theorem 2.3.13, the set $F$ is closed in the space $\left\langle\mathbb{B}, \mathcal{O}^{\top}\right\rangle$ iff $F=u(F)$.

Theorem 6.1.2 For a set $F \subset \mathbb{B}$ the following conditions are equivalent:

(a) $F$ is closed in the space $\left\langle\mathbb{B}, \mathcal{O}^{\dagger}\right\rangle$.

(b) $F$ is upward closed and closed with respect to the lim sup operator.

(c) $F$ is upward closed and for each decreasing sequence $x$ in $F$ we have $\bigwedge_{n \in \omega} x_{n} \in F\left(F\right.$ is $\omega_{1}$-closed).

Proof. (a) $\Rightarrow$ (b) Let $F$ be a closed set. Then $F=u(F)=u(F) \uparrow$, which implies that $F$ is upward closed. Theorem 2.3 .13 implies that $F$ is closed with respect to the a priori operator $\lambda^{\top}$. Since for each sequence $x, \limsup x \in \lambda^{\top}(x)$, we conclude that $F$ is closed with respect to the lim sup operator.

(b) $\Rightarrow$ (a) Let $F$ be a set closed with respect to the lim sup operator such that $F=F \uparrow$. Let us prove that $F=u(F)$. Let $x$ be a sequence in $F$. Then limsup $x \in F$ and each point $a$ such that $a \geq \lim \sup x$ is also in $F$. Therefore limsup $x \uparrow=\lambda^{\dagger}(x) \subset F$, which implies $u(F) \subset F$. The equality follows from Lemma 2.3.12.

(b) $\Rightarrow$ (c) It follows directly, since lim sup of a decreasing sequence is the infimum of elements of the sequence.

(c) $\Rightarrow$ (b) It will be sufficient to prove that $u(F)=F$. Let $b \in u(F)$. Then there exists a sequence $a=\left\langle a_{n} ; n \in \omega\right\rangle$ in $F$ such that $b \geq \limsup a$. Let

$$
c_{k}=b \vee \bigvee_{n \geq k} a_{n}, k \in \omega .
$$

Since $c_{k} \geq a_{k} \in F$, we have $c_{k} \in F \uparrow=F$, which implies that $c=\left\langle c_{k}: k \in\right.$ $\omega\rangle \in F^{\omega}$. It is obvious that the sequence $c$ is decreasing, which, by (c), implies that $\bigwedge_{k \in \omega} c_{k} \in F$. Finally, from

$$
\bigwedge_{k \in \omega} c_{k}=\bigwedge_{k \in \omega}\left(b \vee \bigvee_{n \geq k} a_{n}\right)=b \vee \bigwedge_{k \in \omega} \bigvee_{n \geq k} a_{n}=b \vee \limsup a=b
$$

it follows that $b \in F$.

Corollary 6.1.3 Each closed set in the topological space $\left\langle\mathbb{B}, \mathcal{O}^{\top}\right\rangle$ is upward closed, Each topologically open set is a downward closed (algebraically open) subset of the Boolean algebra $\mathbb{B}$. Therefore, for each point $b$ and its neighborhood $U$ we have $b \downarrow \subset U$. So, each not empty open set contains 0 .

\section{Corollary 6.1.4}

(a) The topological space $\left\langle\mathbb{B}, \mathcal{O}^{\dagger}\right\rangle$ is connected. 
(b) The topological space $\left\langle\mathbb{B}, \mathcal{O}^{\dagger}\right\rangle$ is $T_{0}$ and can not be $T_{1}$.

(c) The topological space $\left\langle\mathbb{B}, \mathcal{O}^{\top}\right\rangle$ is compact.

Proof.

(a) $\mathbb{B}$ can not be the union of two disjoint non-empty open sets, since both of them would contain 0 .

(b) Since each non-empty open set contains 0 , the space is not $T_{1}$. Since, for each $b \in \mathbb{B}$, the set $\mathbb{B} \backslash(b \uparrow)$ is open, one can easily verify that $\left\langle\mathbb{B}, \mathcal{O}^{\dagger}\right\rangle$ is a $T_{0}$ space.

(c) The statement follows directly from the fact that the only open set containing 1 is $\mathbb{B}$.

The complementation is not a continuous function in $\left\langle\mathbb{B}, \mathcal{O}^{\dagger}\right\rangle$, since the inverse image of an open set (which is also a downward closed set) is an upward closed set. For meet and join as operations in $\mathbb{B}$ we have the following.

Lemma 6.1.5 For each element $a \in \mathbb{B}$ the functions $f_{a}^{\vee}, f_{a}^{\wedge}: \mathbb{B} \rightarrow \mathbb{B}$ defined by $f_{a}^{\vee}(x)=x \vee a$ and $f_{a}^{\wedge}(x)=x \wedge a$ are continuous.

Proof. We will prove that $f_{a}^{\wedge}$ is continuous. The proof for $f_{a}^{\vee}$ is analogous. Let $F$ be a closed set. We have

$$
x \in\left(f_{a}^{\wedge}\right)^{-1}[F] \Leftrightarrow f_{a}^{\wedge}(x) \in F^{\prime} \Leftrightarrow x \wedge a \in F .
$$

So,

$$
\left(f_{a}^{\wedge}\right)^{-1}[F]=\{x \in \mathbb{B}: x \wedge a \in F\} .
$$

We will prove that $\left(f_{a}^{\wedge}\right)^{-1}[F]$ is closed, i.e., by Theorem $6.1 .2(\mathrm{c}),\left(f_{a}^{\wedge}\right)^{-1}[F]$ is upward closed and $\omega_{1}$-closed.

Firstly, let us prove that $\left(f_{a}^{\wedge}\right)^{-1}[F]$ is upward closed. Let $x_{1} \geq x \in\left(f_{a}^{\wedge}\right)^{-1}[F]$. Then $x_{1} \wedge a \geq x \wedge a \in F$, and since $F=F \dagger$, we have $x_{1} \wedge a \in F$, i.e. $x_{1} \in\left(f_{a}^{\wedge}\right)^{-1}[F]$.

It remains to be proved that $\left(f_{a}^{\wedge}\right)^{-1}[F]$ is $\omega_{1}$-closed. Let $\left\langle x_{n}: n \in \omega\right\rangle$ be a decreasing sequence in $\left(f_{a}^{\wedge}\right)^{-1}[F]$. Then $\left\langle x_{n} \wedge a: n \in \omega\right\rangle$ is a decreasing sequence in $F$, and since $F$ is $\omega_{1}$-closed, there holds $\bigwedge_{n \in \omega}\left(x_{n} \wedge a\right)=\left(\bigwedge_{n \in \omega} x_{n}\right) \wedge a \in F$. So, $\bigwedge_{n \in \omega} x_{n} \in\left(f_{a}^{\wedge}\right)^{-1}[F]$.

\subsubsection{The closure operator}

Iterating the operator $u \omega_{1}$-times, as in Theorem 2,3.14, we obtain the operator $\mathrm{cl}_{\omega_{1}}$ which is, by the same theorem, the closure operator in the space $\left\langle\mathbb{B}, \mathcal{O}^{\dagger}\right\rangle$. 
According to Lemma 2.3.12, we have $A \subset u(A) \subset \operatorname{cl}_{\alpha}(A)$, for each $A \subset \mathbb{B}$ and $1 \leq \alpha \leq \omega_{1}$. Since the operator $u$ is defined on a Boolean algebra, we can refine this sequence of inclusions.

Theorem 6.1.6 For each set $A \subset \mathbb{B}$ and $\alpha \in\left[1, \omega_{1}\right]$ we have

$$
A \subset A \uparrow \subset u(A)=u(A) \uparrow \subset \mathrm{cl}_{\alpha}(A)=\mathrm{cl}_{\alpha}(A) \uparrow \subset(\bigwedge A) \uparrow .
$$

Proof. $(A \subset A \dagger)$ Obvious.

$(A \uparrow \subset u(A))$ Let $b \in A \uparrow$. Then there exists $a \in A$ such that $a \leq b$. Since $\lambda^{\top}(\langle a\rangle)=\limsup \langle a\rangle \uparrow=a \uparrow$, we have $b \in \lambda^{\top}(\langle a\rangle) \subset u(A)$.

$(u(A)=u(A) \uparrow)$ It is obvious that $u(A) \subset u(A) \uparrow$. Let $b \in u(A) \uparrow$. Then there exists $a \in u(A)$ such that $a \leq b$ and a sequence $x$ in $A$ such that $a \in$ $\lim \sup x \uparrow$. But then there holds $b \in \lim \sup x \uparrow$, so $b \in u(A)$.

$\left(u(A) \uparrow \subset \mathrm{cl}_{\alpha}(A)\right)$ Since, for each $\alpha \leq \beta$, we have $\mathrm{cl}_{\alpha}(A) \subset \mathrm{cl}_{\beta}(A)$, it is sufficient to show that $u(A) \uparrow \subset \operatorname{cl}_{1}(A)$, which directly follows from

$$
u(A) \uparrow=u(A)=\mathrm{cl}_{1}(A) .
$$

$\left(\mathrm{cl}_{\alpha}(A)=\mathrm{cl}_{\alpha}(A) \uparrow\right.$ ) For an ordinal $\alpha=\beta+1$ we have $\mathrm{cl}_{\alpha}(A) \uparrow=\operatorname{cl}_{\beta+1}(A) \uparrow=$ $u\left(\operatorname{cl}_{\beta}(A)\right) \uparrow=u\left(\operatorname{cl}_{\beta}(A)\right)=\operatorname{cl}_{\alpha}(A)$.

If $\alpha$ is a limit ordinal, let us suppose that for each $\gamma<\alpha$ there holds $\mathrm{ch}_{\gamma}(A)=$ $\operatorname{cl}_{\gamma}(A) \uparrow$. Let $b \in \operatorname{cl}_{\alpha}(A) \uparrow$. Then there exists $a \in \operatorname{cl}_{\alpha}(A)$ such that $a \leq b$ and there exists $\delta<\alpha$ such that $a \in \operatorname{cl}_{\delta}(A)$. Since $\operatorname{cl}_{\delta}(A)=\operatorname{cl}_{\delta}(A) \dagger$ we have that $b \in \operatorname{cl}_{\delta}(A)$, which implies that $b \in \operatorname{cl}_{\alpha}(A)$.

$\left(c l_{\alpha}(A) \dagger \subset(\Lambda A) \uparrow\right)$ Let $x$ be a sequence in $A$. Then limsup $x \geq \Lambda\left\{x_{n}:\right.$ $n \in \omega\} \geq \bigwedge A$. Therefore $\lim \sup x \uparrow \subset \wedge A \uparrow$, for an arbitrary sequence $x$ in $A$. This implies that $u(A) \subset \bigwedge A \uparrow$, and then, easily, by induction we obtain that $c_{\omega_{1}}(A) \subset \bigwedge A \uparrow$.

Let us find the closures of some specitic sets.

Lemma 6.1.7 (The closure of a finite set)

(a) $\mathrm{cl}_{\omega_{1}}(\{b\})=b \uparrow$, for each $b \in \mathbb{B}$.

(b) If $|A|<\aleph_{0}$, then $\mathrm{cl}_{\omega_{1}}(A)=\bigcup_{a \in A} a \dagger=A \uparrow$.

Proof. (a) Theorem 6.1.6 implies $b \uparrow=\{b\} \uparrow \subset \mathrm{cl}_{\omega_{1}}(\{b\}) \subset \bigwedge\{b\} \uparrow=b \dagger$.

(b) The statement follows directly from condition (CO3) and (a).

\section{Lemma 6.1.8}


(a) If $\left\langle a_{n}: n \in \omega\right\rangle$ is a decreasing sequence, then $\operatorname{cl}_{\omega_{1}}\left(\left\{a_{n}: n \in \omega\right\}\right)=$ $\left(\bigwedge_{n \in \omega} a_{n}\right) \uparrow$.

(b) If $\left\langle a_{n}: n \in \omega\right\rangle$ is an increasing sequence, then $\operatorname{cl}_{\omega_{1}}\left(\left\{a_{n}: n \in \omega\right\}\right)=a_{0} \uparrow$.

(c) If $\left\{a_{n}: n \in \omega\right\}$ is a countable antichain, then $\operatorname{cl}_{\omega_{1}}\left(\left\{a_{n}: n \in \omega\right\}\right)=\mathbb{B}$.

(d) If a set $A$ contains an infinite antichain, then $\operatorname{cl}_{\omega_{1}}(A)=\mathbb{B}$.

(e) If a set $A$ is dense in an infinite c.B.a. $\mathbb{B}$, then $\operatorname{cl}_{\omega_{1}}(A)=\mathbb{B}$.

Proof.

(a) Let $\left\langle a_{n}: n \in w\right\rangle$ be a decreasing sequence. According to Lemma 6.1.7 (a) we have $c_{\omega_{1}}\left(\left\{\bigwedge_{n \in \omega} a_{n}\right\}\right)=\left\{\bigwedge_{n \in \omega} a_{n}\right\} \uparrow$, which implies that $\left\{\bigwedge_{n \in \omega} a_{n}\right\} \uparrow$ is a closed set which includes the set $\left\{a_{n}: n \in \omega\right\}$. So, $\operatorname{cl}_{\omega_{1}}\left(\left\{a_{n}: n \in\right.\right.$ $\omega\}) \subset\left(\bigwedge_{n \in \omega} a_{n}\right) \uparrow$. To prove the opposite inclusion, it will be sufficient to show that $\bigwedge_{n \in \omega} a_{n} \in u\left(\left\{a_{n}: n \in \omega\right\}\right)$. This is true, since, by Theorem 3.3.14, limsup $\left\langle a_{n}\right\rangle=\bigwedge_{n \in \omega} a_{n}$.

(b) Let $\left\langle a_{n}: n \in \omega\right\rangle$ be an increasing sequence. Then $a_{0} \uparrow \subset \mathrm{cl}_{\omega_{1}}\left(\left\{a_{n}: n \in\right.\right.$ $\omega\}) \subset \wedge\left\{a_{n}: n \in \omega\right\} \uparrow=a_{0} \uparrow$.

(c) Let $\left\{a_{n}: n \in \omega\right\}$ be a countable antichain in $\mathbb{B}$. Without lose of generality we can suppose that there holds $n \neq m$ implies $a_{n} \neq a_{m}$. Let us consider the sequence $\left\langle a_{n}: n \in \omega\right\rangle$. According to Lemma $3.3 .14(\mathrm{~g}), \lim \sup \left\langle a_{n}\right\rangle=0$. By Theorem 6.1.2, closed sets are closed with respect to the lim sup, which implies that $0 \in \operatorname{cl}_{\omega_{1}}\left(\left\{a_{n}: n \in \omega\right\}\right)$, and since, also by Theorem 6.1.2, closed sets are upward closed, we have $0 \uparrow \subset \operatorname{cl}_{\omega_{1}}\left(\left\{a_{n} ; n \in \omega\right\}\right)$, i.e. $\mathbb{B}=\operatorname{cl}_{\omega_{1}}\left(\left\{a_{n}: n \in \omega\right\}\right)$.

(d) The statement is a direct consequence of (c).

(e) The fact that each dense set in an infinite Boolean algebra contains an infinite antichain together with (d) completes the proof.

In the following examples we will show that some inclusions from Theorem 6.1.6 can be strict.

Example 6.1.9 There exists a set $A$ such that $c_{\omega_{1}}(A) \neq(\bigwedge A) \uparrow$. Namely, let $a$ and $b$ be two incomparable elements. Then, according to Lemma 6.1.7, $\operatorname{cl}_{\omega_{1}}(\{a, b\})=(a \uparrow) \cup(b \uparrow) \neq(a \wedge b) \uparrow$.

Example 6.1.10 There exists a set $A$ such that $A \uparrow \neq u(A)$. Let $\left\langle a_{n}: n \in \omega\right\rangle$ be a strictly decreasing sequence. Then, by Lemma 3.3.14 (e), we have limsup $\left\langle a_{n}\right\rangle=$ $\bigwedge a_{n}$. So.

$$
\bigwedge a_{n}=\lim \sup \left(a_{n}\right\rangle \in \lambda^{\top}\left(\left\langle a_{n}\right\rangle\right) \subset u\left(\left\{a_{n}: n \in \omega\right\}\right),
$$

but $\bigwedge a_{n} \notin\left\{a_{n}: n \in \omega\right\} \uparrow$. 


\subsubsection{Operator Dec}

The closure operator $\mathrm{cl}_{\omega_{1}}$ is obtained by iterating the operator $u \omega_{1}$-times. For an arbitrary set $A, u(A)$ is the union of all $\lambda^{\dagger}$-limits of sequences from $A$. But, instead of using the operator $u$ for generating the closure operator, we can use another operator which concerns only decreasing sequences $\left(\left\langle y_{n}: n \in \omega\right\rangle\right)$ is a decreasing sequence iff $y_{n} \geq y_{m}$ for $n \leq m$ ).

Definition 6.1.11 Let Dec $: P(\mathbb{B}) \rightarrow P(\mathbb{B})$ be the operator defined by

$$
\operatorname{Dec}(A)=\left\{\bigwedge_{n \in \mathcal{W}} y_{n}:\left\langle y_{n}\right\rangle \text { is a decreasing sequence in } A\right\} \text {. }
$$

Lemma 6.1.12 For an arbitrary $A \subset \mathbb{B}$ we have

(a) $u(A)=\operatorname{Dec}(A \uparrow)$;

(b) $u^{2}(A)=\operatorname{Dec}(\operatorname{Dec}((A \uparrow))$;

(c) $\operatorname{Dec}(A \uparrow)=\operatorname{Dec}(A \uparrow) \uparrow$.

Proof.

(a) (C) Let $b \in u(A)$. Then there exists a sequence $a=\left\langle a_{n}: n \in \omega\right\rangle$ in $A$ such that $b \geq \lim \sup a$. The sequence $\left\langle c_{k}: k \in \omega\right\rangle$ defined by

$$
c_{k}=b \vee \bigvee_{n \geq k} a_{n}, k \in \omega
$$

is a decreasing sequence, and since $c_{k} \geq a_{k}$ we have that $\left\langle c_{k}: k \in \omega\right\rangle$ is a sequence in $A \uparrow$. From

$$
\bigwedge_{k \in \omega} c_{k}=\bigwedge_{k \in \omega}\left(b \vee \bigvee_{n \geq k} a_{n}\right)=b \vee \bigwedge_{k \in \omega} \bigvee_{n \geq k} a_{n}=b \vee \lim \sup a=b
$$

it follows that $b \in \operatorname{Dec}(A \uparrow)$.

(J) Let $b \in \operatorname{Dec}(A \uparrow)$. Then there exists a decreasing sequence $y=\left\langle y_{n}: n \in\right.$ $\omega)$ in $A \uparrow$ such that $b=\bigwedge_{n \in \omega} y_{n}$. For each $n \in \omega$ we have $y_{n} \in A \uparrow$ and therefore there exists $x_{n} \in A$ such that $y_{n} \geq x_{n}$. Hence,

$$
b=\bigwedge_{n \in \omega} y_{n}=\limsup y \geq \lim \sup x ;
$$

where $x=\left\langle x_{n}: n \in \omega\right\rangle$. This implies that $b \in(\lim \sup x) \uparrow \subset u(A) \uparrow$. Since, according to Theorem 6.1.6, $u(A)=u(A) \uparrow$, we have $b \in u(A) \uparrow$.

(b) $u(u(A))=\operatorname{Dec}(u(A) \dagger)=\operatorname{Dec}(u(A))=\operatorname{Dec}(\operatorname{Dec}(A \uparrow))$.

(c) $\operatorname{Dec}(A \uparrow)=u(A)=u(A) \uparrow=\operatorname{Dec}(A \uparrow) \uparrow$.

In the sequel we will show that iterating the operator Dec $\omega_{1}$-times we obtain the closure operator $\mathrm{cl}_{\omega_{1}}$. 
Definition 6.1.13 Let $\operatorname{Dec}_{\alpha}: P(\mathbb{B}) \rightarrow P(\mathbb{B})$, for $0<\alpha \leq \omega_{1}$, be defined by

- $\operatorname{Dec}_{1}(A)=\operatorname{Dec}(A \uparrow)$;

- $\operatorname{Dec}_{\alpha+1}(A)=\operatorname{Dec}\left(\operatorname{Dec}_{\alpha}(A)\right)$;

- $\operatorname{Dec}_{\gamma}(A)=\bigcup_{\alpha<\gamma} \operatorname{Dec}_{\alpha}(A)$, for a limit ordinal $\gamma$.

Theorem 6.1.14 For each $\alpha \in\left[1, \omega_{1}\right]$ and $A \subset \mathbb{B}$ we have $\operatorname{Dec}_{\alpha}(A)=\operatorname{cl}_{\alpha}(A)$.

Proof. Let $A \subset \mathbb{B}$

For $\alpha=1$ there holds $\operatorname{Dec}_{1}(A)=\operatorname{Dec}(A \dagger)=u(A)=\operatorname{cl}_{1}(A)$. Let us suppose that for each $\alpha \in[1, \beta)$ we have $\operatorname{Dec}_{\alpha}(A)=c l_{\alpha}(A)$. Let us prove that $\operatorname{Dec}_{\beta}(A)=\operatorname{cl}_{\beta}(A)$.

If $\beta=\gamma+1$ then $\operatorname{Dec}_{\beta}(A)=\operatorname{Dec}_{\gamma+1}(A)=\operatorname{Dec}\left(\operatorname{Dec}_{\gamma}(A)\right)=\operatorname{Dec}\left(\operatorname{cl}_{\gamma}(A)\right)=$ $\operatorname{Dec}\left(\operatorname{cl}_{\gamma}(A) \uparrow\right)=u(\operatorname{cl}(A))=\operatorname{cl}_{\gamma+1}(A)=\operatorname{cl}_{\beta}(A)$.

If $\beta$ is a limit ordinal, then $\operatorname{Dec}_{\beta}(A)=\bigcup_{\alpha<\beta} \operatorname{Dec}_{\alpha}(A)=\bigcup_{\alpha<\beta} \operatorname{cl}_{\alpha}(A)=$ $\operatorname{cl}_{\beta}(A)$.

Instead of iterating the operator $u \omega_{1}$-times, Theorem 6.1.14 ensures us that the closure of a set $A$ can be obtained iterating a simpler operator Dec, starting with the set $A \uparrow$.

\subsubsection{Closed sets and their minimal elements}

In the space $\left\langle\mathbb{B}, \mathcal{O}^{\dagger}\right\rangle$ a set $F$ is closed iff $u(F)=F$. But this characterization holds in each space obtained by an a priori limit operator satisfying (L1) and (L2). In this subsection we will give some representations of closed sets in the language of Boolean algebra. First we will consider closed sets in ccc Boolean algebras.

Theorem 6.1.15 Let $\mathbb{B}$ be a ccc c.B.a. Then each closed set $F \in \mathcal{F}^{\top}$ can be represented as

$$
F=\bigcup_{b \in \operatorname{Min}(F)}(b \uparrow)_{t}
$$

where $\operatorname{Min}(F)=\{b \in F: b$ is a minimal element of $F\}$.

Proof. Let $F \subset \mathbb{B}$ such that $u(F)=F$ and let $a \in F$. Let us suppose that there does not exist a minimal element of $E$ less than equal to $a$, i.e.

$$
\forall c \in F \cap a \downarrow \exists d \in F d<c .
$$

Then it is clear that $0 \notin F$. We recursively define a chain $\left\langle a_{\alpha}: \alpha<\omega_{1}\right\rangle$ such that $a_{\alpha} \in F \cap a \downarrow$ and $a_{\beta}<a_{\alpha}$ for $\alpha<\beta$.

Let $a_{0}=a$. Let us suppose that $a_{\gamma}$ is defined for each $\gamma<\beta$. If $\beta=\alpha+1$, then $a_{\alpha} \in F \cap a \downarrow$ and, by (6.1), there exists $a_{\alpha+1} \in F$ such that $a_{\alpha+1}<a_{\alpha}$. 
If $\beta$ is a limit ordinal, since $\beta<\omega_{1}$, then there exists an increasing sequence of ordinals $\left\langle\alpha_{n}: n \in \omega\right\rangle$ such that $\sup \alpha_{n}=\beta$. Then $\left\langle a_{\alpha_{n}}: n \in \omega\right\rangle$ is a strictly decreasing sequence in $F$. According to Theorem 6.1.2 (c), $\bigwedge_{n \in \omega} a_{\alpha_{n}} \in F$. For let $a_{\beta}=\bigwedge_{n \in \omega} a_{\alpha_{n}}$ we have $a_{\beta}<a_{\alpha}$, for each $\alpha<\beta$,

In this way we obtained a chain of size $\omega_{1}$, which generates the antichain $\left\langle a_{\alpha}\right\rangle$ $\left.a_{\alpha+1} \vdots \alpha<\omega_{1}\right)$ of size $\omega_{1}$. This contradicts the fact that $\mathbb{B}$ is a ccc algebra.

Since $F$ is an upward closed set, we have $F=\bigcup_{x \in F} x \uparrow$. Also, for each $a \in F$ there exists $c \in \operatorname{Min}(F)$ such that $c \leq a$ which implies $a \uparrow \subset c \uparrow$. So, $F=\bigcup_{a \in F} a \uparrow \subset \bigcup_{c \in \operatorname{Min}(F)} c \uparrow \subset F$.

In general, closed sets in $\left\langle\mathbb{B}, \mathcal{O}^{\top}\right\rangle$ must not bave minimal elements.

Example 6.1.16 Let $\mathbb{B}$ be a c.B.a. which is not ccc. Then in $\mathbb{B}$ there exists an uncountable strictly decreasing chain $\left\langle a_{\alpha}: \alpha<\omega_{1}\right\rangle$. Let $A=\left\{a_{\alpha}: \alpha<\omega_{1}\right\}$. Let us show that $A \uparrow=\bigcup_{\alpha<\omega_{1}} a_{\alpha} \uparrow$ is a closed set. By Lemma 6.1 .8 (a), it will be sufficient to prove that $\operatorname{Dec}(A \uparrow)=A \uparrow$. Obviously $A \uparrow \subset \operatorname{Dec}(A \uparrow)$. Let $b \in \operatorname{Dec}(A \uparrow)$. Then there exists a decreasing sequence $\left\langle b^{n}: n \in \omega\right\rangle$ in $A \uparrow$ such that $b=\bigwedge_{n \in \omega} b^{n}$. For each $n \in \omega$ there exists $a^{n}$ in $A$ such that $b^{n} \geq a^{n}$. We can w.l.o.g. suppose that $\left\langle a^{n}: n \in \omega\right\rangle$ is also a decreasing sequence. Since the cofinality of $\omega_{1}$ is not $\omega$, there exists $\alpha<\omega_{1}$ such that $a_{\alpha}<a^{n}$ for each $n \in \omega$, which implies that

$$
b=\bigwedge_{n \in \omega} b^{n} \geq \bigwedge_{n \in \omega} a^{n} \geq a_{\alpha} .
$$

So, $b \in a_{\alpha} \uparrow \subset A \uparrow$.

Since $\bigwedge A \notin A \uparrow$ we conclude that $A \uparrow$ is a closed set without minimal elements.

In Theorem 6.1.15 it is shown that each closed set in a ccc c.B.a. is determined by its minimal elements. In the following theorem we will consider a set of the form $F=\bigcup_{x \in X} q_{x} \uparrow \subset \mathbb{B}$, where $X$ is an arbitrary set and $\mathbb{B}$ an arbitrary Boolean algebra. We will examine when the set $\left\{q_{x}: x \in X\right\}$ is the set of minimal elements of $F$ and, using the set $\left\{q_{x} \leqslant x \in X\right\}$, we will give a characterization of closed sets of the given form.

Theorem 6.1.17 Let $\mathbb{B}$ be a c.B.a, $X$ a non empty set and $\left\{q_{x} \neq x \in X\right\} \subset \mathbb{B}$. Let $\tau=\left\{\left\langle\tilde{x}, q_{x}\right\rangle: x \in X\right\}$ and $F=\bigcup_{x \in X} q_{x} \uparrow$. Then:

(1) If $x \neq y \Rightarrow q_{x} \neq q_{y}$, then the following conditions are equivalent:

(a) $q_{x}$ and $q_{y}$ are incomparable for different $x, y \in X$;

(b) $\forall x, y \in X(x \neq y \Rightarrow \| \bar{x} \in \tau \not \supset y ̆ y>0)$;

(c) $\left\{q_{x}: x \in X\right\}=\operatorname{Min}(F)$. 
(2) The following conditions are equivalent:

(a) $F$ is closed;

(b) $u(F)=F$;

(c) $\forall f: \omega \rightarrow X \exists x \in X q_{x} \leq \limsup \left\langle q_{f(n)}\right\rangle$;

(d) $\forall f \neq \omega \rightarrow X$, where $f$ is finite to one, $\exists x \in X q_{x} \leq \limsup \left\langle q_{f(n)}\right)$;

(e) $\forall A \in[X]^{\omega} \exists x \in X 1 \Vdash \breve{x} \in \tau \Rightarrow|\tau \cap \tilde{A}|=\bar{\omega}$.

Proof. (1)

(a) $\Rightarrow$ (b) Let $x, y \in X$ such that $x \neq y$. Since (a) holds, we have $q_{x} \notin q_{y}$, which implies that $c=q_{x} \wedge q_{y}^{\prime}>0$. From $c=\|\breve{x} \in \tau\| \wedge\|\breve{y} \in \tau\|^{\prime}=\|\breve{x} \in \tau \not \supset \breve{y}\|$ it follows (b).

(b) $\Rightarrow$ (a) Let $x, y \in X$ such that $x \neq y$. Let $c=\|\tilde{x} \in \tau \not \supset \ddot{y}\|$. By (b), we have that $c=q_{x} \wedge q_{y}^{\prime}>0$, so $q_{x} \notin q_{y}$. From $d=\|\check{y} \in \tau \not \supset \breve{x}\|>0$ it follows analogously that $q_{y} \leq q_{x}$.

(a) $\Rightarrow$ (c) ( $\subset$ ) Let $x \in X$. Then $q_{x} \in F$. Let us suppose that there exists $c \in F$ such that $c<q_{x}$. Then, there exists $y \in X$ such that $q_{y} \leq c$, which implies that $q_{y}<q_{x}$. A contradiction.

(つ) Let $a$ be a minimal element of $F$. Then $a \in F$. So, there exists $x \in X$ such that $q_{x} \leq a$. Since $q_{x} \in F$ and $a$ is a minimal element, we have $a=q_{x}$.

(c) $\Rightarrow$ (a) Let $x \neq y$. Then $q_{x}$ and $q_{y}$ are two minimal elements, which implies that they are incomparable.

(2)

(a) $\Leftrightarrow$ (b) This equivalence follows from Theorem 2.3.13.

(b) $\Rightarrow$ (c) Let $f: \omega \rightarrow X$. Then $\left\langle q_{f(n)}: n \in \omega\right\rangle$ is sequence in $F$, so, $\limsup \left\langle q_{f(n)}\right\rangle \in F$. Since, $F=\bigcup_{x \in X} q_{x} \uparrow$, there exists $x \in X$ such that $q_{x} \leq \limsup \left\langle q_{f(n)}\right\rangle$.

(c) $\Rightarrow$ (d) It is obvious.

(d) $\Rightarrow$ (b) Let $a=\left\langle a_{n}: n \in \omega\right\rangle$ be a sequence in $F$. For each $n \in \omega$, there exists $x_{n} \in X$ such that $q_{x_{n}} \leq a_{n}$. Then $\lim \sup \left\langle q_{x_{n}}\right\rangle \leq \lim \sup \left\langle a_{n}\right\rangle$. Since $F$ is upward closed, it is sufficient to show that $\lim \sup \left\langle q_{x_{n}}\right\rangle \in F$. Let $f: \omega \rightarrow X$ such that $f(n)=x_{n}$. If $f$ is 'finite to one', by (d), there exists $x \in X$ such that $q_{x} \leq \limsup \left\langle q_{f(n)}\right\rangle=\limsup \left\langle q_{x_{n}}\right\rangle$. Therefore, $\limsup \left\langle q_{x_{n}}\right\rangle \in F$. If $f$ is not 'finite to one', then there exists $x \in X$ such that $x_{n}=x$ for intinitely many $n \in \omega$. Therefore $\lim \sup \left\langle q_{x_{n}}\right\rangle=\bigwedge_{k \in \omega} \bigvee_{n \geq k} q_{x_{n}} \geq \bigwedge_{k \in \omega} q_{x}=q_{x}$, which implies that $\limsup \left\langle q_{x_{n}}\right\rangle \in F$.

(d) $\Rightarrow(\mathrm{e})$ Let $A \in[X]^{\omega}$. Then there exists an injection $f: \omega \rightarrow X$ such that $f[\omega]=A$. By (d), there exists $x \in X$ such that

$$
q_{x} \leq \limsup \left\langle q_{f(n)}\right\rangle .
$$


It is clear that $q_{x}=\|\tilde{x} \in \tau\|$. Since $f$ is an injection, we have

$$
\begin{aligned}
\limsup \left\langle q_{f(n)}\right\rangle & =\bigwedge_{k \in \omega} \bigvee_{n \geq k}\|f(n) \in \tau\| \\
& =\left\|\forall k \in \bar{\omega} \exists n \geq k f(n)^{\dot{y}} \in \tau\right\| \\
& =\|\tau \cap \bar{A} \mid=\bar{\omega}\| .
\end{aligned}
$$

From (6.2) we have $\|\tilde{x} \in \tau\| \leq\||\tau \cap \breve{A}|=\check{\omega}\|$, which is equivalent to $\| \tilde{x} \in \tau \Rightarrow$ $|\tau \cap \tilde{A}|=\vec{\omega} \|=1$.

(e) $\Rightarrow$ (d) Let $f: \omega \rightarrow X$ be a 'finite to one' mapping. Then $f[\omega] \in[X]^{\omega}$, so, from (e) it follows that there exists $x \in X$ such that $\|\check{x} \in \tau\| \leq\|\tau \cap f[\omega]\|=\check{\omega}$. In (d) $\Rightarrow$ (e) part of the proof was showed that $\||\tau \cap f[\omega]|=\dot{\omega}\|=\lim \sup \left\langle q_{f(n)}\right\rangle$. This and the fact that $q_{x}=\|\tilde{x} \in \mathcal{\tau}\|$ gives (d).

In the following theorem we give a forcing characterization of closed sets in the space $\left\langle\mathbb{B}, \mathcal{O}^{\uparrow}\right\rangle$, where $\mathbb{B}$ is an arbitrary c.B.a.

Theorem 6.1.18 Let $F$ be an upward closed set in $\mathbb{B}$. Then the following conditions are equivalent:

(a) $F$ is closed;

(b) $\forall A \in[F]^{\omega} \exists a \in F a \Vdash|\Gamma \cap \bar{A}|=\tilde{\omega}$, where $\Gamma=\left\{\langle\check{b}, b\rangle: b \in \mathbb{B}^{+}\right\}$.

Proof. Since $F$ is upward closed, we have $F=\bigcup_{b \in F} b \uparrow$. By Theorem 6.1.17 (2) (a) $\Leftrightarrow(\mathrm{e})$, we have that $F$ is closed iff $\forall A \in[F]^{\omega} \exists a \in F \| \check{a} \in \tau \Rightarrow|\tau \cap \breve{A}|=$ $\bar{\omega} \|=1$, where $\tau=\{\langle\tilde{b}, b\rangle: b \in F\}$.

Firstly we will prove that

$$
1 \Vdash \tau=\Gamma \cap \tilde{F} \text {, where } \tilde{F}=\{\langle\bar{b}, 1\rangle \neq b \in F\} .
$$

Let $b \in \mathbb{B}^{+}$. If $b \in F$, then $\|\check{b} \in \tau\|=b$, and if $b \notin F$, then $\|\check{b} \in \tau\|=0$. Also, if $b \in F$, then $\|\vec{b} \in \tilde{F}\|=1$, and if $b \notin F$, then $\|\vec{b} \in \tilde{F}\|=0$. Therefore

$$
\|\tilde{b} \in \tau\|=b \wedge\|\tilde{b} \in \tilde{F}\|=\|\dot{b} \in \Gamma\| \wedge\|\tilde{b} \in \dot{F}\|=\|\tilde{b} \in \Gamma \cap \tilde{F}\| .
$$

This implies that

$$
\forall b \in \mathbb{B}^{+}\|\tilde{b} \in \tau\|=\|\tilde{b} \in \Gamma \cap \tilde{F}\|,
$$

and by Fact 4.1 .2 (b) it follows that

$$
\forall b \in \mathbb{B}^{+}\|\check{b} \in \tau \Leftrightarrow \check{b} \in \Gamma \cap \check{F}\|=1,
$$

which is equivalent to

$$
\left\|\forall b \in \check{\mathbb{B}}^{+} \check{b} \in \tau \Leftrightarrow \tilde{b} \in \Gamma \cap \tilde{F}\right\|=1 \text {, i.e. }
$$




$$
\|\tau=\Gamma \cap \tilde{F}\|=1,
$$

which completes the proof of (6.3).

Now, by $(6.3)$, there holds

$$
\forall A \in[F]^{\omega} \exists a \in F\|\tilde{a} \in \Gamma \cap \check{F} \Rightarrow|\Gamma \cap \check{F} \cap \tilde{A}|=\tilde{\omega}\|=1 .
$$

Since, for each $\mathbb{B}$-generic filter $G$ over $V$ we have $\Gamma_{G}=G$, and $F_{G}=F$, then $\|\check{a} \in \Gamma \cap \tilde{F} \Rightarrow|\Gamma \cap \tilde{F} \cap \tilde{A}|=\breve{\omega}\|=1$ is equivalent to the sentence that for each generic filter $G$ there holds $(a \in G \cap F \Rightarrow|G \cap F \cap A|=\omega$ ). Since $a \in F$ and $A \subset F$, this is equivalent to the sentence that for each $\mathbb{B}$-generic filter $G$ there holds $(a \in G \Rightarrow|G \cap A|=\omega)$, which is equivalent to $a \Vdash|\Gamma \cap \tilde{A}|=\tilde{\omega}$.

\subsection{The equality $u=u^{2}$}

A topological space of the form $\left\langle\mathbb{B}, \mathcal{O}^{\dagger}\right\rangle$ is defined by the a priori limit operator $\lambda^{\dagger}$. It is, according to Theorem 2.3.15, a sequential space. The question is when it is a Fréchet space. By Theorem 2.3.18 the equality $u=u^{2}$ implies that the space $\left\langle\mathbb{B}, \mathcal{O}^{\top}\right\rangle$ is Fréchet. Also it implies that the operator $u$ is the closure operator in the space $\left\langle\mathbb{B}, \mathcal{O}^{\dagger}\right\rangle$. Therefore, this equality will be firstly considered.

This problem is closely related to matrices. So, if $\left[a_{n k}\right]$ is a matrix in $\mathbb{B}$, then by $A$ we will denote the set $\left\{a_{n k}: n, k \in \omega\right\}$.

Theorem 6.2.1 The following conditions are equivalent:

(a) $u=u^{2}$;

(b) For each matrix $\left[a_{n k}\right]$, sequence $\left\langle y_{n}\right\rangle$ and element $b$ if $\limsup \sup _{k}\left\langle a_{n k}\right\rangle \leq$ $y_{n}$ and $\lim \sup \left\langle y_{n}\right\rangle \leq b$, for each $n \in \omega$, then there exists $x \in A^{\omega}$ such that $\lim \sup x \leq b$;

(c) For each matrix $\left[a_{n k}\right]$ and sequence $\left\langle y_{n}\right\rangle$ if $\lim \sup _{k}\left\langle a_{n k}\right\rangle \leq y_{n}$ and $\limsup \left\langle y_{n_{1}}\right\rangle=0$, for each $n \in \omega$, then there exists $x \in A^{\omega}$ such that $\lim \sup x=$ 0 ;

(d) For each matrix $\left[a_{n k}\right]$ if $\lim \sup _{n} \lim \sup _{k}\left\langle a_{n k}\right\rangle=0$ then there exists $x \in$ $A^{\omega}$ such that $\lim \sup x=0$

(e) For each matrix $\left[a_{n k}\right]$ such that the sequence $\left\langle a_{n k}: k \in \omega\right\rangle$ is decreasing for each $n$ if $\limsup _{n} \limsup \sup _{k}\left\langle a_{n k}\right\rangle=0$ then there exists $x \in A^{\omega}$ such that $\lim \sup x=0$;

(f) For each nice name $\tau$ for a subset of $\omega^{2}$, such that $\|\langle n, k\rangle \in \tau\|>0$, for each $n, k \in \omega$, and $1 \mathbb{t}$ " $\tau \cap(\{n\} \times \omega)$ is finite for almost each $n$ ", there exists an infinite set $S \subset \omega^{2}$ such that $1 \Vdash \tau \cap \tilde{S}$ is finite. 
Proof. $(\mathrm{a}) \Rightarrow$ (b) Let $\left[a_{n k}\right]$ be a matrix in $\mathbb{B}$. Let $y_{n} \geq \lim \sup \left\langle a_{n k}: k \in \omega\right\rangle$, i.e. $y_{n} \in \lambda^{\top}\left(\left\langle a_{n k}: k \in \omega\right\rangle\right)$ and $b \geq \limsup \left\langle y_{n}\right\rangle$, i.e. $b \in \lambda^{\top}\left(\left\langle y_{n}\right\rangle\right)$. Thus $b \in u(u(A))$. From (a) it follows $u(A)=u(u(A))$, so there exists a sequence $x$ in $A$ such that $b \in \lambda^{\top}(x)$, i.e. $b \geq \limsup x$.

(b) $\Rightarrow$ (a) Let $X \subset \mathbb{B}$ and $b \in u^{2}(X)$. Then there exists a sequence $y=\left\langle y_{n}\right.$ : $n \in \omega)$ in $u(X)$ such that $b \in \lambda^{\top}(y)$, i.e. limsup $y \leq b$. Since each $y_{n} \in u(A)$, there exist sequences $\left\langle a_{n k}: k \in \omega\right\rangle$ in $X$ such that $y_{n} \in \lambda^{\top}\left(\left\langle a_{n k}: k \in \omega\right\rangle\right)$, i.e. $y_{n} \geq \limsup \left\langle a_{n k}: k \in \omega\right\rangle$. Then (b) implies that there exists a sequence $x \in A^{\omega}$ such that $b \geq \limsup x$. So $b \in u(A) \subset u(X)$.

(b) $\Rightarrow$ (c) It follows directly.

(c) $\Rightarrow$ (d) It follows directly from (c) for $y_{n}=\lim \sup \left\langle a_{n k}: k \in \omega\right\rangle$.

(d) $\Rightarrow$ (e) It follows directly.

(e) $\Rightarrow$ (b) Let $\left[a_{n k}\right]$ be a matrix, $\left\langle y_{n}\right\rangle$ a sequence in $\mathbb{B}$ and $b \in \mathbb{B}$ such that $\limsup \sup _{k}\left\langle a_{n k}\right\rangle \leq y_{n}$ for each $n \in \omega$ and $\limsup \left\langle y_{n}\right\rangle \leq b$. Let $c=\limsup \left\langle y_{n}\right\rangle$. Let us define $\tilde{a}_{n k}=a_{n k} \wedge c^{\prime}$ and $\tilde{y}_{n}=y_{n} \wedge c^{\prime}$. Let $b_{n k}=V_{l \geq k} \hat{a}_{n l}$. Obviously. $\left\langle b_{n k}: k \in \omega\right\rangle$ is a decreasing sequence. Let us notice that limsup $\sup _{k}\left\langle b_{n k}\right\rangle=$ $\lim \sup _{k}\left\langle\hat{a}_{n k}\right\rangle$. We have

$$
\begin{aligned}
\limsup \limsup _{k}\left\langle b_{n k}\right\rangle & \left.=\limsup \limsup \sup _{n k}\right\rangle \\
& =\limsup \tilde{a}_{n} \limsup \sup _{k}\left\langle a_{n k} \wedge c^{\prime}\right\rangle \\
& =c^{\prime} \wedge \lim \sup _{n} \limsup \sup _{k}\left\langle a_{n k}\right\rangle \\
& \leq c^{\prime} \wedge \limsup \left\langle y_{n}\right\rangle \\
& =c^{\prime} \wedge c=0 .
\end{aligned}
$$

Therefore there exists a sequence $x=\left\langle x_{l} ; l \in \omega\right\rangle$ in $\left\{b_{n k} ; n, k \in \omega\right\}$ such that $\limsup x=0$. For each $l \in \omega$ there exist $n_{l}$ and $k_{l}$ such that $x_{l}=b_{n l}$. So, $x_{l}=\bigvee_{s \geq k_{l}} \tilde{a}_{n_{l} s} \geq \tilde{a}_{n_{l} k_{l}}=c^{\prime} \wedge a_{n_{l} k_{l}}$. Therefore, limsup $\left\langle c^{\prime} \wedge a_{n_{l} k_{l}}\right\rangle=0$, which implies limsup $\left\langle a_{n_{l} k_{l}}\right\rangle \leq c=\lim \sup \left\langle y_{n}\right\rangle \leq b$.

(f) $\Rightarrow$ (e) Let $\left[a_{n k}\right]$ be a decreasing matrix such that $\lim \sup _{n} \lim \sup _{k}\left\langle a_{n k}\right\rangle=$ 0 . If there exists $a_{n_{0} k_{0}}=0$, then, since the matrix is decreasing, we have $a_{n_{0} k}=0$ for each $k \geq k_{0}$ and therefore $\lim \sup _{k} a_{n_{0} k}=0$. So we can suppose that $a_{n k} \neq 0$ for each $n, k \in \omega$. Let $\tau=\left\{\left\langle\langle n, k\rangle^{-}, a_{n k}\right\rangle: n, k \in \omega\right\}$ be the nice name for a subset of $\omega^{2}$. Since the inequality $\|\langle n, k\rangle \in \tau\|>0$ is equivalent to $a_{n k}>0$, the first condition of $(f)$ is fulfilled. Secondly, we have

$$
\begin{aligned}
& 1 \Vdash \exists m \in \omega \forall n \geq m|\tau \cap(\{n\} \times \bar{\omega})|<\bar{\omega} \\
& \Leftrightarrow\|\exists m \in \bar{\omega} \forall n \geq m|\tau \cap(\{n\} \times \bar{\omega})|<\bar{\omega}\|=1 \\
& \Leftrightarrow\|\forall m \in \bar{\omega} \exists n \geq m|\tau \cap(\{n\} \times \bar{\omega})|=\omega\|^{\prime}=1 \\
& \Leftrightarrow\|\forall m \in \bar{\omega} \exists n \geq m|\tau \cap(\{n\} \times \bar{\omega})|=\omega\|=0
\end{aligned}
$$




$$
\begin{aligned}
& \Leftrightarrow \bigwedge_{m \in \omega} \bigvee_{n \geq m}\|\forall l \in \tilde{\omega} \exists k \geq l\langle n, k\rangle \in \tau\|=0 \\
& \Leftrightarrow \bigwedge_{m \in \omega} \bigvee_{n \geq m} \bigwedge_{l \in \omega} \bigvee_{k \geq l}\|\langle n, k\rangle \in \tau\|=0 \\
& \Leftrightarrow \bigwedge_{m \in \omega} \bigvee_{n \geq m} \bigwedge_{l \in \omega} \bigvee_{k \geq l} a_{n k}=0 \\
& \Leftrightarrow \limsup _{n} \limsup \left\langle a_{n k}\right\rangle=0,
\end{aligned}
$$

and, therefore, the second condition of $(f)$ is fulfilled. So, there exists an infinite set $S \subset \omega^{2}$ such that $1 \Vdash|\tau \cap \tilde{S}|<\tilde{\omega}$. Let $S=\left\{\left\langle s_{n}^{1}, s_{n}^{2}\right\rangle: n \in \omega\right\}$. Then

$$
\begin{aligned}
& 1 \Vdash|\tau \cap \tilde{S}|<\bar{\omega} \\
& \quad \Leftrightarrow\||\tau \cap \bar{S}|<\omega\|=1 \\
& \quad \Leftrightarrow\||\tau \cap \dot{S}|=\bar{\omega}\|=0 \\
& \quad \Leftrightarrow\left\|\forall m \in \tilde{\omega} \exists n \geq m\left\langle s_{n}^{1}, s_{n}^{2}\right\rangle \in \tau\right\|=0 \\
& \quad \Leftrightarrow \bigwedge_{n \in \omega} \bigvee_{n \geq m} a_{s_{n}^{1} s_{n}^{2}}=0 \\
& \quad \Leftrightarrow \limsup \left\langle a_{s_{n}^{1} s_{n}^{2}}\right\rangle=0 .
\end{aligned}
$$

(d) $\Rightarrow$ (f) Let $\tau=\left\{\left\langle\langle n, k\rangle, a_{n k}\right\rangle: n, k \in \omega\right\}$ be the nice name for a subset of $\omega^{2}$, where $\left[a_{n k}\right]$ is a matrix with non-zero elements (which is equivalent to $\|\langle n, k\rangle \in$ $\tau \|>0$ ). Also, from (6.4) it follows that $\limsup \operatorname{simsup}_{k}\left\langle a_{n k}\right\rangle=0$. So, there exists a sequence $x$ in $A$ such that $\lim \sup x=0$. Let $S=\left\{x_{n}: n \in \omega\right\}$. Let us notice that there does not exist $b \in \mathbb{B}$ such that $x_{n}=b$ for infinitely many $n \in \omega$, since if such $b$ would exist, then $\lim \sup x \geq b>0$. So $x$ is 'finite to one' mapping from $\omega$ onto $S$. One can easily construct a subsequence $s \prec x$ such that $s$ is an injection and $\left\{x_{n}: n \in \omega\right\}=\left\{s_{n}: n \in \omega\right\}$. Let $s_{n}=\left\langle s_{n}^{1}, s_{n}^{2}\right\rangle$. Then, from 6.5 it follows (f).

The sequential space $\left\langle\mathbb{B}, \tau_{s}\right\rangle$ is a Fréchet space (which is equivalent to $u_{\lambda_{A}}=$ $u_{\lambda_{A}}^{2}$ ) iff $\mathbb{B}$ is weakly-distributive and b-cc (see Theorem 5.1.2 or [5, Th. 3.4.]). The similar theorem is valid in the space $\left\langle\mathbb{B}, \mathcal{O}^{\dagger}\right\rangle$.

Theorem 6.2.2 A complete Boolean algebra $\mathbb{B}$ is weakly-distributive and $b$-cc iff $u=u^{2}$ holds in the space $\left\langle\mathbb{B}, \mathcal{O}^{\top}\right\rangle$

Proof.

$1^{\circ}$ Weakly-distributivity an $\mathrm{b}-c c$ implies $u=u^{2}$. Let us suppose that for each decreasing matrix $\left[b_{n k}\right]$ there exists $g \in \omega^{\omega}$ such that $\limsup \left\langle b_{n g(n)}\right\rangle=0$. This is, according to Theorem 4.3 .7 , equivalent to $\mathbb{B}$ is weakly-distributive and b-cc. Let us prove condition (e) from Theorem 6.2.1. Let $\left[a_{n k}\right]$ be a matrix such that the sequence $\left\langle a_{n k}: k \in \omega\right\rangle$ is decreasing for each $n$, and let $\lim \sup _{n} \limsup _{k}\left\langle a_{n k}\right\rangle=$ 0 . For each $n \in \omega$ let limsup $\sup _{k}\left\langle a_{n k}\right\rangle=y_{n}$. So, there holds $y_{n}=\bigwedge_{k \in \omega} a_{n k}$ and that $\limsup \left\langle y_{n}\right\rangle=0$. Let $b_{n k}=a_{n k} \backslash y_{n}$. Since, for each $k \in \omega$ we have 
$y_{n} \leq a_{n k}$, this implies that $a_{n k}=b_{n k} \vee y_{n}$. It is obvious that the sequence $\left\langle b_{n k}: k \in \omega\right\rangle$ is decreasing for each $n \in \omega$ and also that $\bigwedge_{k \in \omega} b_{n, k}=\bigwedge_{k \in \omega}\left(a_{n k}\right)$ $\left.y_{n}\right)=\left(\bigwedge_{k \in \omega} a_{n k}\right) \backslash y_{n}=0$. Therefore, $\left[b_{n k}\right]$ is a decreasing matrix, which implies that there exists a function $f \in \omega^{\omega}$ such that $\lim \sup \left\langle b_{n f(r)}\right\rangle=0$, so $\lim \sup \left\langle a_{n f(n)}\right\rangle=\limsup \left\langle\left(b_{n f(n)} \vee y_{n}\right)\right\rangle=\lim \sup \left\langle b_{n f(n)}\right\rangle \vee \limsup \left\langle y_{n}\right\rangle=$ $0 \vee \lim \sup \left\langle y_{n}\right\rangle=0$. For the sequence $x$ defined by $x_{n}=a_{n f(n)}$ we have $x \in A^{\nu}$ and $\lim \sup x=0$, which completes the first part of the proof,

$2^{\circ} u=u^{2}$ implies weakly-distributivity. Let $u=u^{2}$ and let us suppose that the Boolean algebra $\mathbb{B}$ is not weakly distributive. This is equivalent to $\mathbb{B}$ is not $\omega^{\omega}$ bounding (see Theorem 4.3.6), i.e. in some extension $V_{\mathrm{B}}[G]$ there exists a function $h \in\left(\omega^{\omega}\right)^{V_{\Theta}[G]}$ such that for each function $g \in\left(\omega^{\omega}\right)^{V}$ is not $h \leq^{*} g$. Let $f$ be an increasing function such that $h \leq f$. It is obvious that $f \in V_{\mathbb{B}}[G] \backslash V$ and let $\tau$ be a name such that $f=\tau_{G}$. So, we have

$$
\left[\tau_{G}: \omega \rightarrow \omega \text { is increasing } \wedge \neg \exists g \in\left(\omega^{\omega}\right)^{V} \tau_{G} \leq^{*} g\right]^{V_{B}[G]} .
$$

So, there exists $b \in \mathbb{B}$ such that

$$
b \Vdash \tau: \breve{\omega} \rightarrow \breve{\omega} \text { is increasing } \wedge \neg \exists g \in\left(\left(\omega^{\omega}\right)^{V}\right) \tau \leq^{*} g .
$$

According to Lemma 4.3.5 there exists a nice name $\sigma$ for a subset of $\omega^{2}$ such that

$$
\begin{aligned}
& 1 \Vdash \sigma: \bar{\omega} \rightarrow \bar{\omega} \text { is increasing } \\
& b \Vdash \neg \exists g \in\left(\left(\omega^{\omega}\right)^{V}\right)^{-} \sigma \leq^{*} g .
\end{aligned}
$$

Let $\left\{a_{n}: n \in \omega\right\} \subset \mathbb{B}^{+}$be partition of the unity. Let $\pi$ be a name of a subset of $\omega^{2}$ defined by

$$
\pi=\left\{\left\langle\langle n, k\rangle ;\|\exists l \geq \check{k}\langle n, l\rangle \in \sigma\| \vee a_{n}\right\rangle: n, k \in \omega\right\} .
$$

If $\sigma=\left\{\left\langle\langle n, k\rangle ; a_{n k}\right\rangle: n, k \in \omega\right\}$, then $\|\exists l \geq \bar{k}\langle n, l\rangle \in \sigma\|=\bigvee_{l \geq k} a_{n l}$.

Claim $1, a_{m} \Vdash \pi=\left(\bigcup_{n \in \omega}\{n\} \times\left[0,(\sigma(n))^{-}\right]\right) \cup(\{\tilde{m}\} \times \check{\omega})$.

Proof of Claim 1. Let $a_{m} \in G$, where $G$ is a $\mathbb{B}$-generic filter over $V$, Let us prove that

$$
\pi_{G}=\left(\bigcup_{n \in \omega}\{n\} \times\left[0, \sigma_{G}(n)\right]\right) \cup(\{m\} \times \omega) .
$$

(C) Let $\langle n, k\rangle \in \pi_{G}$. If $n=m$, then $\langle n, k\rangle \in\{m\} \times \omega$. If $n \neq m$, since $\|\exists l \geq \tilde{k}\langle n, l\rangle \in \sigma\| \vee a_{n} \in G$ we have $a_{n} \in G$ or $\|\exists l \geq \tilde{k}\langle n, l\rangle \in \sigma\| \in G$. The first case is impossible, so there exists $l \geq k$ such that $\langle n, l\rangle \in \sigma_{G}$, which implies that $k \in\left[0, \sigma_{G}(n)\right]$, and finally that $\langle n, k\rangle \in\{n\} \times\left[0, \sigma_{G}(n)\right]$. 
(D) Since $a_{m} \in G$, we have $\left\|\exists l \geq \hat{k}\langle m, l\rangle^{-} \in \sigma\right\| \vee a_{m} \in G$, which implies that for each $k \in \omega,\langle m, k\rangle \in \pi_{G}$, i.e. $\{m\} \times \omega \subset \pi_{G}$. Let $n \in \omega$ and $k \leq \sigma_{G}(n)$. Then, there exists $l \geq k$ such that $\langle n, l\rangle \in \sigma_{G}$. Therefore, $\|\exists l \geq \tilde{k}\langle n, l\rangle \in \sigma\| \in$ $G$, which implies that $\|\exists l \geq \tilde{k}\langle n, l\rangle \in \sigma\| \vee a_{n} \in G$, i.e. $\langle n, k\rangle \in \pi_{G}$, which completes the proof of Claim 1 .

Let us prove that $\pi$ fulfils conditions from Theorem 6.2.1 (f). For each $n, k \in \omega$ we have $\|\langle n, k\rangle \in \pi\| \geq a_{n}>0$. Let $G$ be a $\mathbb{B}$-generic filter over $V$. Then, there exists only one $m \in \omega$ such that $a_{m} \in G$. By (6.8), for $n>m$ we have that $\pi_{G} \cap(\{n\} \times \omega)=\{n\} \times[0, \sigma(n)]$ is finite.

From equivalence of (a) and (f) in Theorem 6.2.1 it follows that there exists $A \in\left[\omega^{2}\right]^{\omega}$ such that

$$
1 \Vdash\|\pi \cap \tilde{A}\|<\dot{\omega} .
$$

Claim 2. For each $n \in \omega$ the set $A \cap(\{n\} \times \omega)$ is finite.

Proof of Claim 2. Let $n \in \omega$ and let $a_{n} \in G$, where $G$ is a $\mathbb{B}$-generic filter over $V$. By (6.9) we have that $\left|\pi_{G} \cap A\right|<\omega$. According to Claim 1, $a_{n} \Vdash(\{n\} \times \omega) \subset \pi_{\text {, }}$ which implies that $\{n\} \times \omega \subset \pi_{G}$. Therefore $(\{n\} \times \omega) \cap A \subset \pi_{G} \cap A$, which implies that $|(\{n\} \times \omega) \cap A|<\omega$, which completes the proof of Claim 2 .

Claim 2 implies that the set

$$
S=\{n \in \omega: A \cap(\{n\} \times \omega) \neq \emptyset\},
$$

the first projection of the set $A$, is infinite. Let $\varphi: S \rightarrow \omega$ be defined by

$$
\varphi(n)=\min \{k \in \omega:\langle n, k\rangle \in A\} .
$$

Clearly, $\varphi \subset A$. Let $b \in G$, where $G$ is a $\mathbb{B}$-generic filter over $V$. Since (6.9) implies that $\left|\pi_{G} \cap A\right|<\omega$, we have that

$$
\left|\pi_{G} \cap \varphi\right|<\omega .
$$

Therefore, there exists $r \in \omega$ such that, for each $n \in S \backslash r\langle n, \varphi(n)\rangle \notin \pi_{G} \cap$ $(\{n\} \times \omega)$.

Since $\left\{a_{n}: n \in \omega\right\}$ is the partition of the unity, there exists a unique $m_{n} \in \omega$ such that $a_{m} \in G$. Equation (6.8) implies that, for each $n>m, \pi_{G} \cap(\{n\} \times$ $\omega)=\{n\} \times\left[0, \sigma_{G}(n)\right]$. Hence, for each $n \in S \backslash \max \{r, m+1\}$, we have $\langle n, \varphi(n)\rangle \notin\{n\} \times\left[0, \sigma_{G}(n)\right]$, i.e. $\sigma_{G}(n)<\varphi(n)$, So, for $n_{0}=\max \{r, m+1\}$ we have

$$
\forall n \in S \backslash n_{0} \sigma_{G}(n)<\varphi(n) .
$$

Let $g: \omega \rightarrow \omega$ be defined with

$$
g(n)=\varphi(\min S \cap[n, \omega)), n \in \omega,
$$


The fact that $\varphi \in V$ implies that $g \in V$.

Claim 3. $\forall n \geq n_{0} \sigma_{G}(n)<g(n)$.

Proof of Claim 3, Let $n \geq n_{0}$. For $n_{1}=\min (S \cap[n, \omega))$ we have $S \ni n_{1} \geq n \geq$ $n_{0}$, so $n_{1} \in S \backslash n_{0}$. Equations (6.10) and (6.6) imply

$$
g(n)=\varphi\left(n_{1}\right)=\sigma_{G}\left(n_{1}\right) \geq \sigma_{G}(n)
$$

which proves Claim 3.

So, for the function $g$ there holds $\sigma_{G} \leq^{\star} g$. Since $b \in G$, it contradicts with (6.7).

$3^{\circ} u=u^{2}$ implies $\mathfrak{b}-c c$. Let us suppose that the Boolean algebra $\mathbb{B}$ is not $b$-cc. Then there exists a partition of the unity $\left\{a_{\alpha}: \alpha<b\right\} \subset \mathbb{B}^{+}$. Let $\left\{f_{\alpha}: \alpha<b\right\} \subset$ $\omega^{\omega}$ be an unbounded family of increasing functions. Then, for the name

$$
\tau=\left\{\left\langle\langle n, k\rangle, \bigvee\left\{a_{\alpha}: \alpha<b \wedge\langle n, l\rangle \in f_{\alpha}\right\}\right\rangle: n, k \in \omega\right\}
$$

we have $a_{\alpha}$ It $\tau=f_{\alpha}$. We will create a name $\pi$ for a subset of $\omega^{2}$ with the elements under the graphic of the function $\tau$ which contains a set of a form $\{n\} \times \omega$. Let $\left\{c_{n}: n \in \omega\right\}$ be a partition of the unity and let

$$
\pi=\left\{\left\langle\langle n, k\rangle, V_{l \geq k} \bigvee\left\{a_{\alpha \alpha}: \alpha<\mathfrak{b} \wedge\langle n, l\rangle \in f_{\alpha}\right\} \vee c_{n}\right\rangle: n, k \in \omega\right\} .
$$

Claim 1. $a_{\alpha_{0}} \wedge c_{m i} \Vdash \pi=\bigcup_{n \in \dot{\omega}}\left(\{n\} \times\left[0, f_{\alpha_{0}}(\tilde{n})\right]\right) \cup(\{\check{m}\} \times \omega)$.

Proof of Claim 1, Let $a_{\alpha_{0}} \wedge c_{m} \in G$, where $G$ is a generic filter over $\mathbb{B}$. Let us prove that

$$
\pi_{G}=\bigcup_{n \in \omega}\left(\{n\} \times\left[0, f_{\alpha_{0}}(n)\right]\right) \cup(\{m\} \times \omega) .
$$

(C) Let $\langle n, k\rangle \in \pi_{G}$. Then $\|\langle n, k\rangle \in \pi\| \in G$, so we have two possibilities.

(i) $c_{n} \in G$. Since $G \ni a_{\alpha_{0}} \wedge c_{m} \leq c_{m}$, we have $c_{m} \in G$, which implies that $m=n$. Therefore, $\langle n, k\rangle=\langle m, k\rangle \in\{m\} \times \omega$.

(ii) $c_{n} \notin G$. Then $\bigvee_{l>k} \bigvee\left\{a_{\alpha}: \alpha<b \wedge\langle n, l\rangle \in f_{\alpha}\right\} \in G$. The fact that $a_{\alpha_{0}} \in G$ implies that $\bigvee_{l \geq k} \bigvee\left\{a_{\alpha} \wedge a_{\alpha_{0}}: \alpha<\mathrm{b} \wedge\langle n, l\rangle \in f_{\alpha}\right\} \in G$. For $\alpha \neq \alpha_{0}$ we have $a_{\alpha} \wedge a_{\alpha_{0}}=0$. Therefore,

$$
\left\{a_{\alpha} \wedge a_{\alpha_{0}}: \alpha<b \wedge\langle n, l\rangle \in f_{\alpha}\right\}= \begin{cases}\{0\} & \langle n, l\rangle \notin f_{\alpha_{0}} \\ \left\{a_{\alpha_{0}}\right\} & \langle n, l\rangle \in f_{\alpha_{0}}\end{cases}
$$

There exists $l \geq k$ such that $\langle n, l\rangle \in f_{\alpha_{0}}$, since, otherwise would be $0 \in G$. So, $f_{\alpha_{0}}(n)=l \geq k$ implies that $\langle n, k\rangle \in\{n\} \times\left[0, f_{\alpha_{0}}(n)\right]$.

(D) Let $\langle n, k\rangle$ be an element of the set on the right-hand side of equation (6.11). If $n=m$ then $c_{m} \in G$ implies that $\|\langle m, k\rangle \in \pi\| \in G$, leading to 
$\langle n, k\rangle=\langle m, k\rangle \in \pi_{G}$. If $n \neq m$, then $k \leq f_{\alpha_{0}}(n)=l_{0}$ which implies that $a_{\alpha_{0}} \in\left\{a_{\alpha}: \alpha<\mathfrak{b} \wedge\left\langle n, l_{0}\right\rangle \in f_{\alpha}\right\}$. So,

$$
\begin{aligned}
a_{\alpha_{0}} & \leq \bigvee\left\{a_{\alpha}: \alpha<\mathrm{b} \wedge\left\langle n, l_{0}\right\rangle \in f_{\alpha}\right\} \\
& \leq \bigvee_{l \geq k} \bigvee\left\{a_{\alpha}: \alpha<\mathrm{b} \wedge\left\langle n_{2} l_{0}\right\rangle \in f_{\alpha}\right\} \\
& \leq\|\langle n, k\rangle \in \pi\| .
\end{aligned}
$$

This implies that $\|\langle n, k\rangle \in \pi\| \in G$, i.e. $\langle n, k\rangle \in \pi_{G}$, completing the proof of Claim 1 .

Let us prove that $\pi$ fulfils conditions from Theorem 6.2.1 (f).

For $n, k \in \omega$ we have $\|\langle n, k\rangle \in \pi\| \geq c_{n}>0$. Let $G$ be a $\mathbb{B}$-generic filter over $V$. Then there exist unique $\alpha_{0}<\mathfrak{b}$ and $m \in \omega$ such that $a_{\alpha_{0}}, c_{m} \in G$, which implies that $a_{\alpha_{0}} \wedge c_{m} \in G$. Claim 1 implies that for each $n>m$ we have $|\pi \cap(\{n\} \times \omega)|<\omega$.

Equivalence of (a) and ( $\mathrm{f}$ ) in Theorem 6.2.1 implies that there exists an infinite set $A \subset \omega^{2}$ such that

$$
1 \Vdash|\pi \cap \breve{A}|<\breve{\omega} .
$$

Claim 2. For each $m \in \omega,|A \cap(\{m\} \times \omega)|<\omega$.

Proof of Claim 2. For $m \in \omega$ there exists $\alpha<$ b such that $a_{\alpha} \wedge c_{m}>0$. Let $G$ be a generic filter over $\mathbb{B}$ containing $a_{\alpha} \wedge c_{m}$. Equation (6.12) implies that $\left|\pi_{G} \cap A\right|<$ $\omega$. This and Claim 1 implies equation (6.12), which gives that $\{m\} \times \omega \subset \pi_{G}$. Therefore, $A \cap(\{m\} \times \omega)$, as a subset of the finite set $A \cap \pi_{G}$, is finite. This completes the proof of Claim 2.

Let

$$
S=\{n \in \omega: A \cap(\{n\} \times \omega) \neq \emptyset\},
$$

and $\varphi: S \rightarrow \omega$ be defined with

$$
\varphi(n)=\min \{k \in \omega:\langle n, k\rangle \in A\} .
$$

$\varphi \subset A$ and (6.12) implies

$$
1 \Vdash|\pi \cap \tilde{\varphi}|<\tilde{\omega} .
$$

Let $\alpha<\boldsymbol{b}$ and let $G$ be a generic filter containing $a_{\alpha}$. Then $\left|\pi_{G} \cap \varphi\right|<\omega$. So, there exists $r \in \omega$ such that

$$
\forall n \in S \backslash r\langle n, \varphi(n)\rangle \notin \pi_{G} \cap(\{n\} \times \omega) .
$$

There exists $m \in \omega$ such that $c_{m} \in G$, which implies that $a_{\alpha} \wedge c_{m} \in G$. Claim I. implies that

$$
\forall n>m \pi_{G} \cap(\{n\} \times \omega)=\{n\} \times\left[0, f_{\alpha}(n)\right] .
$$


Let $n_{0}=\max \{r, m+1\}$. Then, from equations (6.14) and (6.15) we obtain that

$$
\forall n \in S \backslash n_{0}\langle n, \varphi(n)\rangle \notin\{n\} \times\left[0, f_{\alpha}(n)\right],
$$

i.e. $f_{\alpha}(n)<\varphi(n)$. So

$$
\forall n \in S \backslash n_{0} f_{\alpha}(n)<\varphi(n) .
$$

Let $g: \omega \rightarrow \omega$ be defined with

$$
g(n)=\varphi(\min S \cap[n, \omega)), n \in \omega .
$$

Since $f_{\alpha}$ is an increasing function, (6.16) implies that

$$
\forall n \geq n_{0} f_{\alpha}(n)<g(n)
$$

which implies that $f_{\alpha} \leq \leq^{*}$. The fact that this is true for each $\alpha<\mathrm{b}$ contradicts unboundness of the family $\left\{f_{a}: \alpha<\boldsymbol{b}\right\}$.

The examples of Boolean algebras related to $\mathfrak{b}$ - $\mathrm{cc}$ and weak-distributivity are given in Remark 4.3 .8 on page 46.

\subsection{A posteriori limit in $\left\langle\mathbb{B}, \mathcal{O}^{\top}\right\rangle$}

Using the a priori limit operator $\lambda^{\top}$ defined on a c.B.a. $\mathbb{B}$ we have obtained the topological space $\left\langle\mathbb{B}, \mathcal{O}^{\top}\right\rangle$ with the a posteriori limit $\lim _{\mathcal{O}}$, which will be in this section briefly denoted by $\mathrm{lim}$. In this section we will investigate it on an arbitrary complete Boolean algebra.

Theorem 6.3.1 In the space $\left\langle\mathbb{B}, \mathcal{O}^{\top}\right\rangle$ we have

(a) $\lim x=\bigcap\left\{F \in \mathcal{F}^{\top}: x_{n} \in F\right.$ for infinitely many $\left.n \in \omega\right\}$

$$
\begin{aligned}
& =\bigcap_{f \in \omega T \omega} \mathrm{cl}_{\omega_{1}}\left(\left\{x_{f(n)}: n \in \omega\right\}\right) \\
& =\bigcap_{A \in[\omega] \omega} \operatorname{cl}_{\omega_{1}}\left(\left\{x_{n}: n \in A\right\}\right)
\end{aligned}
$$

(b) $(\lim \sup x) \uparrow \subset \lim x \subset \mathrm{cl}_{\omega_{1}}\left(\left\{x_{n}: n \in \omega\right\}\right)$

Proof. It follows directly from Fact 2.2.1 (a) and (b).

Corollary 6,3.2 The a posteriori limit of a sequence is an upward closed set, i.e. $\lim x=\lim x \uparrow$. 
Proof. According to Fact 2.2.1 (b), the a posteriori limit of a sequence is always a closed set, and, by Theorem 6.1.2, it is upward closed.

Example 6.3.3 For a constant sequence $\langle b: n \in \omega\rangle$ we have $\lim \langle b\rangle=b \uparrow$. If $x=\langle a, b, a, b, \ldots\rangle$, then $\lim x=(a \vee b) \uparrow$. This will follow from the following, more general, theorem.

Theorem 6.3.4 Let $x=\left\langle x_{n}: n \in \omega\right\rangle$ be a sequence with finite range, i.e. the set $\left\{x_{n}: n \in \omega\right\}$ is finite and $C=\left\{b: x_{n}=b\right.$ for infinitely many $\left.n \in \omega\right\}$. Then

$$
\lim \left\langle x_{n}\right\rangle=(\bigvee C) \uparrow
$$

Proof. Let $a=\bigvee C$. For each $c \in C$, the set $c \uparrow$ is closed and contains infinitely many members of the sequence $x$. Since $\bigcap_{c \in C}(c \uparrow)=\bigvee C \uparrow$ is a closed set and, according to Theorem 6.3.1, there bolds $\lim \left\langle x_{n}\right\rangle \subset \bigvee C \uparrow=a \uparrow$. Let us suppose that there exists $d \in \lim \left\langle x_{n}\right\rangle \backslash(a \uparrow)$. Then we have that $a \notin d$, which implies that $a \wedge d^{\prime} \& d$. But $\left(a \wedge d^{\prime}\right) \uparrow$ also contains infinitely many members of the sequence, but does not contain $d$. Therefore, by Theorem 6.3.1, we have that $d \notin \lim \left\langle x_{n}\right\rangle$.

In the forthcoming theorems we will investigate connections between the a posteriori limit operator and limsup of a sequence.

Theorem 6.3.5 If $\lim \sup x=0$ then $0 \in \lim x$, and therefore $\lim x=\mathbb{B}$.

Proof. By Theorem 6.3.1 (b), $\mathbb{B}=0 \uparrow \subset(\lim \sup x) \uparrow \subset \lim x \subset \mathbb{B}$.

Lemma 6.3.6 In the Boolean algebra $P(\omega)$ there holds

$$
0 \in \lim x \Leftrightarrow \lim \sup x=0 .
$$

Proof. By the previous theorem we have that $\lim \sup x=0$ implies $0 \in \lim x$.

Let us prove the inverse implication. Let us suppose that there exists a sequence $x=\left\langle x_{n}: n \in \omega\right\rangle$ in $P(\omega)$ such that $0 \in \lim x$ and $\lim \sup x=c>0$. Let $c_{0} \in c$. The set $\mathbb{B} \backslash\left(\left\{c_{0}\right\} \uparrow\right)$ is an open set containing 0 , so there exists $n_{0}$, such that $x_{n} \in \mathbb{B} \backslash\left(\left\{c_{0}\right\} \uparrow\right)$ for each $n \geq n_{0}$. So, $x_{n} \notin\left\{c_{0}\right\} \uparrow$, i.e. $c_{0} \notin x_{n}$, for each $n \geq n_{0}$, which contradicts the fact that $c_{0} \in \lim \sup x$.

Lemma 6.3.7 Let $\mathbb{B}$ be a c.B.a. such that $\lim \neq \lambda^{\dagger}$. Then there exists a sequence $x$ such that $0 \in \lim x \backslash \lambda^{\top}(x)$. 
Proof. Let $x$ be a sequence in $\mathbb{B}$ such that there exists $b \in \lim x \backslash \lambda^{\top}(x)$. Then lim $\sup x \not b$. This implies that $c=\lim \sup x \wedge b^{\prime}>0$. From this it follows that $c \not b$, i,e. $b \in \mathbb{B} \backslash(c \uparrow)$. Hence, $\mathbb{B} \backslash(c \uparrow)$ is an open neighborhood of $b$, and therefore

$$
\exists n_{0} \in \omega \forall n \geq n_{0} x_{n} \in \mathbb{B} \backslash(c \uparrow),
$$

i.e.

$$
\exists n_{0} \in \omega \forall n \geq n_{0} x_{n} \not \subset c .
$$

Since $c \leq \limsup x=\bigwedge_{k \in \omega} \bigvee_{n \geq k} x_{n}$, we have $c=\bigwedge_{k \in \omega} \bigvee_{n \geq k} x_{n} \wedge c$.

Let us define $y_{n}=x_{n} \wedge c, n \in \omega$. Then $c=\lim \sup \left\langle y_{n}\right\rangle$, so, $0 \notin \lambda^{\dagger}\left(\left\langle y_{n}\right\rangle\right)$. Let us prove that $0 \in \lim \left(\left\langle y_{n}\right\rangle\right)$. From $b \in \lim x$ and continuity of the function $f_{c}: \mathbb{B} \rightarrow \mathbb{B}, f_{c}(x)=x \wedge c$ (see Lemma 6.1.5 and Fact 2.2.6 (a)) it follows that $0=b \wedge c \in \lim \left\langle x_{n} \wedge c\right\rangle$.

So, we have seen that if a priori and a posteriori operator do not coincide, then the family of sequence which converges to 0 is different for these two operators. In the sequel we will give a sufficient condition for a sequence to converge to a point, which is similar to condition given in Theorem 5.1 .1 for the sequential topology $\tau_{s}$. For this, we need the following lemma.

Lemma 6.3.8 Let $\left\langle x_{n}\right\rangle$ and $\left\langle z_{n}\right\rangle$ be two sequences in $\mathbb{B}$ such that $x_{n} \leq z_{n}, n \in \omega$. Then $\lim z_{n} \subset \lim x_{n}$.

Proof. It follows directly from Theorem 6.3.1 (a) and the fact that each closed set is upward closed.

Theorem 6.3.9 Let $\mathbb{B}$ be a c.B.a. Then, if for a sequence $x$ and an element $a$ we have

$$
\forall y \prec x \exists z \prec y \limsup z \leq a,
$$

then $a \in \lim x$.

Proof. Let us suppose that there exists a sequence $x$ and an element $a$ such that for each $y \prec x$ there exists $z \prec y$ such that $\lim \sup z \leq a$ and $a \notin \lim x$. Then there exists a closed set $F$ such that $a \notin F$ and for each $n_{0}$ there exists $m \geq n_{0}$ such that $x_{m} \in F$. Therefore there exists $y^{0} \prec x$ such that $y^{0} \in F^{w}$ and $z^{0} \prec y^{0}$ such that limsup $z^{0} \leq a$. Obviously $z^{0} \in F^{\omega}$ and there holds (limsup $\left.z^{0}\right) \uparrow=\lambda^{\top}\left(z_{0}\right) \subset$ $\lim _{\mathcal{O} ;} z^{0} \subset F$. This implies that $a \in F$. A contradiction.

But, in the class of lim sup-stable algebras (algebras with condition $(\hbar)$ ), statement (6.17) is a characterization of the a posteriori limit. 
Theorem 6.3.10 Let $\mathbb{B}$ be a c.B.a. satisfying $(\hbar)$. Then, for each sequence $x$ we have

$$
a \in \lim x \Leftrightarrow \forall y \prec x \exists z \prec y \limsup z \leq a .
$$

Proof. $(\Leftrightarrow)$ proven in Theorem 6.3.9.

$(\Rightarrow)$ Let $x$ be a sequence in $\mathbb{B}$ such that $a \in \lim _{\mathcal{O}} x$ and let us suppose that there exists a sequence $y \prec x$ such that for all $z \prec y$ we have lim $\sup z \not a$. Since $\mathbb{B}$ satisfies $(\hbar)$, there exists a sequence $z^{0}=\left\langle z_{n}^{0}: n \in \omega\right\rangle \prec y$ such that

$$
\forall z^{1} \prec z^{0} \lim \sup z^{1}=\lim \sup z^{0} .
$$

Without lost of generality we can suppose that $z_{n}^{0} \mathbb{E} a, n \in \omega$. Let us prove that

$$
c l_{\omega_{1}}\left(\left\{z_{n}^{0}: n \in \omega\right\}\right)=\bigcup_{n \in \omega}\left(z_{n}^{0} \uparrow\right) \cup\left(\limsup z^{0}\right) \uparrow .
$$

(つ) We have that $\lambda^{\dagger}\left(\left\langle z_{n}^{0}: n \in \omega\right\rangle\right)=\left(\limsup z^{0}\right) \uparrow$ and $\lambda^{\top}\left(\left\langle z_{n}^{0}\right\rangle\right)=z_{n}^{0} \uparrow$ for each $n$, where $\left\langle z_{n}^{0}\right\rangle$ is a constant sequence. Therefore

$$
\bigcup_{n \in \omega}\left(z_{n}^{0} \uparrow\right) \cup\left(\limsup z^{0}\right) \uparrow \subset u_{\lambda \Gamma}\left(\left\{z_{n}^{0}: n \in \omega\right\}\right) \subset c l_{\omega_{1}}\left(\left\{z_{n}^{0}: n \in \omega\right\}\right) .
$$

Claim 1 .

(C) Let us firstly prove

$$
u_{\lambda \uparrow}\left(\left\{z_{n}^{0}: n \in \omega\right\}\right)=\bigcup_{n \in \omega}\left(z_{n}^{0} \uparrow\right) \cup\left(\limsup z^{0}\right) \uparrow .
$$

Proof of Claim 1. Let $v$ be a sequence in $\left\{z_{n}^{0}: n \in \omega\right\}$. If $v$ has a constant subsequence, namely $\left\langle z_{k}^{0}\right\rangle$, then $z_{k}^{0} \leq \lim \sup v$, which implies $\lambda^{\top}(v) \subset z_{k}^{0} \uparrow$. If $v$ does not have a constant subsequence, then it has a subsequence $u$ which is also a subsequence of $z^{0}$. Therefore, by (6.18), we have

$$
\lambda^{\top}(v)=(\lim \sup v) \uparrow \subset(\lim \sup u) \uparrow=\left(\lim \sup z^{0}\right) \uparrow .
$$

This completes the proof of Claim 1 .

Claim 2.

$$
u_{\lambda \tau}\left(\left\{z_{n}^{0}: n \in \omega\right\}\right)=u_{\lambda \tau}\left(u_{\lambda T}\left(\left\{z_{n}^{0}: n \in \omega\right\}\right)\right) .
$$

Proof of Claim 2. Let $t=\left\langle t_{k}: k \in \omega\right\rangle$ be a sequence in $u_{\lambda \uparrow}\left(\left\{z_{n}^{0}: n \in \omega\right\}\right)$. Then, by (6.19), for each $k$ we have $\lim \sup z^{0} \leq t_{k}$ or there exists $n_{k} \in \omega$ such that $z_{n_{k}}^{0} \leq t_{k}$. If $t$ has a subsequence $\left\langle t_{k_{l}}: l \in \omega\right\rangle$ such that for each $l \in \omega$ we have $\limsup z^{0} \leq t_{k_{l}}$, then $\lim \sup z_{0} \leq \lim \sup \left\langle t_{k_{l}}: l \in \omega\right\rangle \leq \limsup t$. If not, then there exists a subsequence $\left\langle t_{k_{m}}: m \in \omega\right\rangle$ such that $z_{n_{k_{m}}}^{0} \leq t_{k_{m}}$. Then

$$
\limsup z^{0}=\lim \sup \left\langle z_{n_{k_{m}}}^{0} ; m \in \omega\right\rangle \leq \limsup \left\langle t_{k_{m}}: m \in \omega\right\rangle \leq \limsup t .
$$

This proves Claim 2. 
The set $U=\mathbb{B} \backslash c_{\omega_{1}}\left(\left\{z_{n}^{0}: n \in \omega\right\}\right)$ is an open set containing the point $a$ such that $n_{0}$ there exists $n_{1} \geq n_{0}$ such that $z_{n_{1}}^{0} \notin U$. So, $a \notin \lim z^{0}$, and since each a posteriori limit operator satisfy (L2), we have $a \notin \lim x$. A contradiction.

As a consequence of the previous theorem we have obtain the equality between Fréchet spaces and spaces satisfying condition $u=u^{2}$ in the class of Boolean algebras satisfying $(\hbar)$.

Theorem 6.3.11 Let $\mathbb{B}$ be a c.B.a. satisfying $(\hbar)$. Then $\left\langle\mathbb{B}, \mathcal{O}^{\top}\right\rangle$ is a Fréchet space iff $u=u^{2}$.

Proof. By Theorem 2.3.18, if $u=u^{2}$, then the space is Fréchet. Let us prove the inverse implication. Let $\left\langle\mathbb{B}, \mathcal{O}^{\dagger}\right\rangle$ be a Fréchet space, and let $A \subset \mathbb{B}$. Let us prove that $u(A)=c l_{\omega_{1}}(A)$. Let us suppose that there exists $a \in c l_{\omega_{1}}(A) \backslash u(A)$. Therefore, there exist a sequence $x$ in $A$ such that $a \in \lim x$, and for each sequence $y \in A^{\omega} \limsup y \not \leq a$, which contradicts Theorem 6.3.10.

\subsection{A posteriori limit in case when $u=u^{2}$}

We have seen that a c.B.a. is weakly distributive and b-cc iff there holds $u=u^{2}$ (see Theorem 6.2.2). The properties of the topological space $\left\langle\mathbb{B}, \mathcal{O}^{\dagger}\right\rangle$, where $\mathbb{B}$ is a $\mathfrak{b}-c c$ and weakly distributive complete Boolean algebra will be investigated.

In the majority of the theorems presented in this section we will presume that a Boolean algebra is weakly distributive and b-cc. Therefore, these properties of a complete Boolean algebra will be briefly denoted by $\left(u=u^{2}\right)$ in the beginning of statements.

Lemma 6.4.1 $\left(u=u^{2}\right)$ If $b \in \lim x$, then there exists $y \prec x$ such that $\lim \sup y \leq$ $b$.

Proof, Let $b \in \lim x$. If there exists a subsequence $y \prec x$ such that $y_{n} \leq b, n \in \omega$, then, obviously, there holds limsup $y \leq b$.

In opposite, there exists $m \in \omega$ such that $x_{n} \nsucceq b$ for all $n \geq m$. According to Fact 2.2.1 (d), we have $\lim x=\lim \left\langle x_{n}: n \geq m\right\rangle$. Therefore,

$$
b \in \lim \left\langle x_{n}: n \geq m\right\rangle \subset \overline{\left\{x_{n}: n \geq m\right\}}=u\left(\left\{x_{n}: n \geq m\right\}\right) .
$$

This implies that there exists a sequence $z \in\left\{x_{n} ; n \geq m\right\}^{\omega}$ such that $\lim \sup z \leq$ $b$. The sequence $z$ does not have a constant subsequence $\left\langle x_{n_{0}}\right\rangle$, since $x_{n_{0}}=$ $\limsup \left\langle x_{n_{0}}\right\rangle \leq \limsup z \leq b$, which would contradict the fact that $x_{n_{0}} \leq b$. So, $z$ is finite-to-one mapping. One can easily define a subsequence $z^{0} \prec z$ such 
that $z^{0}=\left\langle z_{n}^{0}: n \in \omega\right\rangle$ is one-to-one mapping. So, $\left\{z_{n}^{0} ; n \in \omega\right\} \subset\left\{x_{n} \div n \geq m\right\}$. Therefore, there exists an injection $f: \omega \rightarrow \omega$ such that $z_{n}^{0}=x_{f(n)}$. Obviously

$$
\lim \sup x \circ f=\lim \sup z^{0} \leq \lim \sup z \leq b .
$$

Let $g$ be a bijection which maps $\omega$ onto $\omega$ such that $f \circ g$ is a strictly increasing mapping. So, $x \circ f \circ g \prec x$ and, by Lemma 3.3.6, $b \geq \lim \sup x$ a $f=\lim \sup x \circ$ $f \circ g$, which competes the proof.

Example 6.4.2 The inverse implication must not be true. The limit of the sequence $\langle a, b, a, b, \ldots\rangle)$ is $(a \vee b) \uparrow$, for incomparable $a$ and $b$, but for the subsequence $\langle a\rangle$ we have $a=\lim \sup \langle a\rangle$ and $a \notin \lim \langle a, b, a, b, \ldots\rangle$

Theorem 6.4.3 $\left(u=u^{2}\right)$ Let $x \in \mathbb{B}^{\omega}$ and $b \in \mathbb{B}$. Then the following conditions are equivalent:

(a) $b \in \lim x ;$

(b) $\forall f \in \omega^{\top \omega} \exists y \in\left\{x_{f(n)}: n \in \omega\right\}^{\omega} \limsup y \leq b$;

(c) $\forall A \in[\omega]^{\omega} \exists y \in\left\{x_{n} ; n \in A\right\}^{\omega}$ limsup $y \leq b$;

(d) $\forall A \in[\omega]^{\omega} \exists g: \omega \rightarrow A \| \check{g}^{-1}\left[\tau_{x}\right]$ is infinite $\| \leq b$;

(e) $\forall f \in \omega^{\uparrow \omega} \exists g \in \omega^{T \omega} \limsup x \circ f \circ g \leq b$;

(f) $\forall y \prec x \exists z \prec y \lim \sup z \leq b$;

(g) $\forall y \prec x \exists z \prec y b \in \lambda^{\dagger}(z)$;

(h) $\forall A \in[\omega]^{\omega} \exists B \in[A]^{\omega} \| \tau_{x} \cap \check{B}$ is infinite $\| \leq b$;

(i) $\forall A \in[\omega]^{\omega} \exists B \in[A]^{\omega} b^{\prime} \Vdash \tau_{x} \cap \bar{B}$ is finite;

(j) There exists a tall ideal $\mathcal{J}$ on $\omega$ such that $b^{\prime} \Vdash \forall B \in \mathcal{J} \tau_{x} \cap B$ is finite;

(k) $\mathcal{J}_{x}=\left\{B \subset \omega: b^{\prime} \Vdash \tau_{x} \cap \tilde{B}\right.$ is finite $\} \in \operatorname{Tall}(\omega) \cup\{P(\omega)\}$;

Proof. (a) $\Rightarrow$ (b) Let $f \in \omega^{\dagger \omega}$. Property (L2) implies that $b \in \lim x \circ f$. Since $\lim x \circ f \subset \operatorname{cl}_{\omega_{1}}\left(\left\{x_{f(n)}: n \in \omega\right\}\right)=u\left(\left\{x_{f(n)}: n \in \omega\right\}\right)$, there exists $y \in\left\{x_{f(n)}\right.$ : $n \in \omega\}^{\omega}$ such that limsup $y \leq b$.

(b) $\Leftrightarrow$ (c) It is obvious.

(c) $\Rightarrow$ (a) Let us suppose that $b \notin \lim x$. Then there exists an open set $O$ containing $b$ such that the set $A=\left\{n \in \omega: x_{n} \notin O\right\}$ is infinite. Therefore there exists a sequence $y$ in $\left\{x_{n}: n \in A\right\}$ such that limsup $y \leq b$. So, $b \in \operatorname{cl}_{\omega_{1}}\left(\left\{x_{n}\right.\right.$ : $n \in A\}) \subset \mathbb{B} \backslash O$, contradicting the fact that $b \in O$.

(c) $\Rightarrow$ (d) Let $A \subset[\omega]^{\omega}$. (c) implies that there exists a sequence $y=\left\langle y_{k}: k \in\right.$ $\omega) \in\left\{x_{n}: n \in A\right\}^{\omega}$ such that $\limsup y \leq b$. For each $k \in \omega$ there exists $n \in A$ such that $y_{k}=x_{n}$, and let $g: \omega \rightarrow A$ be defined by $g(k)=\min \left\{n \in A: y_{k}=\right.$ $\left.x_{n}\right\}$. So, $b \geq \lim \sup x \circ g=\bigwedge_{l \in \omega} \bigvee_{k \geq l}\left\|(g(k))^{*} \in \tau_{x}\right\|=\| \forall l \in \dot{\omega} \exists k \geq l k \in$ $\check{g}^{-1}\left[\tau_{x}\right]\|=\| \dot{g}^{-1}\left[\tau_{x}\right]$ is infinite $\|$. 
(d) $\Rightarrow$ (c) Let $A \subset[\omega]^{\omega}$. (d) implies that there exists a function $g: \omega \rightarrow A$ such that $\| \vec{g}^{-1}\left[\tau_{x}\right]$ is infinite $\| \leq b$. Then we have $\limsup \left\langle x_{g(k)}\right\rangle \leq b$ and for $y=\left\langle y_{k}\right\rangle=\left\langle x_{g(k)}\right\rangle$ we have $y \in\left\{x_{n}: n \in A\right\}^{\omega}$ and $\limsup y \leq b$.

(e) $\Rightarrow$ (b) Let $f \in \omega^{\dagger \omega}$. By (e), there exists $g \in \omega^{i \omega}$ such that $\limsup \left\langle x_{f(g(k))}\right\rangle \leq b$. For the sequence $y=\left\langle y_{k}\right\rangle=\left\langle x_{f(g(k))}\right\rangle$ there holds $y \in\left\{x_{f(n)}: n \in \omega\right\}^{\omega}$ and limsup $y \leq b$.

(a) $\Rightarrow$ (e) Let $\in \lim x$. Then for each $f \in \omega^{\top \omega}, b \in \lim x \circ f$, and from Lemma 6,4.1 it follows that there exists $g \in \omega^{\dagger \omega}$ such that $\lim \sup x \circ f \circ g \leq b$.

(e) $\Leftrightarrow(\mathrm{f}) \Leftrightarrow$ (g) It is obvious.

(e) $\Rightarrow$ (h) Let $A \in[\omega]^{\omega}$ and let $f: \omega \rightarrow A$ be the increasing bijection. By (e), there exists $g \in \omega^{\dagger \omega}$ such that limsup $x_{f(g(n))} \leq b$. Let $B=f[g[\omega]]$.

Clearly, $B \subset f[\omega]=A$ and, since $f$ and $g$ are injections, $B$ is infinite. So, we have

$$
\begin{aligned}
b & \geq \limsup x_{f(g(n))} \\
& =\bigwedge_{k \in \omega} V_{n \geq k}\left\|((f \circ g)(n)) \in \tau_{x}\right\| \\
& =\left\|\forall k \in \tilde{\omega} \exists n \geq k n \in\left((f \circ g)^{-1}\right)\left[\tau_{x}\right]\right\| \\
& =\|\left((f \circ g)^{-1}\right)\left[\tau_{x}\right] \text { is infinite } \| \\
& =\| \tau_{x} \cap((f \circ g)[\omega]) \text { is infinite } \quad \text { (since } f \circ g \text { is an injection) } \\
& =\| \tau_{x} \cap \tilde{B} \text { is infinite } \| .
\end{aligned}
$$

(h) $\Rightarrow$ (e) Let $f \in \omega^{\uparrow \omega}$ and let $A=f[\omega]$. Then there exists $B \in[A]^{\omega}$ such that $\| \tau_{x} \cap \tilde{B}$ is infinite $\| \leq b$. Since $f^{-1}[B] \in[\omega]^{\omega}$, there exists an increasing bijection $g: \omega \rightarrow f^{-1}[B]$. From $B \subset f[\omega]$ it follows that $f[g[\omega]]=f\left[f^{-1}[\omega]\right]=B$ and this implies that $\| \tau_{x} \cap f[g[\omega]]$ is infinite $\| \leq b$. Finally, since $\lim \sup x \circ f \circ g=$ $\| \tau_{x} \cap f[g[\omega]]$ is infinite $\|$ it follows (e).

(h) $\Leftrightarrow$ (i) It follows from the fact that $a \leq b$ iff $b^{\prime} \leq a^{\prime}$.

(i) $\Rightarrow$ (j) We have two cases

$1^{\circ} b^{\prime} \Vdash$ " $\tau_{x}$ is finite". Than for an arbitrary tall ideal $\mathcal{J} \subset P(\omega)$ we have $b^{\prime} \Vdash \forall B \in \tilde{\mathcal{J}}$ " $\tau_{x} \cap B$ is finite".

$2^{\circ} b^{\prime}$ If " $\tau_{x}$ is finite". Let

$$
\mathcal{J}=\left\{B \subset \omega: b^{\prime} \Vdash{ }^{*} \tau_{x} \cap \tilde{B} \text { is finite" }\right\}
$$

Then, $\omega \notin \mathcal{J}$ and $\emptyset \in \mathcal{J}$. Also, $\mathcal{J}$ is closed toward subsets and finite joins, which implies that $\mathcal{J}$ is an ideal. By (c), for each $A \in[\omega]^{\omega}$ there exists $B \in[A]^{\omega}$ such that $b^{\prime} \Vdash$ " $\tau_{x} \cap \check{B}$ is finite". So, $B \in \mathcal{J} \cap[\omega]^{\omega}$ such that $B \subset A$, which implies that $\mathcal{J}$ is a tall ideal. Finally, for each $B \in \mathcal{J}$ we have $b^{\prime} \Vdash$ " $\tau_{x} \cap \bar{B}$ is finite", which implies $b^{\prime} \Vdash \forall B \in \mathcal{J}^{\prime \prime} \tau_{x} \cap \check{B}$ is finite". 
(j) $\Rightarrow$ (k) If $\omega \notin \mathcal{J}$, then in the of proof (i) $\Rightarrow$ (j) part $2^{\circ}$ is proved that $\mathcal{J}$ is a tall ideal. If $\omega \in \mathcal{J}$, then, by conclusion from the same part of the proof, it follows that $\mathcal{J}=P(\omega)$.

$(\mathrm{k}) \Rightarrow$ (i) It is obvious.

Theorem 6.4.4 $\left(u=u^{2}\right)$ The following conditions are equivalent;

(a) $0 \in \lim x$;

(b) 1 It " $\tau_{x}$ is finite" or $\exists S \in[\omega]^{\omega} \exists \mathcal{J} \in \operatorname{Tall}(S) 1 \Vdash \tau_{x} \subset \bar{S} \wedge \forall I \in \overrightarrow{\mathcal{J}}\left|\tau_{x} \cap I\right|<\tilde{\omega} ;$

(c) $\exists \mathcal{J} \in \operatorname{Tall}(\omega) 1 \Vdash \forall I \in \mathcal{J}\left|\tau_{x} \cap I\right|<\tilde{\omega}$.

Proof. (a) $\Leftrightarrow$ (c) It follows from the equivalence of (a) and (j) in Theorem 6.4.3.

(b) $\Rightarrow$ (a) If $1 \Vdash \tau_{x}$ is finite, then $\lim \sup x=0 \in \lim x$. So, let $S \in[\omega]^{\omega}$ and $\mathcal{J}_{S} \subset P(S)$ be a tall ideal on the set $S$ such that

$$
1 \Vdash \tau_{x} \subset \check{S} \wedge \forall I \in \check{\mathcal{J}}_{S}\left|\tau_{x} \cap I\right|<\check{\omega} .
$$

Let us prove that

$$
\forall A \in[\omega]^{\omega} \exists g \in \omega \rightarrow A \| \check{g}^{-1}\left[\tau_{x}\right] \text { is finite } \|=1 .
$$

This is equivalent to (d) in Theorem 6.4.1, for $b=0$, since $\| \check{g}^{-1}\left[\tau_{x}\right]$ is finite $\|^{\prime}=$ $\| \check{g}^{-1}\left[\tau_{x}\right]$ is infinite $\|$.

Let $A \in[\omega]^{\omega}$. We can distinguish two cases.

$1^{\circ}|A \cap S|<\omega$. Let $g: \omega \rightarrow A$ be a bijection and let $G$ be a $\mathbb{B}$-generic filter over $V$. Since, $1 \Vdash \tau_{x} \subset S$, we have that $\left(\tau_{x}\right)_{G} \subset S$, and therefore $\left|A \cap\left(\tau_{x}\right)_{G}\right|<\omega$. From this and from the fact that $g$ is a bijection it follows

$$
\left|g^{-1}\left[\left(\tau_{x}\right)_{G}\right]\right|=\left|g^{-1}\left[\left(\tau_{x}\right)_{G} \cap A\right]\right|<\omega .
$$

Hence, $1 \Vdash \check{g}^{-1}\left[\tau_{x}\right]$ is finite.

$2^{\circ}|A \cap S|=\omega$. Since $\mathcal{J}_{S}$ is a tall ideal, there exists $I \in \mathcal{J}_{S} \cap[\omega]^{\omega}$ such that $I \subset A \cap S$. Let $g: \omega \rightarrow I$ be a bijection. Clearly, $g: \omega \rightarrow A$. Let $G$ be a $\mathbb{B}$-generic filter over $V$. From $I \Vdash$ " $\tau_{x}$ kills $\breve{\mathcal{J}}_{S}$ " it follows that $\left|I \cap\left(\tau_{x}\right)_{G}\right|<\omega$. From this and from the fact that $g$ is a bijection it follows

$$
\left|g^{-1}\left[\left(\tau_{x}\right)_{G}\right]\right|=\left|g^{-1}\left[\left(\tau_{x}\right)_{G} \cap I\right]\right|<\omega .
$$

Hence, $\left\|\left|\check{g}^{-1}\left[\tau_{x}\right]\right|<\breve{\omega}\right\|=1$.

(a) $\Rightarrow$ (b) From Theorem 6.4 .3 (b) it follows that

$$
\forall A \in[\omega]^{\omega} \exists B \in[A]^{\omega} \| \tau_{x} \cap \dot{B} \text { is finite } \|=1 .
$$


If 1 1t " $\tau_{x}$ is finite", then the proof is complete. Therefore, let us suppose that there exists $b \in \mathbb{B}$ such that

$$
b \Vdash " \tau_{x} \text { is infinite". }
$$

Let $S=\left\{n \in \omega: x_{n}>0\right\}$. Formula (6.24) implies that $S$ is infinite and that $1 \Vdash \tau_{x} \subset \tilde{S}$. Let

$$
\mathcal{J}_{S}=\left\{B \subset S: \| \tau_{x} \cap \check{B} \text { is finite } \|=1\right\} \text {. }
$$

Claim $1 . \mathcal{J}_{S}$ is a tall ideal in $P(S)$.

Proof of Claim 1. Let us firstly prove that $\mathcal{J}_{S}$ is an ideal in $P(S)$. (I1) $\emptyset \in \mathcal{J}_{S}$ obviously. Let us suppose that $S \in \mathcal{J}_{S}$, i.e. $\| \tau_{x} \cap \bar{S}$ is finite $\|=1$. From $1 \Vdash \tau_{x} \subset$ $\check{S}$ it follows that $1 \Vdash$ " $\tau_{x}$ is finite". A contradiction.

(I2) and (13) Obvious.

Let us prove that $\mathcal{J}_{S}$ is tall in $P(S)$. Let $A \in[S]^{\omega}$. By (6.23), there exists $B \subset A$ such that $\| \tau_{x} \cap \tilde{B}$ is finite $\|=1$, which implies that $B \in \mathcal{J}_{S}$, proving that $\mathcal{J}_{S}$ is tall and completing the proof of Claim 1 .

The final part of the proof follows directly from the definition of $\mathcal{J}_{S}$.

Corollary 6.4.5 $\left(u=u^{2}\right) \lim x=\bigcap_{f \in \omega \uparrow \omega} \bigcup_{g \in \omega \uparrow \omega} \lambda^{\top}(x \circ f \circ g)$.

Proof. It follows directly from Theorem 6.4.3 (a) $\Leftrightarrow$ (g).

Lemma 6.4.6 $\left(u=u^{2}\right)$ If $a, b \in \lim x$, then $a \wedge b \in \lim x$.

Proof. Let $a, b \in \lim x$. Let us prove that for all $y \prec x$ there exists $z \prec y$ such that $\lim \sup z \leq a \wedge b$. Let $y \prec x$. From $a \in \lim x$, and $y \prec x$ it follows that $a \in \lim y$. According to Lemma 6.4.1 there exists $y^{\prime} \prec y$ such that $\lim \sup y^{\prime} \leq a$. From $b \in \lim x$ and $y^{\prime} \prec x$ it follows that $b \in \lim y^{\prime}$ and, by Lemma 6.4.1, we have that there exists $z \prec y^{\prime}$ such that $\lim \sup z \leq b$. Since, limsup $z \leq \lim \sup y^{\prime} \leq a$, we obtain that limsup $z \leq a \wedge b$, which, with the fact that $z \prec y^{\prime} \prec y \prec x$, completes the proof.

Theorem 6.4.7 $\left(u=u^{2}\right)$ Let $x \in \mathbb{B}^{\omega}$. Then $\lim x=\mathbb{B}$ or $\lim x$ is countablycomplete filter in $\mathbb{B}$.

Proof. Let us suppose that $\lim x \neq \mathbb{B}$. Then

(F1) $0 \notin \lim x \ni 1$.

(F2) If $a, b \in \lim x$, from Lemma 6.4 .6 it follows that $a \wedge b \in \lim x$. 
(F3) If $a \geq b \in \lim x$ then, by Corollary $6.3 .2, a \in \lim x$.

Let us prove that $\lim x$ is countably complete. Let $b_{n} \in \lim x$, for $n \in \omega$. Then, by (F2), it follows that $c_{n}=b_{0} \wedge \ldots b_{n} \in \lim x$. Hence $c_{n} \geq c_{m}$ for $n \leq m$. The set $\lim x$ is a closed subset of $\mathbb{B}$ and, therefore, by Theorem $6.1 .2(\mathrm{c})$, $\bigwedge_{n \in \omega} c_{n}=\bigwedge_{n \in \omega} b_{n} \in \lim x$.

Theorem 6.4.8 Let $\mathbb{B}$ be a ccc weakly-distributive c.B.a. Then for each sequence $x$ there exists $b \in \mathbb{B}$ such that $\lim x=b \uparrow$.

Proof. From Theorem 6,1,15 and from that fact that $\lim x$ is a elosed set it follows that

$$
\lim x=\bigcup_{b \in \operatorname{Min}(\lim x)} b 1 .
$$

But, Lemma 6.4.6 implies that there exists only one minimal element.

So, if $\mathbb{B}$ is a weakly distributive ccc c.B.a., then $\lim x$ is a proper countably complete filter. But in general countable complete filter is not always proper. The filter of cocountable sets in $P\left(\omega_{1}\right)$ is an example of a countably complete filter which is not proper.

In the following theorem is given a characterization of Boolean algebras satisfying $u=u^{2}$ in which a priori and a posteriori limit operator are the same.

Theorem 6.4.9 $\left(u=u^{2}\right)$ The following conditions are equivalent:

(a) $\lim \neq \lambda^{\top}$;

(b) $\exists x \in \mathbb{B}^{\omega} 0 \in \lim x \backslash \lambda^{\top}(x)$;

(c) In some extension of $V$ by $\mathbb{B}$ there exists a new real;

(d) $\mathbb{B}$ is not $(\omega, 2)$ distributive.

Proof. Equivalence between (c) and (d) is well known (see [26]). Condition (b) implies (a) and implication (a) $\Rightarrow$ (b) is Lemma 6,3,7. It only remains to prove that (b) is equivalent to (c).

(b) $\Rightarrow$ (c) Let $x$ be a sequence such that $0 \in \lim x$ and $\lim \sup x=b>0$. According to Theorem 6.4 .4 , there exists a tall ideal $\mathcal{J}$ on $\omega$ such that $1 \Vdash \forall I \in$ $\mathcal{J}\left|\tau_{x} \cap I\right|<\bar{\omega}$. Let $b$ be in $G$, where $G$ is a $\mathbb{B}$-generic filter over $V$. Then, for each $I \in \mathcal{J}$ there holds $\left|\left(\tau_{x}\right)_{G} \cap I\right|<\omega$ and $\left|\left(\tau_{x}\right)_{G}\right|=\omega$. Condition $\left(\tau_{x}\right)_{G} \in V$ implies that $\mathcal{J}$ is not a tall ideal. Therefore, $\left(\tau_{x}\right)_{G}$ is a new real.

(c) $\Rightarrow$ (b) Let us suppose that $V_{\mathrm{B}}[G]$ contains a new real. According to Lemma 4,2.1, there exists a tall ideal $\mathcal{J} \subset P(\omega)$ and a new real $Y$ which kills $\mathcal{J}$. Let $\sigma=\left\{\left\langle\widetilde{n}_{n}, y_{n}\right\rangle: n \in \omega\right\}$ be a nice name such that $Y=\sigma_{G}$. So, in the extension $V_{\mathbb{B}}[G]$ we have $\left|\sigma_{G}\right|=\omega \wedge \forall I \in \mathcal{J}\left|I \cap \sigma_{G}\right|<\omega$ which implies that

$$
\||\sigma|=\omega \wedge \forall I \in \dot{\mathcal{J}}|I \cap \sigma|<\omega\|=b>0 .
$$


Let $\tau=\left\{\left\langle\vec{n}, y_{n} \wedge b\right\rangle: n \in \omega\right\}$.

Claim 1. $b \Vdash \tau=\sigma$.

Proof of Claim 1 . Let $b \in G$, where $G$ is a $\mathbb{B}$-generic filter over $V$. Then

$$
n \in \tau_{G} \Leftrightarrow x_{n} \in G \Leftrightarrow y_{n} \wedge b \in G \Leftrightarrow y_{n} \in G \Leftrightarrow n \in \sigma_{G},
$$

which proves the claim.

So,

$$
b \Vdash|\tau|=\omega \wedge \tau \text { kills } \mathcal{J} \text {. }
$$

Claim 2. $1 \Vdash \forall I \in \tilde{\mathcal{J}}|\tau \cap I|<\tilde{\omega}$.

Proof of Claim 2. If $b$ is in the generic filter $G$, from (6.5) it follows that $\forall I \in \mathcal{J}$ $\left|\tau_{G} \cap I\right|<\omega$. If $b \notin G$, then $b^{\prime} \in G$, which implies that $\tau_{G}=\emptyset$, which completes the proof of the claim.

Now, let $x_{n}=y_{n} \wedge b, n \in \omega$, and $x=\left\langle x_{n}: n \in \omega\right\rangle$. Since $\tau=\tau_{x}$, from Claim 2 and Theorem 6.4 .4 we have that $0 \in \lim x$. Since $\lim \sup x=\| \tau_{x}$ is infinite $\| \geq$ $b>0$, we have $0 \notin \lambda^{\top}(x)$.

Lemma 6.4.10 $\left(u=u^{2}\right)$ Let $x$ be a sequence in $\mathbb{B}$. Then

(a) $\lim x \subset b_{x} \uparrow$, where $b_{x}$ is defined in Definition 4.2.5;

(b) If $\lim x=b_{0} \uparrow$ then $b_{0} \geq b_{x}$.

Proof. Let us first prove (a). Let $b \in \lim x$. By Theorem 6.4.3, it is equivalent to

$$
\forall A \in[\omega]^{\omega} \exists B \in[A]^{\omega} \| \tau_{x} \cap \bar{B} \text { is infinite } \| \leq b,
$$

which implies that

$$
\bigwedge_{A \in[\omega]^{\omega}} \bigvee_{B \in[A]^{\omega}} \| \tau_{x} \cap \tilde{B} \text { is infinite } \| \leq b,
$$

which, together with Lemma 4.2.6, completes this part of the proof.

(b) follows directly from (a).

Theorem 6.4.11 $\left(u=u^{2}\right)$ Let $\mathbb{B}$ be a Boolean algebra satisfying $(\hbar)$ and let $x$ be a sequence in $\mathbb{B}$. Then $\lim x=b_{x} \uparrow$, where $b_{x}$ is as defined in Definition 4.2 .5 .

Proof. According to Theorem 6.4.7, for each sequence $x, \lim x$ is a countably complete filter. Let $b_{0}=\bigwedge \lim x$. Let us prove that $b_{0} \in \lim x$, which will imply that $\lim x=b_{0} \uparrow$.

It will be sufficient to prove condition (h) from Theorem 6.4.3, i.e.

$$
\forall A \in[\omega]^{\omega} \exists B \in\left[\left.A\right|^{\omega}\left\|\left|\tau_{x} \cap \breve{B}\right|=\check{\omega}\right\| \leq b_{0} .\right.
$$


Let $A \in[\omega]^{\omega}$. The set of $x$-stable sets is dense in $[\omega]^{\omega}$ (see Definition 5.2.3). So, there exists an $x$-stable set $B \subset A$. For each $b \in \lim x$, there exists $B_{b} \in[B]^{\omega}$ such that $\left\|\left|\tau_{x} \cap \tilde{B}_{b}\right|=\check{\omega}\right\| \leq b$. Since $B$ is $x$-stable, for each $b \in \lim x$ we have

$$
\left\|\left|\tau_{x} \cap \tilde{B}\right|=\tilde{\omega}\right\|=\left\|\left|\tau_{x} \cap \tilde{B}_{b}\right|=\tilde{\omega}\right\| \leq b .
$$

Hence, $\left\|\left|\tau_{x} \cap \dot{B}\right|=\check{\omega}\right\| \leq \bigwedge \lim x=b_{0}$, which completes the proof of (6.27).

Now, let us prove that $b_{0}=b_{x}$. From Lemma 6.4.10 we have that $b_{0} \geq b_{x}$. Let us prove that $b_{0} \leq b_{x}$, i.e.

$$
b_{0} \leq \bigvee_{A \in[\omega] \omega} \bigwedge_{B \in[A]^{\omega}}\|\| \tau_{x} \cap \check{B} \mid=\breve{\omega} \| .
$$

From $b_{0} \in \lim x$ it follows (6.27). Also we have that for each $B_{1} \subset B$ there holds $\left\|\left|\tau_{x} \cap \breve{B}_{1}\right| \leq \tilde{\omega}\right\| \leq\left\|\left|\tau_{x} \cap \tilde{B}\right|=\breve{\omega}\right\|$. Since the set of $x$-stable sets is dense, we can reformulate condition (6.27). So,

$$
\forall A \in[\omega]^{\omega} \exists B \in[A]^{\omega}\left(B \text { is } x-\text { stable } \wedge\left\|\left|\tau_{x} \cap \tilde{B}\right|=\check{\omega}\right\| \leq b_{0}\right) .
$$

Let $\mathcal{A}=\left\{A: A\right.$ is $x-$ stable $\left.\wedge\left\|\left|\tau_{x} \cap \check{A}\right|=\breve{\omega}\right\| \leq b_{0}\right\}$. Let us prove that

$$
c \stackrel{\text { def }}{=} V_{A \in \mathcal{A}}\left\|\tau_{x} \cap \check{A} \mid=\bar{\omega}\right\|=b_{0} .
$$

From (6.29) it follows that $c \leq b_{0}$. Let us suppose that $c<b_{0}$. Then $c \notin \lim x$ and therefore there exists $A_{c} \in[\omega]^{\omega t}$ such that

$$
\forall B \in\left[A_{c}\right]^{\omega}\left\|\left|\tau_{x} \cap \check{B}\right|=\check{\omega}\right\| \not c
$$

But, there exists a $x$-stable set $B_{1} \subset A_{c}$ such that $\left\|\left|\tau_{x} \cap \breve{B_{1}}\right|=\check{\omega}\right\| \leq b_{0}$, which implies that $B_{1} \in \mathcal{A}$. So,

$$
\left\|\left|\tau_{x} \cap \bar{B}_{1}\right|=\tilde{\omega}\right\| \leq \bigvee_{A \in \mathcal{A}}\left\|\left|\tau_{x} \cap \check{A}\right|=\check{\omega}\right\|=c
$$

which contradicts (6.30).

Now, let us prove that $b_{0} \leq b_{x}$, i.e.

$$
\bigvee_{A \in \mathcal{A}}\left\|\left|\tau_{x} \cap \bar{A}\right|=\tilde{\omega}\right\| \leq \bigvee_{A \in[\omega]^{\mu}} \bigwedge_{B \in[A] \omega}\left\|\left|\tau_{x} \cap \tilde{B}\right|=\dot{\omega}\right\| .
$$

First, we will prove that for each $A \in \mathcal{A}$ we have

$$
\left\|\left|\tau_{x} \cap \check{A}\right|=\tilde{\omega}\right\|=\bigwedge_{B \in[A] \omega}\left\|\left|\tau_{x} \cap \check{B}\right|=\check{\omega}\right\| .
$$

Let $A \in \mathcal{A}$. Then, $A$ is an $x$-stable set, which implies that for each $B \in[A]^{\omega}$ we have

$$
\left\|\left|\tau_{x} \cap \breve{A}\right|=\tilde{\omega}\right\|=\left\|\left|\tau_{x} \cap \breve{B}\right|=\tilde{\omega}\right\|,
$$


and therefore equality (6.32) holds, which implies that

$$
\begin{aligned}
\bigvee_{A \in \mathcal{A}}\left\|\left|\tau_{x} \cap \tilde{A}\right|=\bar{\omega}\right\| & \leq \bigvee_{A \in \mathcal{A}} \bigwedge_{B \in[A] \omega}\left\|\left|\tau_{x} \cap \tilde{B}\right|=\bar{\omega}\right\| \\
& \leq V_{A \in[\omega] \omega} \bigwedge_{B \in[A] \omega}\left\|\left|\tau_{x} \cap \tilde{B}\right|=\bar{\omega}\right\| .
\end{aligned}
$$

This proves that $b_{0} \leq b_{x}$ and completes the proof.

In the following example we calculate the a posteriori limit of some specific sequences.

Example 6.4.12 $\left(u=u^{2}\right)$ Let $\mathbb{B}$ satisfies $(\hbar)$ and $x \in \mathbb{B}^{\omega}$.

If $1 \Vdash$ " $\tau_{x}$ is finite", then $b_{x}=\lim \sup x=\| \tau_{x}$ is infinite $\|=\| \tau_{x}$ is finite $\|^{\prime}=$ $1^{\prime}=0$, which implies that $\lim x=0 \uparrow=\mathbb{B}$.

If $1 \Vdash$ " $\tau_{x}$ is supported", then $b_{x}=\limsup x=\| \tau_{x}$ is infinite $\| \geq$ $\| \tau_{x}$ is supported $\|=1$. Therefore $\lim x=\{1\}$.

If $1 \Vdash$ " $\tau_{x}$ is independent", then $b_{x}=\limsup x=\| \tau_{x}$ is infinite $\| \geq$ $\| \tau_{x}$ is independent $\|=1$. Therefore $\lim x=\{1\}$.

Theorem 6.4.13 $\left(u=u^{2}\right)$ Let $\mathbb{B}$ be a c.B.a. satisfying $(\hbar)$ and let $x$ be a sequence in $\mathbb{B}$. Let

$$
\mathcal{J}_{x} \stackrel{\text { def }}{=}\left\{I \subset \omega:\left\|\left|\tau_{x} \cap \tilde{I}\right|=\bar{\omega}\right\| \leq b_{x}\right\} .
$$

Then

(a) $\mathcal{J}_{x} \in \operatorname{Tall}(\omega) \cup\{P(\omega)\}$;

(b) $b_{x}=\limsup x \Leftrightarrow \mathcal{J}_{x}=P(\omega)$;

(c) $b_{x}^{\prime}=\left\|\forall I \in \check{\mathcal{J}}_{x}\left|\tau_{x} \cap I\right|<\bar{\omega}\right\|$;

(d) $\lim \sup x \backslash b_{x}=\| \tau_{x}$ is infinite and kills $\mathcal{J}_{x} \|$;

(e) The following conditions are equivalent:

(i) $b_{x}<\limsup x$;

(ii) $\lim x \neq \lambda^{\top}(x)$;

(iii) $\mathcal{J}_{x} \in \operatorname{Tall}(\omega)$;

(iv) There exists extension in which $\tau_{x}$ is infinite and kills a tall ideal;

(v) $\left\|\left|\tau_{x}\right|=\omega \wedge \forall I \in \check{\mathcal{I}}_{x}\left|\tau_{x} \cap I\right|<\omega\right\|>0$.

(f) The following conditions are equivalent:

(i) $b_{x}=\lim \sup x$;

(ii) $1 \Vdash \tau_{x}$ is finite or supported or independent over some $A \in[\omega]^{\omega}$.

Proof.

(a) Theorem 6.4.11 implies that $b_{x} \in \lim x$. By Fact 4.1 .2 (b) and Theorem $6.4 .3(\mathrm{k})$ it directly follows that $\mathcal{J}_{x} \in \operatorname{Tall}(\omega) \cup\{P(\omega)\}$. 
(b) We have

$\mathcal{J}_{x}=P(\omega) \Leftrightarrow \omega \in \mathcal{J}_{x} \Leftrightarrow\left\|\left|\tau_{x}\right|=\omega\right\| \leq b_{x} \Leftrightarrow \limsup x \leq b_{x} \Leftrightarrow b_{x}=\limsup x$.

(c) This is equivalent to $b_{x}=V_{I \in \mathcal{J}_{x}}\left\|\left|\tau_{x} \cap \check{I}\right|=\check{\omega}\right\|$. Since $\left\|\left|\tau_{x} \cap \check{I}\right|=\check{\omega}\right\| \leq b_{x}$ for each $I \in \mathcal{J}_{x}$, we have $V_{I \in \mathcal{J}_{x}}\left\|\left|\tau_{x} \cap \check{I}\right|=\check{\omega}\right\| \leq b_{x}$. Let $A \in[\omega]^{\omega}$. From (a) it follows that $\mathcal{J}_{x}$ is a tall ideal or $P(\omega)$. Hence, there exists $I \in \mathcal{J}_{x}$ such that $I \subset A$. Since, $I \in[A]^{\omega}$, we have

$$
\bigwedge_{B \in[A]^{\omega}}\left\|\left|\tau_{x x} \cap \bar{B}\right|=\check{\omega}\right\| \leq\left\|\left|\tau_{x} \cap \check{I}\right|=\omega\right\| \leq \bigvee_{I \in \mathcal{J}_{x}}\left\|\left|\tau_{x} \cap \check{I}\right|=\check{\omega}\right\| .
$$

So, we have

$$
b_{x}=\bigvee_{A \in[\omega]^{\omega}} \bigwedge_{B \in[A]^{\omega}}\left\|\left|\tau_{x} \cap \check{B}\right|=\tilde{\omega}\right\| \leq \bigvee_{I \in \mathcal{J}_{x}}\left\|\left|\tau_{x} \cap \check{I}\right|=\tilde{\omega}\right\|
$$

(d) It directly follows from (c).

(e) $(i) \Leftrightarrow$ (ii) It follows from the facts that $\lim x=b_{x} \uparrow, \lambda^{\top}(x)=\limsup x \uparrow$, and that $b_{x} \leq \limsup x$.

(i) $\Rightarrow$ (iii) From (b) we have that $\mathcal{J}_{x} \neq P(\omega)$, and from (a) we have that $\mathcal{J}_{x} \in \operatorname{Tall}(\omega)$.

(iii) $\Rightarrow$ (i) From (a) it follows that $\mathcal{J}_{x} \neq P(\omega)$, which is, by (b), equivalent to $b_{x} \neq \lim \sup x$. Since $b_{x} \leq \lim \sup x$, we have $b_{x}<\lim \sup x$.

(iii) $\Rightarrow$ (iv) Let $\mathcal{J}_{x}$ be a tall ideal. From the equivalence of (iii) and (i) it follows that

$$
c=\lim \sup x \wedge b_{x}^{\prime}>0 .
$$

Let $G$ be a generic filter containing $c$. Since $c \leq \limsup x=\left\|\left|\tau_{x}\right|=\bar{\omega}\right\|$, we have

$$
\left|\left(\tau_{x}\right)_{G}\right|=\omega
$$

If $I \in \mathcal{J}_{x}$, then $c \leq b_{x}^{\prime} \leq\left\|\left|\tau_{x} \cap \check{I}\right|<\varpi\right\| \in G$. So,

$$
\forall I \in \mathcal{J}_{x}\left|\left(\tau_{x}\right)_{G} \cap I\right|<\omega,
$$

which implies that $\left(\tau_{x}\right)_{G}$ kills the tall ideal $\mathcal{J}_{x}$.

(iv) $\Rightarrow$ (iii) Let $\mathcal{J}$ be a tall ideal and $G$ a $\mathbb{B}$-generic filter over $V$ such that

$$
\left.\|\left(\tau_{x}\right)_{G}|=\omega \wedge \forall I \in \mathcal{J}|\left(\tau_{x}\right)_{G} \cap I \mid<\omega\right]^{\left.V_{b} \mid G\right]} .
$$

Fact 4.1.2 (d) implies that there exists $c \in G$ such that

$$
\begin{gathered}
c \leq\left\|\left|\tau_{x}\right|=\tilde{\omega}\right\|, \\
c \Vdash \forall I \in \tilde{\mathcal{J}}\left|\tau_{x} \cap I\right|<\bar{\omega} .
\end{gathered}
$$


Theorem 6.4.3 (i) implies that $c^{\prime} \in \lim x$, which implies that

$$
b_{x} \leq c^{\prime} .
$$

Let us suppose that $\mathcal{J}_{x}$ is not a tall ideal. Then $\mathcal{J}_{x}=P(\omega)$, which implies that $\left\|\left|\tau_{x}\right|=\tilde{\omega}\right\| \leq b_{x} \leq c^{\prime}$. From (6.33) we have that $c \leq\left\|\left|\tau_{x}\right|=\bar{\omega}\right\|$, which implies that $c \leq c^{\prime}$, and therefore that $c=0$, which is impossible, since $c \in G$.

$(i) \Rightarrow(v)$ Let $c=\lim \sup x \wedge b_{x}^{\prime}>0$. Then $c \leq\left\|\left|\tau_{x}\right|=\tilde{\omega}\right\|$ and $c \leq b_{x}^{\prime}$, which implies that

$$
\forall I \in \mathcal{J}_{x} c \leq b_{x}^{\prime} \leq\left\|\left|\tau_{x} \cap \check{I}\right|<\breve{\omega}\right\| .
$$

So,

$$
c \Vdash|f| \tau_{x}\left|=\check{\omega} \wedge \forall I \in \breve{J}_{x}\right| \tau_{x} \cap I \mid<\check{\omega} .
$$

(v) $\Rightarrow$ (iii) If we suppose that $\omega \in \mathcal{J}_{x}$, then there exists $G$, a $\mathbb{B}$-generic filter over $V$, such that $\left|\left(\tau_{x}\right)_{G}\right|=\omega$ and $\left|\left(\tau_{x}\right)\right|<\omega$.

(f)

$$
\begin{aligned}
& b_{x}=\limsup x \\
& \Leftrightarrow\left\|\left|\tau_{x}\right|=\check{\omega}\right\| \leq\left\|\exists A \in\left(\left([\omega]^{\omega}\right)^{V}\right) \forall B \in\left(\left([A]^{\omega}\right)^{V}\right)\left|\tau_{x} \cap B\right|=\check{\omega}\right\| \\
& \Leftrightarrow 1 \Vdash\left|\tau_{x}\right|<\dot{\omega} \vee \exists A \in\left(\left([\omega]^{\omega}\right)^{V}\right) \forall B \in\left(\left([A]^{\omega}\right)^{V}\right)\left|\tau_{\infty} \cap B\right|=\dot{\omega} \\
& \Leftrightarrow 1 \Vdash\left|\tau_{x}\right|<\tilde{\omega} \vee\left(\left(\tau_{x} \text { is supported } \vee \tau_{x} \text { is unsupported }\right) \wedge\right. \\
& \left.\left.\exists A \in\left(\left([\omega]^{\omega}\right)^{V}\right) \forall B \in\left(\left([A]^{\omega}\right)^{V}\right)\left|\tau_{x} \cap B\right|=\tilde{\omega}\right)\right) \\
& \Leftrightarrow 1 \Vdash\left|\tau_{x}\right|<\dot{\omega} \vee\left(\tau_{x}\right. \text { is supported } \\
& \left.\wedge \exists A \in\left(\left([\omega]^{\omega}\right)^{V}\right)^{*} \forall B \in\left(\left([A]^{\omega}\right)^{V}\right)^{*}\left|\tau_{x} \cap B\right|=\breve{\omega}\right) \vee \\
& \left(\forall S \in\left([\omega]^{\omega}\right)^{V}\left|S \backslash \tau_{x}\right|=\tilde{\omega} \wedge\right. \\
& \left.\left.\exists A \in\left(\left([\omega]^{\omega}\right)^{V}\right) \forall B \in\left(\left([A]^{\omega}\right)^{V}\right)\left|\tau_{x} \cap B\right|=\check{\omega}\right)\right) \\
& \Leftrightarrow 1 \Vdash\left|\tau_{x}\right|<\dot{\omega} \vee \tau_{x} \text { is supported } \vee \\
& \left(\tau_{x x} \text { is unsupported } \wedge \exists A \in\left([\omega]^{\omega}\right)^{V} \forall B \in\left([A]^{\omega}\right)^{V}\right. \\
& \left.\left(\left|\tau_{x} \cap B\right|=\bar{\omega} \wedge\left|B \backslash \tau_{x}\right|=\bar{\omega}\right)\right)
\end{aligned}
$$

Finally, from the tautology $p \vee(\neg p \wedge r) \Leftrightarrow p \wedge r$ it follows the equivalence.

\subsection{A representation of closed sets in $\left\langle P(\omega), \mathcal{O}^{\top}\right\rangle$}

The Boolean algebra $P(\omega)$ is in the class of complete algebras the only atomic ccc algebra. Therefore, it does not add any new set by forcing. As a consequence we have that it is $(\omega, 2)$-distributive. Also we have the following. 
Theorem 6.5.1 In the topological space $\left\langle P(\omega), \mathcal{O}^{\top}\right\rangle$ we have
(a) $\lim x=(\lim \sup x) \uparrow$ for each sequence $x$;
(b) Convergence a priori equals convergence a posteriori;
(c) Operator $\lambda^{\uparrow}$ fultills condition (L3);
(d) $u^{2}=u$;
(e) $\left\langle P(\omega), \mathcal{O}^{\top}\right\rangle$ is a Fréchet space.

Proof. (a) is direct consequence of Lemmas 6.3.6. (b) and (c) follows from (a) and Lemma 6.3.7. Since $P(\omega)$ is weakly-distributive and ccc (which implies b-cc), there holds $u=u^{2}$, which implies that $\left\langle P(\omega), \mathcal{O}^{\top}\right\rangle$ is a Fréchet space.

By Theorem 6.1.15, each closed set $F$ in $\left\langle P(\omega), \mathcal{O}^{\dagger}\right\rangle$ can be represented by

$$
F=\bigcup_{b \in \operatorname{Min}(F)}(b \uparrow),
$$

where $\operatorname{Min}(F)$ is the set of minimal elements of $F$. But in $P(\omega)$ we can characterize sets $B$ such that the set $\bigcup_{b \in B}(b \uparrow)$ is closed in $\left\langle P(\omega), \mathcal{O}^{\top}\right\rangle$. For that we need the notion of subbase countably compact spaces.

\subsubsection{Subbase countably compact spaces}

Definition 6.5.2 [23] Let $\langle X, \mathcal{O}\rangle$ be a topological space,

- If $\mathcal{S}$ is a subbase for the topology $\mathcal{O}$, the space $\langle X, \mathcal{O}\rangle$ is an $\mathcal{S}$-countably compact $\left(S\right.$-CC) space iff $\forall A \in[X]^{\omega} \exists x \in X \forall S \in S(x \in S \Rightarrow|S \cap A|=\omega)$.

$+\langle X, \mathcal{O}\rangle$ is a subbase countably compact (SCC) space iff there exists a subbase $\mathcal{S}$ for $\mathcal{O}$ such that $\langle X, \mathcal{O}\rangle$ is an $\mathcal{S}$-CC space.

Theorem 6.5.3 [23, p. 227] Each Lindelöf SCC space is compact.

Definition 6.5.4 Let $X \neq \emptyset . \mathcal{S} \subset P(X)$ is a $T_{1} S C C$ subbase iff
(i) $\bigcup \mathcal{S}=X$;
(ii) $\forall x, y \in X(x \neq y \Rightarrow \exists S \in \mathcal{S}(x \in S \not \supset y))$;
(iii) $\forall A \in[X]^{\omega} \exists x \in X \forall S \in S(x \in S \Rightarrow|S \cap A|=\omega)$.

Lemma 6.5.5 Let $X \neq \emptyset$ and $\mathcal{S}=\left\{S_{k}: k \in \omega\right\} \subset P(X)$. Then the following conditions are equivalent:

(a) $\mathcal{S}$ is a $T_{1}$ SCC subbase;

(b) The topology $\mathcal{O}$ generated by $\mathcal{S}$ is $T_{1}$ compact topology on $X$.

Proof. (a) $\Rightarrow$ (b) By (ii) of Definition 6.5.4, $\langle X, \mathcal{O}\rangle$ is a $T_{1}$-space. Obviously, the weight of $\langle X, \mathcal{O}\rangle$ is $\omega$, which implies that the space is Lindelof, and by Theorem 6.5 .3 , compact 
(b) $\Rightarrow$ (a) Let $\mathcal{O}$ be the topology generated by $\mathcal{S}=\left\{S_{k}: k \in \omega\right\} \subset P(X)$. By (b), $\langle X, \mathcal{O}\rangle$ is a $T_{1}$ compact space. Let us prove three conditions from Definition 6.5.4. Condition $(i)$ is obviously fulfilled.

Let us prove condition (ii). Let $x, y \in X$ such that $x \neq y$. Since $\langle X, \mathcal{O}\rangle$ is a $T_{1}$ space, there exists a open set $U$ such that $x \in U \not \not y y$. We can w.l.o.g. suppose that $U$ belongs to the base generated by $\mathcal{S}$, i.e. $U=\bigcap_{i=1}^{n} S_{k_{i}}$. Obviously $x \in S_{k_{i}}$ for each $i \leq n$, and there exists $j \leq n$ such that $y \notin S_{k_{j}}$.

It remains to prove condition (iii). Let $A \in[X]^{\omega}$. Since $X$ is a compact space, there exists an accumulation point of the set $A$, namely $x_{0}$, i.e.

$$
\forall U \in \mathcal{U}\left(x_{0}\right)\left(U \cap A \backslash\left\{x_{0}\right\}\right) \neq \emptyset .
$$

Since $\langle X, \mathcal{O}\rangle$ is a $T_{1}$ space, one can easily verify that $|U \cap A|=\omega$ for each $U \in \mathcal{U}\left(x_{0}\right)$. Let $S \in \mathcal{S}$ such that $x_{0} \in S$. Then $S \in \mathcal{U}\left(x_{0}\right)$, and therefore $|S \cap A|=\omega$.

Theorem 6.5.6 Let $\langle X, \mathcal{O}\rangle$ be a $T_{1}$ compact second-countable space. If $\mathcal{S} \subset \mathcal{O}$ is a countable subbase for the topology $\mathcal{O}$, then $\mathcal{S}$ is a $T_{1}$ SCC subbase.

Proof. The proof is analogous to the proof of Lemma 6.5 .5 (b) $\Rightarrow$ (a).

Theorem 6.5.7 If $\langle X, \mathcal{O})$ is a compact second-countable $T_{1}$ space, then $|X| \leq \omega$ or $|X|=\mathrm{c}$.

Proof. Since $\langle X, \mathcal{O}\rangle$ is a second-countable space there holds $w(X)=\omega$. According to [16, Th. 1.5.1], $|X| \leq 2^{w(X)}=2^{\omega}=\mathrm{c}$.

According to $[16,3.12 .7]$ for $T_{1}$ space $\langle X, \mathcal{O}\rangle$ there holds $\psi(X) \leq \chi(X)$, where $\psi(X)$ is the pseudocharacter of $X$, and $\chi(X)$ is the character of $X$. Since $\langle X, \mathcal{O}\rangle$ is second-countable, it is also first-countable, which implies that $\psi(X)=$ $\omega$. By [27, p. 84], for each $T_{1}$ compact space $\langle X, \mathcal{O}\rangle$ with countable pseudocharacter we have that $|X| \leq \omega$ or $|X|=c$, which completes the proof.

Example 6.5.8 According to Theorem 6.5.7, if $X$ is $T_{1}$ compact second-countable space, then $|X| \leq \omega$ or $|X|=c$. So, regarding the size of $X$ we distinguish three classes of compact second-countable spaces.

$1^{\circ}|X|<\omega$. The only examples are finite discrete spaces $D(n), n \in \omega$.

$2^{\circ}|X|=\omega$. Some of the examples are: cofinite topology on $\omega$ and countable successor ordinals.

$3^{\circ}|X|=c$. Well known examples are $[0,1]$ and the Hilbert cube $[0,1]^{\omega}$. In fact, all such spaces can be embedded into $\mathbb{A}^{\omega}$, the Alexandroff cube. If they 
are $T_{2}$ spaces, then there exists a closed embedding into $[0,1]^{\omega}$. If they are zerodimensional then there exists a closed embedding into $2^{\omega}$.

Generally, the family of compact second-countable spaces is finitely additive, countably multiplicative and hereditary with the respect to closed subsets.

For more details about SCC spaces we refer the reader to the paper by J. Gerlits, I. Juhász and Z. Szentmiklóssy [23].

\subsubsection{Minimal elements of the closed set - $\operatorname{Min}(\mathrm{F})$}

Definition 6.5.9 For a mapping $f: X \rightarrow P(Y)$ let $f^{*}: Y \rightarrow P(X)$ be defined by

$$
f^{*}(y)=\{x \in X: y \in f(x)\}
$$

Lemma 6.5.10 If $f: X \rightarrow P(Y)$ then $f^{* *}=f$.

Proof. Since $f^{*}: Y \rightarrow P(X)$, it is obvious that $f^{* *}: X \rightarrow P(Y)$. So, $f^{* *}(x)=$ $\left\{y \in Y: x \in f^{*}(y)\right\}=\{y \in Y ; y \in f(x)\}=f(x)$.

Since $P(\omega)$ is a cec Boolean algebra, according to Theorem 6.1.15, each closed set $F$ can be represented by $F=\bigcup_{B \in \operatorname{Min}(F)} B \uparrow$. So, indexing the set of the minimal elements of the set $F$ by $B: X \rightarrow \operatorname{Min}(F)$ such that $B(x)=B_{x}$, we have $F=\bigcup_{x \in X}\left(B_{x} \uparrow\right)$. On the other hand, for an arbitrary $X$ and $B: X \rightarrow$ $P(\omega)$ the set $A=\bigcup_{x \in X}\left(B_{x} \uparrow\right)$ is not necessarily a closed set. For instance, if $\left\langle B_{n}: n \in \omega\right\rangle$ is a strictly decreasing sequence, then $A=\bigcup_{n \in \omega}\left(B_{n} \uparrow\right)$ is not a closed set in $\left\langle P(\omega), \mathcal{O}^{\dagger}\right\rangle$. Even the incomparability of the sets $B_{n}$ is not sufficient for the set $A$ to be closed, since $A=\bigcup_{n \in \omega}(\{n\} \uparrow)=P(\omega) \backslash\{\emptyset\}$ is not closed. So, the aim of this section is to characterize functions $B: X \rightarrow P(\omega)$ such that the set $\bigcup_{x \in X} B_{x} \uparrow$ is closed in $\left\langle P(\omega), \mathcal{O}^{\top}\right\rangle$.

Lemma 6.5.11 If $B: X \rightarrow P(\omega)$ is one-to-one mapping and $F=\bigcup_{x \in X}\left(B_{x} \uparrow\right)$. then the following conditions are equivalent:

(a) Elements $B_{x}, x \in X$, are incomparable;

(b) $\forall x, y \in X\left(x \neq y \Rightarrow \exists k \in \omega\left(x \in S_{k} \not \ngtr y\right)\right)$, where $S=B^{*}$;

(c) $\left\{B_{x}: x \in X\right\}=\operatorname{Min}(F)$.

Proof. (a) $\Rightarrow$ (b) Let $x, y \in X$ such that $x \neq y$. Then $B_{x} \not \subset B_{y}$, which implies that there exists $k \in \omega$ such that $k \in B_{x}$ and $k \notin B_{y}$. Therefore, $x \in S_{k} \not \supset y$.

(b) $\Rightarrow$ (a) Let $x, y \in X$ such that $x \neq y$. By (b), there exists $k \in \omega$ such that $x \in S_{k} \not \supset y$. So, $k \in B_{x}$, and $k \notin B_{y}$, which implies that $B_{x} \not \subset B_{y}$. Analogously, there exists $l \in \omega$ such that $l \in B_{y}$ and $l \notin B_{x}$, which implies that $B_{y} \not \subset B_{x}$. 
(a) $\Rightarrow$ (c) (c) Let $x \in X$. Then $B_{x} \in F$. Let us suppose that there exists $A \in F$ such that $A \varsubsetneqq B_{x}$. From $A \in F$, we have that there exists $y \in X$ such that $B_{y} \subset A$, which implies that $B_{y} \subsetneq B_{x}$, which contradicts (a).

(D) Let $M$ be a minimal element of the set $F$. So, $M \in F$, and, therefore, there exists $x \in X$ such that $B_{x} \subset M$. From the minimality of $M$ it follows that $M=B_{x}$.

(c) $\Rightarrow$ (a) Follows directly from the fact that the minimal elements are incomparable.

For a mapping $B: X \rightarrow P(\omega)$ by $\tau^{B}=\left\{\left\langle\tilde{x}, B_{x}\right\rangle: x \in X\right\}$ we denote the corresponding nice name for a subset of $X$.

Lemma 6.5.12 If $G$ is a $P(\omega)$-generic filter over $V$ and $S=B^{*}$, where $B: X \rightarrow$ $P(\omega)$ then

(a) $G=\{k\} \uparrow$ for some $k \in \omega$;

(b) $\left(\tau^{B}\right)_{G}=S_{k}$.

Proof. (a) Since the set of atoms $\{\{k\}: k \in \omega\}$ is dense in $P(\omega)$, there exists $k \in \omega$ such that $\{k\} \in G$. Since $G$ is a filter, for each set $A$ satisfying $\{k\} \subset A$, we have $A \in G$. The existence of $B \in G$ such that $k \notin B$ would imply that $\emptyset=B \cap\{k\} \in G$, which is impossible.

(b) We have that $x \in\left(\tau^{B}\right)_{G}$ is equivalent to $B_{x} \in G$. By (a), There exists $k \in w$ such that $G=\{A: k \in A\}$. So, $B_{x} \in G$ is equivalent to $k \in B_{x}$, and this is equiyalent to $x \in S_{k}$.

Lemma 6.5.13 If $B: X \rightarrow P(\omega), S=B^{*}$ and $F=\bigcup_{x \in X}\left(B_{x} \uparrow\right)$ then the following conditions are equivalent:

(a) $F$ is closed in $P(\omega)$;

(b) $u(F)=F$;

(c) $\forall f: w \rightarrow X \exists x \in X \quad B_{x} \subset \lim \sup \left\langle B_{f(x)}\right)$;

(d) $\forall f: \omega \rightarrow X$, where $f$ is finite-to-one, $\exists x \in X B_{x} \subset \limsup \left\langle B_{f(n)}\right)$ :

(e) $\forall A \in[X]^{\omega} \exists x \in X 1 \Vdash \check{x} \in \tau^{B} \Rightarrow\left|\tau^{B} \cap \bar{A}\right|=\check{\omega}$;

(f) $\forall A \in[X]^{\omega} \exists x \in X \forall k \in \omega\left(x \in S_{k} \Rightarrow\left|S_{k} \cap A\right|=\omega\right)$.

Proof. (a) $\Leftrightarrow$ (b) Follows from Theorem 2,3.13.

(b) $\Rightarrow$ (c) Let $f: u \rightarrow X$. Then $\left\langle B_{f(n)} \pm n \in \omega\right\rangle$ is a sequence in $F$. Since $u(F)=F$ we have $\limsup \left\langle B_{f(n)}\right\rangle \in F$, but then there exists $x \in X$ such that $B_{x} \subset \limsup \left\langle B_{f(n)}\right\rangle$.

(c) $\Rightarrow$ (d) Obvious. 
(d) $\Rightarrow$ (b) Let $\left\langle A_{n}: n \in \omega\right\rangle \in F^{\omega}$. For each $n \in \omega$, there exists $x_{n} \in X$ such that $B_{x_{n}} \subset A_{n}$. So, $\limsup \left\langle B_{x_{n}}\right\rangle \subset \limsup \left\langle A_{n}\right\rangle$. Since, $F$ is upward closed set, it will be sufficient to prove that $\lim \sup \left\langle B_{x_{n}}\right\rangle \in F$. Let $f: \omega \rightarrow X$ be the mapping defined by $f(n)=x_{n}$.

If $f$ is finite-to-one, then, by (d), there exists $x \in X$ such that $B_{x} \subset \lim \sup \left\langle B_{x_{n}}\right\rangle$, which implies that $\lim \sup \left\langle B_{x_{n}}\right\rangle \in F$.

If $f$ is not finite-to-one, then there exists $x \in X$ such that $x=x_{n}$ for infinitely many $n \in \omega$. Then $\lim \sup \left\langle B_{x_{n}}\right\rangle=\bigcap_{k \in \omega} \bigcup_{n \geq k} B_{x_{n}} \supset \bigcap_{k \in \omega} B_{x}=B_{x}$, which implies that $\lim \sup \left\langle B_{x_{n}}\right\rangle \in F$.

(d) $\Rightarrow$ (e) Let $A \in[X]^{\omega}$. Then there exists an injection $f: \omega \rightarrow X$ such that $f[\omega]=A$. By (d), there exists $x \in X$ such that

$$
B_{x} \subset \lim \sup \left\langle B_{f(n)}\right\rangle \text {. }
$$

We have that $B_{x}=\left\|\check{x} \in \tau^{B}\right\|$ and $\limsup \left\langle B_{f(n)}\right\rangle=\| \forall k \in \dot{\omega} \exists n \geq k \check{f}(n) \in$ $\tau^{B}\|=\|\left(f^{-1}\right)\left[\tau^{B}\right]$ is infinite $\|=\| \tau^{B} \cap(f[\omega])$ is infinite $\|=\| \tau^{B} \cap \tilde{A}$ is infinite $\|$. Formula (6.36) implies that $\left\|\tilde{x} \in \tau^{B}\right\| \leq \| \tau^{B} \cap \breve{A}$ is infinite $\|$, i.e. $1 \Vdash \check{x} \in \tau^{B} \Rightarrow$ $\left|\tau^{B} \cap \check{A}\right|=\check{\omega}$.

(e) $\Rightarrow$ (f) Let $A \in[X]^{\omega}$. By (e), there exists $x \in X$ such that

$$
\left\|\check{x} \in \tau^{B} \Rightarrow\left|\tau^{B} \cap \bar{A}\right|=\check{\omega}\right\|=1 .
$$

By Lemma 6.5.12, for each generic filter $G$ there exists $k \in \omega$ such that $G=\{k\} \uparrow$ and $\left(\tau_{B}\right)_{G}=S_{k}$. So, let $G_{k}=\{k\} \uparrow$. Then (6.37) can be rewritten by

$$
\forall k \in \omega\left[\check{x}_{G_{k}} \in \tau_{G_{k}}^{B} \Rightarrow\left|\tau_{G_{k}}^{B} \cap \check{A}_{G_{k}}\right|=\omega\right]^{\left.V \mid G_{k}\right]} .
$$

Since $V\left[G_{k}\right]=V$, by Lemma 6.5 .12 , there holds $\forall k \in \omega\left(x \in S_{k} \Rightarrow\left|S_{k} \cap A\right|=\right.$ $\omega)$.

(f) $\Rightarrow$ (d) Let $f: \omega \rightarrow X$ be a finite-to-one mapping. Then for $A=f[\omega] \in$ $[X]^{\omega}$ there exists $x \in X$ such that

$$
\forall k \in \omega\left(x \in S_{k} \Rightarrow\left|S_{k} \cap A\right|=\omega\right) .
$$

Also

$$
\left.\left|S_{k} \cap A\right|=\omega \Leftrightarrow\left|S_{k} \cap f\right| \omega\right]|=\omega \Leftrightarrow| f^{-1}\left[S_{k}\right] \mid=\omega \Leftrightarrow \forall l \in \omega \exists n \geq l n \in f^{-1}\left[S_{k}\right],
$$

Since $n \in f^{-1}\left[S_{k}\right] \Leftrightarrow f(n) \in S_{k} \Leftrightarrow k \in B_{f(n)}$, we have

$$
\begin{aligned}
\left|S_{k} \cap A\right|=\omega & \Leftrightarrow \forall l \in \omega \exists n \geq l k \in B_{f(n)} \\
& \Leftrightarrow k \in \bigcap_{l \in \omega} \bigcup_{n \geq l} B_{f(n)}=\lim \sup \left\langle B_{f(n)}\right\rangle .
\end{aligned}
$$


So, $(6.38)$ is equivalent to

$$
\forall k \in \omega\left(k \in B_{x} \Rightarrow k \in \lim \sup \left\langle B_{f(\pi i)}\right\rangle\right),
$$

which implies that $B_{I} \subset \lim \sup \left\langle B_{f(n)}\right\rangle$.

Theorem 6.5.14 If $B: X \rightarrow P(\omega), S=B^{*}$ and $F=\bigcup_{x \in X} B_{x x} \uparrow$ then the following conditions are equivalent:

(a) $F$ is a closed set different from $P(\omega)$ and $\operatorname{Min}(F)=\left\{B_{x}: x \in X\right\}$;

(b) $\mathcal{S}=\left\{S_{k}: k \in \omega\right\}$ is a $T_{1}$ SCC subbase which generates some second countable topology on $X$;

(c) $\mathcal{S}=\left\{S_{k}: k \in \omega\right\}$ is subbase for a $T_{1}$ compact second countable topology on $X$.

Proof. (a) $\Rightarrow$ (b) We will prove conditions from Definition 6.5.2.

(i) From $F \neq P(\omega)$ and $B_{x} \in F$, it follows that $B_{x} \neq \emptyset$, and, therefore, there exists $k \in B_{x}$. So, $x \in S_{k} \subset \bigcup \mathcal{S}$.

(ii) Follows directly from Lemma 6.5.11.

(iii) Follows directly from Theorem 6.5.13.

The fact that the topology is second countable follows from the countability of the subbase $\mathcal{S}$.

(b) $\Rightarrow$ (a) Firstly, let us prove that $F \neq P(\omega)$. From $\bigcup_{k \in \omega} S_{k}=X$ it follows that $\forall x \in X \exists k \in \omega x \in S_{k}$, which implies that $\forall x \in X \exists k \in \omega k \in B_{x}$. So, $\forall x \in X B_{x} \neq \emptyset$, which gives that $\emptyset \notin F$.

The minimality of $B_{x}$ follows directly from Lemma 6.5.11 and condition (ii) from Definition 6.5.2. The fact that $F$ is closed follows directly from the equivalence of conditions (a) and (f) in Theorem 6.5.13 and from condition (iii) in Definition 6.5.2.

The equivalence between (b) and (c) follows from Lemma 6.5.5.

Theorem 6.5.15 If $F=\bigcup_{B \in \operatorname{Min}(F)} B \uparrow$ is closed in $P(\omega)$ then $|\operatorname{Min}(F)| \leq \omega$ or $|\operatorname{Min}(F)|=c$.

Proof. Follows directly from Theorem 6.5.7.

Theorem 6.5.16 For a closed set $F, \operatorname{Min}(F)$ does not contain infinite antichain.

Proof. Let us suppose that in $\operatorname{Min}(F)$ there exists a countable antichain $\left\langle A_{n}: n \in\right.$ $\omega)$. Then $\emptyset=\limsup \left(A_{n}\right)$, and therefore $\emptyset \in F$. which implies that $\operatorname{Min}(F)=$ $\{0\}$. A contradiction. 
Example 6.5.17 $\operatorname{Min}(F)$ can contain finite antichains. Let $A_{0} \cup \dot{\cup} A_{1} \cup \dot{U} \ldots \dot{\cup} A_{n}=\omega$ be a partition of $\omega$, such that $A_{k} \neq \emptyset, k \leq n$. Then $\bigcup_{k \leq n} A_{k} \uparrow$, according to Lemma 6.1.7, is a closed set with the minimal elements $\left\{A_{0}, A_{1}, \ldots, A_{n}\right\}$, which is finite antichain.

\subsubsection{Examples of closed sets}

Let $\langle X, \mathcal{O}\rangle$ be a $T_{1}$ compact second countable space and $\mathcal{S}=\left\{S_{k}: k \in \omega\right\}$ a countable subbase of $\mathcal{O}$. Using mappings $S: \omega \rightarrow P(X)$ and $B: X \rightarrow P(\omega)$. where $S=B^{*}$, i.e.

$$
B_{x}=\left\{k \in \omega: x \in S_{k}\right\},
$$

according to Theorem 6.5.14, we obtain the set $F=\bigcup_{x \in X} B_{x} \uparrow$ which is closed in $\left\langle P(\omega), \mathcal{O}^{\top}\right\rangle$. Also, each closed set has such representation. The several examples of $T_{1}$ compact second countable spaces are given in Example 6.5.8. It is important to mention that in construction arbitrary countable subbase can be used.

Example 6.5.18 We will construct closed set using cofinite topology on $P(\omega)$. So, let us enumerate finite subsets of $\omega$ with

$$
[\omega]^{<\omega}=\left\{K_{k}: k \in \omega\right\} .
$$

Then a countable subbase is $\left\{S_{k}=\omega \backslash K_{k}: k \in \omega\right\}$. Therefore, $B_{n}=\{k \in \omega$ : $\left.n \notin K_{k}\right\}$ and it generates the closed set

$$
F=\bigcup_{n \in \omega}\left\{k \in \omega: n \notin K_{k}\right\} \uparrow .
$$

On the other hand, if we choose another subbase, for instance, $\left\{S_{k}=\omega \backslash\{k\}\right.$ : $k \in \omega\}$, then $B_{n}=\omega \backslash\{n\}$ and we obtained the closed set

$$
F=\bigcup_{n \in \omega}(\omega \backslash\{n\}) \uparrow=\{A \subset \omega:|\omega \backslash A| \leq 1\} .
$$

Example 6.5.19 Let $\left\{A_{n}: n \in \omega\right\} \subset P(\omega) \backslash\{\emptyset\}$ be a family of disjoint sets, and let $B_{n}=\omega \backslash A_{n}$. Let $F=\bigcup_{n \in \omega} B_{n} \uparrow$. From Lemma 6.5 .11 we conclude that $B_{n}$, $n \in \omega$ are incomparable. Let $\left\langle C_{l} \div l \in \omega\right\rangle$ be an arbitrary sequence in $F$. Then, for each $l \in \omega$ there exists $f(l)$ such that $B_{f(l)} \subset C_{l}$. If $f$ is finite-to-one then

$$
\begin{aligned}
\limsup \left\langle B_{f(l)}\right\rangle & =\bigcap_{k \in \omega} \bigcup_{l \geq k} B_{f(l)} \\
& =\bigcap_{k \in \omega} \bigcup_{l \geq k} \omega \backslash A_{f(l)} \\
& =\omega \backslash \bigcup_{k \in \omega} \bigcup_{l \geq k} A_{f(l)} \\
& =\omega \backslash \emptyset=\omega .
\end{aligned}
$$


If not, then there exists $B_{n_{0}}$ such that $f(l)=n_{0}$ for infinitely many $l \in \omega$. So,

$$
\lim \sup \left\langle B_{f(l)}\right\rangle=\bigcap_{k \in \omega} \bigcup_{l \geq k} B_{f(l)} \supset \bigcap_{k \in \omega} \bigcup_{l \geq k} B_{n_{0}}=B_{n_{0}} .
$$

Therefore, $F$ is a closed set.

The set $S_{k}=\left\{n \in \omega: k \in B_{n}\right\}=\left\{n \in \omega: k \notin A_{n}\right\}$. If $k \in \omega \backslash \bigcup_{n \in \omega} A_{n}$, then $S_{k}=\omega$, otherwise, if $k \in A_{n}$, then $S_{k}=\omega \backslash\{n\}$. So, we have obtained the same subbase as in the second part of Example 6,5.11. 
Obvious is the most dangerous word in mathematics.

E. T. Bell

\section{Chapter 7}

\section{Other topologies}

In this chapter the properties of several other a priori limit operators and topologies generated by them are investigated. Also, $\mathcal{O}^{+}$, the dual topology of $\mathcal{O}^{\top}$ is introduced. Using the join of these two topologies as a subbase of a topological space, the new topology, $\mathcal{O}^{*}$, is obtained. It is proved that the topological limits in $\mathcal{O}^{*}$ and $\tau_{s}$ are equal in some classes of Boolean algebras and that in $P(\omega)$ these two topologies are equal.

\subsection{Topologies generated by other a priori limit operators}

Let $\mathbb{B}$ be a complete Boolean algebra. Using the algebraic convergence (see Definition 3.3.12) we have obtained the sequential topology on $\mathbb{B}$ (see Chapter 5). The algebraic convergence, as an a priori limit operator, is defined using Boolean values $\| \tau_{x}$ is cofinite $\|$ and $\| \tau_{x}$ is infinite $\|$, where $\tau_{x}$ is the nice name for a subset of $\omega$ determined by the sequence $x$.

In this chapter we will investigate topologies obtained by a priori limit operators which are defined using some Boolean values given in Theorem 4.2.2.

In the sequel we will use the following notation:

$$
\begin{aligned}
& v_{0}(x)=\| \tau_{x} \text { is cofinite } \| \\
& v_{1}(x)=\| \tau_{x} \text { is old infinite } \| ; \\
& v_{2}(x)=\| \tau_{x} \text { is supported } \| ; \\
& v_{3}(x)=\| \tau_{x} \text { is infinite dependent } \| ;
\end{aligned}
$$




$$
v_{4}(x)=\| \tau_{x} \text { is infinite } \| .
$$

According to Theorem 4.2 .3 we have

$$
v_{0}(x) \leq v_{1}(x) \leq v_{2}(x) \leq v_{3}(x) \leq v_{4}(x) .
$$

Using these Boolean values, we define a priori limit operators on $\mathbb{B}$.

Definition 7.1.1 For $i \in\{0,1,2,3,4\}$ let $\lambda_{i}: \mathbb{B}^{\omega} \rightarrow P(\mathbb{B})$ be the a priori limit operator defined by

$$
\lambda_{i}(x)=\left\{\begin{array}{cc}
\left\{v_{4}(x)\right\} & \text { if } v_{i}(x)=v_{4}(x) \\
\emptyset & \text { if } v_{i}(x)<v_{4}(x)
\end{array}\right.
$$

According to (7.1), (7,2) and Theorem 2,3.20, we have the following.

\section{Corollary 7.1.2}

(a) $\lambda_{0} \leq \lambda_{1} \leq \lambda_{2} \leq \lambda_{3} \leq \lambda_{4}$;

(b) $\mathcal{O}_{4} \subset \mathcal{O}_{3} \subset \mathcal{O}_{2} \subset \mathcal{O}_{1} \subset \mathcal{O}_{0}$.

Proof. (a) follows directly from the sequence of inequalities (7.1), and (b) follows from (a) and Theorem 2.3.20.

The operator $\lambda_{0}$ coincides with the algebraic convergence. Therefore, the corresponding topology $\mathcal{O}_{0}$ is equal to the sequential topology $\tau_{s}$. The operator $\lambda_{4}$ is equal to $\lambda_{\text {sup }}$ defined in Chapter 6, which gives $\mathcal{O}_{4}=\mathcal{O}^{\dagger}$. In the sequel the properties of the topologies on a complete Boolean algebra generated by other three a priori limit operators will be examined.

First we will consider the operator $\lambda_{2}$. By Theorem 4.2.2 we have

$$
\lambda_{2}(x)=\left\{\begin{array}{cl}
\left\{\bigwedge_{k \in \omega} \bigvee_{n \geq k} x_{n}\right\} & \bigvee_{A \in[\omega] \omega} \bigwedge_{n \in A} x_{n}=\bigwedge_{k \in \omega} \bigvee_{n \geq k} x_{n i} \\
\emptyset & \bigvee_{A \in[\omega] \omega} \bigwedge_{n \in A} x_{n}<\bigwedge_{k \in \omega} \bigvee_{n \geq k} x_{n}
\end{array}\right.
$$

Theorem 7.1.3 Let $x$ be a sequence in a c.B.a. $\mathbb{B}$. Then $\lambda_{2}(x) \neq \emptyset$ iff $1 \Vdash$ " $\tau_{x}$ is finite or supported".

Proof. $(\Rightarrow)$ Let $\lambda_{2}(y) \neq \emptyset$. Then $\lambda_{2}(y)=\{b\}$, where $b=\| \tau_{x}$ is supported $\|=$ $\left\|\left|\tau_{x}\right|=\tilde{\omega}\right\|$. Let $G$ be a $\mathbb{B}$-generic filter over $V$. If $b \in G$, then $\left(\tau_{x}\right)_{G}$ is a supported set. If $b \notin G$, since $b^{\prime}=\left\|\left|\tau_{x}\right|=\tilde{\omega}\right\|^{\prime}=\left\|\left|\tau_{x}\right|<\omega\right\| \in G$, we have $\left(\tau_{x}\right)_{G}$ is finite.

Hence, in each generic extension $V_{\mathbb{B}}[G],\left(\tau_{x}\right)_{G}$ is finite or supported.

$(\Leftrightarrow)$ Let $1 \mathbb{F}$ " $\tau_{x}$ is finite or supported" and let us suppose that $\lambda_{2}(x)=\emptyset$. Then $b=\| \tau_{x}$ is supported $\|<\|\left|\tau_{x}\right|=\tilde{\omega} \|=c$. Let $p=c \wedge b^{\prime}>0$, and let $G$ be a $\mathbb{B}$-generic filter over $V$ such that $p \in G$. Since $c \in G$, we have that $\left(\tau_{x}\right)_{G}$ is 
infinite, and $b^{\prime}=\| \tau_{x}$ is not supported $\| \in G$ implies that $\left(\tau_{x}\right)_{G}$ is not supported. Therefore, in $V_{\mathbb{B}}[G],\left(\tau_{x}\right)_{G}$ is infinite and unsupported, a contradiction.

Theorem 7.1.4 The operator $\lambda_{2}$ fulfills condition (L1), but does not fulfill condition (L2).

Proof. First, let us prove that $\lambda_{2}$ fulfills (L1). Let $b$ be an arbitrary element of a c.B.a. $\mathbb{B}$. A corresponding name for the constant sequence $\langle b: n \in \omega\rangle$ is $\tau_{\langle b\rangle}=\{\langle\bar{n}, b\rangle: n \in \omega\}$. Since $\left\|\tau_{\langle b\rangle} \mid=\tilde{\omega}\right\|=\bigwedge_{k \in \omega} \bigvee_{n>k} b=\bigwedge_{k \in \omega}=b$, and $\| \tau_{\langle b\rangle}$ is supported $\|=\Lambda_{A \in[\omega] \omega} \bigvee_{n \in A} b=b$, we have $\lambda_{2}(\langle\hat{b}\rangle)=\{b\}$.

Now, let us show that it does not satisfy (L2). Let $x=\langle 0,1,0,1, \ldots\rangle$ be a sequence in $\mathbb{B}$. Since $1 \Vdash \tau_{x}=\{1,3,5, \ldots\}$, we have $\left\|\left|\tau_{x}\right|=\tilde{\omega}\right\|=\| \tau_{x}$ is supported $\|=1$. Therefore $\lambda_{2}(x)=\{1\}$. But, the sequence $y=\langle 0,0,0, \ldots\rangle$ is a subsequence of $x$ such that $\lambda_{2}(y)=\{0\} \not \supset 1$.

Therefore we will close the operator $\lambda_{2}$ under (L2).

Theorem 7.1.5 For each $x \in \mathbb{B}^{\omega}$ there holds $\bar{\lambda}_{2}(x)=(\limsup x) \uparrow=|| \tau_{x} \mid=$ $\tilde{\omega} \| \uparrow$.

Proof. The closure of operator $\lambda_{2}$ under (L2) is defined by (see Theorem 2.3.7)

$$
\bar{\lambda}_{2}(x)=\bigcup_{y \in \mathbb{B}^{\omega}, f \in \omega \top \omega, x=y \circ f} \lambda_{2}(y) .
$$

Let us prove that $\bar{\lambda}_{2}(x)=(\limsup x) \uparrow$ for each sequence $x=\left\langle x_{n}: n \in \omega\right\rangle$ in $\mathbb{B}$.

(C) Let $b \in \bar{\lambda}_{2}(x)$. Then, there exists a sequence $y$ in $\mathbb{B}$ and a function $f \in \omega^{\dagger \omega}$ such that $x=y \circ f$ and $b \in \lambda_{2}(y)$. Therefore, $b=\limsup y$ and, according to Corollary 3.3.7, limsup $x \leq \lim \sup y=b$, which implies $b \in(\lim \sup x) \uparrow$.

(つ) Let $b \geq\left\|\left|\tau_{x}\right|=\check{\omega}\right\|$. Let us define a sequence $y$ in $\mathbb{B}$ and function $f, g \in$ $\omega^{\top \omega}$ by

$$
y=\left\langle x_{0}, b, x_{1}, b, x_{2}, \ldots\right\rangle, \quad f(k)=2 k, g(k)=2 k+1 .
$$

Obviously, $x=y \circ f$, and $z=y \circ g=\langle b\rangle$. Dividing the set $\omega$ into two sets of the even and odd numbers, $P$ and $N$ respectively, we have

$$
\begin{aligned}
\left\|\left|\tau_{y}\right|=\tilde{\omega}\right\| & \left.=\|\left|\tau_{y} \cap \tilde{P}\right|=\dot{\omega} \vee \mid \tau_{y} \cap \tilde{N}\right]=\tilde{\omega} \| \\
& =\left\|\left|\tau_{y} \cap \tilde{f}[\omega]\right|=\tilde{\omega}\right\| \vee\left\|\mid \tau_{y} \cap \dot{g}[\omega]\right\|=\tilde{\omega} \| \\
& =\left\|\left|\tau_{x}\right|=\check{\omega}\right\| \vee\left\|\left|\tau_{z}\right|=\check{\omega}\right\| \\
& =\left\|\left|\tau_{x}\right|=\bar{\omega}\right\| \vee b \\
& =b .
\end{aligned}
$$


Also $b \Vdash \dot{N} \subset \tau_{y}$, which implies that $b \Vdash \tau_{y}$ is supported, so

$$
b \leq \| \tau_{y} \text { is supported }\|\leq\|\left|\tau_{y}\right|=\check{\omega} \|=b .
$$

Hence, $b \in \lambda_{2}(y)$ and since $x=y \circ f$ we have that $b \in \bar{\lambda}_{2}(x)$.

Closing the a priori limit operator $\lambda_{2}$ under (L2) we have obtained the operator $\lambda^{\uparrow}$. So, we can conclude that $\mathcal{O}_{2}=\mathcal{O}^{\dagger}$. By Corollary 7.1.2 (b) it follows that $\mathcal{O}_{3}=\mathcal{O}^{\dagger}$.

Now, let us consider the topology $\mathcal{O}_{1}$, which is generated by the operator $\lambda_{1}$. Firstly, we will will examine when the $\lambda_{1}$-limit of a sequence is not the empty set.

Theorem 7.1.6 $\lambda_{1}(x) \neq \emptyset$ iff $1 \Vdash$ " $\tau_{x}$ is an old set".

Proof.

$$
\begin{aligned}
\lambda_{1}(x) \neq \emptyset & \Leftrightarrow \| \tau_{x} \text { is infinite and old }\|=\| \tau_{x} \text { is infinite } \| \\
& \Leftrightarrow \| \tau_{x} \text { is infinite }\|\wedge\| \tau_{x} \text { is old }\|=\| \tau_{x} \text { is infinite } \| \\
& \Leftrightarrow \| \tau_{x} \text { is infinite }\|\leq\| \tau_{x} \text { is old } \| \\
& \Leftrightarrow 1 \Vdash \tau_{x} \text { is infinite } \Rightarrow \tau_{x} \text { is old } \\
& \Leftrightarrow 1 \Vdash \tau_{x} \text { is finite } \vee \tau_{x} \text { is old } \\
& \Leftrightarrow 1 \Vdash \tau_{x} \text { is old }
\end{aligned}
$$

The operator $\lambda_{1}$ satisfies condition (L1) since, for a constant sequence $x=$ $\langle b, b, \ldots$,$\rangle and each \mathbb{B}$-generic filter $G$ over $V$, we have that $\tau_{x}=\omega$ or $\tau_{x}=\emptyset$ which are old sets.

For the sequence $x=\langle 1,0,1,0, \ldots\rangle$ we have that $\mathrm{L} \Vdash \tau_{x}=\breve{P}$, where $P$ is the set of even numbers of $\omega$, so it is an old set. Therefore, by Theorem 7.1.6 and the definition of $\lambda_{1}$ we have $\lambda_{1}(x)=\{\limsup x\}=1$. The sequence $y=\langle 0,0,0, \ldots\rangle$ is a subsequence of $x$ and we have that $1 \Vdash \tau_{y}=\emptyset$, which implies that $\lambda_{1}(y)$ is not empty and, by definition, we have $\lambda_{1}(y)=0$, witnessing that $\lambda_{1}$ does not satisfy (L2).

Now we will consider the closure of $\lambda_{1}$ under (L2), denoted by $\bar{\lambda}_{1}$.

Theorem 7.1.7 $\bar{\lambda}_{1}=\bar{\lambda}_{2}$ iff $\mathbb{B}$ is $(\omega, 2)$-distributive.

Proof. $(\Rightarrow)$ Let us suppose that $\bar{\lambda}_{1}=\bar{\lambda}_{2}$. Then, by Theorem 7.1.5, for each $y \in \mathbb{B}^{\omega}$ there holds $(\lim \sup y) \uparrow=\bar{\lambda}_{2}(y)=\bar{\lambda}_{1}(y)$. Therefore

$$
\forall y \in \mathbb{B}^{\omega} \limsup y \in \bar{\lambda}_{1}(y) .
$$


Let us suppose that $\mathbb{B}$ is not an $(\omega, 2)$-distributive Boolean algebra. Then there exists an extension containing a new real. So, there exists $y \in \mathbb{B}^{\omega}$ such that $\| \tau_{y}$ is new $\|=b>0$. Since each new real is infinite we have

$$
0<b=\| \tau_{y} \text { is new }\|\leq\| \tau_{y} \text { is infinite } \| .
$$

From (7.4) and definition of $\bar{\lambda}_{1}$ it follows that there exists $x \succ y$ and $f \in \omega^{\dagger \omega}$ such that $y=x \circ f$ and limsup $y \in \lambda_{1}(x)$. So, $\lambda_{1}(x) \neq \emptyset$ and, according to Theorem 7.1 .6 ,

$$
1 \text { It } \tau_{x} \text { is old. }
$$

From $\lambda_{1}(x) \neq \emptyset$ we have that limsup $x \in \lambda_{1}(x)$. Since $\lambda_{1}(x)$ is a singleton we conclude that $\limsup y=\lim \sup x$, i.e.

$$
\| \tau_{y} \text { is infinite }\|=\| \tau_{x} \text { is infinite } \| .
$$

From (7.5) and (7.7) it follows that

$$
b \Vdash \tau_{y} \text { is new } \wedge \tau_{x} \text { is infinite } \wedge \tau_{y} \text { is infinite. }
$$

From $1 \Vdash \tau_{y}=f^{-1}\left[\tau_{x}\right]$, together with (7.6) and (7.8) we have

$$
b \text { ॥ } \tau_{y} \text { is new } \wedge \tau_{x} \text { is old } \wedge \tau_{y}=\tilde{f}^{-1}\left[\tau_{x}\right] .
$$

For a $\mathbb{B}$-generic filter $G$ over $V$ containing $b$ we have that $\left(\tau_{x}\right)_{G} \in V, f \in V$ and, therefore $\left(\tau_{y}\right)_{G}=f^{-1}\left[\left(\tau_{x}\right)_{G}\right] \in V$, contradicting the fact that $\tau_{y}$ is a new real.

$(\Leftarrow)$ Let $\mathbb{B}$ be an $(\omega, 2)$-distributive c.B.a. Then each generic filter does not add new reals, which implies that for each sequence $x$ we have $1 \Vdash \tau_{x}$ is old. So, $\lambda_{1}(x)=\{\limsup x\}=\lambda_{\text {sup }}(x)$. Therefore, from Theorems 6.1.1 and 7.1.5, we conclude that $\bar{\lambda}_{1}=\bar{\lambda}_{2}$.

Corollary 7.1.8 If $\mathbb{B}$ is $(\omega, 2)$-distributive, then $\mathcal{O}_{2}=\mathcal{O}_{1}$.

We have seen that if we close the operators $\lambda_{1}$ and $\lambda_{2}$ under (L2) we do not obtain the same operators in general. But, this is not sufficient to have two different Iopologies. In the sequel we will prove that $\mathcal{O}_{1}=\mathcal{O}_{2}$.

Lemma 7.1.9 Let $x=\left\langle x_{n}: n \in \omega\right\rangle$ be a sequence in $\mathbb{B}$ and $A \subset \mathbb{B}$. Then

(a) If $x$ is a decreasing sequence, then $\lambda_{1}(x)=\{\bigwedge x\}$

(b) $\bar{\lambda}_{1}(x)=\bar{\lambda}_{1}(x) 1$.

(c) $A \uparrow \subset u_{\bar{\lambda}_{1}}(A)$.

(d) $\mathrm{cl}_{\omega_{1}}^{1}(A)=\mathrm{cl}_{\omega_{1}}^{1}(A \uparrow)$, where $\mathrm{cl}_{\omega_{1}}^{1}$ is defined using the a priori limit operator $\bar{\lambda}_{1}$ (see Theorem 2.3.14).

(e) $\operatorname{Dec}(A \uparrow) \subset u_{\lambda_{1}}(A \uparrow) \subset u_{\bar{\lambda}_{1}}(A \uparrow)$, where Dec is defined in Definition 6.1.11. 
Proof,

(a) Since for a decreasing sequence $x=\left\langle x_{n}: n \in \omega\right\rangle$ we have limsup $x=$ $\bigwedge\left\{x_{n}: n \in \omega\right\}$, the statement in (a) follows directly from Lemma 4.2.3.

(b) Obviously there holds $\lambda_{1}(x) \subset \lambda_{1}(x) \uparrow$, for each sequence $x$. Let us prove the opposite inclusion. Let $a \in \bar{\lambda}_{1}(x)$ and let $b \geq a$. Then, there exists a sequence $y=\left\langle y_{n}: n \in \omega\right\rangle$ such that $x \prec y$ and $a=\lim \sup y$. For the sequence $z=\left\langle y_{0}, b, y_{1}, b, y_{2}, b, \ldots\right\rangle$ we have limsup $z=b$ and $x \prec z$. So, $b \in \bar{\lambda}_{1}(x)$.

(c) Let $b \in A \uparrow$. Then there exists $a \in A$ such that $a \leq b$. Since $\langle a, a, a, \ldots\rangle \in$ $A^{\omega}$ and since $a \in \bar{\lambda}_{1}(\langle a, a, \ldots\rangle)=\bar{\lambda}_{1}(\langle a, a, \ldots\rangle) \uparrow$, there holds $b \in \bar{\lambda}_{1}(\langle a, a, \ldots\rangle) \subset u_{\bar{\lambda}_{1}}(A)$.

(d) Obviously, $\mathrm{cl}_{\omega_{1}}^{1}(A) \subset \mathrm{cl}_{\omega_{1}}^{1}(A \uparrow)$. From $u_{\bar{\lambda}_{1}}(A) \subset u_{\bar{\lambda}_{1}}(A \uparrow)$ and $A \uparrow \subset$ $u_{\bar{\lambda}_{1}}(A)$ it follows that $\operatorname{cl}_{\omega}^{1}(A \uparrow)=\operatorname{cl}_{\omega}^{1}(A)$, which implies $\mathrm{cl}_{\omega_{1}}^{1}(A)=\operatorname{cl}_{\omega_{1}}^{1}(A \uparrow)$.

(e) Let $x$ be a decreasing sequence in $A \uparrow$. Let us prove that $\bigwedge x \in u_{\lambda_{1}}(A \uparrow)$. Since

$$
1 \Vdash \tau_{x}=\omega \backslash k \text { or } \tau_{x}=\dot{\emptyset},
$$

we have that 1 If $\tau_{x}$ is old, which, by (a) implies that $\bigwedge x \in u_{\lambda_{1}} x$. The second inclusion follows from the fact that $\lambda_{1} \subset \bar{\lambda}_{1}$.

Theorem 7.1.10 Let $A \subset \mathbb{B}$.

(a) $\operatorname{Dec}_{\omega_{1}}(A) \subset \operatorname{cl}_{\omega_{1}}^{1}(A)$;

(b) $\mathrm{Cl}_{\omega_{1}}^{1}(A) \subset \mathrm{cl}_{\omega_{1}}^{2}(A)$;

(c) $\mathrm{cl}_{\omega_{1}}^{2}(A)=\operatorname{Dec}_{\omega_{1}}(A)$;

(d) $\mathrm{cl}_{\omega_{1}}^{1}(A)=\mathrm{cl}_{\omega_{1}}^{2}(A)$.

Proof. (a) From Lemma 7.1 .9 (e) it follows that $\operatorname{Dec}_{1}(A)=\operatorname{Dec}(A \uparrow) \subset u_{\bar{\lambda}_{1}}(A \uparrow$ ) $\subset \mathrm{cl}_{\omega_{1}}^{1}(A \uparrow)$. Let us suppose that for each $\alpha<\gamma$ holds

$$
\operatorname{Dec}_{\alpha}(A) \subset \operatorname{cl}_{\omega_{1}}^{1}(A \uparrow) \text {. }
$$

If $\gamma=\beta+1$ then $\operatorname{Dec}_{\beta}(A) \subset \mathrm{cl}_{\omega_{1}}^{1}(A \uparrow)$ which implies that $\operatorname{Dec}_{\gamma}(A)=$ $\operatorname{Dec}\left(\operatorname{Dec}_{\beta}(A)\right) \subset \operatorname{Dec}\left(\mathrm{cl}_{\omega_{1}}^{1}(A \uparrow)\right)=\mathrm{cl}_{\omega_{1}}^{1}(A \uparrow)$.

If $\gamma$ is a limit ordinal, then $\operatorname{Dec} \gamma(A)=\bigcup_{\alpha<\gamma} \operatorname{Dec}_{\alpha}(A) \subset \mathrm{cl}_{\omega_{1}}^{1}(A \uparrow)$.

So, $\operatorname{Dec}_{\omega_{1}}(A) \subset \operatorname{cl}_{\omega_{1}}^{1}(A \uparrow)$, and by Lemma 7.1.9 (d), we obtain that $\operatorname{Dec}_{\omega_{1}}(A)$ $\subset \mathrm{cl}_{\omega_{1}}^{1}(A)$.

(b) This inclusion follows directly from the fact that $\mathcal{O}_{1} \subset \mathcal{O}_{2}$.

(c) This is Theorem 6.1.14.

(d) The equality follows from (a), (b) and (c).

Corollary 7.1.11 $\mathcal{O}_{2}=\mathcal{O}_{1}$.

Proof. This equality follows directly from Theorem 7.1.10 (d). 


\subsection{The topology $\mathcal{O}^{1}$}

In Chapter 6 the topological space $\left\langle\mathbb{B}, \mathcal{O}^{\dagger}\right\rangle$ generated by the a priori limit operator $\lambda_{\text {sup }}$, defined by $\lambda_{\text {sup }}(x)=\{\limsup x\}$, is investigated. Analogously, we define the a priori operator $\lambda_{\text {inf }}: \mathbb{B}^{\omega} \rightarrow P(\mathbb{B})$ by

$$
\lambda_{\text {inf }}(x)=\{\lim \inf x\}\left(=\bigvee_{k \in \omega} \bigwedge_{n \geq k} x_{n}=\| \tau_{x} \text { is cofinite } \|\right) .
$$

As in Theorem 6.1.1, we obtain that the closure of $\lambda_{\text {inf }}$ under (L2) is $\bar{\lambda}_{\text {inf }}(x)=$ $(\lim \inf x) \downarrow$. Therefore, instead of $\bar{\lambda}_{i n}$, we will use the notation $\lambda \downarrow$, and the topology generated by $\lambda^{\perp}$ will be denoted by $\mathcal{O}^{\perp}$. Let

$$
u_{\lambda \downarrow}(A)=\bigcup_{y \in A^{\omega}} \lambda^{\downarrow}(y)=\bigcup_{y \in A^{\omega}}(\lim \inf y) \downarrow .
$$

Since the operator $\lambda^{\downarrow}$ fulfills conditions (L1) and (L2), by Theorem 2.3.13, a set $A$ is closed in the space $\left\langle\mathbb{B}, \mathcal{O}^{\downarrow}\right\rangle$ iff $u_{\lambda^{L}}(A)=A$.

Topologies $\mathcal{O}^{\dagger}$ and $\mathcal{O}^{\dagger}$ are defined by two dual a priori limit operators. Therefore, we can expect some similarities among them. Let us denote $A^{\prime}=\left\{a^{\prime}: a \in\right.$ $A\}$ and $y^{\prime}=\left\langle y_{n}^{\prime}: n \in \omega\right\rangle$.

Theorem 7.2.1 The mapping $f:\left\langle\mathbb{B}, O^{\dagger}\right\rangle \rightarrow\left\langle\mathbb{B}, O^{\uparrow}\right\rangle$ defined by $f(a)=a^{\prime}$ is a homeomorphism.

Proof. This mapping is obviously a bijection. Let us prove that this a closed mapping. Let $A$ be a subset of $\mathbb{B}$ such that $u_{\lambda \downarrow}(A)=A$. Let us prove that $f[A]$ is closed in the space $\left\langle\mathbb{B}, \mathcal{O}^{\dagger}\right\rangle$.

Obviously, $f[A]=\left\{a^{\prime}: a \in A\right\}$. Let $y$ be a sequence in $f[A]$. Then $y^{\prime}$ is a sequence in $A$. It will be sufficient to prove that $\limsup y \in f[A]$. Since, $\limsup y=\left(\liminf y^{\prime}\right)^{\prime}$ and $y^{\prime}$ is a sequence in $A$, we have that $\liminf y^{\prime} \in A$, which implies that $\limsup y=\left(\liminf y^{\prime}\right)^{\prime} \in A^{\prime}=f[A]$.

Since $f^{-1}=f$, the proof of continuity is analogous.

In the sequential topology $\tau_{s}$, for any two elements $a$ and $b$, the mapping $g(x)=(x \triangle b) \triangle a$ is an automorphism satisfying $g(a)=b$ and $g(b)=a$. For $a=1$ and $b=0$ we have that $g(x)=x^{\prime}$. So, the mapping $f$ from Theorem 7.2.1 is an automorphism of the space $\left\langle\mathbb{B}, \tau_{s}\right\rangle$. Since $O^{\top} \subset \tau_{s}$, as a consequence we obtain

Corollary 7.2.2 $O^{\downarrow} \subset \tau_{s}$.

From Theorem 7.2.1 we can analogously prove, replacing meets with joins, increasing sequences with decreasing, the same properties about topology $\mathrm{O}^{+}$as for the topology $\mathcal{O}^{\dagger}$. For instance, open sets in the space $\left\langle\mathbb{B}, \mathcal{O}^{\dagger}\right\rangle$ are upward closed, closed sets are downward closed and closed to supremums of increasing sequences, etc. 


\subsection{The topology $\mathcal{O}^{\star}$ and the connection with $\tau_{s}$}

Clearly, the set $\mathcal{P}^{\star}=\mathcal{O}^{\dagger} \cup \mathcal{O}^{-}$is a subbase of some topology on $\mathbb{B}$. Let us denote it by $\mathcal{O}^{\star}$. The base $\mathcal{B}^{\star}$ generated by the subbase $\mathcal{P}^{\star}$ contains all sets of the form $O_{1} \cap O_{2}$, where $O_{1} \in O^{\dagger}$ and $O_{2} \in O^{\perp}$. So,

$$
\mathcal{B}^{\star}=\left\{O_{1} \cap O_{2}: O_{1} \in O^{\dagger}, O_{2} \in O^{\perp}\right\}
$$

is a base for the topology $\mathcal{O}^{*}$.

Since $\mathcal{O}^{i}, \mathcal{O}^{\downarrow} \subset \tau_{s}$, we have the following.

\section{Theorem 7.3.1}

(a) $\mathcal{O}^{\star} \subset \tau_{s}$.

(b) $\lim _{\tau_{s}} \leq \lim _{\mathcal{O} *}$.

The topological limit in $\mathcal{O}^{\star}$ can be described using topological limits of $\mathcal{O}^{\dagger}$ and $\mathcal{O}^{1}$.

Theorem 7.3.2 For each sequence $y \in \mathbb{B}^{\omega}$ we have

$$
\lim _{\mathcal{O}} \cdot y=\lim _{\mathcal{O} T}(y) \cap \lim _{\mathcal{O} !}(y)+
$$

Proof.

$$
\begin{aligned}
a \in \lim _{\mathcal{O} \cdot x} \Leftrightarrow & \forall O \in \mathcal{O}^{\star}\left(a \in O \Rightarrow \exists n_{0} \in \omega \forall n \geq n_{0} x_{n} \in O\right) \\
\Leftrightarrow & \forall O \in \mathcal{B}^{\star}\left(a \in O \Rightarrow \exists n_{0} \in \omega \forall n \geq n_{0} x_{n} \in O\right) \\
\Leftrightarrow & \forall O_{1} \in \mathcal{O}^{\dagger} \forall O_{2} \in \mathcal{O}^{\downarrow} \\
& \left(a \in O_{1} \cap O_{2} \Rightarrow \exists n_{0} \in \omega \forall n \geq n_{0} x_{n} \in O_{1} \cap O_{2}\right) \\
\Leftrightarrow & \forall O_{1} \in \mathcal{O}^{\dagger}\left(a \in O_{1} \Rightarrow \exists n_{0} \in \omega \forall n \geq n_{0} x_{n} \in O_{1}\right) \\
& \wedge \forall O_{2} \in \mathcal{O}^{\downarrow}\left(a \in O_{2} \Rightarrow \exists n_{0} \in \omega \forall n \geq n_{0} x_{n} \in O_{2}\right) \\
\Leftrightarrow & a \in \lim _{\mathcal{O}^{\dagger}} x \wedge a \in \lim _{\mathcal{O}^{+}} x \\
\Leftrightarrow & a \in \lim _{\mathcal{O}^{+}} x \cap \lim _{\mathcal{O}^{\prime}} x
\end{aligned}
$$

Theorem 7.3.3 If a c.B.a. $\mathbb{B}$ satisfies condition $(\hbar)$, then $\lim _{\tau_{s}}=\lim _{\mathcal{O}^{*}}$.

Proof. Let $x \in \mathbb{B}^{\omega}$ and $a \in \lim _{\mathcal{O}} x$, Then $a \in \lim _{\mathcal{O}} x \cap \lim _{\mathcal{O} T} x$. Let $y$ be an arbitrary subsequence of $x$. Then, by Theorem 6.3.10, there exists $z^{0} \prec y$ such 
that $\lim \sup z^{0} \leq a$. Also, by the dual version of the same theorems, there exists $z \prec z_{0}$ such that liminf $z \geq a$. Now, we have

$$
\limsup z \leq \lim \sup z_{0} \leq a \leq \liminf z \text {. }
$$

Since, $\liminf z \leq \lim \sup z$, we have

$$
\forall y \prec x \exists z \prec y \limsup z=\liminf z=a,
$$

which is, by Theorem 5.1.1, equivalent to $a \in \lim _{\tau_{s}} x$. This and Theorem 7.3 .1 (b) complete the proof.

Theorem 7.3.4 If $\mathbb{B}$ is a weakly distributive b-cc c.B.a., then $\lim _{\tau_{s}}=\lim _{\mathcal{O}} \cdot$.

Proof. Let $x \in \mathbb{B}^{\omega}$ and $a \in \lim _{\mathcal{O}} \cdot x$. Then $a \in \lim _{\mathcal{O}} x \cap \lim _{\mathcal{O}} x$. By Theorem 6.4.1, there exists $z^{0} \prec x$ such that limsup $z^{0} \leq a$. The remaining of the proof is analogous to the proof of Theorem 7.3.3.

In two previous theorems we have seen that in some Boolean algebras both topologies generate the same topological limit. But this is not a sufficient condition for the equality of these two topologies. For instance, on the real line, the diserete and co-countable topology have the same topological limit (only almost-constant sequences converges).

In the sequel we will isolate the class of Boolean algebras on which these two topologies are not equal.

Theorem 7.3.5 For eacil c.B.a. $\mathbb{B}$ we have $\mathcal{N}_{0}^{d}=\mathcal{O}^{\dagger}$, where $\mathcal{N}_{0}^{d}$ is the family of downward closed neighborhoods at 0 in the sequential topology $\tau_{s}$ (see Section 5.1).

Proof. Since $\mathcal{O}^{\dagger} \subset \tau_{s}$ and since for all $O \in \mathcal{O}^{\dagger}$ there holds $O=O \downarrow$, we have that O) $\subset \mathcal{N}_{0}^{d}$.

Let $U \in \mathcal{N}_{0}^{d}$. Then $F=\mathbb{B} \backslash U$ is a upward closed set. Let us prove that $F$ is a closed set in the space $\left\langle\mathbb{B} \mathcal{O}^{\top}\right\rangle$, i.e. for each decreasing sequence $\left\langle a_{n} ; n \in \omega\right\rangle \in F^{\omega}$ there holds $a=\bigwedge_{\text {newi }} a_{n} \in F$. Since, $\lim _{\tau_{s}}\left\langle a_{n}\right\rangle=\bigwedge_{n \in \omega} a_{n}$ and since $F$ is sequentially closed in $\tau_{s}$, we have $a \in F$, which, by Theorem 6.1 .2 (c), implies that $F$ is closed in the space $\left\langle\mathbb{B}, \mathcal{O}^{\dagger}\right\rangle$.

Lemma 7.3.6 In topological space $\left\langle\mathbb{B}, \mathcal{O}^{\star}\right\rangle$ the family $\mathcal{O}^{\top}$ is a neighborhood base at 0 . 
Proof. The family

$$
\mathcal{B}_{0}^{\star}=\left\{U_{1} \cap U_{2}: U_{1} \in \mathcal{O}^{\dagger}, U_{2} \in \mathcal{O}^{\perp} \text { and } 0 \in U_{1} \cap U_{2}\right\}
$$

is a neighborhood base at 0 . For $U_{1} \cap U_{2} \in \mathcal{B}_{0}^{\star}$ we have $0 \in U_{1} \cap U_{2}$, which implies that $0 \in U_{2}$, which is equivalent to $U_{2}=\mathbb{B}$. Therefore, $U_{1} \cap U_{2}=U_{1}$, which implies that $\mathcal{B}_{0}^{\star}=\mathcal{O}^{\dagger}$.

Corollary 7.3.7 If in a topological space $\left\langle\mathbb{B}, \tau_{s}\right\rangle$ the family $\mathcal{N}_{0}^{d}$ is not a neighborhood base at 0 , then $\tau_{s} \neq \mathcal{O}^{\star}$.

Proof. Let $U \in \mathcal{N}_{0}$ such that

$$
\forall V \in \mathcal{N}_{0}^{d} V \not \subset U
$$

Let us suppose that $U \in \mathcal{O}^{\star}$. Then, by Lemma 7.3.6, there exists $U_{1} \in \mathcal{O}^{\top}$ such that $V_{1} \subset U$. The fact that $V_{1} \in \mathcal{N}_{0}^{d}$ contradicts (7.10).

Finally, we will compare these two topologies on the Boolean algebra $P(\omega)$.

Theorem 7.3.8 The topological space $\left\langle P(\kappa), \mathcal{O}^{*}\right\rangle$ is a Hausdorff space for each cardinal $\kappa$.

Proof. Let $A, B \subset \kappa$ and let us suppose, without loss of generality, that $A \backslash B \neq \emptyset$. Then there exists $\alpha \in A \backslash B$. The set $\alpha \uparrow$ is closed in the space $\left\langle\mathbb{B}, \mathcal{O}^{\dagger}\right\rangle$ and the set $(\kappa \backslash\{\alpha\}) \downarrow$ is closed in the space $\left\langle\mathbb{B}, \mathcal{O}^{\downarrow}\right.$. Therefore, both sets are closed in $\left\langle P(\kappa), \mathcal{O}^{\star}\right\rangle$, disjoint, the first one contains $A$, the second one contains $B$ and, evidently, its join is $P(\kappa)$ (each set either or either not contains $\alpha$ ).

Let us remind that $\left\langle P(\omega), \tau_{s}\right\rangle$ is a compact space (see Theorem 5.1.8). Since, in the class of Hausdorff topologies defined on the same set $X$, the compact one is the minimal one, we conclude

Theorem 7.3.9 On the Boolean algebra $P(\omega)$ we have $\tau_{s}=\mathcal{O}^{*}$. 


\section{Questions}

\section{Question 1: Is the a posteriori limit always a filter?}

In the topological space $\left\langle\mathbb{B}, \mathcal{O}^{\top}\right\rangle$, where $\mathbf{B}$ is a weakly-distributive $b$-cc c. B.a, according to Theorem 6.4 .7 , the a posteriori limit of a sequence is a $\sigma$-complete filter or $\mathbb{B}$. Further more, by Theorem 6.4 .8 , if $\mathbb{B}$ is weakly-distributive cec c.B.a, then the a posteriori limit of a sequence is a proper filter or $\mathbb{B}$.

Is the a posteriori limit of the sequence always a filter or $\mathbb{B}$ ?

\section{Question 2: The equality $\tau_{s}=\mathcal{O}^{\star}$ ?}

In section 7.3 we have analyzed properties of the topology $\mathcal{O}^{*}$, which is generated by the subbase $\mathcal{O}^{\uparrow} \cup \mathcal{O}^{\downarrow}$. It has been shown that $\mathcal{O}^{*} \subset \tau_{s}$, where $\tau_{s}$ is the sequential topology. Also, for weakly-distributive b-cc Boolean algebras, or Boolean algebras satisfying condition $(\hbar)$ the topological limits are equal. In Theorem 7.3.9 we have obtained that $\left\langle P(\omega), \tau_{s}\right\rangle=\left\langle P(\omega), \mathcal{O}^{\star}\right\rangle$.

Is the Boolean algebra $P(\omega)$ the only one satisfying the equality $\tau_{s}=\mathcal{O}^{\star}$ ? 


\section{Bibliography}

[1] U. Abraham, S. Todorčević, Partition properties of $\omega_{1}$ compatible with $\mathrm{CH}$, Fund. Math. 152 (2) (1997) 165-181.

[2] P. Antosik, On a topology of convergence, Colloguium Math. 21 (1970) 205209.

[3] B. Balcar, F. Franěk, J. Hruška, Exhaustive zero-convergence structures on Boolean algebras, Acta Univ. Carol. Math. Phys. 40 (1999) 27-41.

[4] B. Balcar, J. Pelant, P. Simon, The space of ultrafilters on N covered by nowhere dense sets, Fund. Math. 110 (1980) 11-24.

[5] B. Balcar, W. Glówczyński, T. Jech, The sequential topology on complete Boolean algebras, Fund. Math. 155 (1998) 59-78.

[6] B, Balcar, T. Jech, T. Pazák, Complete ccc Boolean algebras, the order sequential topology and a problem of von Neumann, Bull. London Math. Soc. 37 (6) (2005) 885-898.

[7] B. Balcar, T. Jech, Weak distributivity, a problem of von Neumann and the mistery of measurability, Bull. of Symb. Logic, Vol. 12 No. 2 (2006) 241266.

[8] T. Bartoszyński, H. Judah, Set Theory, On the Structure of the Real Line, A. K. Peters, 1995.

[9] G. Birkhoff, Moore-Smith convergence in general topology, Ann. of Math. 38 (1937) 39-56.

[10] G. Birkhoff, Lattice Theory, AMS Colloquium Publications 25, 1940.

[11] G. Birkhoff, Lattice Theory, Providence, RI, 1967. 
[12] A. Blass, S. Shelah, There may be simple $P_{\aleph_{1}}$ - and $P_{\mathrm{K}_{2}}$-points and the RudinKeisler ordering may be downward directed, Ann. Pure Appl. Logic 33 (3) (1987) 213-243.

[13] J. Brendle, S. Yatabe, Forcing indestructibility of MAD families, Ann. Pure Appl. Logic 132 (2-3) (2005) 271-312.

[14] P.J. Cohen, The independence of the continuum hypothesis, I, II, Proc. Nat. Acad. Sci. U.S.A. 50 (1963), 1143-I148; ibid. 51 (1964), 105-110.

[15] E.K. van Douwen, The integers and topology, in: K. Kunen and J.E. Vaughan eds., Handbook of Set-theoretic Topology (North-Holland, Amsterdam, 1984. 111-167.

[16] R. Engelking, General Topology, P.W.N. Warszawa, 1985.

[17] I. Farah, J. Zapletal, Between Maharam's and von Neumann's problems, Math. Res. Lett. 11 (5-6) (2004) 673-684.

[18] C. Ferens, A. Kamin̂ski, C. Kliss, Some examples of topological and nontopological convergence, Proceedings of the Conference on Convergence (Szczyrk, 1979), pp. 17-23, Polsk. Akad. Nauk, OddziałKatowicach, Katowice, 1980.

[19] S. P. Franklin, Spaces in which sequences suffice, Fund. Math. 57 (1965) 107-115,

[20] S. P. Franklin, Spaces in which sequences suffice II, Fund. Math. 61 (1967) 51-56.

[21] M. Fréchet, Sur quelques points du calcul foncionnel, Rend. del Circ. Mat di Palermo 22 (1906) 1-74. (in French)

[22] M, Fréchet, Sur la notion de voisinage dans les ensembles abstraits, Bull. Sci. Math. 42 (1918) 138-156. (in French)

[23] J. Gerlits, I. Juhász, Z. Szentmiklóssy, Subbase countable compactness, Studia Sci. Math. Hungarica 38 (2001) 225-231.

[24] K. Gödel, The Consistency of the Continuum Hypothesis, Annals of Mathematics Studies, no. 3., Princeton University Press, Princeton, N. J., 1940, 66 pp.

[25] P.R. Halmos, Lectures on Boolean Algebras, D. Van Nostrand Company. INC. Princeton. New Jersey, 1963. 
[26] T. Jech, Set Theory, 2. corr. ed., Springer, Berlin, 1997.

[27] I. Juhász, Cardinal functions II, in: K. Kunen and J.E. Vaughan eds., Handbook of Set-theoretic Topology (North-Holland, Amsterdam, 1984) 63-109.

[28] N.J. Kalton, J.W. Roberts, Uniformly exhaustive submeasures and nearly additive set functions, Transactions of the Amer. Math. Soc., Vol 278 (1983) 803-816.

[29] A. Kamiński, On Antosik's theorem concerning topological convergence, Proceedings of the Conference on Convergence (Szczyrk, 1979), pp. 46-49, Polsk. Akad. Nauk, OddziałKatowicach, Katowice, 1980.

[30] A. Kamiński, On characterization of topological convergence, Proceedings of the Conference on Convergence (Szczyrk, 1979), pp. 50-70, Polsk. Akad. Nauk, OddziałKatowicach, Katowice, 1980.

[31] A. Kamiński, On multivalued topological convergences, Bull. Acad. Polon. Sci. Sér. Sci. Math. 29 no. 11-12 (1981) 605-608.

[32] J. Kisyński, Convergence du type $\mathcal{L}$, Colloq. Math. 7 (1959/1960) 205-211. (in French)

[33] K. Kunen, Random and Cohen reals, in: K. Kunen and J.E. Vaughan eds., Handbook of Set-theoretic Topology, North-Holland, Amsterdam, 1984, 887911.

[34] Đ. Kurepa, Ensembles ordonnés et ramifiés, Publ. Math. Univ. Belgrade 4 (1935), 1-138.

[35] M.S. Kurilić, Independence of Boolean algebras and forcing, Ann. Pure Appl. Logic 124 (2003) 179-191.

[36] M.S. Kurilić, A. Pavlović, A posteriori convergence in complete Boolean algebras with the sequential topology, Ann. Pure Appl. Logic 148 no. 1-3 (2007) 49-62.

[37] D. Maharam, An algebraic characterization of measure algebras, Ann. of Math. 48 (1947) 154-167.

[38] R.D. Mauldin (ed.), The Scottish Book (Mathematics from the Scottish Café), Birkhäuser, Boston MA, 1981 .

[39] D. Monk, R. Bonnet (ed.), Handbook of Boolean algebras, North-Holland Publishing Co., Amsterdam, 1989. 
[40] E.H. Moore, Definition of limit in General integral analysis, Proc. Nac. Acad, Sci. (1915) 628-632.

[41] E.H. Moore, L.H. Smith, A general theory of limits, Amer. J. Math. 4 (1922) $102-121$.

[42] K. Namba, Independece proof of $\left(\omega, \omega_{1}\right)$-WDL from $(\omega, \omega)$-WDL, Comment. Math. Univ. St. Pauli 21 (1979) 47-53.

[43] F.P. Ramsey, On a problem of formal logic, Procedings of the London Mathematical Society, (2) vol. 30 (1930), 264-286.

[44] G.E. Sacks, Forcing with perfect closed sets. in: Axiomatic Set Theory, Proc. Sympos. Pure Math., Vol. XIII, Part I, Univ. California, Los Angeles, Calif., 1967, pp. 331-355. Amer. Math. Soc., Providence, R.I., 1971.

[45] S. Shelah, On cardinal invariants of the continuum, in: Axiomatic Set Theory, J.E. Baumgartner, D.A. Martin, S. Shelah (eds.), Contemporary Mathematics 31, AMS, Providence RI, 1984, 183-207.

[46] R. Sikorski, Boolean algebras, Springer-Verlag, second edition, Berlin, 1964.

[47] M. Talagrand, Maharam's problem, C.R. Acad. Sci. Paris Ser. I 342 (2006) 501-503.

[48] M. Talagrand, Maharam's problem, arXiv:math.FA/0601689v1.

[49] S. Todorčević, A dichotomy for P-ideals of countable sets, Fund. Math. 166 (3) (2000) 251-267.

[50] S. Todorčević, A problem of von Neumann and Maharam about algebras supporting continuous submeasures, Fund. Math. 183 (2) (2004) 169-183.

[51] V. Trnková, Non F-topologie, PhD Thesis, Prague, 1961.

[52] P.S. Uryson, Sur les classes (L) de M. Fréchet, Ens. Math. 25 (1926) 77-83.

[53] J.E. Vaughan, Small uncountable cardinals and topology, in: J, van Mill and G.M. Reed, eds., Open Problems in Topology, North-Holland, Amsterdam, 1990, 195-218.

[54] B. Veličković, cec forcing and splitting reals, Israel J. Math. 147 (2005) 209220 . 


\section{Index}

$A^{\omega}, 3$

$B^{A}, 3$

$[A]^{<\kappa}, 3$

$[A]^{\kappa}, 3$

$[\omega]^{\omega}, 3$

$[\omega]^{<\omega}, 3$

$\Delta_{x}, 53$

$\bar{\lambda}, 19$

$\bar{\lambda}_{\text {sup }}, 68$

$\hbar, 52$

$\lambda^{\prime}, 19$

$\lambda^{\prime}, 68$

$\lambda_{A}, 49$

$\lambda_{\text {sip }}, 67$

$\leq *, 5$

$\omega, 3$

$\omega^{\omega}$-bounding, 43

$\omega^{\dagger \omega}, 3$

$\bar{A}, 8$

$C^{*}, 5$

$\tau_{G}, 36$

$\tau_{\mathrm{s}}, 50$

$\tau_{x}, 37$

$\hat{\lambda}, 64$

$f[X], 3$

$f^{-1}[Y], 3$

$i d_{A}, 3$

$\lambda^{*}, 20$

a, 5

a posteriori convergence, 15,57 a posteriori limit operator, 15 a priori convergence, 57

a priori limit operator, 14

accumulation point, 9

adf, 5

Alexandroff cube, 101

algebraic convergence, 31,49

almost disjoint, 5

antichain, 4

$a_{x}, 39$

$\mathcal{B}^{*}, 116$

b, 5

base, 8

Boolean algebra, iii, 27

$(\kappa, \infty)$-distributive, 40

$(\kappa, \lambda)$-distributive, 40

$(\omega, \kappa)$-weakly distributive, 41

$\kappa$-cc, 27

$\kappa$-complete, 28

$\sigma$-algebra, 28

antichain, 27

atom, 27

atomic, 27

chain, 27

complete, 28

completely-distributive, 40

countable chain condition, see cce

lim sup-stable, 52, 65

partition, 27

Boolean value, 36

Boolean valued model, 35

bounded family, 5 
$b_{x}, 39$

c, 5

$c(X), 11$

Cantor cube, 10

Cantor set, 10

ccc, 4,27

cellularity, 11

$\mathrm{CH}, 66$

chain, 4

character, 11

$\chi(X), 11$

$\chi(x, X), 11$

$\mathrm{cl}_{\omega_{1}}, 23$

clopen set, 8

closed mapping, 9

closed set, 7

closed subspace, 9

closure operator, 8

coarser topology, 8

Cohen forcing, 46

compact space, 10

comparable, 4

compatible, 4

connected space, 10

continuous mapping, 9

Control Measure Problem, iii convergence, 11

countable chain condition, 4

countably compact space, 10

o, 6

$d(X), 11$

$D_{b}^{c}, 57$

Dec, 73

$\operatorname{Dec}_{\alpha}, 74$

dense set, 4, 9, 28

density, 11

derived set, 9

disconnected space, 10 dominating family, 5

downward closed set, 4

$D_{x}, 52$

embedding, 9

$f_{a}^{v}, 70$

$f_{a}^{\wedge}, 70$

filter, 28

dual, 28

generic, 36

finer topology, 8

first-countable space, 8

forcing, 35

forcing relation, 36

Fréchet, 13

Fréchet space, 13

$F_{\sigma}$-set, 8

$G_{\delta}$-set, 8

generic extension, 35

h, 5

Hausdorff space, 9

Hilbert cube, 101

homeomorphism, 9

ideal, 28

dual, 28

tall, 28

killing, 37

incompatible, 4

induced topology, 9

infimum, 4

isolated point, 8

isomorphism, 4

$J_{b}^{x}, 57$

John von Neumann, iii

$\mathcal{L}^{*}$-space, 13

$l(X), 11$ 
(L1), 13

(L2), 13

(L,3), 13

(L4), 14

$\lim x, 11$

$\lim _{0} x, 11$

liminf, 30

$\liminf \operatorname{ix}_{x \in A}, 31$

limit, 11

limit operator, 12

limit point, 11

limsup, 30

limsup $\operatorname{suA}_{x \in A}, 31$

Lindelöf number, 11

Lindelöf space, 10

linearly ordered set, 4

lower bound, 4

madf, 5

Maharam

algebra, iii

Dorothy, iii

submeasure, iii

matrix

decreasing, 29

increasing, 29

maximum, 4

measure, 32

$\sigma$-additive measure, 32

measure algebra, 33

metric, 33

Min, 74

minimum, 4

model, 35

$\mathcal{N}_{0}, 51$

$\mathcal{N}_{0}^{d}, 51$

name, 35

nice, 37

neighborhood, 8 neighborhood base, 8

normal space, 10

$\mathcal{O}^{*}, 116$

$\mathcal{O}_{\lambda, 14-16}$

open mapping, 9

open set, 4,7

open subspace, 9

$\mathcal{O}^{\top}, 68$

$\mathcal{P}^{\star}, 116$

p. 5

$P(A), 3$

$P(\kappa), 51$

$P(\omega), 66$

partial ordering, 4

partially ordered set, 4

perfect space, 8

pseudocharacter, 11

pseudointersection, 5

$\chi(X), 11$

$\psi(x, X), 11$

Random forcing, 46

real, 37

cofinal, 38

dependent, 37,38

independent, 37, 64

infinite, 38

new, 37

old infinite, 38

supported, 37, 38

unsupported, 37

regular space, 10

regular-open algebra, 46

relation, 3

antisymmetric, 4

equivalence, 4

reflexive, 3

symmetric, 4

transitive, 4 
$\mathcal{S}$-CC space, 100

$\mathcal{S}$-countably compact space, 100

$\mathcal{S}^{*}$-space, 14

5,5

Sacks forcing, 46

SCC space, 100

second-countable space, 8

separable space, 9

sequence, 11

decreasing, 29

increasing, 29

lim inf-stable, 52

limsup-stable, 52

sequences, 3

sequential space, 13

sequential topology, 50

sequentially compact space, 10, 64

set theory, 35

sfip, 5

small cardinals, 5

splitting, 5

splitting family, 5

subbase, 8

subbase countably compact space, 100

submeasure, 32

continuous, 32

exhaustive, 32

normed, 33

strictly positive, 32

uniformly exhaustive, 32

subsequence, 11

subspace, 9

supremum, 4

Suslin

algebra, iv

forcing notion, 66

Suslin algebra, 51

Suslin number, 11

Ł,5
$T_{0}$-space, 9

$T_{1}$-space, 9

$T_{2}$-space, 9

$T_{3}$-space, 10

$T_{4}$-space, 10

$\mathcal{T}_{\lambda}, 15$

topological space, 7

topology, 7

tower, 5

tree, 4

Tychonoff product, 10

$u, 69$

$u_{\lambda}, 22$

$u_{\lambda \top}, 68$

ultrafilter, 28

upper bound, 4

upward closed set, 4

Urysohn, 13

$V, 35$

$V_{\mathrm{B}}[G], 36$

$V^{\mathrm{B}}, 35$

$w(X), 11$

weakly distributive, 41

weight, 11

well-ordered set, 4

$x$-stable set, 52

zero-dimensional space, 10 


\section{Prošireni apstrakt}

\section{Nizovi u topološkim prostorima}

U proizvoljnom topološkom prostoru $\langle X, \mathcal{O}\rangle$ konvergencija nizova je preslikavanje koje nizu $x=\left\langle x_{n}: n \in \omega\right\rangle$ dodeljuje skup granica limo $x \subset X$. Generalno, proizvoljno preslikavanje $\lambda: X^{\omega} \rightarrow P(X)$ ne predstavlja topološku konvergenciju. U slučaju kada za svaki niz $x$ važi $|\lambda(x)| \leq 1, \lambda$ je topološka konvergencija akko ispunjava sledeća tri uslova:

(L1) $\forall a \in X a \in \lambda(\langle a\rangle)$,

(L2) $\forall x \in X^{\omega} \forall y \prec x \lambda(x) \subset \lambda(y)$,

(L3) $\forall x \in X^{\omega}(\forall y \prec x \exists z \prec y a \in \lambda(z) \Rightarrow a \in \lambda(x))$.

gde $y \prec x$ označava da je $y$ podniz niza $x$.

Svaka topološka konvergencija zadovoljava ova tri uslova.

Za proizvoljno preslikavanje $\lambda: X^{\omega} \rightarrow P(X)$, koga nazivamo a priori limit operator, postoji maksimalna topologija $\mathcal{O}_{\lambda}$ na $X$ takva da topološka konvergencija, koja se naziva a posteriori limit operator, sadrži operator $\lambda$.

Operator $u_{\lambda}: P(X) \rightarrow P(X)$, definisan sa $u_{\lambda}(A)=\{a \in X ; \exists x \in$ $\left.A^{\omega} a \in \lambda(x)\right\}$ dodaje skupu $A$ sve $\lambda$-granice. Ako $\lambda$ zadovoljava uslove (L1) i (L2), familiju zatvorenih skupova čine skupovi zatvoreni u odnosu na operator $\lambda$, tj. za koje važi $u_{\lambda}(A)=A$. Operator adherencije se dobija iteriranjem $u_{\lambda} \omega_{1}$ put. Prostor $\left\langle X, \mathcal{O}_{\lambda}\right)$ je sekvencijalan.

Minimalnim zatvaranjem operatora $\lambda$ da zadovoljava uslove (L1), (L2), odnosno (L3) dobijaju se novi a priori operatori koji generišu istu topologiju.

Osnovni cilj ovog rada je ispitivanje nekoliko topologija na kompletnim Bulovim algebrama koje su generisane pomoću a priori operatora.

\section{Topologija $\tau_{s}$}

Koristeći elemente kompletne Bulove algebre $\mathbb{B}$ definisane sa

$$
\limsup x=\bigwedge_{k \in \omega} \bigvee_{n \geq k} x_{n} \text { i } \quad \liminf x=\bigvee_{k \in \omega} \bigwedge_{n \geq k} x_{n},
$$


gde je $x=\left\langle x_{n}: n \in \omega\right\rangle$ niz u $\mathbb{B}$, definišemo a priori limit operator $\lambda_{\Lambda}$ sa

$$
\lambda_{A}(x)=\left\{\begin{array}{cc}
\{\limsup x\} & \text { ako je } \liminf x=\limsup x \\
\emptyset & \text { ako je } \liminf x<\lim \sup x
\end{array}\right.
$$

Ovaj operator je poznat i pod nazivom algebraska konvergencija.

S obzirom da nizu $x$ korespondira lepo ime podskupa $\omega, \tau_{x}=\left\{\left\langle\tilde{n}, x_{n}\right\rangle: n \in\right.$ $\omega\}$, važi limsup $x=\| \tau_{x}$ je beskonačan $\|\mathrm{i} \lim \inf x=\| \tau_{x}$ je kokonačan $\|$. Tako imamo da je $\lambda_{A}(x) \neq \emptyset$ akko 1 it " $\tau_{x}$ je konačan ili kokonačan". Operator $\lambda_{A}$ zadovoljava uslove (L1) i (L2), ali ne zadovoljava uslov (L3). Koristiće ga kao a priori limit operator, dobijamo topologiju poznatu kao sekvencijalna topologija na kompletnoj Bulovoj algebri koju označavamo sa $\tau_{s}$. Prostor $\left\langle\mathbb{B}, \tau_{s}\right\rangle$ je Frešeov akko je $\mathbb{B}$ slabo-distributivna i $b$-cc. To je $T_{1}$ prostor, i mada u njemu svaki niz ima najviše jednu granicu, ne mora biti Hauzdorfov. Važi da je $\left\langle B, \tau_{s}\right\rangle$ Hauzdorfov akko je metrizabilan. Sa druge starne, ako je $\mathbb{B}$ algebra mere sa merom $\mu$, tada je $d(a, b)=\mu(a \Delta b)$ metrika na $\mathbb{B}$ i topologija generisana metrikom $d$ je baš $\tau_{s}$.

Ovo je u direktnoj vezi sa nedovano rešenim fon Nojman-Maharam problemom koji u jeziku sekvencijalne topologije glasi: Da li metrizabilnost prostora $\left\langle\mathbb{B}, \tau_{s}\right\rangle$ povlači da je $\mathbb{B}$ algebra mere? Odgovor je ne. (Talagrand 2006.)

Posmatrane su osobine a posteriori konvergencije u prostoru $\left\langle\mathbb{B}, \tau_{s}\right\rangle$. Važi da niz $x$ a posteriori konvergira akko $\forall y \prec x \exists z \prec y \lim \inf z=\lim \sup z$ akko postoji tall ideal $\mathcal{J}$ za koji važi $1 \Vdash$ " $\tau_{x}$ je konačan ili $\tau_{x}$ je kokonačan ili $\tau_{x}$ ubija $\mathcal{J}$ ili $\tau_{x}^{c}$ ubija $\check{J}^{\prime \prime}$. Pokazano je da se a priori i a posteriori konvergencija poklapaju akko je algebra $(\omega, 2)$-distributivna. Izdvojena je osobina kompletnih Buovih algebri $(h)$ i za nju važi $t-c c \Rightarrow(\hbar) \Rightarrow \mathfrak{s}-c c$.

Uočena su dva elementa $a_{x} \mathrm{i} b_{x}$, gde je

$$
a_{x}=\bigwedge_{A \in[\omega]^{\omega}} \bigvee_{B \in \mid A]^{\omega}} \bigwedge_{n \in B} x_{n}, \quad b_{x}=\bigvee_{A \in[\omega]^{\omega}} \bigwedge_{B \in \mid \Lambda]^{\omega}} \bigvee_{n \in B} x_{n}
$$

Pokazano je da ako niz $x$ a posteriori konvergira tački $a$ onda je $a_{x}=b_{x}=a$. Primerom je pokazano da postoji Bulova algebra $\mathbb{B}$ i niz $x$ u $\mathbb{B}$ za koje ne važi obratna implikacija. Međutim u klasi Bulovih algebri sa uslovom $(\hbar)$ ta dva uslova su ekvivalentna.

Na kraju dela o sekvencijalnoj topologiji je pokazano da je prostor $\left\langle\mathbb{B}, \tau_{s}\right\rangle$ sekvencijalno kompaktan akko Bulova algebra zadovoljava uslov $(\hbar)$ i ako forsing pomoći nje ne dodaje nezavisni (independent) realni broj. U slučaju kada je $\mathfrak{s}=\omega_{1}$ pokazano je da je $P(\omega)$ jedina Bulova algebra u klasi Suslin forcing notions za koje je prostor sa topologijom $\tau_{s}$ sekvencijalno kompaktan. 


\section{Topologija $\mathcal{O}^{\dagger}$}

Neka je $\lambda_{\text {sup }}(x)=\{\limsup x\}$ a priori limit operator definisan na kompletnoj Bulovoj algebri. On zadovoljava osobinu (L1), ali ne i (L2), Zatvaranjem u odnosu na (L2) dobija se operator koji nizu $x$ dodeljuje skup (limsup $x) \uparrow=\{a \in \mathbb{B}: a \geq$ limsup $x\}$. Novodobijeni operator ćemo označavati sa $\lambda^{\dagger}$, a topologiju generisanu njime sa $\mathcal{O}$ !.

Prostor $\left\langle\mathbb{B}, \mathcal{O}^{\top}\right\rangle$ je povezan, kompaktan, $T_{0}$ i nikada nije $T_{1}$. Adherencija skupa $A$ se dobija, uobičajeno, iteriranje operatora $u_{\lambda r} \omega_{1}$ put. Pokazano je da se adherecija skupa može dobiti iteriranjem jednostavnijeg operatora $D e c$, koje skupu dodeljuje infimume opadajućih nizova, počevši od skupa $A \uparrow=\bigcup_{a \in A} a \uparrow$. Stoga, familiju zatvorenih skupova čine svi skupovi zatvoreni ka gore i u odnosu na infimume opadajućih nizova.

Kako je svaki zatvoren skup $F$ ujedno zatvoren ka gore, može se zapisati u obliku $F=\bigcup_{a \in F} a \uparrow$. Ako je $\mathbb{B}$ ccc kompletna Bulova algebra, onda se zatvorne skup $F$ može izraziti pomoći skupa svojih minimalnih elemenata $\operatorname{Min}(F)$ sa $F=$ $\bigcup_{a \in \operatorname{Min}(F)} a \uparrow$. Za proizvoljan skup oblika $F=\bigcup_{x \in X} q_{x} \uparrow$ dati su potrebni i dovoljni uslovi da bi skup $\left\{q_{x}: x \in X\right\}$ bio skup minimalnih elemenata skupa $F$, kao forsing karakterizacija zatvorenih skupova tog oblika, kao i zatvorenih skupova generalno.

Ako u prostoru $\left\langle\mathbb{B}, \mathcal{O}^{\top}\right\rangle$ važi jednakost $u_{\lambda \uparrow}=u_{\lambda T}^{2}$, onda je prostor Frešeov (obratno ne mora da važi) $\mathrm{i}$ adherencija skupa $A$ je $u_{\lambda \uparrow}(A)$. Pokazano je da važi $u_{\lambda T}=u_{\lambda T}^{2}$ akko je $\mathbb{B}$ b-cc i slabo-distributivna.

Posebo su ispitivane osobine a posteriori limita. Nadeni su a posteriori limiti nekih specifičnih nizova. Pokazano je da ako niz $x$ topološki konvergira tački $a$ onda za svaki podniz $y$ postoji podniz z da je $\lim \sup z \leq a$. U klasi Bulovih algebri sa uslovom $(\hbar)$ važi i obratna implikacija. Takođe, u istoj klasi, činjenica da je $\left\langle\mathbb{B}, \mathcal{O}^{\top}\right\rangle$ Frešeov prostor implicira jednakost $u_{\lambda^{\top}}=u_{\lambda \lambda^{\top}}^{2}$.

A posteriori limit u klasi kompletnih Bulovih algebri za koje važi jednakost $u_{\lambda \tau}=u_{\lambda \dagger}^{2}$ je posebno ispitan. Jezikom forsinga, kao $\mathrm{i}$ algebarskim izrazima, dato je viš̃e karakterizacija a posteriori limita i pokazano je da je u tom slučaju to prebrojivo-kompletan filter. Ako $\mathbb{B}$ zadovoljava $\mathrm{i}$ uslov $(\hbar)$, onda je a posteriori limit niza $x$ skup $b_{x} \uparrow$. Jednakost a priori i a posteriori konvergencije postoji samo ako je $\mathbb{B}(\omega, 2)$-distributivna, tj. ako forsing pomoću $\mathbb{B}$ ne dodaje novi realan broj.

Posebno je izdvojena algebra $P(\omega)$. Kod nje se a priori i a posteriori konvergencija poklapaju i važi jednakost $u_{\lambda \uparrow}=u_{\lambda \dagger}^{2}$. Kako je $P(\omega)$ ccc algebra, zatvoreni skupove zapisujemo pomoću svojih minimalnih elemenata, tj. $F=\bigcup_{b \in \operatorname{Min}(F)} b \uparrow$. Međutim, u ovoj Bulovoj algebri možemo za skup oblika $F=\bigcup_{b \in B} b \uparrow$ dati karakterizaciju skupa $B$ da bi $F$ bio zatyoren. Za preslikavanje $f: X \rightarrow P(Y)$ neka je $f^{*}: Y \rightarrow P(X)$ preslikavanje definisano sa $f^{*}(y)=\{x \in X: y \in f(x)\}$. 
Neka je $B: X \rightarrow P(\omega), S=B^{*}$ i $F=\bigcup_{x \in X} B_{x} \uparrow$. Tada je skup $F$ zatvoren sa minimalnim elementima $\operatorname{Min}(F)=\left\{B_{x}: x \in X\right\}$ akko $\mathcal{S}=\left\{S_{k}: k \in \omega\right\}$ je podbaza neke $T_{1}$ kompaktne topologije na $X$ koja zadovoljava drugu aksiomu prebrojivosti.

\section{Ostale topologije}

Sekvencijalne topologija $\tau_{s}$ je generisana pomoći operatora $\lambda_{A}$. Umesto liminf $x$ stavljajući druge Bulove vrednosti, dobijaju se novi a priori operatori. Ispitano je nekoliko takvih, i mada su im zatvaranja ka (L2) različita, svi generišu istu topologiju $\mathcal{O}^{\top}$.

Umesto a priori operatora $\lambda_{\text {sup }}$ koji nizu dodeljuje $\{\lim \sup x\}$, posmatran je njegov dual koji nizu dodeljuje $\{\lim \inf x\}$. Njegovo zatvaranje da zadovoljava (L2) je $\{\lim$ inf $x\} \downarrow$, pa se topologija koju generiše obeležava sa $\mathcal{O}+$. Preslikavanje koje elementu dodeljuje njegov komplement je homeomorfizam između prostora $\left\langle\mathbb{B}, \mathcal{O}^{\top}\right\rangle$ i $\left\langle\mathbb{B}, \mathcal{O}^{l}\right\rangle$.

Unija ove dve topologije čini podbazu neke topologije na $\mathbb{B}$. Tu topologiju ćemo označavati sa $\mathcal{O}^{*}$. Kako $\mathcal{O}^{\dagger}, \mathcal{O}^{\dagger} \subset \tau_{s}$, važi $\mathcal{O}^{\star} \subset \tau_{s}$. Pokazano je da ako za $\mathbb{B}$ važi $(\hbar)$ ili je b-cc i slabo distributivna, ondfa je $\lim _{\tau_{s}}=\lim _{\mathcal{O}}$. Međutim, jednakost limita nije dovoljna za jednakost topologija. Uočeni su neki slučajevi kada se $\mathcal{O}^{*} \mathrm{i} \tau_{s}$ ne poklapaju i pokazano je da su iste na $P(\omega)$. 


\section{Biografija}

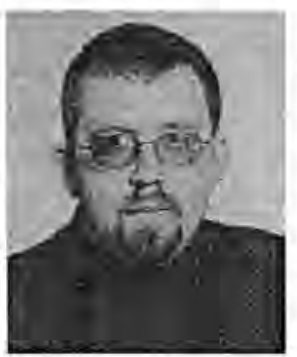

Rođen sam 3. oktobra 1974. godine u Vrbasu. Osnovnu i srednju školu, smer Matetatičko programerski saradnik, sam završio u Novom Sadu. Prorodnomatematički fakultet u Novom Sad, smer Diplomirani matematičar, sam upisao 1993. godine, kada odlazim na odsluženje vojnog roka. Studije završavam 1998. godine sa prosečnom ocenom 9,71 .

Od oktobra 1998. sam zaposlen na Prirodno-matematičkom fakultetu, Departman za matematiku i informatiku, u zvanju asistenta pripravnika do 2003, a nakon toga u zvanju asistenta. Držao sam i držim vežbe iz sledećih predmeta: Kombinatorika i teorija grafova, Obične diferencijalne jednačine, Kompleksna analiza, Terija nepokretne tačke, Algebarska topologija, Metode nepokretne tačke u ekonomiji, Poslovna matematika (za studente turizmologije).

Post-diplomske studijae sam upisao 1998. godine iz oblasti Topologija i teorija skupova. Sve ispite sam položio sa ocenom 10, a magistarsku tezu pod naslovom Topologije indukovane linearnim uređenjima odbranio sam 8. oktobra 2002, godine na PMF-u u Novom Sadu i time stekao zvanje magistra matematičkih nauka. Za magistarsku tezu sam dobio nagradu Mileva Marić-Ajnštajn, kao najbolja magistarska teza na Univerzitetu u Novom Sadu iz matematike i ínformatike 2002. godine.

Oblast mog interesovanja je skup-teoretska topologija. Do sada imam objavljena 4 naučna rada, koje sam predstavio na nekoliko međunarodnih konferencija. Od 2002. godina sam član projekta Forsing, skup-leoretska topologija i teorija modela finansiranog od strane ministarstva Republike Srbije zaduženog za nauku.

Novi Sad, septembar 2008.

mr Aleksandar Pavlović 
ㄴ. 


\section{UNIVERSITY OF NOVI SAD \\ FACULTY OF SCIENCE \\ KEY WORDS DOCUMENTATION}

\section{Accession number:}

ANO

Identification number:

INO

Document type: Monograph type

DT

Type of record: Printed text

TR

Contents code: $\mathrm{PhD}$ dissertation

CC

Author: Aleksandar Pavlović, MSc

AU

Mentor: Prof. Miloš Kurilić, $\mathrm{PhD}$

MN

Title: Sequential Topologies on Boolean Algebras

TI

Language of text: English

LT

Language of abstract: English/Serbian

LA

Country of publication: Serbia

CP

Locality of publication: Vojvodina

LP

Publication year: 2008.

PY

Publisher: Author's reprint

PU 
Publication place: Novi Sad, Faculty of Science, Dositeja Obradovića 4

PP

Physical description: $7 / x+133 / 54 / 0 / 1 / 0 / 0$

(chapters/pages/literature/tables/pictures/graphics/appendices)

PD

Scientific field: Mathematics

SF

Scientific discipline: Set-theoretic topology

SD

Subject / Key words: Boolean algebras, sequential spaces, Freshét spaces, sequential topology

\section{SKW}

\section{UC:}

Holding data: Library of the Department of Mathematics and Informatics, Novi Sad

HD

Note:

$\mathrm{N}$

Abstract: A priori limit operator $\lambda$ maps sequence of a set $X$ into a subset of $X$. There exists maximal topology on $X$ such that for each sequence $x$ there holds $\lambda(x) \subset \lim x$. The space obtained in such way is always sequential.

If a priori limit operator each sequence $x$ which satisfy $\lim \sup x=\lim \inf x$ maps into $\{\lim \sup x\}$, then we obtain the sequential topology $\tau_{s}$. If a priori limit operator maps each sequence $x$ into $\{\lim \sup x$ \}, we obtain topology denoted by $O^{\uparrow}$. Properties of these topologies, in general, on class of Boolean algebras with condition $(\hbar)$ and on class of weakly-distributive $b$-cc algebras are investigated. Also, the relations between these classes and other classes of Boolean algebras are considered.

AB

Accepted by Scientific Board on: October 6, 2006

ASB

Defended:

DE

Thesis defend board:

President: Milan Grulović, PhD, Full Professor, Faculty of Science, University of Novi Sad 
Mentor: Miloš Kurilić, PhD, Full Professor, Faculty of Science, University of Novi Sad

Member: Academic Stevan Pilipović, PhD, Full professor, Faculty of Science, University of Novi Sad

Member: Žarko Mijajlović, PhD, Full Professor, Faculty of Mathematics, University of Belgrade

DB 


\section{UNIVERZITET U NOVOM SADU \\ PRIRODNO-MATEMATIČKI FAKULTET \\ KLJUČNA DOKUMENTACIJSKA INFORMACIJA}

\section{Redni broj:}

RBR

Identifikacioni broj:

IBR

Tip dokumentacije: Monografska dokumentacija

TD

Tip zapisa: Tekstualni štampani materijal

TZ

Vrsta rada: Doktorska disertacija

VR

Autor: mr Aleksandar Pavlović

AU

Mentor: Prof, dr Miloš Kurilić

MN

Naslov rada: Sekvencijalne topologije na Bulovim algebrama NR

Jezik publikacije: engleski

JP

Jezik izvoda: engleski/srpski

JI

Zemlja publikovanja: Srbija

ZP

Uže geografsko područje: Vojvodina

UGP

Godina: 2008.

GO

Izdavač: Autorski reprint

IZ 
Mesto i adresa: Novi Sad, Prirodno-matematički fakultet, Trg Dositeja Obradovića 4

\section{MA}

Fizički opis rada: $7 / x+133 / 54 / 1 / 0 / 0 / 0$

(broj poglavlja/strana/lit. citata/tabela/slika/grafika/priloga)

FO

Naučna oblast: Matematika

NO

Naučna disciplina: Skup-teoretska topologija

ND

Predmetna odrednica/KIjučne reči: Bulove algebre, sekvencijalni prostori, Frešeovi prostori, sekvencijalna topologija.

PO

\section{UDK:}

Cuva se: u biblioteci Departmana za matematiku i informatiku, Novi Sad $\check{\mathbf{C U}}$

\section{Važna napomena:}

VN

Izvod: A priori limit operator $\lambda$ svakom nizu elemenata skupa $X$ dodeljuje neki podskup skupa $\mathrm{X}$. Tada na skupu $X$ postoji maksimalna topologija takva da za svaki niz $x$ važi $\lambda(x) \subset \lim x$. Tako dobijen prostor je uvek sekvencijalan.

Ako a priori limit operator svakom nizu $x$ koji zadovoljava uslov $\lim \sup x=$ $\lim \inf x$ dodeljuje skup $\{l i m \sup x\}$ onda se, na gore opisan način, dobija tzv. sekvencijalna topologija $\tau_{s}$. Ako a priori limit operator svakom nizu $x$ dodeljuje $\{\lim \sup x\}$, dobija se topologija označena sa $O^{\dagger}$. Ispitivane su osobine ovih topologija, generalno, na klasi Bulovih algebri koje zadovoljavaju uslov $(\hbar)$ i na klasi slabo-distributivnih i b-cc algebri, kao i odnosi ovih klasa prema drugim klasama Bulovih algebri.

17

Datum prihvatanja teme od strane NN Veća: 06. 10. 2006.

DP

Datum odbrane:

DO

Članovi komisije:

Predsednik: dr Milan Grulović, redovni profesor, Prirodno-matematički fakultet, 
Univerzitet u Novom Sadu

Mentor: dr Miloš Kurilić, redovni profesor, Prirodno-matematički fakultet, Univerzitet u Novom Sadu

Član: Akademik dr Stevan Pilipović, redovni profesor, Prirodno-matematički fakultet, Univerzitet u Novom Sadu

Član: dr Žarko Mijajlović, redovni profesor, Matematički fakultet, Beogradski Univerzitet

KO 
